Portland State University

PDXScholar

3-13-2019

\title{
Seismic Behavior of Screen Grid Core Insulated Concrete Form Walls
}

Anwer Sabah Mohammed Mohammed

Portland State University

Follow this and additional works at: https://pdxscholar.library.pdx.edu/open_access_etds

Part of the Civil Engineering Commons, and the Structural Engineering Commons Let us know how access to this document benefits you.

\section{Recommended Citation}

Mohammed, Anwer Sabah Mohammed, "Seismic Behavior of Screen Grid Core Insulated Concrete Form Walls" (2019). Dissertations and Theses. Paper 4810.

https://doi.org/10.15760/etd.6694

This Dissertation is brought to you for free and open access. It has been accepted for inclusion in Dissertations and Theses by an authorized administrator of PDXScholar. Please contact us if we can make this document more accessible: pdxscholar@pdx.edu. 
Seismic Behavior of Screen Grid Core Insulated Concrete Form Walls

\title{
by
}

Anwer Sabah Mohammed Mohammed

A dissertation submitted in partial fulfillment of the requirements for the degree of

Doctor of Philosophy

in

Civil and Environmental Engineering

\author{
Dissertation Committee: \\ Peter Dusicka, Chair \\ Franz Rad \\ Thomas Schumacher \\ Hormoz Zaerh
}

Portland State University

2019 
(C) 2019 Anwer Sabah Mohammed Mohammed 


\begin{abstract}
The use of the insulated concrete form (ICF) walls in residential buildings has increased over the past few decades. Much research has been conducted to evaluate the lateral strength of these walls by applying monotonic and cyclic loadings. In the current study, full-scale shake table experiments were employed to evaluate the in-plane behavior of four screen grid insulated concrete form (SGICF) walls. The first two wall specimens utilized dry fit insulated form blocks made from recycled expanded polystyrene granules that were bonded together with cement. When stacked, the cavities in the blocks formed a grid of cores that are evenly spaced vertically and horizontally. The walls consisted of four circular vertical cores and five circular horizontal cores reinforced with a single rebar placed nominally in the center of each core. The rest of the wall specimens were newly suggested pattern of SGICF walls, which consisted of three circular vertical cores and five circular horizontal cores. Two rebars were placed in each vertical core, and a single rebar was placed nominally in the center of each horizontal core.

Each wall was built on top of a foundation block that was designed such that failure would occur within the walls themselves. For each type of SGICF walls, steel fiber-reinforced concrete was used in one of the walls to evaluate the effect on failure modes, drift capacity, and shear strength. The results showed that the use of fiberreinforced concrete in the cores of the SGICF walls that were built out of ICF blocks had not significantly improved the drift capacity, and had exhibited lower strengths than the wall with conventional concrete. These outcomes were mainly attributed to voids in the cores that had resulted despite similarities in workability of the concrete mixes and in
\end{abstract}


techniques used to place the concrete in the walls. On the other hand, the use of the steel fiber-reinforced concrete in the cores of the newly suggested pattern of SGICF wall and the two rebars in the vertical cores had improved the drift ductility of the wall by about $63 \%$.

The evaluation of the steel fiber-concrete was conducted at the component levels of the SGICF walls. Six simply supported reinforced concrete beams ( $3 \mathrm{ft}$. long) were tested in a three-point bending configuration by applying a cyclic loading protocol. Results showed that the steel fiber-concrete did not improve the lateral strength and the drift capacity. Steel fiber dose played an essential role in this investigation.

Analytical approaches were used to estimate the initial stiffness and the lateral strength of the SGICF walls. None of the approaches managed to provide an acceptable estimation of the initial stiffness. Meanwhile, the summation of individual vertical core plastic moment capacity and the ACI equation for the minimum shear strength of the concrete methods were successful in estimating the in-plane lateral strengths. Finally, a computer model was created to predict the lateral in-plane behavior of the SGICF walls. Results showed that the computer model provided good estimations for the peak lateral strength and the initial stiffness. 
To my family 


\section{Acknowledgments}

First of all, all praise to Allah (God) for giving me the strength and patience to complete this work. I would like to give my sincerest gratitude to my advisor, Dr. Peter Dusicka, for all his guidance and support throughout the duration of my Ph.D. studies at Portland State University. His expertise and understanding added considerable value to my graduate experience. I also would like to thank my doctoral committee members, Dr. Franz Rad, Dr. Thomas Schumacher, and Dr. Hormoz Zerah, for taking the time to review my dissertation and provide valuable guidance and comments.

I am grateful to the Ministry of Higher Education and Scientific Research (MOHESR) in Iraq for offering me the opportunity to conduct my Ph.D. studies at Portland State University and provide the financial assistance to conduct the experimental investigations. I also wish to thank my fellow graduate students and friends in the iSTAR Laboratory, in particular, Hosam Al-Azzawi, Hayder Al-Khafaji, Anas Yosefani, Ali Hafiz, Aqeel Al-Bahadily, Naowar Al-Abbas, Ilya Palknikov, Alvaro Lopez, and Aaron Smith for valuable advice, help and productive discussions.

Finally, writing a thesis is not possible without an extremely understanding family, so I must thank my parents, brother, and sisters for their constant support while I was studying. I cannot express in words how grateful I am to my wife, daughter, Shams, and son, Youssif, for their support, love, understanding, and patience during my study. 


\section{Table of Contents}

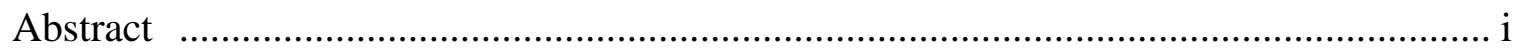

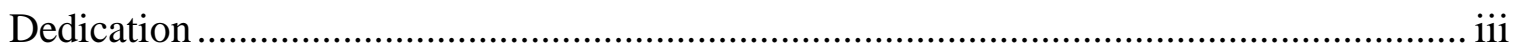

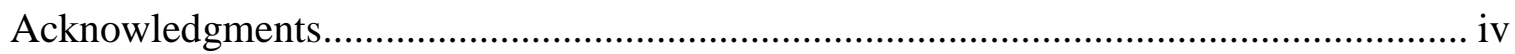

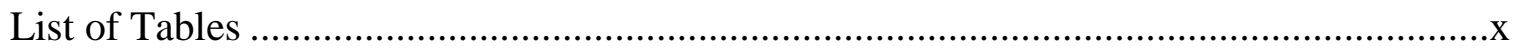

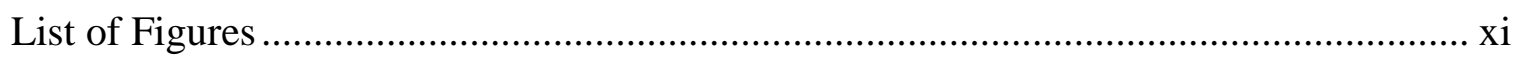

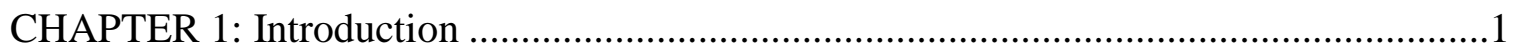

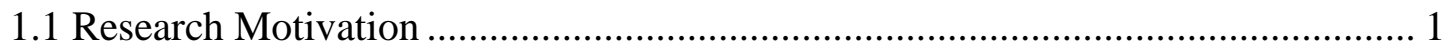

1.2 Research Significance ………………........................................................ 2

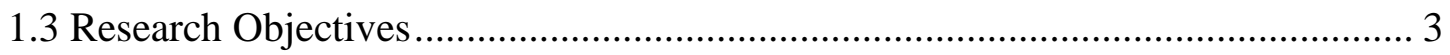

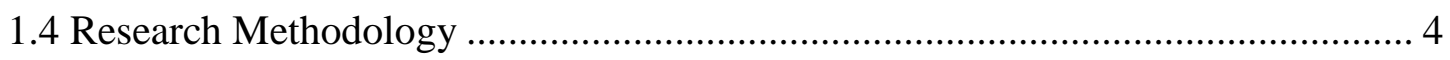

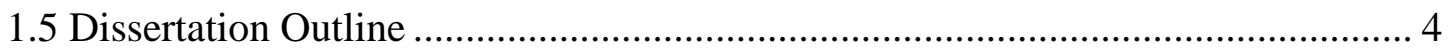

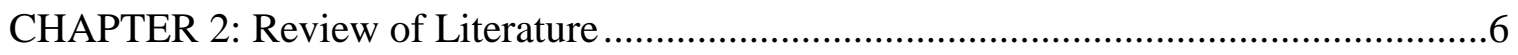

2.1 SGICF Walls under Monotonic Loading ............................................................. 6

2.1.1 Portland Cement Association Experimental Investigation ............................... 6

2.1.2 National Association of Home Builders Study …….......................................... 7

2.2 SGICF Walls under Cyclic Loading ............................................................ 8

2.2.1 Portland State University Studies …………….......................................... 9

2.2.1.1 Dusicka and Kay ………………………........................................... 9

2.2.1.2 Dusicka and Werner........................................................................... 10 


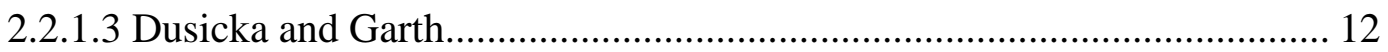

2.2.2 The American University in Cairo Study .................................................... 14

CHAPTER 3: Large Scale Dynamic Experimental Program ...........................................16

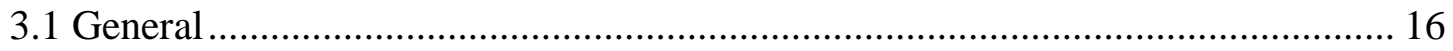

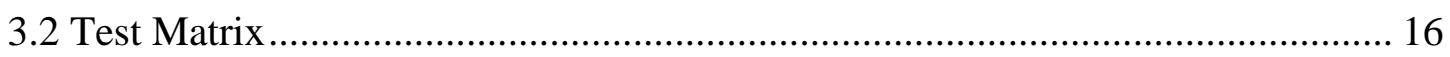

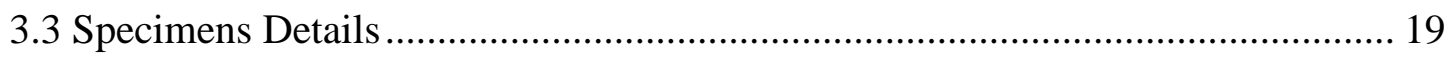

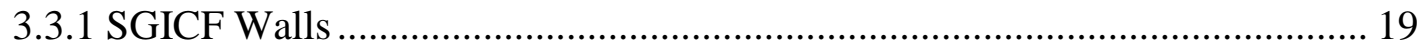

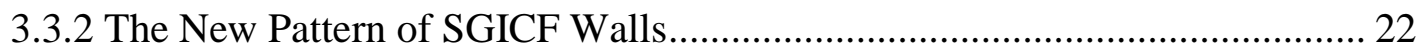

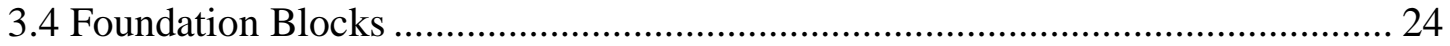

3.5 Material Properties …………………………………................................... 27

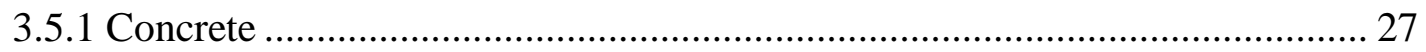

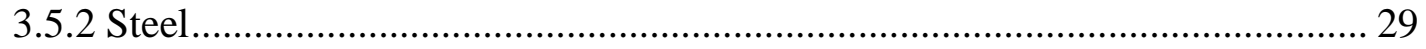

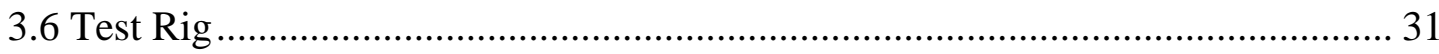

3.7 Ground Motion Selection................................................................................. 34

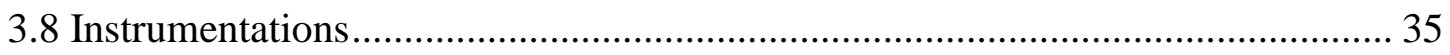

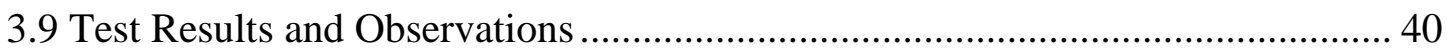

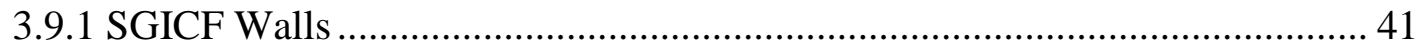

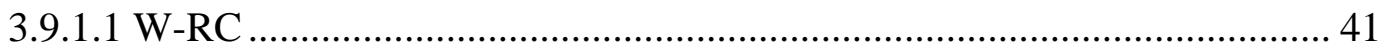

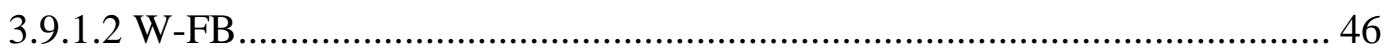

3.9.1.3 Results Summary of SGICF Walls ......................................................... 49 


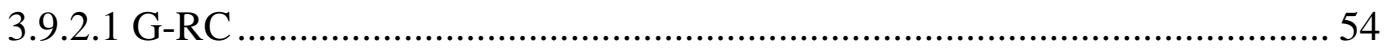

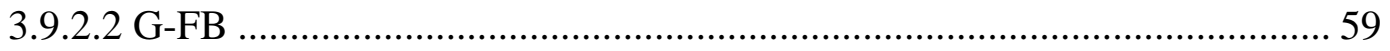

3.9.2.3 Results Summary of The New SGICF Walls ....................................... 65

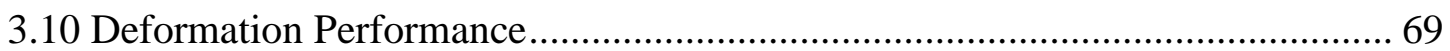

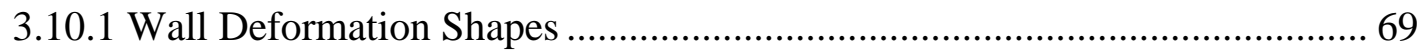

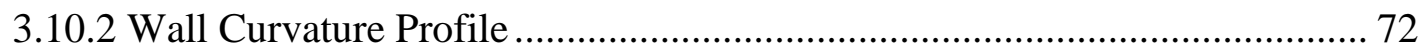

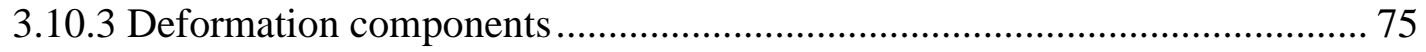

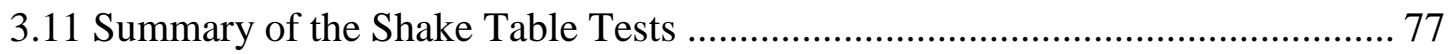

CHAPTER 4: Component Level Cyclic Experimental Program....................................79

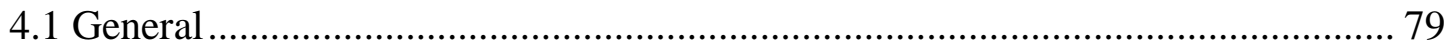

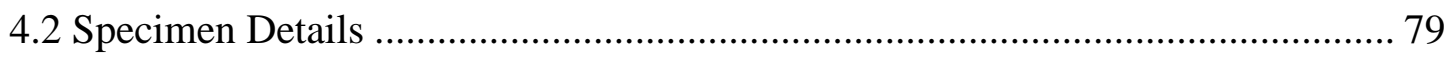

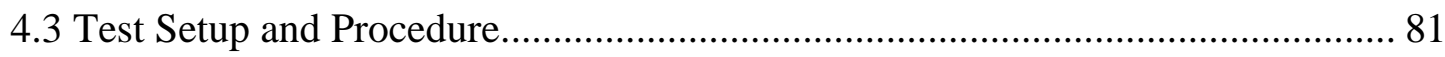

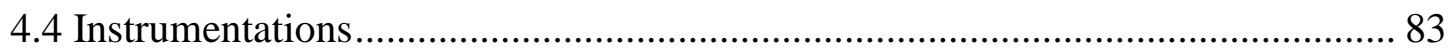

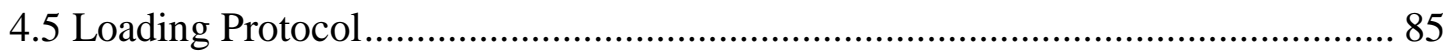

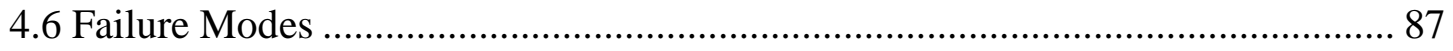

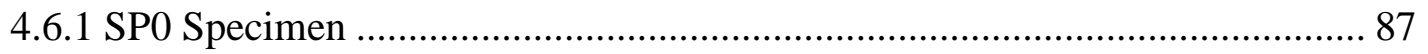

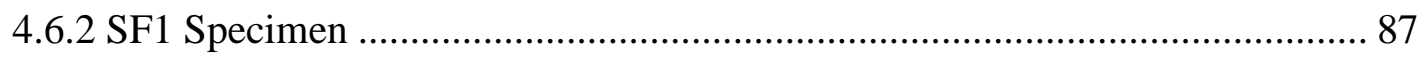

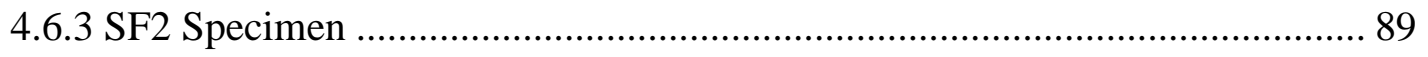

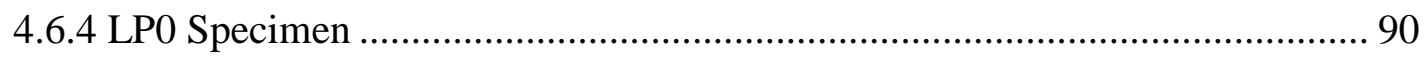




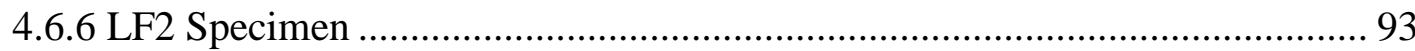

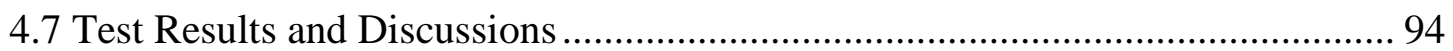

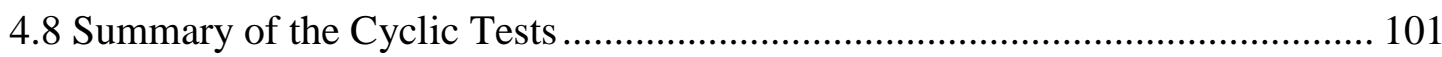

CHAPTER 5: Evaluating Existing Analytical Estimations ..............................................102

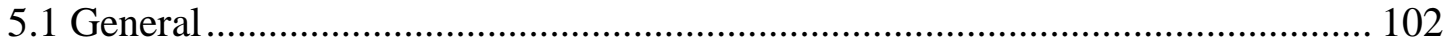

5.2 Estimation of the Initial Stiffness ..................................................................... 102

5.2.1 Hand Calculation Approaches for Solid Shear Walls with Openings ........... 103

5.2.1.1 Solid Shear Wall without Piers Rotation Effect (SOPR) ......................... 103

5.2.1.2 Solid Shear Wall with Piers Rotation Effect (SWPR) ............................ 105

5.2.2 Equivalent Shear Media Method (ESM) ……………................................. 106

5.2.3 Simplified Beam-Column Approach (SBCA) ……………………............. 108

5.2.3.1 Lateral Relative Displacement of the Intermediate Stories .................... 109

5.2.3.2 Lateral Relative Displacement of the Top Story.................................... 110

5.2.3.3 Lateral Relative Displacement of the Bottom Story ............................... 110

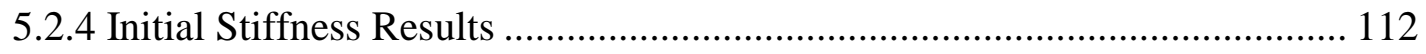

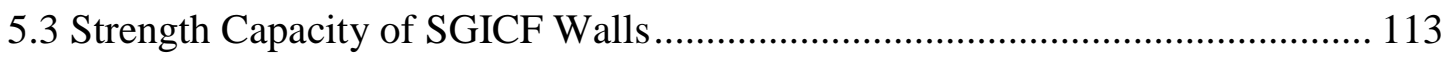

5.3.1 Summation of Individual Vertical Core Plastic Moment Capacity ( $\left.\sum \mathrm{V}_{\mathrm{cm}}\right) 114$

5.3.2 Consideration of Reinforced Vertical Concrete Cores Only $\left(\mathrm{V}_{\mathrm{C}}\right)$................ 114

5.3.3 Shear Capacity Due to the Plastic Moment of the Whole Wall ( $\left.\mathrm{V}_{\mathrm{Mw}}\right)$....... 115 
5.3.4 Shear Capacity by using ICC ESR 1770 Equations ( $\left.\mathrm{V}_{\mathrm{C}}(\mathrm{ICC})\right)$................... 116

5.3.5 Shear Strength Results ......................................................................... 116

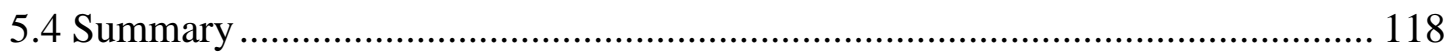

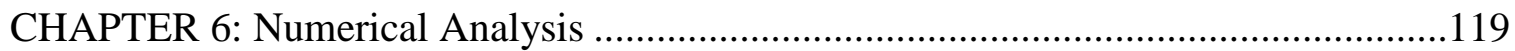

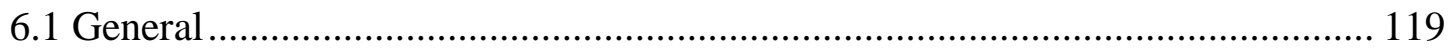

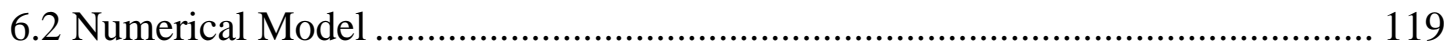

6.2.1 Finite Element Model Description and Materials ..................................... 119

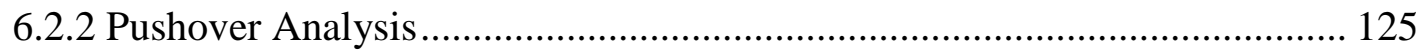

6.2.3 Numerical Analysis Results ......................................................... 126

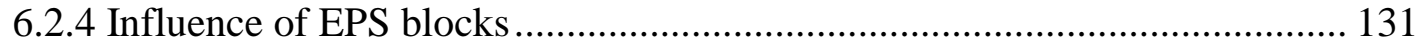

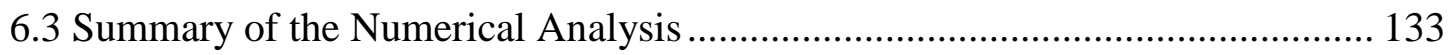

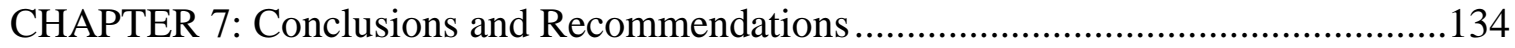

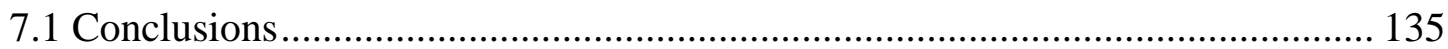

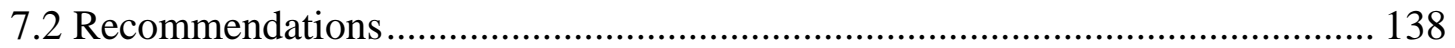

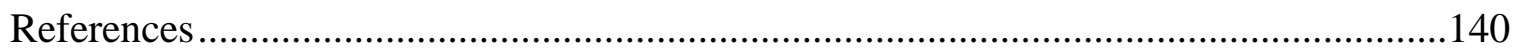




\section{List of Tables}

Table 3-1: Test matrix and designations for each wall test. ..................................... 18

Table 3-2 Average compressive concrete strengths.................................................. 28

Table 3-3 Measured peak performance values for SGICF walls................................. 51

Table 3-4 Measured stiffness of SGICF walls from the free vibration tests. ................... 52

Table 3-5 Measured peak performance values for the new pattern SGICF walls. ........... 67

Table 3-6 Measured stiffness of the new pattern SGICF walls from the free vibration

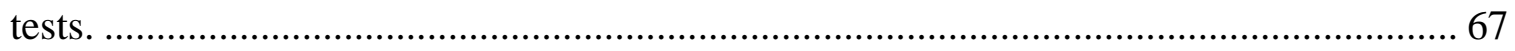

Table 4-1 Test matrix with beams designation......................................................... 81

Table 5-1 Comparison of analytical initial stiffness with experimental results............. 113

Table 5-2 Comparison of theoretical peak lateral strength and peak experimental loads.

Table 6-1 Initial stiffness and peak load comparisons between numerical models and

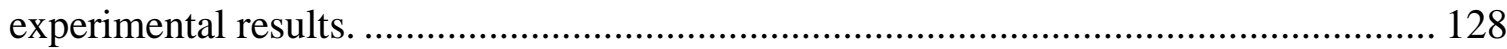




\section{List of Figures}

Figure 3.1 Standard EPS block detail. .................................................................. 20

Figure 3.2 SGICF wall geometry and reinforcement details.................................. 21

Figure 3.3 Construction of the SGICF walls. ........................................................... 21

Figure 3.4 New pattern of SGICF wall geometry and reinforcement details................. 23

Figure 3.5 Construction of the new pattern of SGICF wall....................................... 24

Figure 3.6 SGICF wall foundation block detail........................................................ 25

Figure 3.7 Construction of SGICF wall foundation block....................................... 26

Figure 3.8 New pattern of SGICF wall foundation block detail................................. 26

Figure 3.9 Slump test for the foundation blocks................................................. 27

Figure 3.10 Apparatus used to determine the compressive strength of the concrete........ 29

Figure 3.11 Apparatus used to determine steel reinforcement tensile strength.............. 30

Figure 3.12 Stress-strain curve for \#4 steel reinforcement bar. .................................... 30

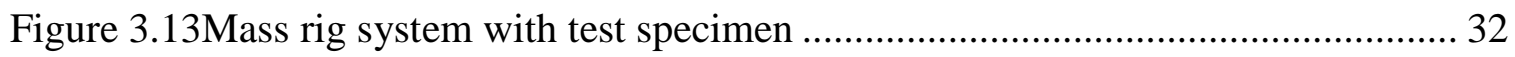

Figure 3.14 SGICF wall with the load beam and load cell....................................... 33

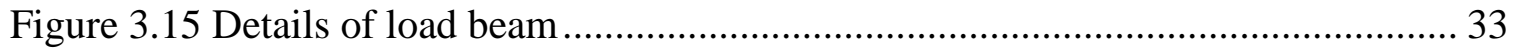

Figure 3.16 Selected ground acceleration: (a) time history, (b) response spectrum......... 34

Figure 3.17 Location of strain gauges for W-RC and W-FB specimens....................... 36

Figure 3.18 Location of LVDTs, string pots, and accelerometers for W-RC and W-FB

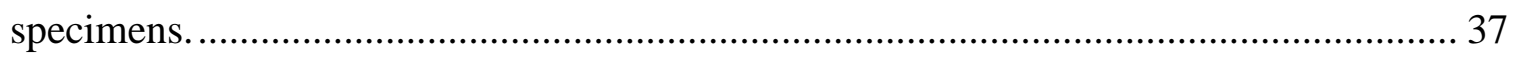

Figure 3.19 Location of accelerometers on the specimen....................................... 38

Figure 3.20 Location of strain gauges for GRC and G-FB specimens......................... 39 
Figure 3.21 Location of LVDTs, string pots, and accelerometers for G-RC and G-FB

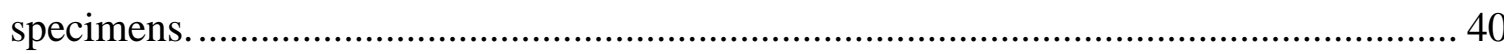

Figure 3.22 Failure mode of W-RC: (a) SFGM-0.3, (b) SFGM-0.5, (c) SFGM-0.75, (d)

SFGM-1.0.

Figure 3.23 Earthquake response for W-RC: (a) SFGM-0.3, (b) SFGM-0.5, (c) SFGM-

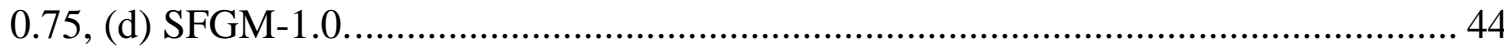

Figure 3.24 Collapse of W-RC at SFGM-1.25 .................................................. 45

Figure 3.25 Hysteresis response of $\mathrm{W}-\mathrm{RC}$ to 1.25 scaled ground motion...................... 45

Figure 3.26 Failure mode of W-FB: (a) SFGM-0.3, (b) SFGM-0.5, (c) SFGM-0.75, (d)

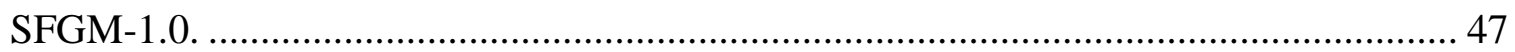

Figure 3.27 Earthquake response for W-FB: (a) SFGM-0.3, (b) SFGM-0.5, (c) SFGM-

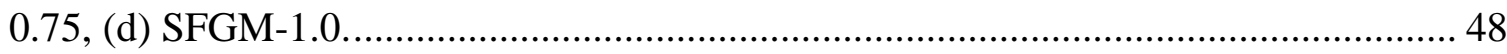

Figure $3.28 \mathrm{~W}-\mathrm{FB}$ specimen with wall forms removed for inspection......................... 49

Figure 3.29 Experimental envelop curves for the SGICF walls................................ 50

Figure 3.30 Backbone curves comparison of the SGICF walls. ..................................50

Figure 3.31 Comparison of the natural period and stiffness of SGICF walls................. 53

Figure 3.32 Hysteresis comparison to 1.0 scaled ground motion of SGICF walls.......... 53

Figure 3.33 Failure mode of G-RC: (a) SFGM-0.3, (b) SFGM-0.5, (c) SFGM-0.75, (d)

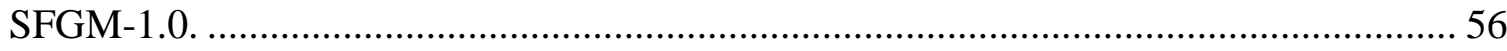

Figure 3.34 Earthquake response for G-RC: (a) SFGM-0.3, (b) SFGM-0.5, (c) SFGM-

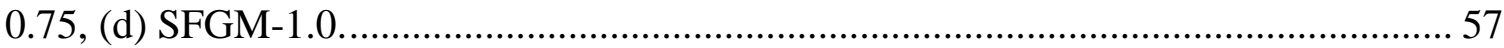

Figure 3.35 Failure mode of G-RC wall at SFGM-1.25 ........................................ 58

Figure 3.36 Hysteresis response of G-RC wall to 1.25 scaled ground motion................. 58 
Figure 3.37 Lack of concrete consolidation in G-FB specimen. 60

Figure 3.38 Failure mode of G-FB: (a) SFGM-0.3, (b) SFGM-0.5, (c) SFGM-0.75, ..... 62

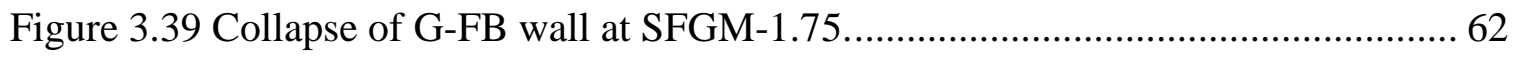

Figure 3.40 Earthquake response for G-FB: (a) SFGM-0.3, (b) SFGM-0.5, (c) SFGM-

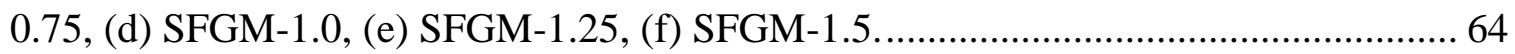

Figure 3.41 Hysteresis response of G-FB wall to 1.75 scaled ground motion................. 64

Figure 3.42 Experimental envelop curves for G-RC and G-FB walls............................ 65

Figure 3.43 Comparison of the natural period and stiffness of the new pattern of SGICF

walls. 66

Figure 3.44 Hysteresis comparison to 1.25 scaled ground motion for the new pattern

SGICF walls. 68

Figure 3.45 Backbone curves comparison of the new pattern SGICF walls. ................... 68

Figure 3.46 Deformation shapes for all tested SGICF walls......................................... 70

Figure 3.47 Curvature profile along the wall height for all tested SGICF walls............. 74

Figure 3.48 Deformation componenets contribution ratio............................................. 78

Figure 4.1 Construction of the beam specimens......................................................... 80

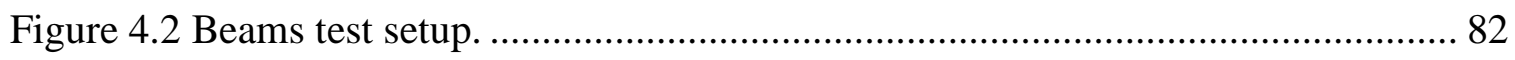

Figure 4.3 Instrumentation layout for set no.1 beams. ................................................. 84

Figure 4.4 Instrumentation layout for set no.2 beams. ................................................ 84

Figure 4.5 Loading protocol for the first set of the beams............................................. 86

Figure 4.6 Loading protocol for the second set of the beams......................................... 86

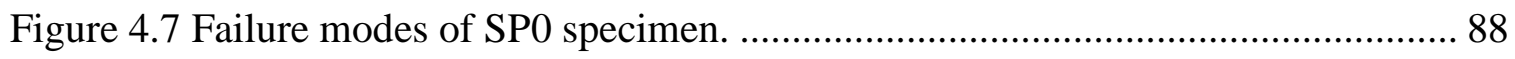

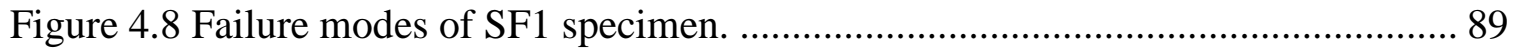




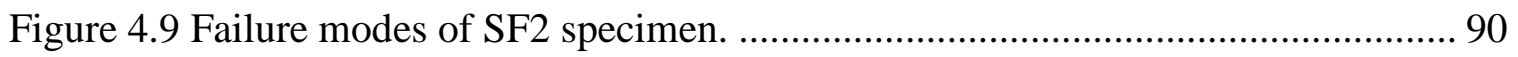

Figure 4.10 Failure modes of LP0 specimen. ...................................................... 91

Figure 4.11 Failure modes of LF1 specimen. .................................................... 92

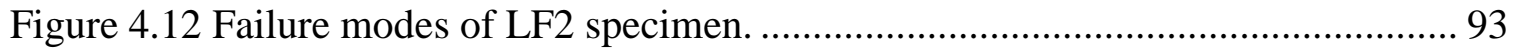

Figure 4.13 Hysteresis curves for the first set of the beams. ...................................... 95

Figure 4.14 Load-displacement backbone curves for the first set of the beams.............. 96

Figure 4.15 Moment curvature backbone curves for SF1 and SF2 specimens (west side).

Figure 4.16 Moment curvature backbone curves for SP0 and SF2 specimens (east side).

Figure 4.17 Hysteresis curves for the second set of the beams.

Figure 4.18 Load-displacement backbone curves for the second set of the beams....... 100

Figure 4.19 Moment curvature backbone curves for LP0 and LF2 specimens (east and

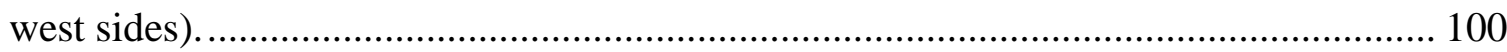

Figure 5.1 Solid shear wall without pier rotation effect. ........................................ 104

Figure 5.2 Solid shear wall with pier rotation effect. ........................................... 106

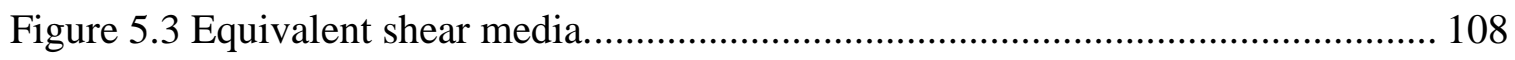

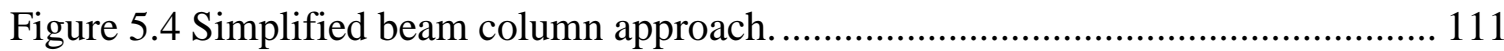

Figure 6.1 Compression concentric struts analogy .......................................... 121

Figure 6.2 Compression tests of EPS material (Werner, 2010)................................ 122

Figure 6.3 Nonlinear stress-strain curve for EPS block used in the numerical model. .. 123 Figure 6.4 Nonlinear stress-strain curve for concrete (W-RC wall) used in the numerical model 124 
Figure 6.5 Nonlinear stress-strain curve for steel rebar used in the numerical model.... 124

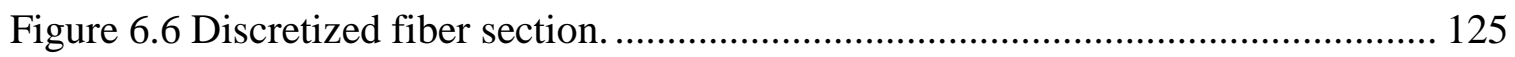

Figure 6.7 Comparison of experimental and numerical load-displacement curves....... 127

Figure 6.8 Fiber plastic hinges reinforcement yield progress mechanism for W-RC wall.

Figure 6.9 Fiber plastic hinges reinforcement yield progress mechanism for W-FB wall.

Figure 6.10 Fiber plastic hinges reinforcement yield progress mechanism for G-RC wall.

Figure 6.11 Fiber plastic hinges reinforcement yield progress mechanism for G-FB wall. 130

Figure 6.12 Numerical models: (a) W-RC without EPS struts, (b) G-RC with EPS struts.

Figure 6.13 Comparison of numerical models results for W-RC and G-RC specimens. 132 


\section{CHAPTER 1: Introduction}

\subsection{Research Motivation}

Modern countries develop the best methodologies to construct new buildings that maintain a high level of sustainability and performance. In the United States, these guidelines have been set by the Leadership in Energy and Environmental Design (LEED) system that was developed by the United States Green Building Council (USGBC) (Ochieng, et al., 2014). The production of construction materials, concrete, steel, and wood, has contributed to the increase of greenhouse gases (such as dioxide carbon gas $\mathrm{CO}_{2}$ ), which contribute to global warming. Therefore, new building systems that are more energy efficient, and reduce usage of natural resources, have become the most adopted and successful alternatives.

Insulated Concrete Form (ICF) walls are building components that are primarily used in the residential construction industries that strive for more sustainable buildings. Although there are several different types of ICFs (e.g., screen-grid, waffle-grid, and flat), the principles behind each remain the same; they are stay-in-place forms that contain reinforced concrete. The presence of the ICF wall forms reduces the amount of concrete relative to a conventional reinforced concrete wall, especially for screen-grid insulated concrete form (SGICF) walls. Buildings constructed with SGICF walls also have other positive environmental benefits, such as healthier indoor environment, higher reuse of recycled materials, and a more energy efficient building envelope due to the high R-value and thermal mass of the blocks walls (Sultan, 2016). Each SGICF form has 
horizontal and vertical cutouts that when stacked create internal hollow grids that are filled with concrete and reinforcing steel. The ICF form blocks can be made from different recycled materials, such as recycled wood chips and expanded polystyrene foam, which are bonded together with cement. Because of the reinforced concrete grid within SGICF walls, the structural behavior of the walls is neither a shear wall nor a frame system and needs more investigation to qualify these walls for applications outside of residential construction.

\subsection{Research Significance}

Monotonic and cyclic experiments had been conducted to assess the lateral response of ICF walls (NAHB Research Center,Inc., 2001) \& (Dusicka \& Kay, 2011). Since various commercially available ICF blocks made from recycled materials with different cross section cores, such as circular and rectangular shapes, each type requires investigation to assess the overall strength and durability. Experimental investigation of SGICFs at a component level, both circular and rectangular configurations, was conducted to understand their in-plane lateral force better and drift ratio capacities (Dusicka \& Werner, 2012). In these components level experiments, it was found that the strength of the ICF walls was improve by up to $100 \%$ and lateral deformation capacity by up to $60 \%$ due to the presence of the ICF blocks.

Furthermore, the research demonstrated the potential benefits of using fiber reinforced concrete for the cores. Experiments for the in-plane behavior at full-scale SGICFs walls had also been found to be influenced by the presence of ICF blocks, again 
resulting in higher strength as compared to reinforced concrete grids without the ICF blocks (Dusicka \& Kay, 2011). All of these investigations used cyclic loading in evaluating the in-plane lateral resistance of ICF walls.

In order to provide valuable information about the use of the SGICF walls in areas that are prone to seismic events, an experimental investigation was conducted. Four different SGICF walls were subjected to a real-time earthquake dynamic loading through the use of shake table tests, which are designed to investigate the in-plane lateral response of these walls. Steel fiber-reinforced concrete was used to evaluate the anticipated improvements to the lateral strength and the lateral displacement capacity of the SGICF walls. Furthermore, an experimental investigation at the component level was conducted on six circular beams by applying cyclic loading to evaluate the influence of the steel fiber concrete and the improvement in the failure modes of the new SGICF walls.

\subsection{Research Objectives}

The main objectives of the study are as follow:

$>\quad$ Experimentally evaluate the SGICF walls by applying real-time history dynamic loading.

$>\quad$ Compare the behavior of SGICF walls constructed with conventional concrete cores to walls that are built with steel fiber-reinforced concrete cores.

$>\quad$ Suggest and build new screen grid pattern walls.

$>\quad$ Investigate experimentally, at the component level, bending and shear resistance of individual cores. 
$>\quad$ Assess the available analytical approaches in calculating the initial stiffness and the lateral strength of the SGICF walls, and decide the most efficient method that can provide suitable estimates.

$>\quad$ Validate the suggested numerical models based on experimental results in an effort to provide researchers and designers with a useful tool to evaluate the dynamic behavior of the SGICF walls.

\subsection{Research Methodology}

The research was divided into five main parts. First, a literature review was carried out to evaluate the current experimental investigations on the SGICF walls that used different loading applications. Second, dynamic properties and lateral strength of the SGICF walls subjected to a real-time history dynamic loading were experimentally evaluated by using shake table tests. Third, a shear and bending moment evaluation of the SGICF wall cores at the component level was conducted by applying a quasi-static loading. Fourth, the initial stiffness and the lateral strength of the SGICF walls were analytically evaluated. Finally, nonlinear modeling of the SGICF walls was carried out to evaluate the experimental results and provide a suitable numerical representation of these walls.

\subsection{Dissertation Outline}

The dissertation is organized into seven main chapters with the following contents: 
Chapter 1 discusses the motivation of the study, scope of work, research objectives, and research methodology.

$>\quad$ Chapter 2 presents literature review on the previous experimental investigations conducted on the ICF walls with an emphasis on the applied loading type.

Chapter 3 presents the experimental evaluation of the large-scale SGICF walls using time history dynamic loading. Details of the foundation blocks, SGICF walls, and the new suggested walls, mass rig, and the selected ground motion are presented. Later, failure modes and test results are discussed.

$>\quad$ Chapter 4 presents the experimental evaluation of SGICF walls cores at the component level using cyclic loading protocols that aim to evaluate the influence of the steel fiber-reinforced concrete on the lateral strength of the SGICF walls. With that aim, six 3-ft circular specimens are tested. In this chapter, failure modes and tests results are discussed.

$>\quad$ Chapter 5 presents analytical methods to estimate the initial stiffness and lateral strength of the SGICF walls.

$>\quad$ Chapter 6 presents numerical simulations of the SGICF walls based on the experimental results of walls materials presented in Chapter 3. Numerical simulations of the SGICF walls are conducted using SAP2000.

Chapter 7 summarizes the observations and conclusions drawn based on the experimental, analytical, and the numerical results of this study. 


\section{CHAPTER 2: Review of Literature}

ICF walls went through various experimental and analytical evaluations to assess the overall lateral strength, energy dissipation, ductility, and stiffness degradation. Prior research efforts have investigated the ICF wall's in-plane lateral strength by using different loading protocols. The following studies represent the most recent researches on SGICF walls.

\subsection{SGICF Walls under Monotonic Loading}

Two studies were found in the literature that investigated different aspect ratios (hight/length) of SGICF walls in-plane lateral strength by applying monotonic loading.

\subsubsection{Portland Cement Association Experimental Investigation}

Screen grid insulated concrete form (SGICF) walls were experimentally investigated by Mehrabi in 2000 for the Portland Cement Association (PCA) (Mehrabi, 2000). The author compared wood-frame, steel-frame, ICF flat wall, ICF waffle-grid wall, and ICF screen-grid wall panels. All tested walls were designed to have the same aspect ratio (height/length) of 2 . The 8 -ft tall $x$ 4-ft wide walls were tested for in-plane lateral load resistance, which was designed based on the recommendations and guidelines for typical exterior wall panels in earthquake zones 1 or 2 and $70 \mathrm{mph}$ minimum wind speeds. Only one wall of each type was tested by applying monotonic static in-plane loads from a hydraulic ram. 
The test results showed that SGICF walls are stronger and stiffer than wood-frame or steel-frame wall panels. The initial stiffness of the SGICF wall panels was 18 and 38 times higher than the wood-frame or steel-frame wall panels. Furthermore, testing results revealed that the SGICF wall exhibited a diagonal cracking pattern, which extended down from 29 in above the footing on the loaded side to the footing/wall intersection on the opposite side. The maximum displacement of $1.7 \mathrm{in}$., which is a $1.77 \%$ drift ratio, and the maximum in-plane lateral force of 27.9 kips were observed during the test. The screen-grid wall was up to 6.5-8 times stronger, and stiffer than the wood-frame and the steel-frame panel walls of the identical dimensions.

\subsubsection{National Association of Home Builders Study}

The National Association of Home Builders (NAHB) (NAHB Research Center,Inc., 2001) conducted an experimental investigation on the ICF walls to study the influence of height-to-length aspect ratio, openings in windows and doors, and the minimal reinforcement details provided by the prescriptive method for the insulating concrete forms in residential construction (NAHB Research Center,Inc, 1998). The researchers investigated three types of defined ICF walls: flat, waffle, and screen grid. Two aspect ratios 4:1 and 2:1 were considered in this investigation with openings that simulate window or garage door openings. Total of nine ICF walls were subjected to monotonic loading from a hydraulic actuator, which was connected to the top of each wall and applied one-directional displacement with a constant rate and a maximum displacement of $4 \mathrm{in} .$. 
The NAHB experimental investigation objectives were to find the aspect ratios of ICF walls that determine the shear and flexural failures, and the locations of these failures. Moreover, the investigation aimed to compare the shear strength obtained from the experimental investigation with the guidelines of the prescriptive method in predicting ICF walls lateral strength.

Experimental results of the in-plane lateral strength of the SGICF walls were compared to the results that were calculated from the two analytical models. The comparison revealed that the experimental shear capacity of the SGICF wall, which was without openings, was higher than the predicted once. Contradictorily, the experimental lateral strength results of SGICF walls that have openings were lower than that obtained from the analytical methods. Because only one sample of each ICF wall type was tested, the NAHB researchers suggested that more investigations are required to investigate multiple samples of the same specimens for failure modes evaluation, and to verify the equations used to estimate their lateral strength.

\subsection{SGICF Walls under Cyclic Loading}

Several studies have been conducted on SGICF walls to investigate the seismic performance of various types of ICF walls. The following investigations applied the quasi-static approach for the loading application. 


\subsubsection{Portland State University Studies}

Several studies have been conducted at Portland State University to investigate the seismic behavior of SGICF walls in the infrastructure Testing and Applied Research (iSTAR) laboratory.

\subsubsection{Dusicka and Kay}

An experimental study was conducted by Peter Dusicka and Thomas Kay (Dusicka \& Kay, 2011) to investigate the in-plane lateral behavior of screen grid insulated concrete form walls (SGICF). Thirteen full-scale walls were constructed with two different aspect ratios $(\mathrm{H} / \mathrm{L}=0.89$ and 2.67$)$, and two different vertical loading conditions (walls under self-weight only and walls under an additional externally applied distributed vertical load of $10,000 \mathrm{lb} / \mathrm{ft}$ ). The goal of using two types of the vertical loads was to perceive the performance of the ICF walls with and without additional load bearing demands. Four groups of ICF full-scale walls were established; each group consisted of three walls with the same specifications. Also, one low aspect ratio nominal reinforced concrete wall was constructed as a reference specimen for results comparison between SGICF and reinforced concrete walls.

SGICF walls were built on the top of a reinforced concrete foundation to simulate the construction building field. Reinforcement dowel bars \#5 were extended above the foundation of the walls and linked to the vertical rebars of the SGICF walls to prevent foundation failure. All of the walls had the same reinforcement ratio (United \#4 reinforcing bars at each horizontal and vertical opening), and were tested after 28 days of 
curing. The tested walls were restrained against out-of-plane movement by using two lateral restraint struts. A \pm 220 kip hydraulic actuator with a stroke capacity of \pm 20 in. was used to apply the cyclic loading. Linear displacement transducers were attached at different locations to record the in-plane walls deformation.

Many conclusions were made regarding the behavior of SGICF walls against the in-plane lateral cyclic loading. When comparing the failure modes of SGICF walls that carried vertical self-weight of high aspect ratio to those of low aspect ratio groups, both of the groups showed that most of the damage occurred in the outer vertical cores. Meanwhile, minimal damages were observed in the horizontal cores. A significant increase in strength was observed when applying the vertical loads to both aspect ratios walls, and there was a delay at the beginning of strength deterioration. The ICF blocks increased the strength of the walls by $20 \%$ even though their compressive strength was near to $1 \%$ of compressive strength, especially for the walls with a low aspect ratio. Finally, the authors noted that the effective stiffness of the walls was higher with the applied vertical load than with the walls carrying self-weight only. However, the residual stiffness decreased rapidly during the repeated lateral load cycles.

\subsubsection{Dusicka and Werner}

Peter Dusicka and Carl Werner (Dusicka \& Werner, 2012) conducted the second experimental program to provide potential strategies for improving the hysteretic response of screen grid insulated concrete form walls at the components level. Two strategies were adopted to increase the ability of SGICF walls to resist the in-plane cyclic 
loading, either by inserting wire mesh into the ICF forms opening before pouring the concrete or by adding fiber reinforcement to the concrete mix.

Two types of SGICF blocks were used. The first block had dimensions of 48x16x10 in. with interior 6 in. diameter hollow cores that ran vertically and horizontally every 16 in. when stacked with other blocks that were manufactured from recycled polystyrene. For this type, the final specimen section dimension resulted from stacking two blocks was $32 \times 35.5$ in., taking in consideration load frame dimension. The second ICF block, manufactured from crushed and mineralized recycled pallet wood cementitiously bonded together, was with aspects of $24 \mathrm{in.} \mathrm{length,} 8 \mathrm{in.} \mathrm{high} \mathrm{and} 12 \mathrm{in.}$ thick. An interior concrete screen grid with vertical rectangular concrete cores of 5 in. deep and 9 in. wide columns with 2 in. spacing between each consecutive cores was constructed by stacking the ICF blocks. Horizontal cores with $4 \times 4$ in. was generated by stacking two blocks vertically. The final specimen section dimension was 24 in. wide and 32 in. tall. For both types, a single \# 4 reinforcing rebar was placed in the center of each core.

After applying three full cyclic load reversals using the hydraulic actuator of 100 kip force capacity and \pm 3 in. stroke, many conclusions were made based on the type of block, and the in-plane lateral strength improvement techniques. The authors observed a generation of flexural cracks on both specimen section types at both ends of the vertical core that used wire mesh as a developing technique. Also, vertical core failure was not achieved. Meanwhile, authors did not observe the ultimate failure of the specimens that used fiber concrete as an improving technique because the tests stopped when the linear 
variable differential transformer reached the end of its stroke. As a result, the authors concluded that using polyvinyl alcohol fibers increased the shear strength of the concrete mix in both specimen types. They recommended the testing of full-scale walls to investigate the behavior of the SGICF walls, and to overcome laboratory equipment limitation to measure the exact strength of the vertical cores improved by polyvinyl alcohol fibers.

The authors reported that both types (circular core and rectangular core blocks) achieved similar strengths, and proposed that the use of different kinds of blocks did not add any significant lateral strength to the vertical cores. Finally, the wire mesh technique showed considerable improvement of the ductility of the circular core as compared to the specimens with a rectangular core that showed a moderate effect on vertical cores strength when using confinement of round wire mesh.

\subsubsection{Dusicka and Garth}

Peter Dusicka and John Garth (Garth, 2014) conducted the third experiment to investigate the performance of newly invented ICF blocks that were made from $85 \%$ of crushed and mineralized waste pallet wood with $15 \%$ cementitious material molded into the blocks. A quasi-static cyclic loading protocol was applied on two different aspect ratios (approximately 2:1 and 1:1) with different axial loading conditions to measure the ICF wall's lateral loading resistance capacity, drift capacity, ductility, energy dissipation, residual stiffness, and the contribution of the ICF blocks to the overall strength. 
Six full-scale SGICF walls were constructed and differentiated into two groups based on the aspect ratio of each wall, which was H/L: 1.74, 0.87. Each group was categorized into three types of walls: a wall without additional gravity loading, a wall with 10 klf additional gravity loading, and a wall with ICF blocks removed to measure ICF blocks strength contribution. The authors emphasized that one SGICF wall had an aspect ratio of 1.98 , which was included in this study due to preliminary design that gave reasonable failure mechanism.

All walls were constructed on a firm foundation to avoid any possibility of failure due to weak foundation capacity, with \#5 rebar dowels extended out 30 in. above the foundation block. Each wall had vertical and horizontal reinforcements of \# 4 rebar that were placed in each core. The same concrete mix was used in all walls with a specified strength of 3000 psi at 28 days. The drift-controlled loading protocol was applied using a 110-kip hydraulic actuator with a maximum static stroke of $\pm 11 \mathrm{in.}$.

After applying the reversal cyclic drift-controlled loading protocol, many failure modes were observed, and several conclusions were drawn. On average, the ICF blocks increased the shear strength of the walls, when left in place, by $28 \%$ and $56 \%$ for walls with high and low aspect ratios, respectively. Also, these forms contributed to the drift capacity of these walls by $90 \%$ (for high aspect ratios) and 114\% (for low aspect ratios). Finally, due to ICF blocks, an increase in the idealized ductility ratio increased by $59 \%$ for high aspect ratio walls and $76 \%$ for low height-to-length ratio walls.

The addition of a $10 \mathrm{klf}$ axial load made significant contributions to the performance of the SGICF walls. The low aspect ratio walls saw a $21 \%$ strength increase, 
while the high aspect ratio walls saw a $42 \%$ strength increase. The additional gravity load also increased the drift capacities of the low and high aspect ratio walls by $13 \%$ and $25 \%$, respectively.

\subsubsection{The American University in Cairo Study}

A study was conducted by Abdel-Mooty et al. (Abdel-Mooty, Haroun, ElMaghraby, Fahmy, \& Abou-Zeid, 2010) with regard to the performance of the screen grid insulated concrete form walls under the combined in-plane vertical and lateral loads. Ten full-scale walls were tested, and the effect of various parameters on the behavior of each wall, such as lateral load capacity, lateral displacement, and modes of failure, was presented. The experimental study investigated the performance of the SGICF walls system in resisting the combined in-plane vertical and lateral loads to simulate the seismic action, and the effect of the different design criteria. A selection of different dowels arrangements was employed to overcome the weak connection problem between the walls and at the base that affected the walls' resistance to the lateral loads.

Ten full-scale SGICF walls were modeled with various specifications. Eight walls were designed to have $3.28 \times 6.56 \mathrm{ft}$. dimension with 6 in. thickness and grid spacing of 10 in. center to center except for one wall that has a grid spacing of $15.75 \mathrm{in}$. center to center. The other two specimens were designed to have $6.56 \times 6.56 \mathrm{ft}$. dimension with 6 in. thickness and 10 in. grid spacing with two types of opening: the first wall had a door opening of $3.28 \times 4.67 \mathrm{ft}$. and the second had 3.28x3.28 ft. window opening. Different reinforcement sizes were used to measure their influence on SGICF wall's lateral 
resistance. A reinforced concrete base beam of $1.3 \times 1.3 \times 7.9 \mathrm{ft}$. was used for each wall to anchor the walls to the test floor, and to prevent the possible horizontal sliding or uplift. The application of the vertical loads was evenly distributed by casting a reinforced concrete beam of $0.5 \times 0.66 \times 3.28 \mathrm{ft}$. on top of each wall.

The adoption of different reinforcement patterns was implemented to investigate the reinforcement effect on each wall's strength. Therefore, the study used either \#4 or \#5 rebar for each grid member and \#5 double dowels. Also, the impact of steel fibers on SGICF walls was investigated by adding steel fibers $\left(3.37 \mathrm{lb} / \mathrm{yd}^{3}\right)$ to the concrete mix of one wall.

The authors concluded that the \#5 double dowels that connected the SGICF walls to the base increased the walls' stability and resistance to the lateral forces. Applying additional vertical loads influenced the lateral strength of the SGICF walls and the shape of failure modes. Increasing the reinforcement size from \#4 to \#5 doubled the lateral strength of the walls. Finally, using steel fibers with a concrete mix increased lateral load capacity by $25 \%$. 


\section{CHAPTER 3: Large Scale Dynamic Experimental Program}

\subsection{General}

Four large-scale SGICF walls were prepared and tested to investigate the dynamic properties and lateral strength of SGICF walls under seismic loading. The SGICF walls were divided into two sets, each set was consisted of two walls with different concrete types. Reinforced concrete was assigned to one of the walls, and steel fiber-reinforced concrete was appointed to the second wall. The first set consisted of two SGICF walls that were constructed by using expanded polystyrene (EPS) blocks. The second set of SGICF walls was designed and built according to the newly suggested improvements. This section first describes the test matrix, the construction of the SGICF walls, the foundation block details, and the properties of the materials used. It then presents the experimental setup and the instrumentations used to monitor the state of the walls during loading, and finally presents and discusses the results obtained.

\subsection{Test Matrix}

The test matrix was developed to consider several variables: performance of the ICF walls with using reinforced concrete and steel fiber-reinforced concrete, the effect of increasing the length of horizontal cores on wall performance, and the influence of using two reinforcement bars in each vertical core on the lateral drift capacity of the wall.

Previous research (Dusicka \& Werner, 2012) had shown promising improvement in the lateral strength and ductility of SGICF walls at the elemental component levels through 
using fiber reinforced engineered cementitious composite (ECC) concrete. Therefore, Helix twisted micro-rebar was considered in this investigation to measure the improvement on both lateral strength and drift capacity of SGICF walls.

An experimental investigation was published by The New South Wales University (Chang, 2014) regarding the use of Helix twisted micro-steel reinforcement in circular concrete piles. It was shown that the fiber used in this investigation improved both the flexural and shear strength of the circular piles. Further, the experimental investigation results showed good agreement with the design moment and shear capacities calculated based on the Helix method (UES ER-0279) (Polytrox,LLC d.b.a Helix Steel, 2016) that was used to determine the required dose. To investigate the effect of using Helix twisted micro-steel reinforcement in the ICF walls, two walls were built out of steel fiber-reinforced concrete with a steel fiber dose of $20 \mathrm{lb} / \mathrm{yd}^{3}$. The Helix 5-25 Micro-Rebar fiber dose was calculated based on the recommendations provided by the evaluation report UES ER-0279.

Another experimental investigation conducted on ICF walls (Garth, 2014) had shown high shear demand on the horizontal cores of the ICF walls. Therefore, the length of the horizontal cores in the newly suggested pattern of ICF walls was increased from 16 in. to 24 in. The increase of the spacing between the vertical cores was designed to have the same length between the outermost vertical cores that was 54 in., which agree with the ICF walls that were built out of EPS blocks. As a result, the total number of the vertical cores in the newly suggested pattern walls was three. Meanwhile, four cores were generated in the ICF walls built from EPS blocks. 
Most of the previous research reported that the vertical cores either have one rebar ineach vertical core or an alterantive pattern of placing the rebars followed, i.e., vertical reinforcing placed in every other core. Only one research (Werner, 2010) investigated the influence of placing two rebars in the EPS blocks at the component level. Werner found that cores with dual reinforcement showed improvement in the energy dissipation and better stiffness when compared to the cores with single reinforcement. The influence of placing more than one rebar in each vertical core on the lateral drift capacity of largescale SGICF walls was investigated by building two walls designed to have two rebars in each vertical core.

Table 3-1 shows the test matrix of the SGICF walls experimental investigation. The wall with designation $\mathrm{W}$ refers to the walls that were built from EPS blocks. On the other hand, the newly suggested pattern walls will have a $\mathrm{G}$ designation. The last two letters after the dash sign refer to the type of concrete that was used in each wall. For instance, $\mathrm{RC}$ indicates that the wall has reinforced concrete and FB if the wall has steel fiber-reinforced concrete.

Table 3-1: Test matrix and designations for each wall test.

\begin{tabular}{|c|c|c|c|c|}
\hline Designation & $\begin{array}{c}\text { Height } \\
\text { (in) }\end{array}$ & $\begin{array}{c}\text { Length } \\
\text { (in) }\end{array}$ & $\begin{array}{c}\text { Vertical Core } \\
\text { Reinforcement }\end{array}$ & Concrete Type \\
\hline W-RC & 86 & 64 & 1 & Reinforced Concrete \\
\hline W-FB & 86 & 64 & 1 & Fiber Reinforced Concrete \\
\hline G-RC & 86 & 60 & 2 & Reinforced Concrete \\
\hline G-FB & 86 & 60 & 2 & Fiber Reinforced Concrete \\
\hline
\end{tabular}




\subsection{Specimens Details}

All of the SGICF walls in this investigation were 86 in. height. Each wall consisted of a grid of vertical and horizontal cores that had a circular cross-section of 6 in. diameter. The following section describes the two types of walls used in this investigation.

\subsubsection{SGICF Walls}

The first set of SGICF walls were built out of EPS blocks, which were manufactured from cleaned, recycled EPS (expanded polystyrene) aggregate that was ground and mixed with cement, proprietary acceleration solution and water (ICC Evaluation Service, 2012). The standard EPS block is 10 in. thick, 16 in. high, and 48 in. long. The weight of each specimen is between 52 and 55 pounds in dry condition. Each block has circular vertical and horizontal cores with a diameter of 6 in. When stacked, there are 6 in. diameter interior hollow cores that run both horizontally and vertically at 16 in. aligned to center. The core dimensions of the EPS-block are shown in Figure 3.1. A grid of horizontal and vertical cores was generated by pouring concrete into stacked EPS blocks. One reinforcement bar \#4 was placed at the center of both the vertical and horizontal cores. On top of each wall, an integrated bond beam was cast to facilitate the load transfer from the steel load transfer beam along the top of the wall. The connection between the SGICF wall and the top steel beam was secured through using J-bolts (7/8 in diameter and 12 in long) that were immersed on top of each vertical core, as shown in Figure 3.2. 
Figure 3.3 outlines the procedure used to build these walls, which was based on the standard building practices for ICF walls. Galvanized threaded rods were placed inside the ICF blocks at each core intersections to assist with the positioning of the horizontal rebar at the center of the cores and to mount the instrumentations.
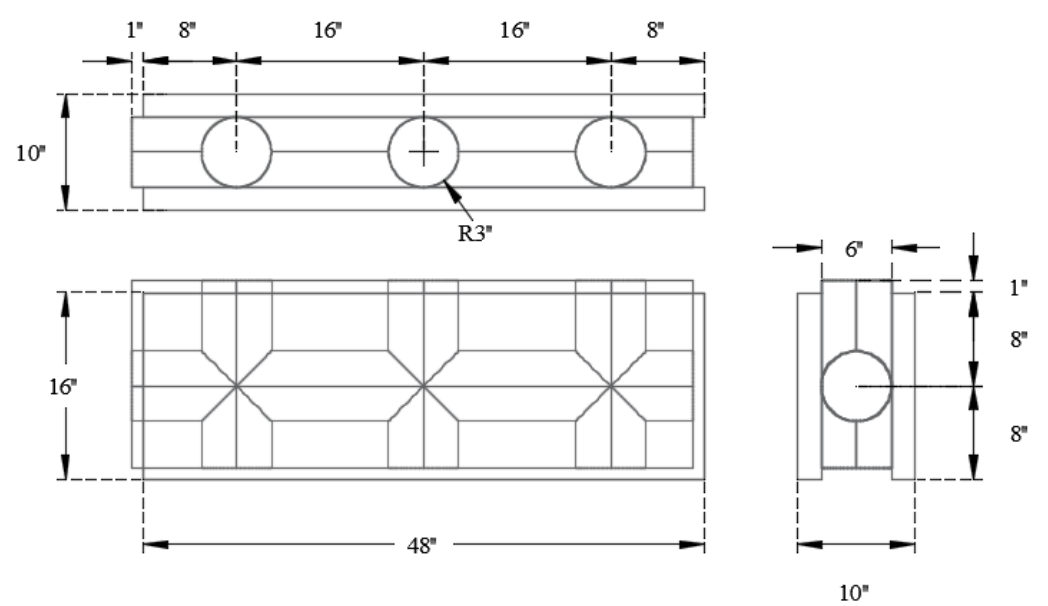

Figure 3.1 Standard EPS block detail. 


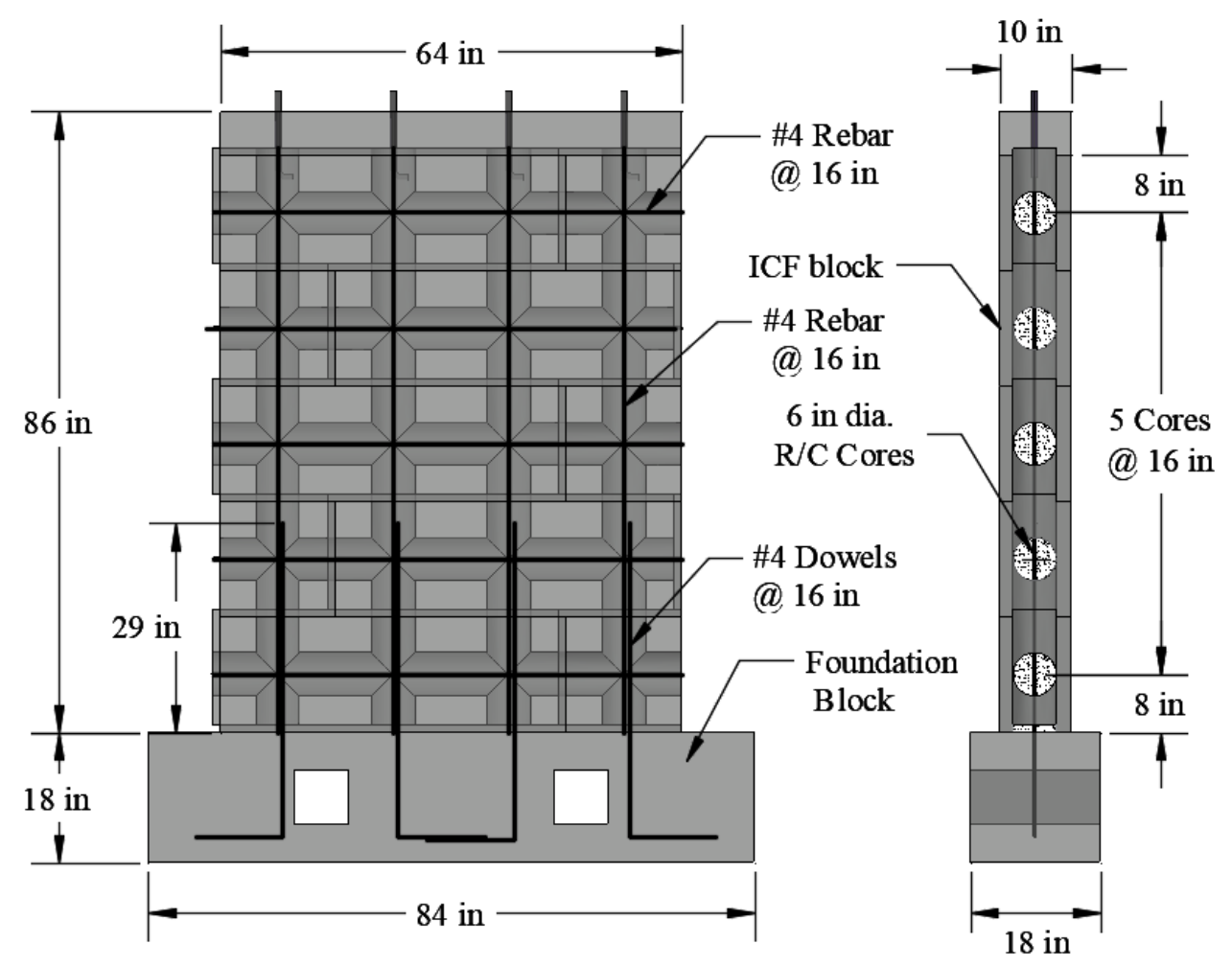

Figure 3.2 SGICF wall geometry and reinforcement details.

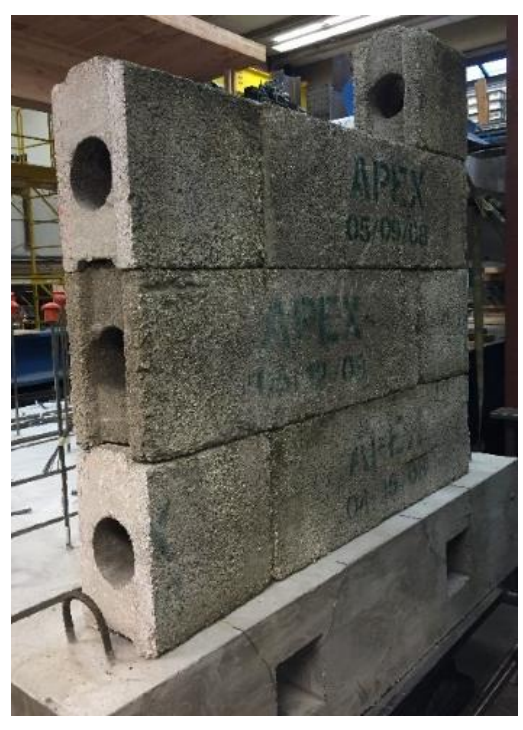

Stacking EPS blocks

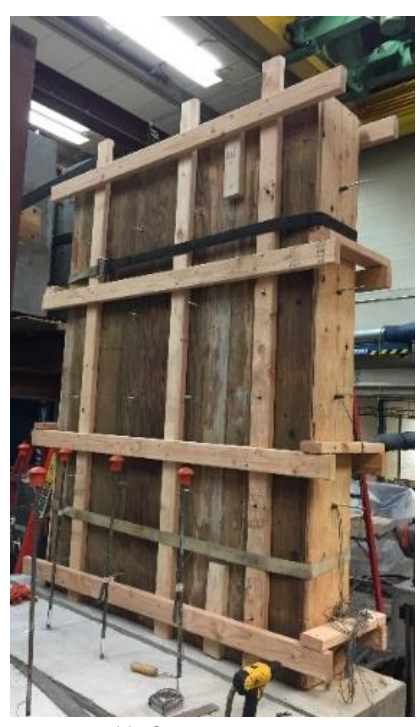

Wall forms

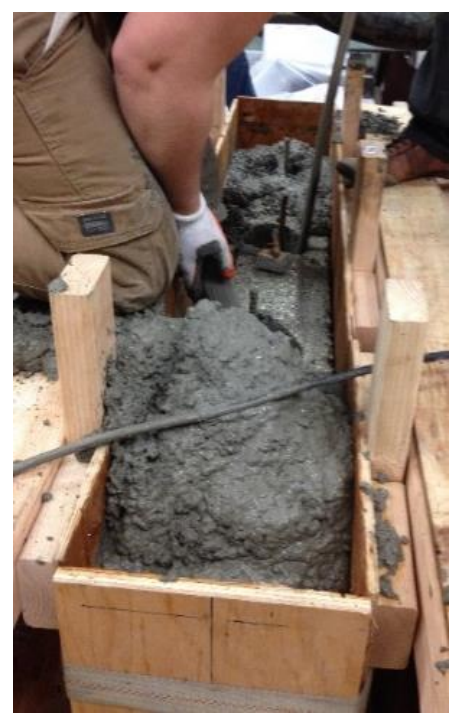

Concrete casting

Figure 3.3 Construction of the SGICF walls. 


\subsubsection{The New Pattern of SGICF Walls}

The second set of walls was constructed differently from the first set since the existing EPS blocks limited the spacing between the vertical cores to 16 in. on center. The primary goal was to build a wall that had the same distance between the outer vertical cores as of the first set of SGICF walls but with one less core. To illustrate, the newly suggested pattern of SGICF wall had three vertical cores and five horizontal cores as shown in Figure 3.4. Therefore, a new construction technique was used for this purpose to increase the spacing between the vertical cores to $24 \mathrm{in}$. on center. The new SGICF wall was constructed by using a 6 in. diameter Sonotube forms, which are large cardboard circular tubes that are designed to hold wet concrete in place until it hardens, and a 6 in. PVC drain T-connectors.

Unlike the EPS walls, the new pattern of SGICF walls had two reinforcement bars of \#4 in each vertical core and were placed with a clear spacing of 3.5 in.. Further, each wall had an integrated beam that was 6x10x60 in., which was cast on top of the wall to assist with load transfer from the steel load transfer beam along the top of the wall. The connection between the new pattern of SGICF walls and the top steel beam was secured by using J-bolts (7/8 in. diameter and $12 \mathrm{in.} \mathrm{long)} \mathrm{that} \mathrm{were} \mathrm{immersed} \mathrm{on} \mathrm{top} \mathrm{of} \mathrm{each}$ vertical core.

Figure 3.5 presents the newly adopted construction steps that were used to construct these walls. Similarly to the SGICF wall that was built out of EPS blocks, galvanized threaded rods were placed inside the 6 in. PVC T-connectors at the cores 
intersections to assist with the positioning of the horizontal rebar at the center of the cores and to mount the instrumentations.

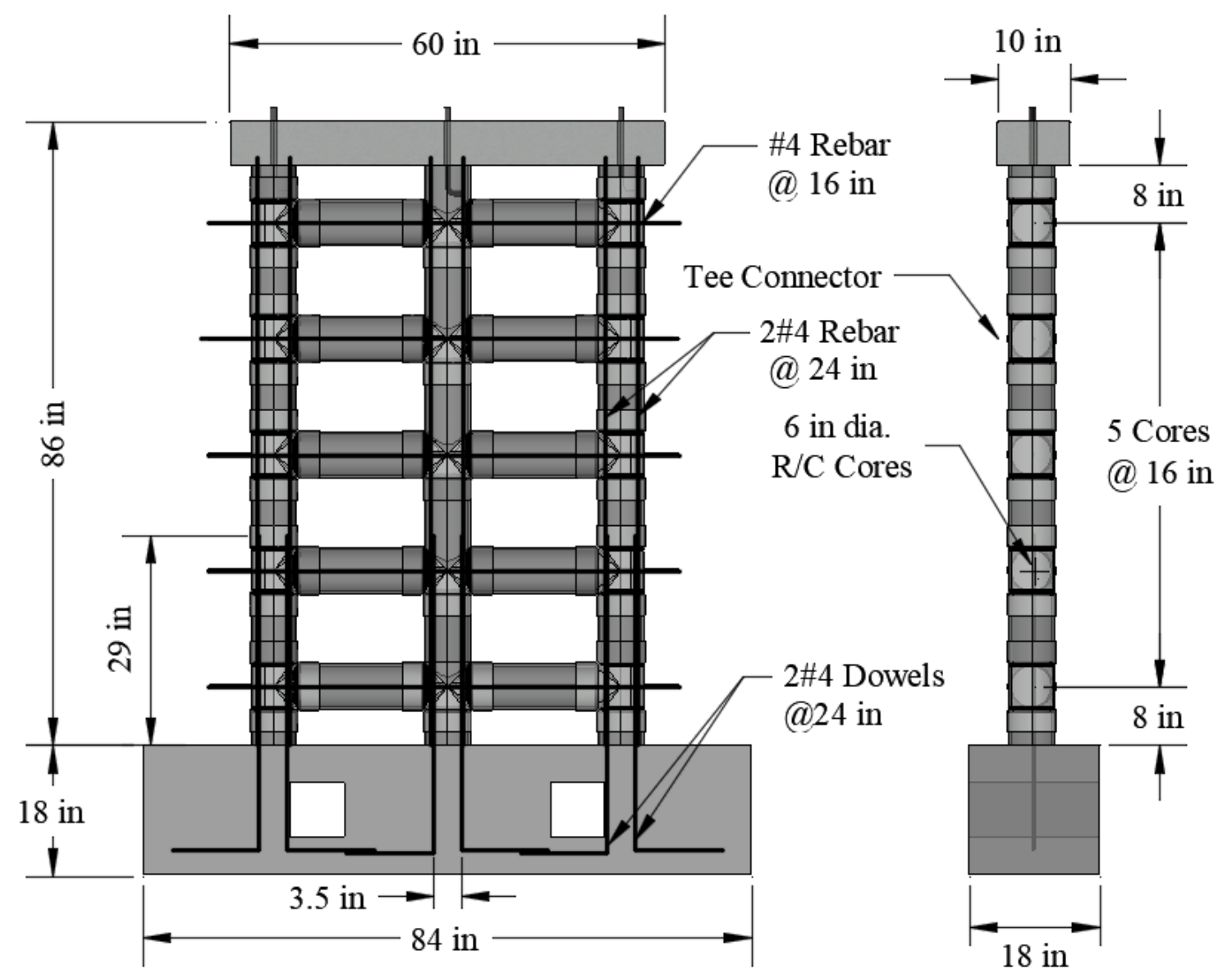

Figure 3.4 New pattern of SGICF wall geometry and reinforcement details. 


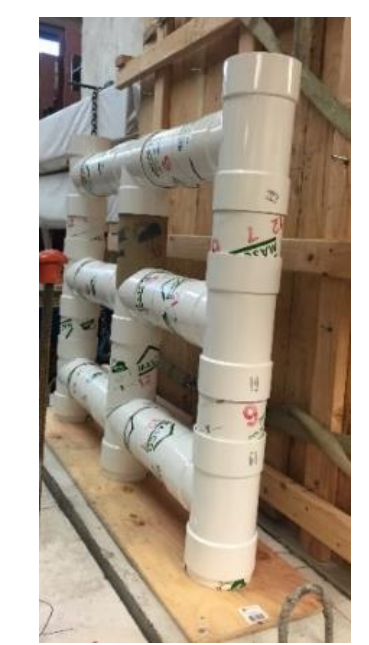

Components assembly

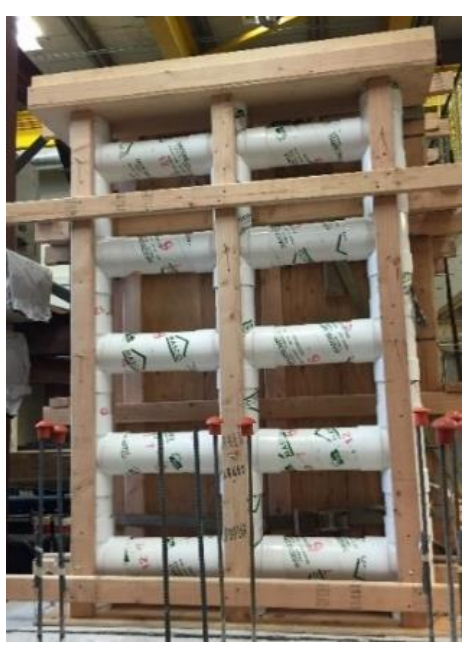

Wall forms

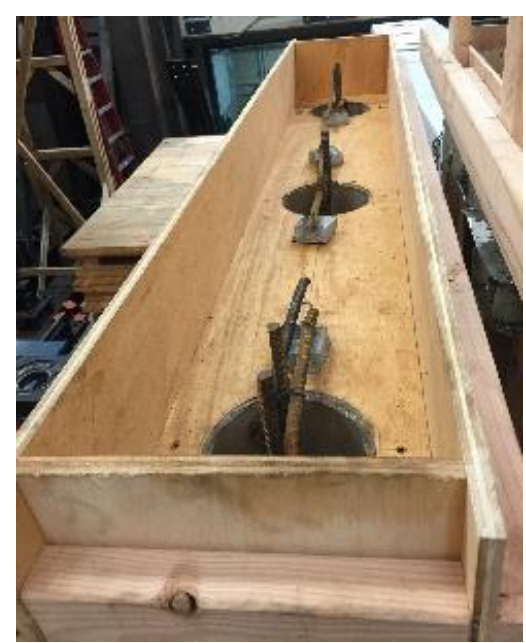

Bond beam forms

Figure 3.5 Construction of the new pattern of SGICF wall.

\subsection{Foundation Blocks}

The two sets of SGICF walls were constructed on top of strong reinforced concrete foundation blocks that have the same dimension of 7 x $1.5 \times 1.5 \mathrm{ft}$. Each block has two square openings ( $7.5 \times 7.5$ in.) to accommodate the square hollow structural section (HSS 7 x7x 3/8) steel beams that were used for clamping the wall to the shake table, as shown in Figure 3.6 \& Figure 3.8. The blocks were conservatively designed to limit the damage to only the SGICF walls. From each block, \# 4 dowels were designed according to section 25.5 of the ACI-318 (ACI 318-14, 2014) to be 29 in. that extended and aligned with the vertical core rebars. Figure 3.7 shows the construction of the foundation blocks. 
The longitudinal reinforcement was the same for the top and bottom of the foundation blocks and consisted of \#6 bars that were spaced 3 in. on center except for the coroner bars that were 2 in. on center. The transverse reinforcement consisted of \#4 hoops that were doubled-up throughout the foundation blocks except near the opening duct. Single \#4 hoops were used around the opening duct due to the limited spacing and to avoid rebar congestion. The concrete mix used for the foundation blocks was assigned for compressive strength of 3000 psi at 28 days and a slump of 6.5 in. The maximum nominal coarse aggregate size was $1 / 4-i n$. The concrete chute was used for pouring the concrete with a slump of 6.75 in. as shown in Figure 3.9.

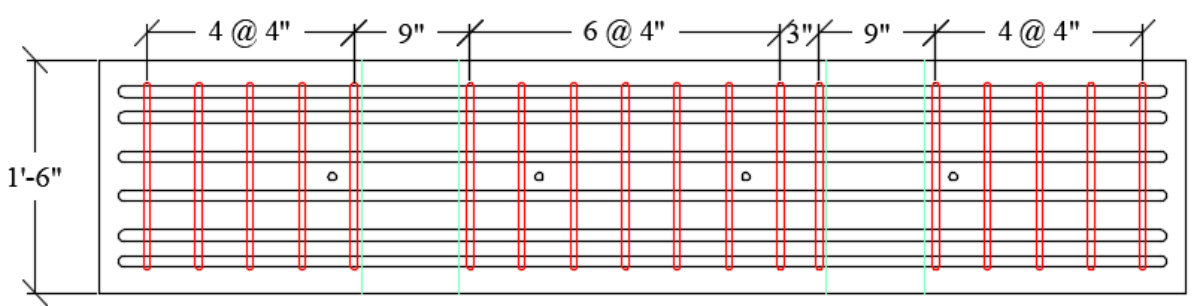

PLAN VIEW

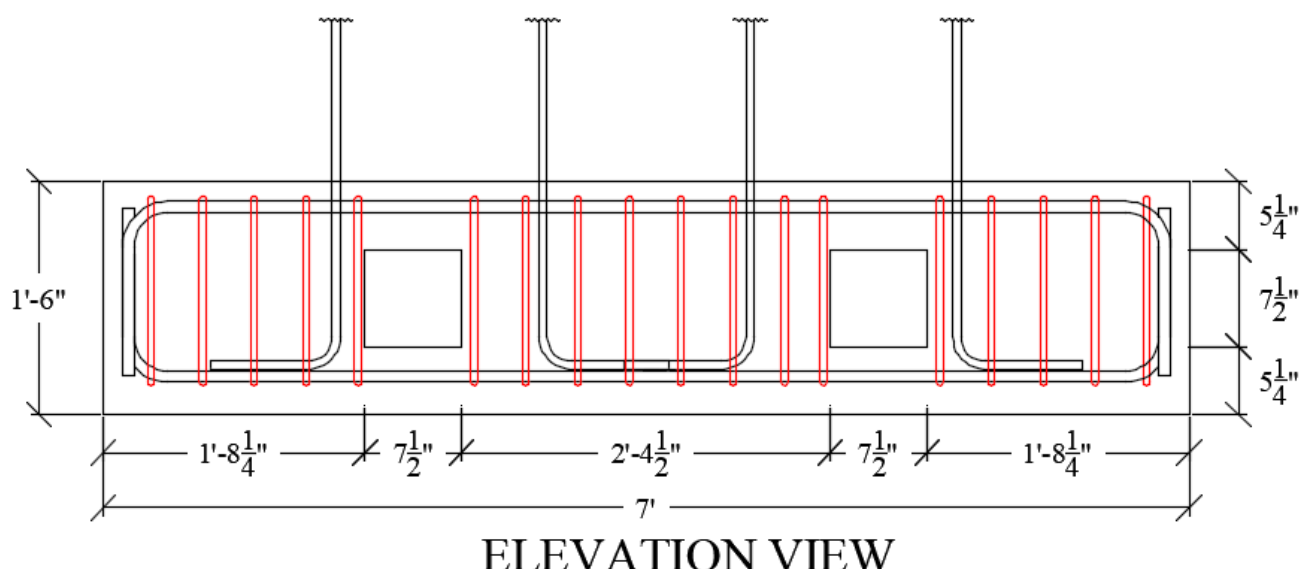

Figure 3.6 SGICF wall foundation block detail. 


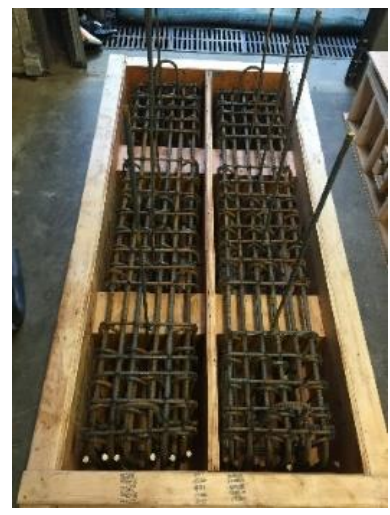

Forms and cages

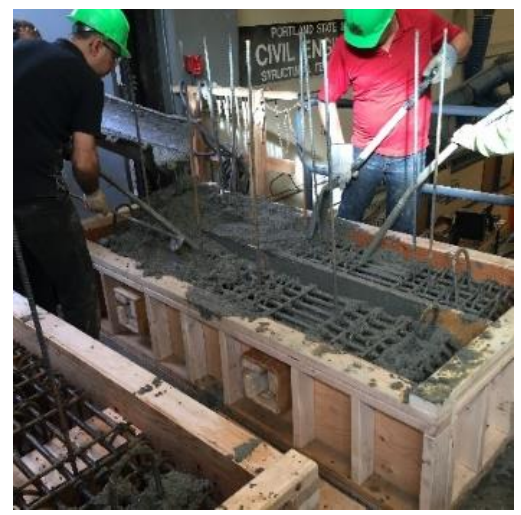

Concrete casting with vibration

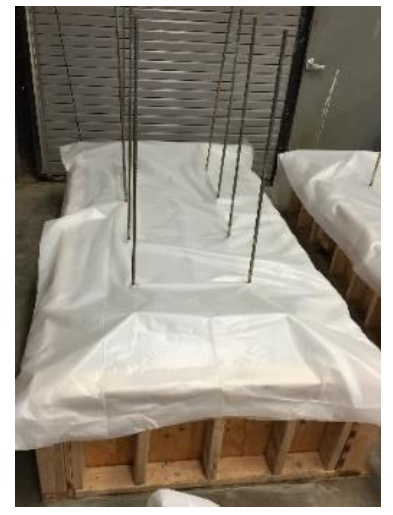

Concrete curing

Figure 3.7 Construction of SGICF wall foundation block.

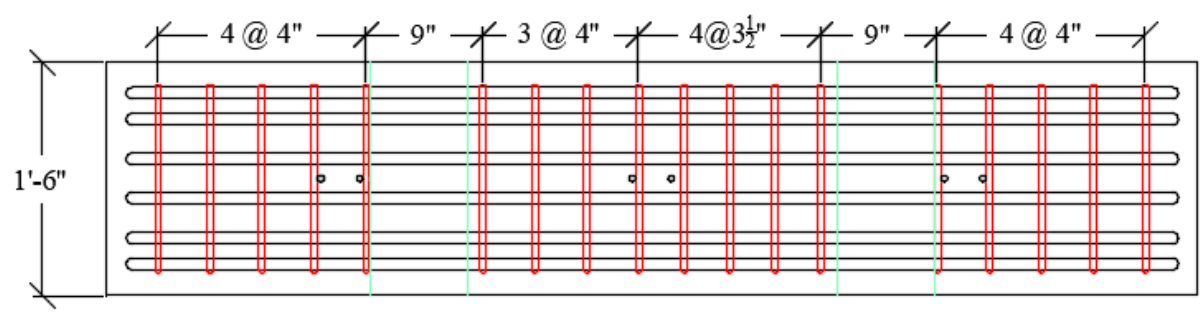

\section{PLAN VIEW}

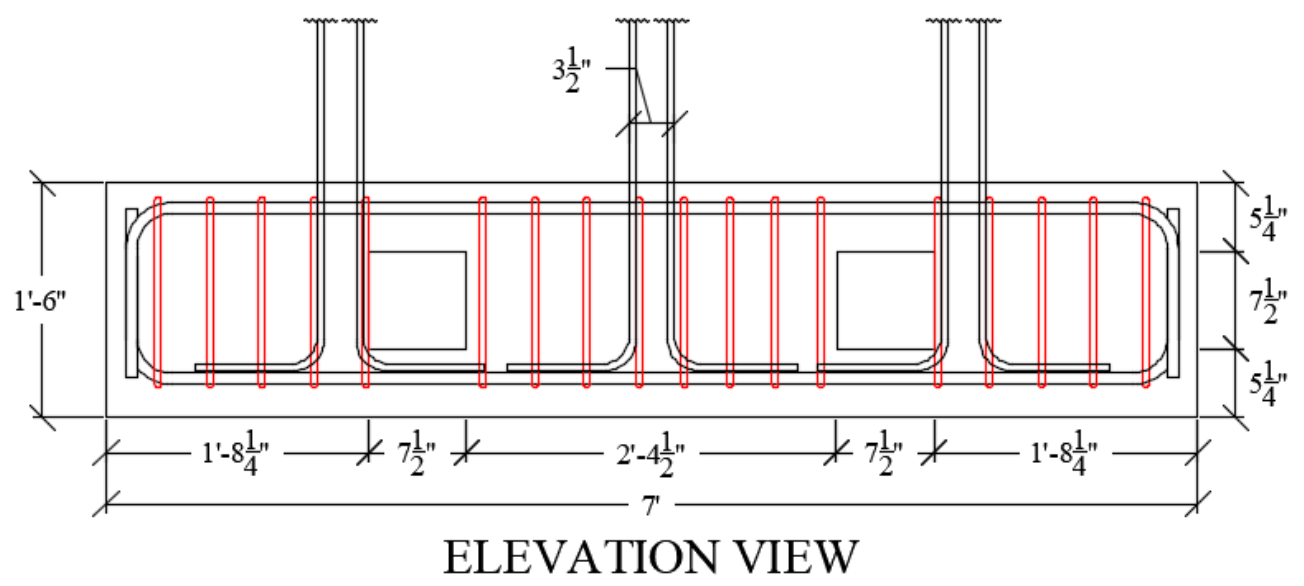

Figure 3.8 New pattern of SGICF wall foundation block detail. 


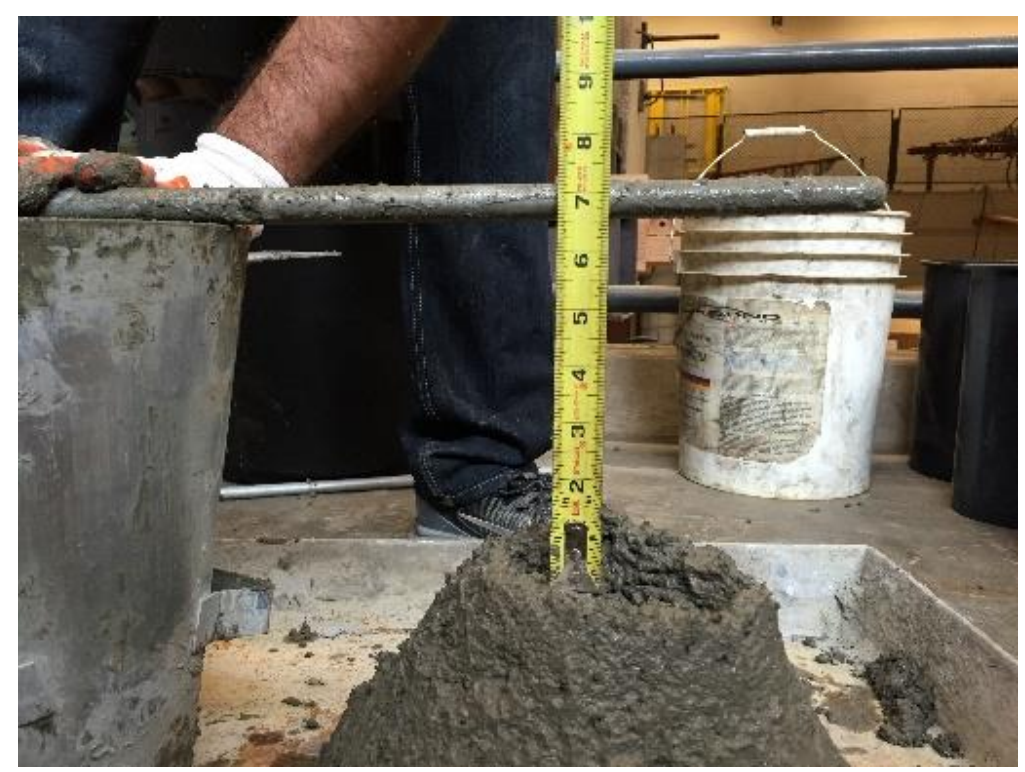

Figure 3.9 Slump test for the foundation blocks.

\subsection{Material Properties}

\subsubsection{Concrete}

Two types of concrete were used in this study: reinforced concrete and high tensile twisted micro steel fibers that were mixed with the reinforced concrete. The compressive strength of the concrete was determined according to the American Society for Standard Testing Materials (ASTM) International standard ASTM C 39/C 39M-10. For each type of concrete, one concrete cylinder (6 x 12 in.) was tested at seven and fourteen days after concrete placement. Later, two cylinders were tested at 28 days, and on the day of the wall testing. Compressive strength results for each wall are shown in 
Table 3-2. Figure 3.10 shows the compression machine with the specimen that was used to evaluate the compressive strength of the wall's concrete.

Table 3-2 Average compressive concrete strengths.

\begin{tabular}{|c|c|c|c|c|}
\hline Specimen & $f_{c}^{\prime}(\mathrm{psi})$ & 7-days $f_{c}^{\prime}(\mathrm{psi})$ & 14-days $f_{c}^{\prime}$ (psi) & 28-days $f_{c}^{\prime}$ (psi) \\
\hline W-RC & 6208 & \multirow{2}{*}{3069} & \multirow{2}{*}{4131} & \multirow{2}{*}{4966} \\
\hline G-RC & 5917 & & & \\
\hline W-FB & 5515 & \multirow{2}{*}{2778} & \multirow{2}{*}{3801} & \multirow{2}{*}{4214} \\
\hline G-FB & 5148 & & & \\
\hline
\end{tabular}

The concrete mix design for all walls specified 3/8 in. coarse aggregate, clean sand, 8.5 in. slump, and a 28 -days strength of 3000 psi. The concrete ready mix was provided by Ross Island Sand \& Gravel of Portland, OR. From Table 3-2, it is obvious that the steel fiber-concrete compressive strength was less by $12 \%$. This reduction in the compressive strength of the fiber-concrete resulted from the extra water added to the concrete mix to maintain the required slump of 8.5 in. that was reduced due to the addition of the twisted micro steel bar to the mix. 


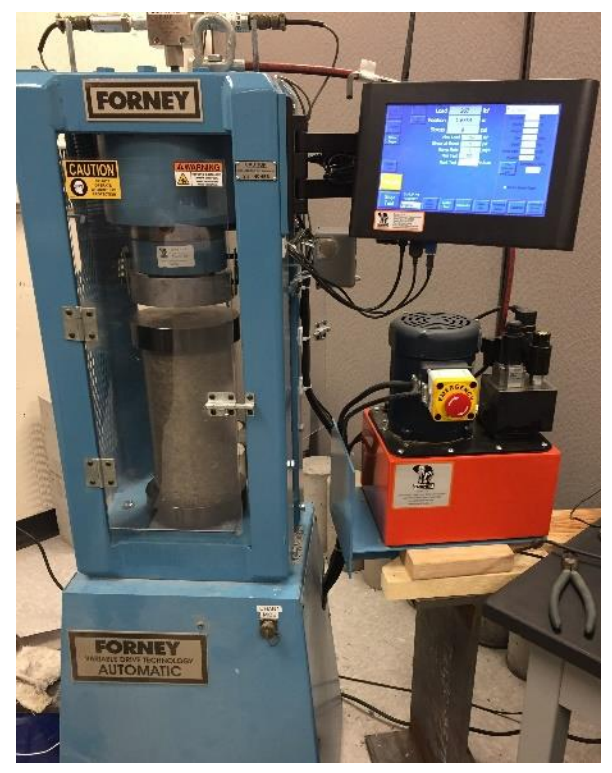

Figure 3.10 Apparatus used to determine the compressive strength of the concrete.

\subsubsection{Steel}

The mechanical properties of the reinforcing steel bars were measured in an experimental tensile test using one of the deformed $0.5 \mathrm{in}$. diameter steel bar used for both horizontal and vertical cores reinforcements. The test was conducted according to the ASTM A370-17 by IMR Test Labs of Portland, OR. Figure 3.11 shows the apparatus used for the tensile test of the reinforcement bar. The measured yield strength was 64.5 ksi, and the ultimate tensile strength was 95 ksi. Figure 3.12 shows the stress-strain curve of the tested rebar. 


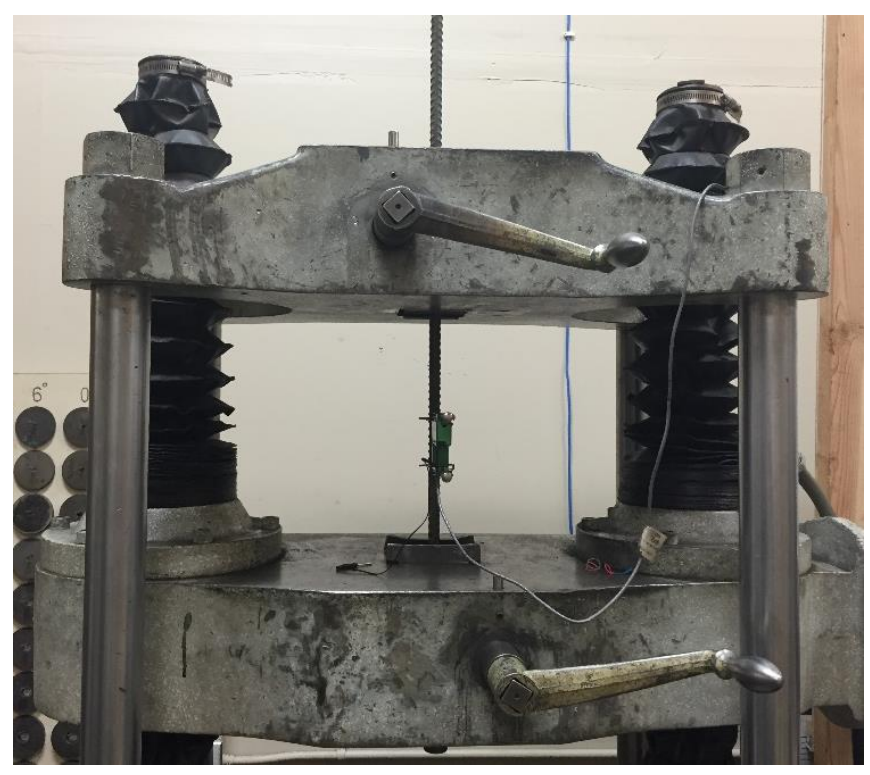

Figure 3.11 Apparatus used to determine steel reinforcement tensile strength.

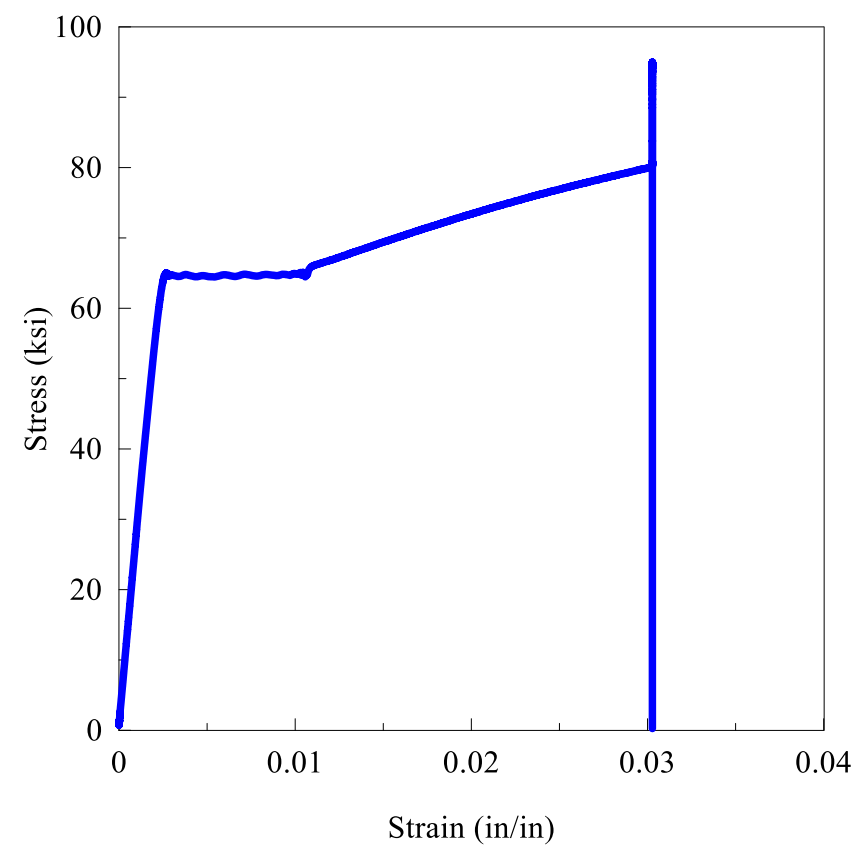

Figure 3.12 Stress-strain curve for \#4 steel reinforcement bar. 


\subsection{Test Rig}

Shake table tests require inertial loads to study the dynamic effects on the performance of SGICF walls. A variety of approaches are available to assess the dynamic behavior of structural elements (Carrillo, Gonzalez, \& Llano, 2012), in which each approach has its advantages and drawbacks. The mass rig that was utilized for this study consisted of a steel column pin-connected to the shake table. The inertia mass consisted of four concrete blocks that were attached to the steel column with high strength posttension rods.

A safety steel frame was installed to prevent the inertia mass from falling and damaging the shake table in the event of wall failure. Two guiding casters were provided to the web of each longitudinal beam to prevent out-of-plane motion of the inertial mass. The mass-rig was then connected to the load transfer steel beam with an axially rigid link that was pinned at both ends to provide an axially rigid link without imposing any additional rotations onto the test specimen. The load transfer beam was fastened to the top of the SGICF wall using J-bolts that were cast to the top of the wall. Out-of-plane bracings were attached to the load transfer beam at two locations to guide the wall while not providing any additional resistance to the movement in the plane of the wall. Figure 3.13 shows the mass rig system with the SGICF wall specimen.

The steel load beam was consisted of a W16x45 section that was 98.38 in. long with a 1.5 in. thick plate welded to the back of the beam. The thick plate was used to secure the top steel beam by placing two 2 -in. threaded rods that were used to provide a 
slip critical connecting between the load transfer beam and the top of the wall as shown in Figure 3.14. Five slotted holes of 7/8 x1-7/8 in. were drilled at the center of the W16x45 steel section to assure the alignment of the SGICF wall with the inertia mass link as shown in Figure 3.15.

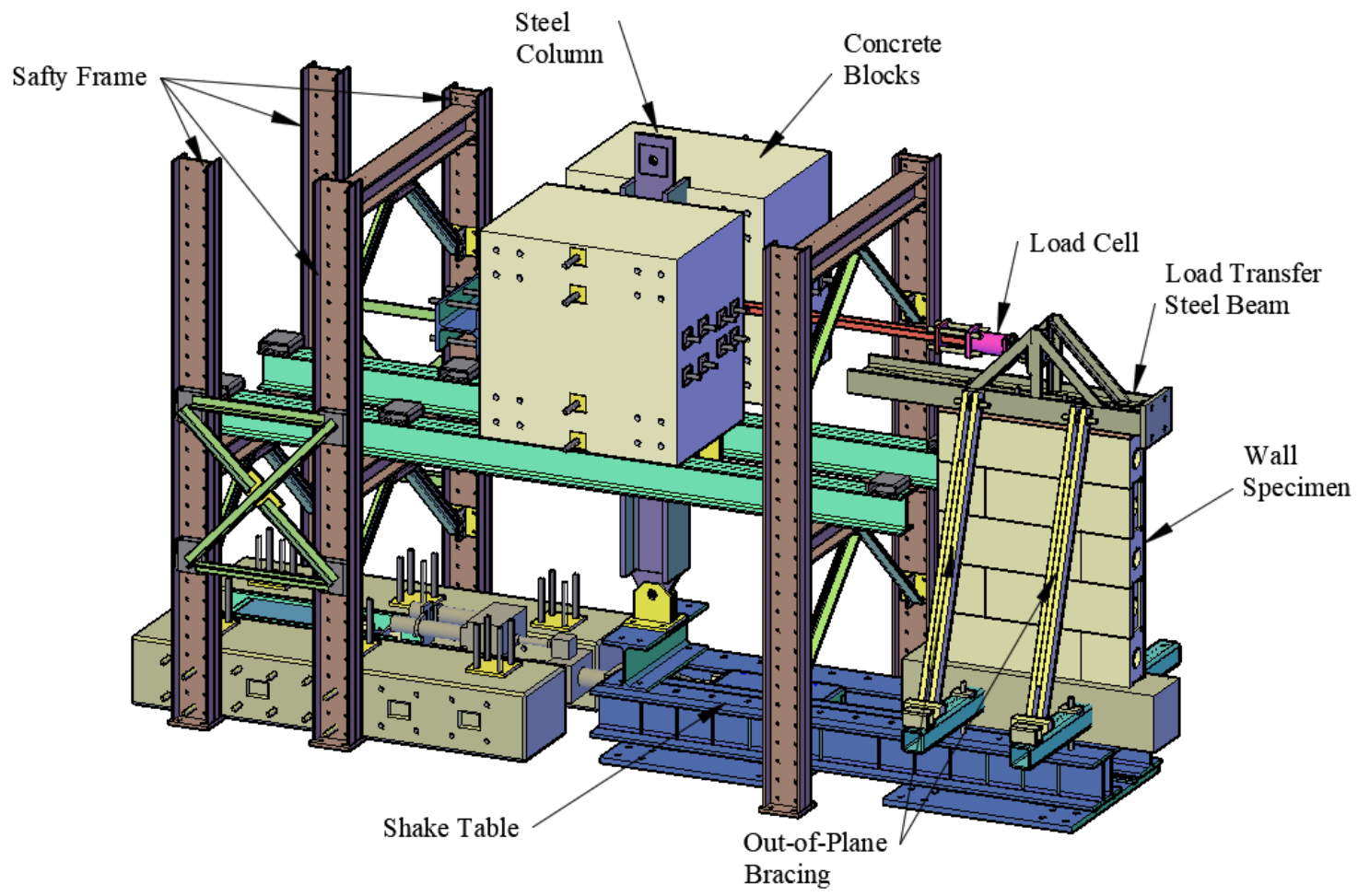

Figure 3.13Mass rig system with test specimen 


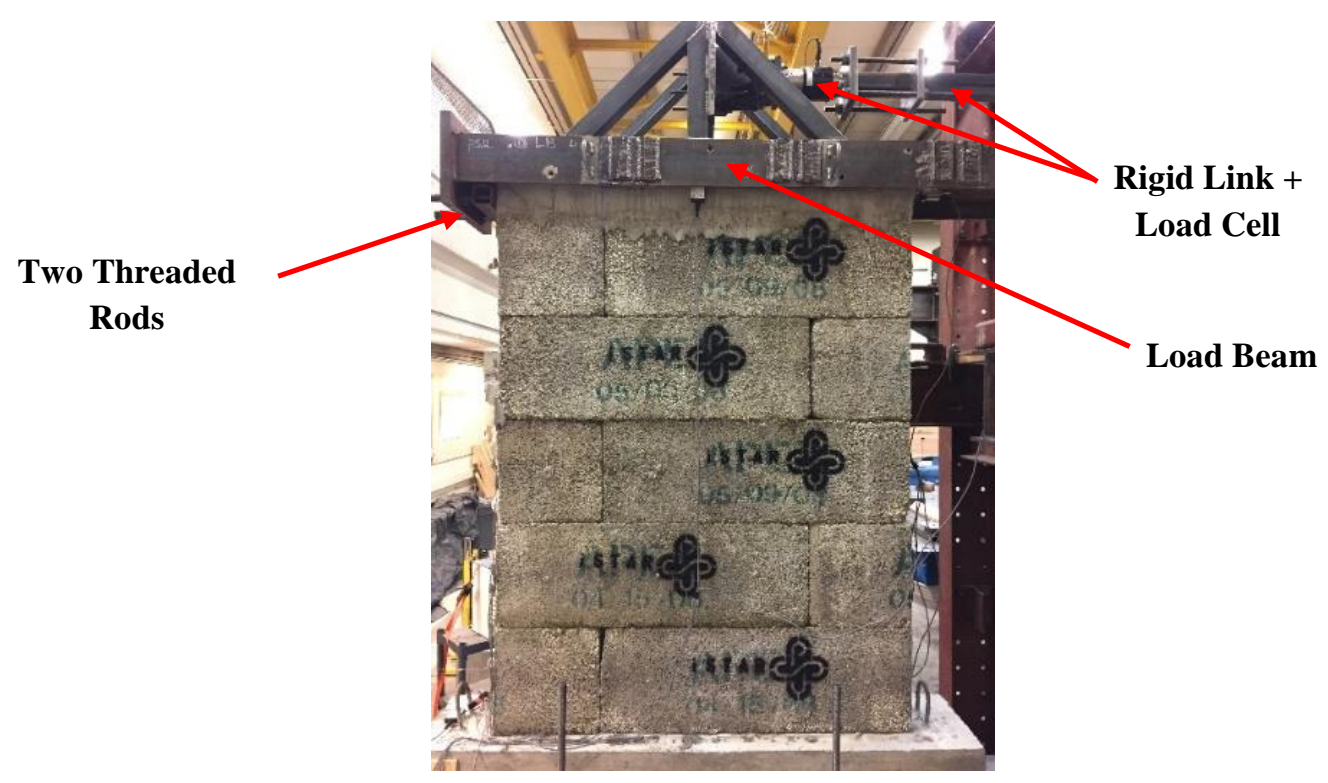

Figure 3.14 SGICF wall with the load beam and load cell.
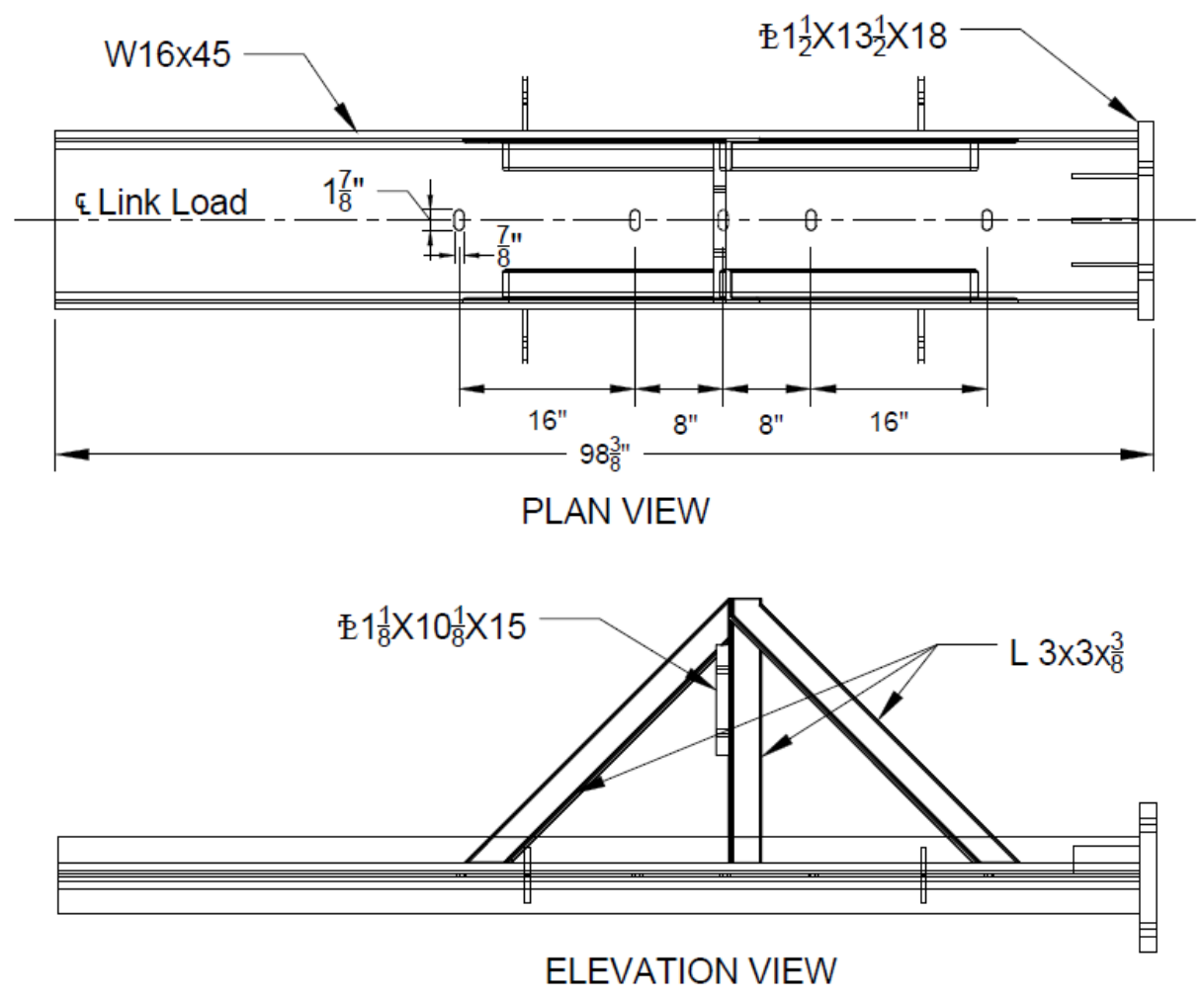

Figure 3.15 Details of load beam 


\subsection{Ground Motion Selection}

Imperial Valley earthquake motion was selected as the input for the shake table test, which recorded during the M6.5 event with peak ground acceleration (PGA) of 0.24 $\mathrm{g}$ in the $\mathrm{x}$-direction. Figure 3.16 shows the selected ground motion time history and the associated response spectrum assuming the typical 5\% damping. A free vibration was conducted before and after each test to obtain the dynamic properties of each wall, such as period and damping ratio. The input ground motion acceleration was incrementally increased for each specimen in an effort to observe the linear and nonlinear behavior of the walls. The scaled ground motion started at 0.3 times the record to capture what the elastic response would be, then was increased to 0.5 of the full record. Each subsequent motion was then raised by a factor of 0.25 amplitude.

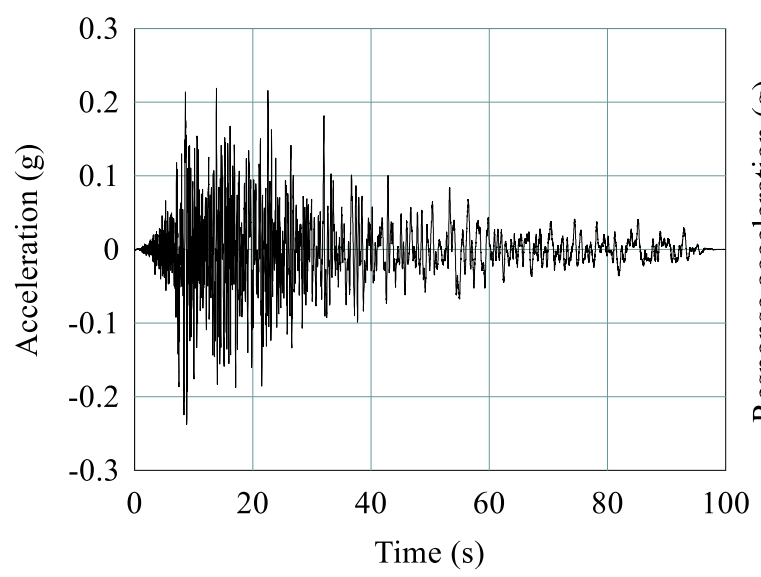

(a)

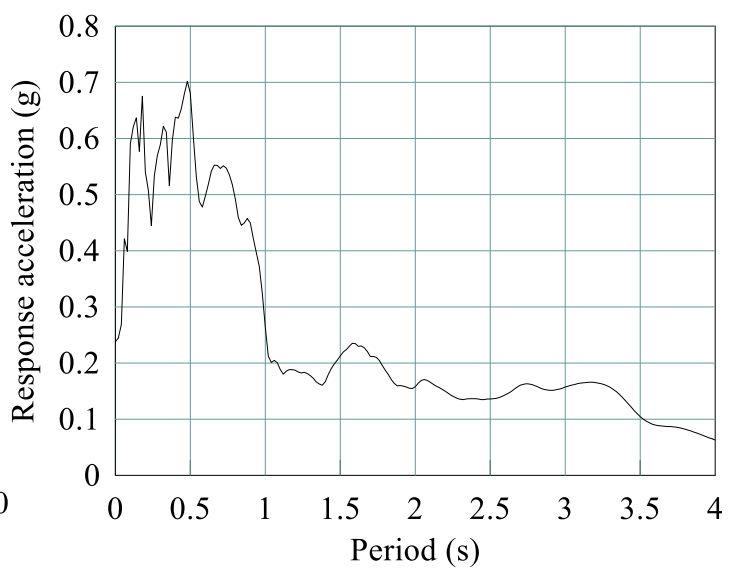

(b)

Figure 3.16 Selected ground acceleration: (a) time history, (b) response spectrum. 


\subsection{Instrumentations}

In order to analyze the behavior of the SGICF walls subjected to the time history loadings, the specimens were instrumented internally and externally. The internal instrumentations included ten strain gauges, and the external instrumentations consisted of accelerometers, linear variable displacement transducers (LVDTs), string pots, and load cell.

The lateral load strength of each wall was measured by using a MTS load cell with a maximum force capacity of 55000 pounds that was attached to a swivel and rigid links as shown in Figure 3.13 and Figure 3.14. The strain gauges were used to measure the strain at specific points where the plastic hinges expected in the specimens. For WRC and W-FB walls, four strain gauges (SG 1-4) were mounted in each dowel of the footings at a distance of 1.0 in from the footing face as shown in Figure 3.17. 


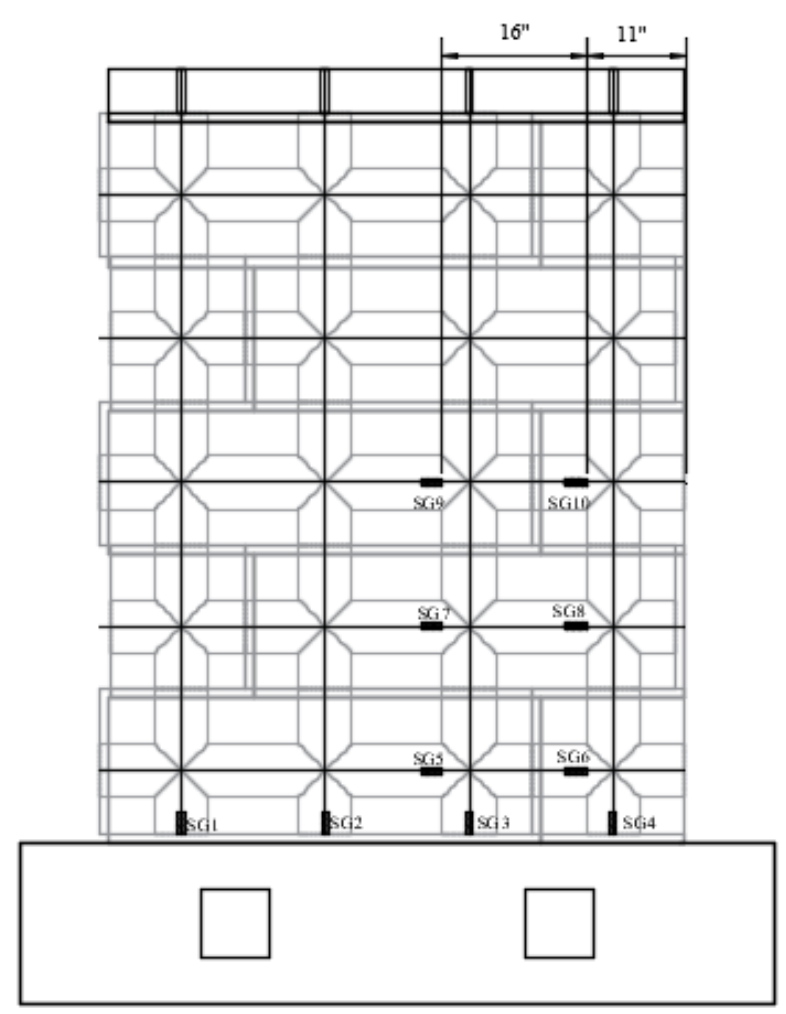

Distribution of strain gauges

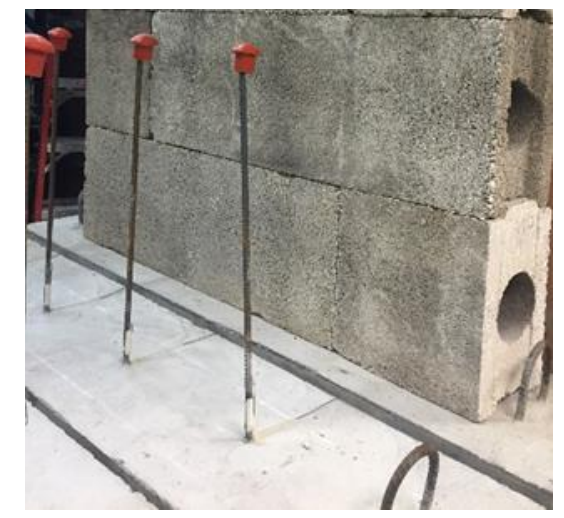

Dowels strain gauges

Figure 3.17 Location of strain gauges for W-RC and W-FB specimens.

A total of 25 LVDTs were used to measure a variety of displacements that the wall, foundation block, and the load beam were expected to experience. For clarity, LVDTs (1-8) were used to measure walls curvature at different wall's heights.

Meanwhile, LVDTs (9-20) were placed diagonally on the wall to measure the shear deformation between vertical cores. Four LVDTs were placed on the foundation block to verify that no slipping (LVDT 21 and LVDT 22) or uplift (LVDT 23 and LVDT 24) was occurring between the block and the shake table. One LVDT (25) was used to measure the relative displacement between the load beam and the top of the wall. 
Five string potentiometers were used at each level of the horizontal cores (SP 1-5) to measure in-plane wall deflections. Four accelerometers were installed at different locations on the setup: top of the wall, top of the masses, top of the footing, and the middle of the rigid link as shown in Figure 3.19. Figure 3.18 shows the location of the external instrumentations used for W-RC- and W-FB walls.

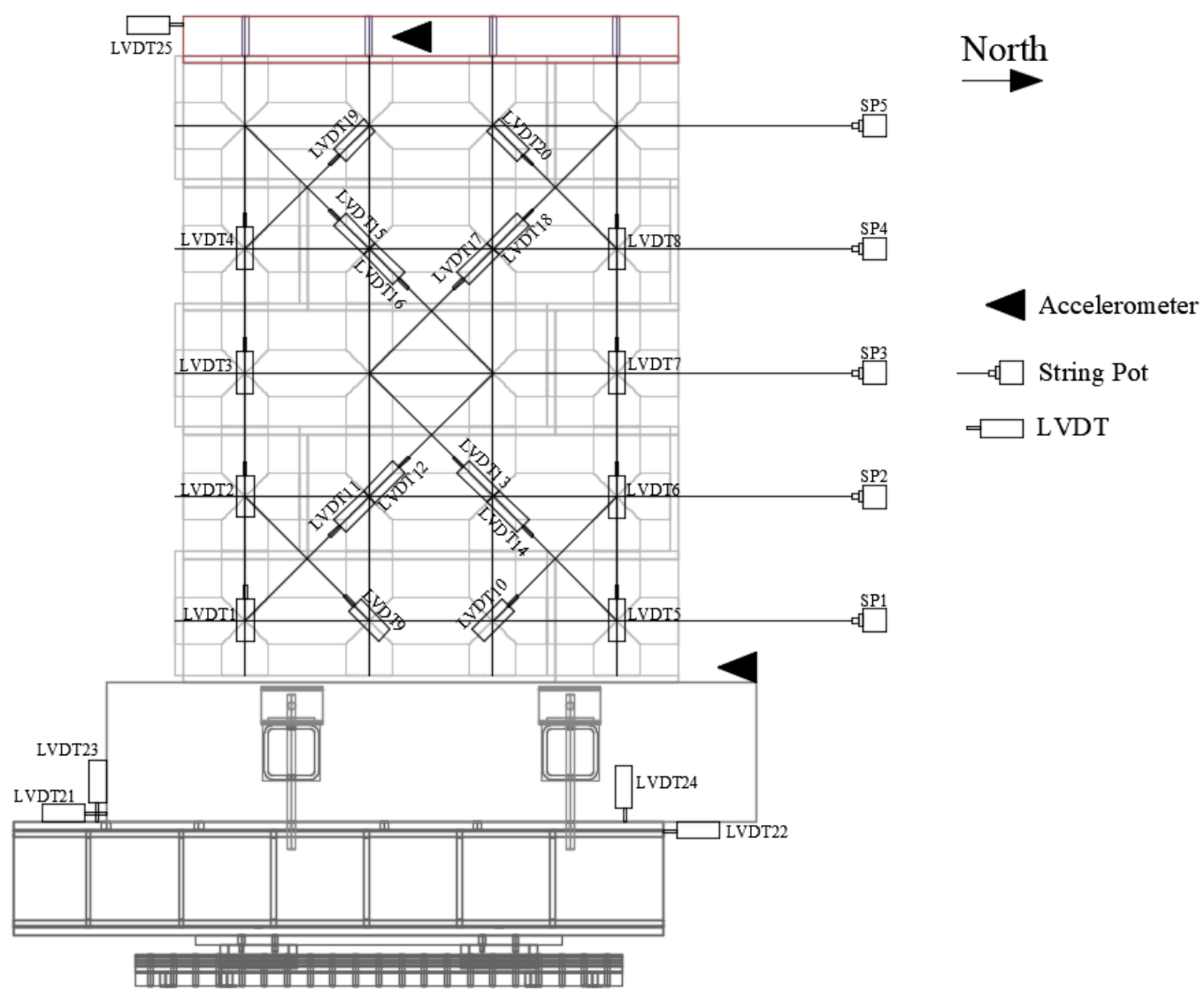

Figure 3.18 Location of LVDTs, string pots, and accelerometers for W-RC and W-FB specimens. 


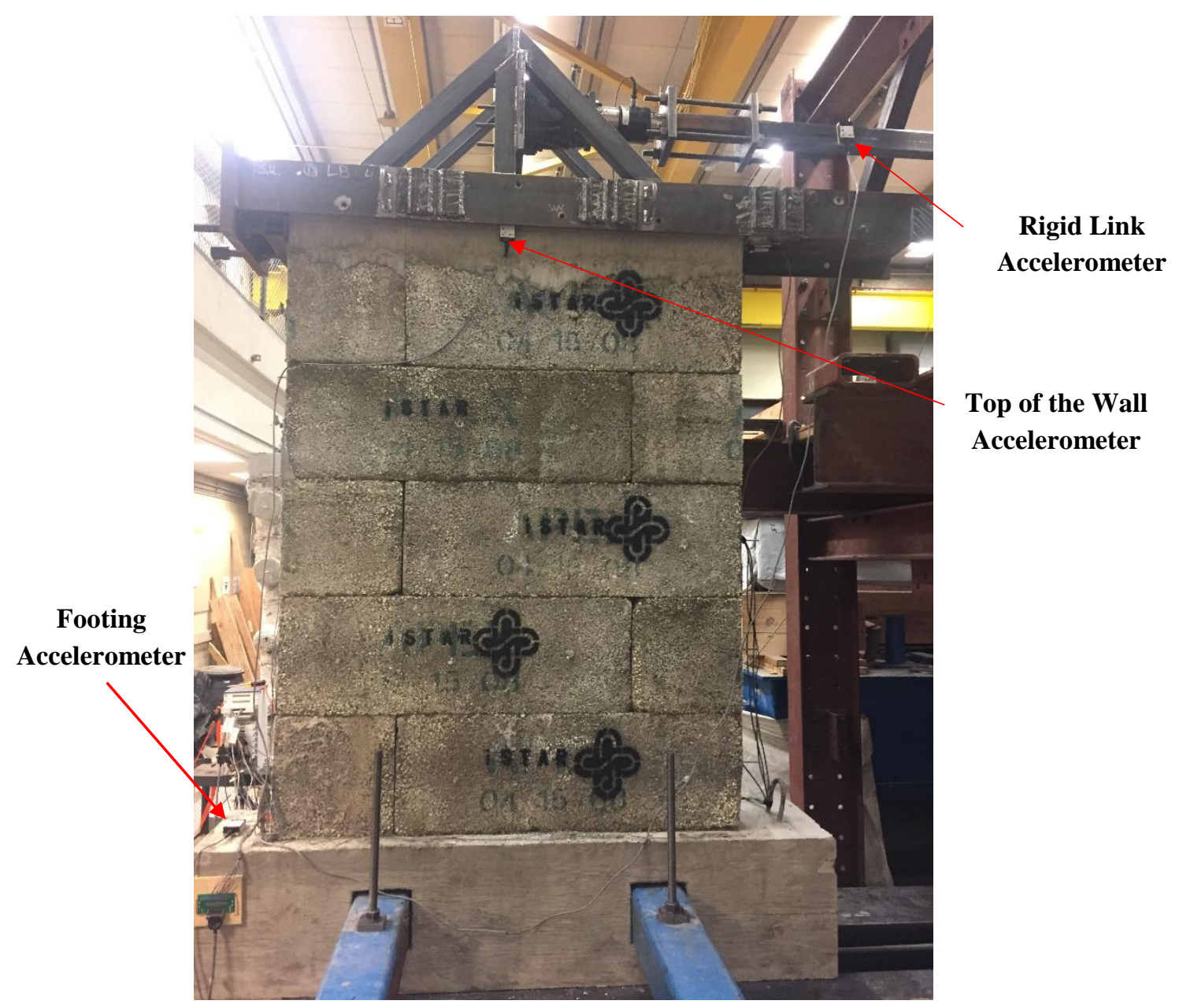

Figure 3.19 Location of accelerometers on the specimen.

The internal and external instrumentations of the G-RC and W-FB walls were slightly different than those of the walls that built out of the ICF blocks. Accelerometers and string pots locations were the same. Dowels strain gauges were placed on the outer vertical cores rebar as shown in Figure 3.20.

A total of 17 LVDTs were used in G-RC and G-FB walls. LVDTs (1-4) were used to measure walls curvature at different wall's heights, and LVDTs (5-12) were placed diagonally on the wall to measure the shear deformation between vertical cores. Four 38 
LVDTs were positioned on the foundation block to verify that no slipping (LVDT 13 and LVDT 14) or uplift (LVDT 15 and LVDT 16) was occurring between the block and the shake table. One LVDT (17) was used to measure the relative displacement between the load beam and the top of the wall. Figure 3.21 shows the location of the external instrumentations used for G-RC- and G-FB walls.

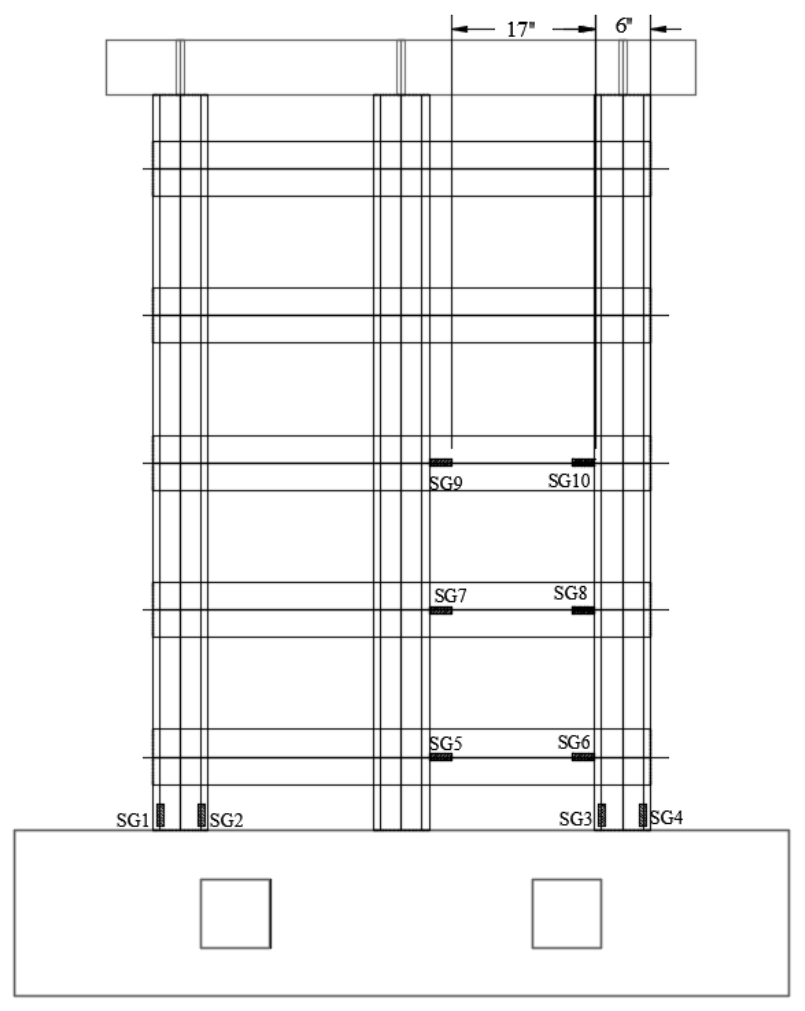

Distribution of strain gauges

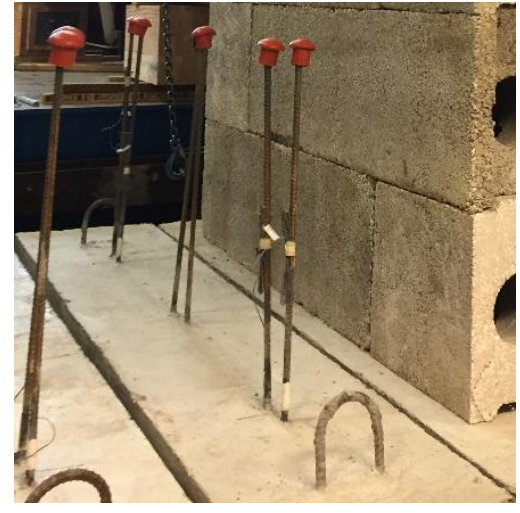

Dowels strain gauges

Figure 3.20 Location of strain gauges for GRC and G-FB specimens. 


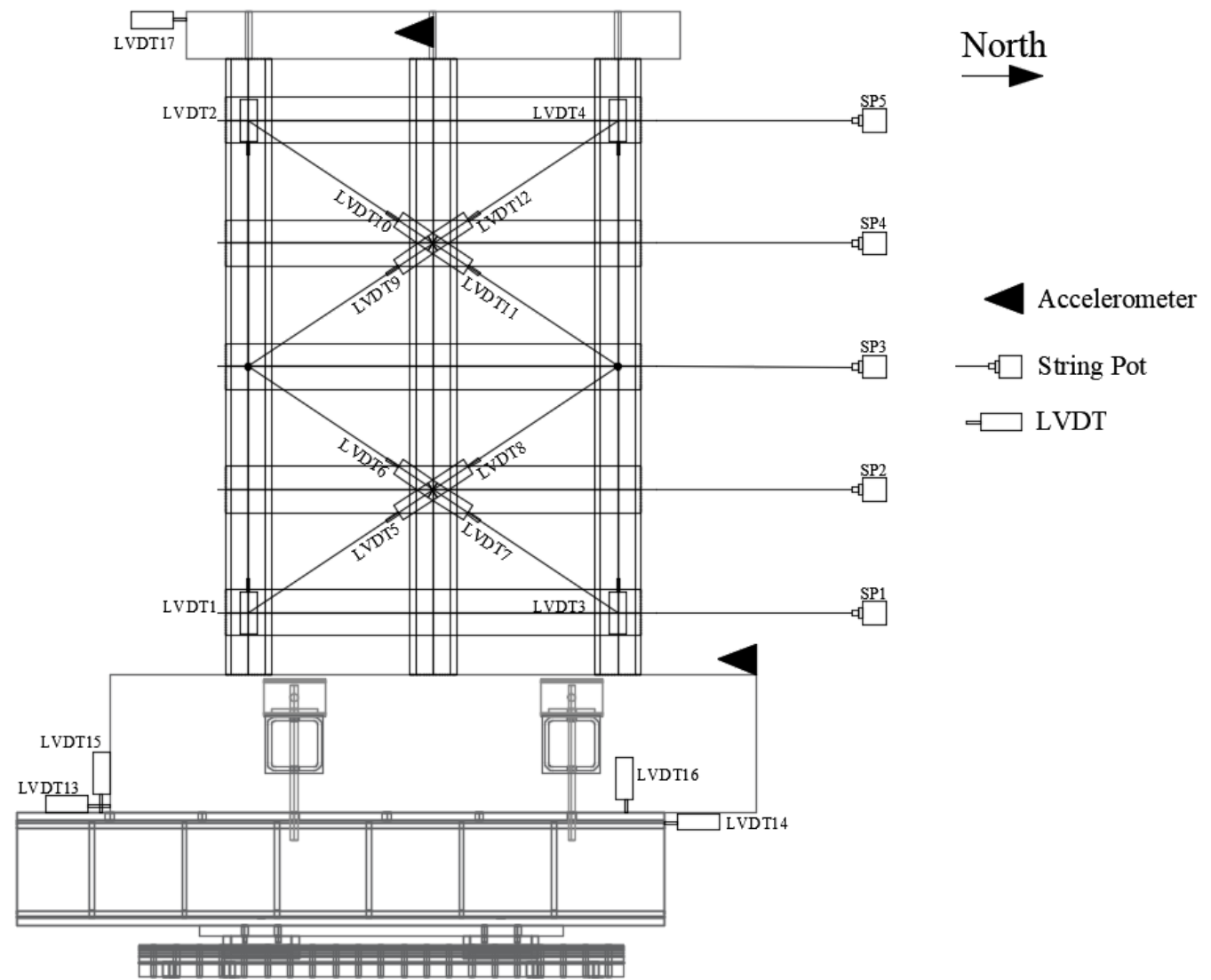

Figure 3.21 Location of LVDTs, string pots, and accelerometers for G-RC and G-FB specimens.

\subsection{Test Results and Observations}

Each wall was subjected to several scaled ground motions and brought to failure, either by specimen collapse or through monitoring the reduction in the lateral strength. When the reduction in the lateral load resisted by the wall is equal or more than $20 \%$ of the recorded peak load, a decision to terminate the test was taken. The maximum drift capacity of each wall was considered as the maximum lateral displacement measured at the end of the last applied scaled ground motion. 


\subsubsection{SGICF Walls}

The failure modes for W-RC and W-FB walls were the same during the low scale factors of the ground motion (SFGM). Observation of cracks and damage was limited due to the presence of the ICF blocks. During the high scaled ground motions, damages to the ICF blocks were more visible, especially on W-FB wall that had the steel fiber-concrete cores.

\subsubsection{W-RC}

Figure 3.22 shows failure modes at the end of the scaled ground motion ranged from 0.3-1.0. Visual observation of cracks and damage during the low scale factors of the ground motion was limited due to the presence of the ICF blocks. By their presence, the blocks obscured the cores and did not necessarily exhibit small cracks due to the deformable nature of the polystyrene.

Before and after each ground motion, a free vibration test was performed to obtain the dynamic properties of the specimen, such as natural period, damping ratio, and stiffness. The damping ratio $\xi$ was calculated using the decrement logarithmic approach (Chopra, 2011), according to the following equation:

$$
\ln \frac{u_{i}}{u_{i+1}}=\frac{2 \cdot \pi \cdot \xi}{\sqrt{1-\xi^{2}}}
$$

Where, $u_{i}$ and $u_{i+1}$ are the peak values of displacement, force, or acceleration at the first and $\mathrm{i}^{\text {th }}$ successive cycle, respectively. 
Analyses of the free vibration test resulted in a period of 0.28 second and a damping ratio of $0.92 \%$. After the $\mathrm{W}-\mathrm{RC}$ was subjected to ground motion scaled to 0.3 , a stiff response within the elastic range of walls' strength was observed as shown in Figure 3.23a. When ground motion intensity was increased, the base of the wall uplifted from the foundation by the yielding of the vertical core rebar, especially at the outer cores. After reaching the full-scale ground motion, i.e., with amplitude scale factor of 1.0 , the maximum drift reached was $1.33 \%$ with the maximum lateral strength of 16.0 kips. Figure 3.23 shows the degradation in specimen strength.

The degradation was also observed from the measured dynamic properties obtained after application of each ground motion. The natural period of vibration degraded from 0.28 second to 0.51 second after completion of the full amplitude ground motion, i.e., ground motion amplification of 1.0. The damping ratio increased from $0.92 \%$ to $3.1 \%$. Despite the strength degradation, there was not any noticeable exterior damage observed as shown in Figure 3.22d. The failure modes shown in Figure 3.22 did not show any obvious damages to the EPS blocks at the end of the application of the ground motion amplification of 1.0 .

Subsequent ground motion scaled to 1.25 of the earthquake record resulted in the complete collapse of the specimen. As shown in Figure 3.24, three vertical rebars fractured and the entire wall collapsed by lifting off the foundation and tilting. The maximum drift before the collapse was $2.6 \%$ before terminating the test, with the maximum lateral strength of 18.2 kips as shown in Figure 3.25. 


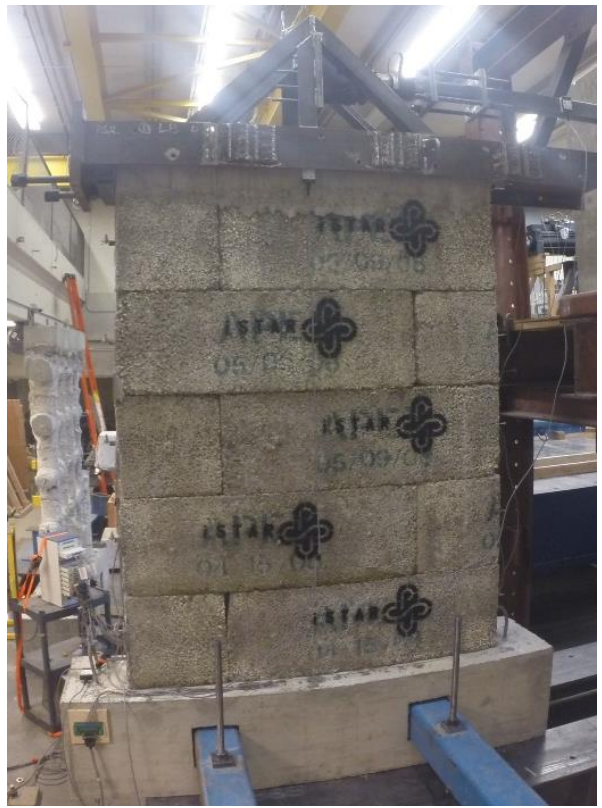

(a)

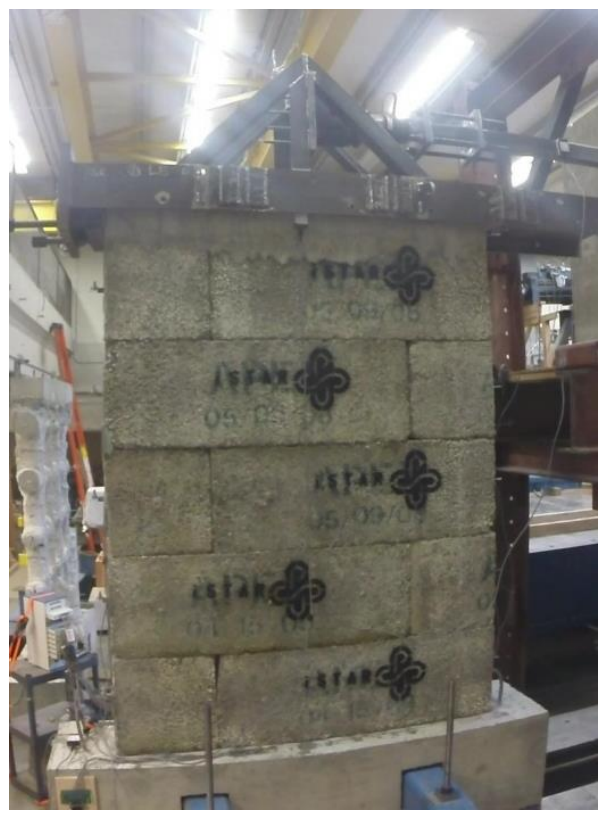

(c)

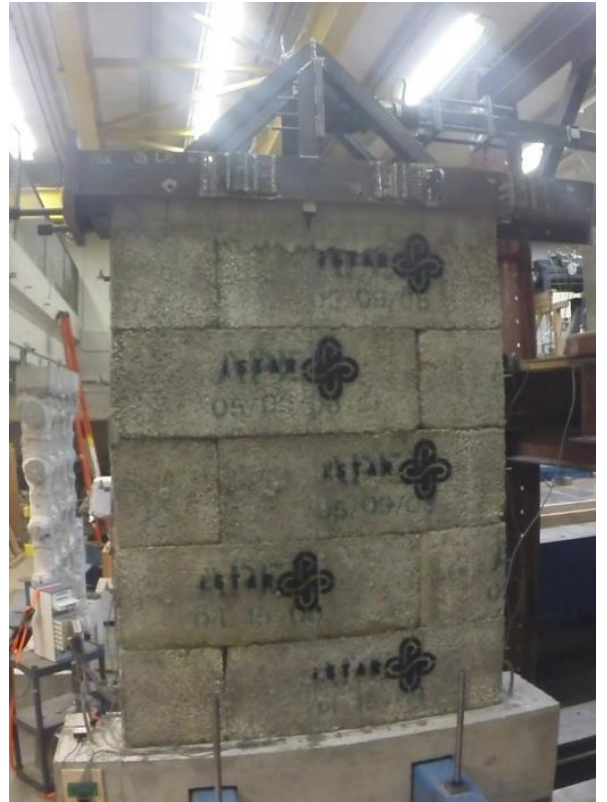

(b)

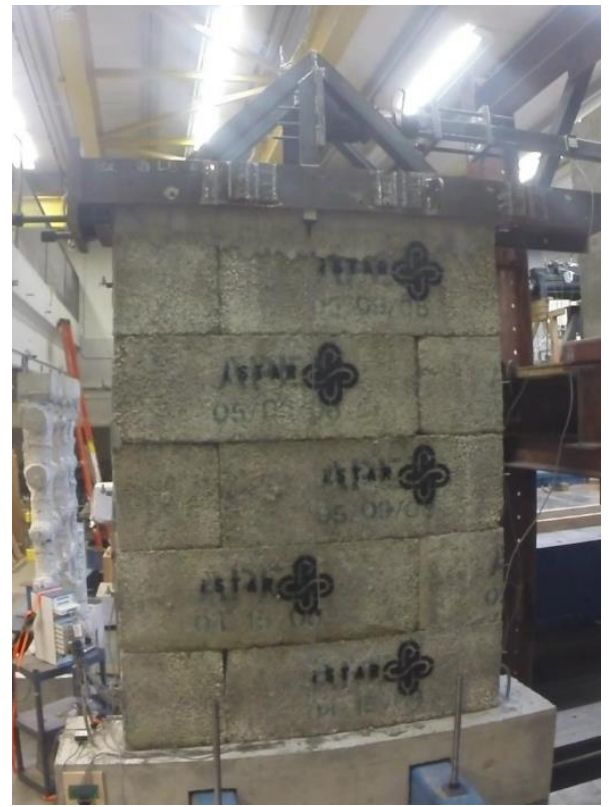

(d)

Figure 3.22 Failure mode of W-RC: (a) SFGM-0.3, (b) SFGM-0.5, (c) SFGM-0.75, (d) SFGM-1.0. 


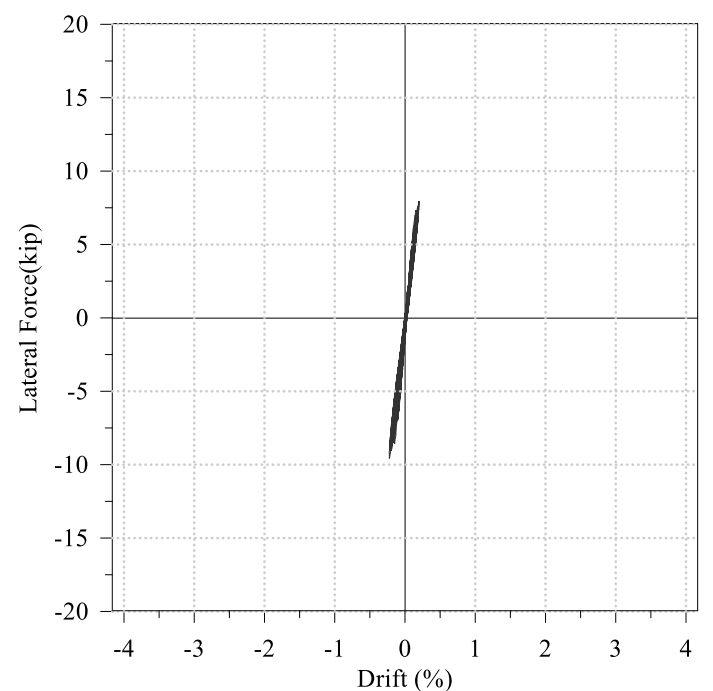

(a)

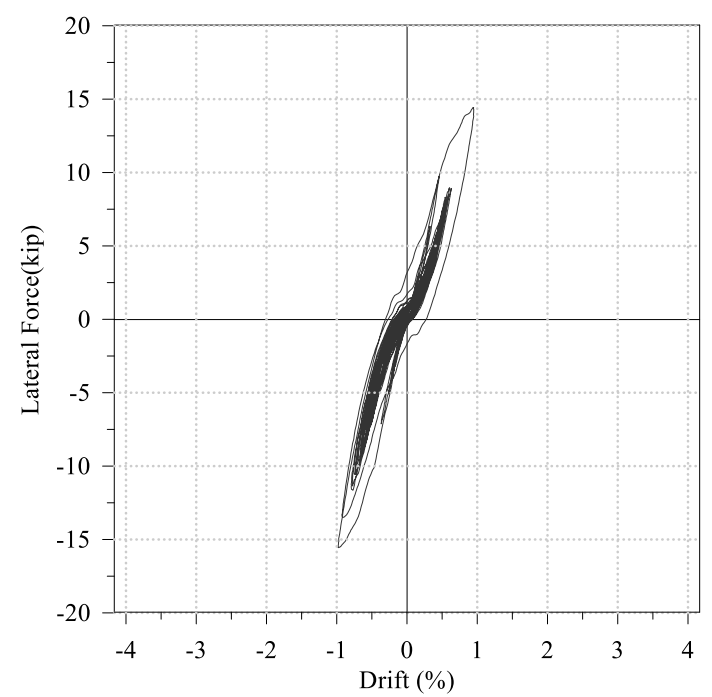

(c)

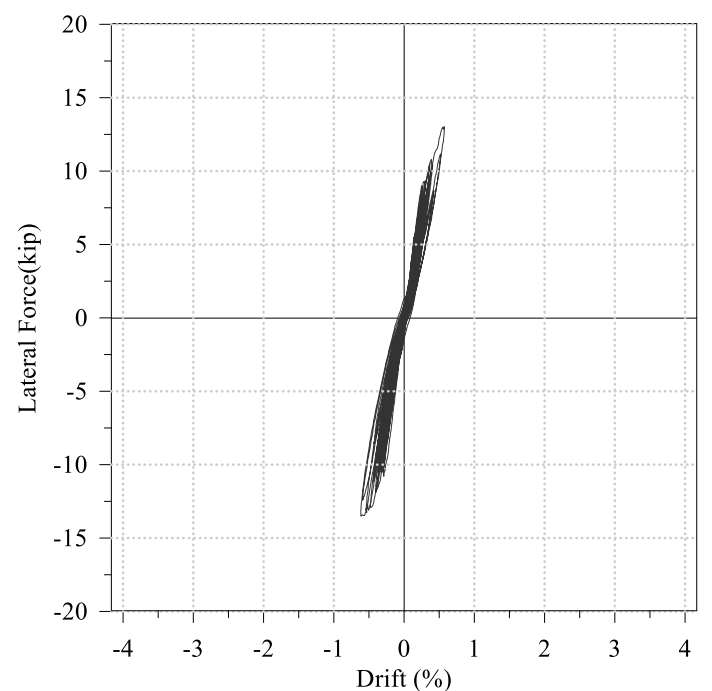

(b)

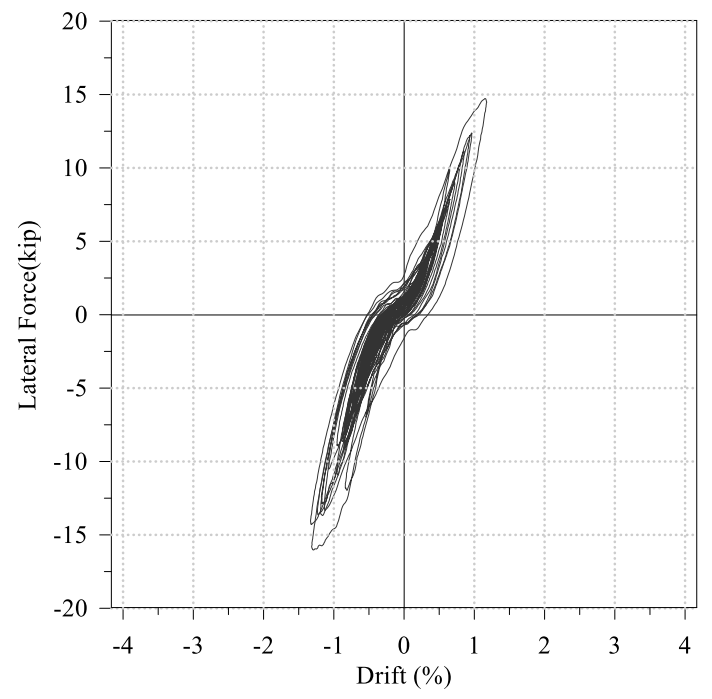

(d)

Figure 3.23 Earthquake response for W-RC: (a) SFGM-0.3, (b) SFGM-0.5, (c) SFGM-0.75, (d) SFGM-1.0. 


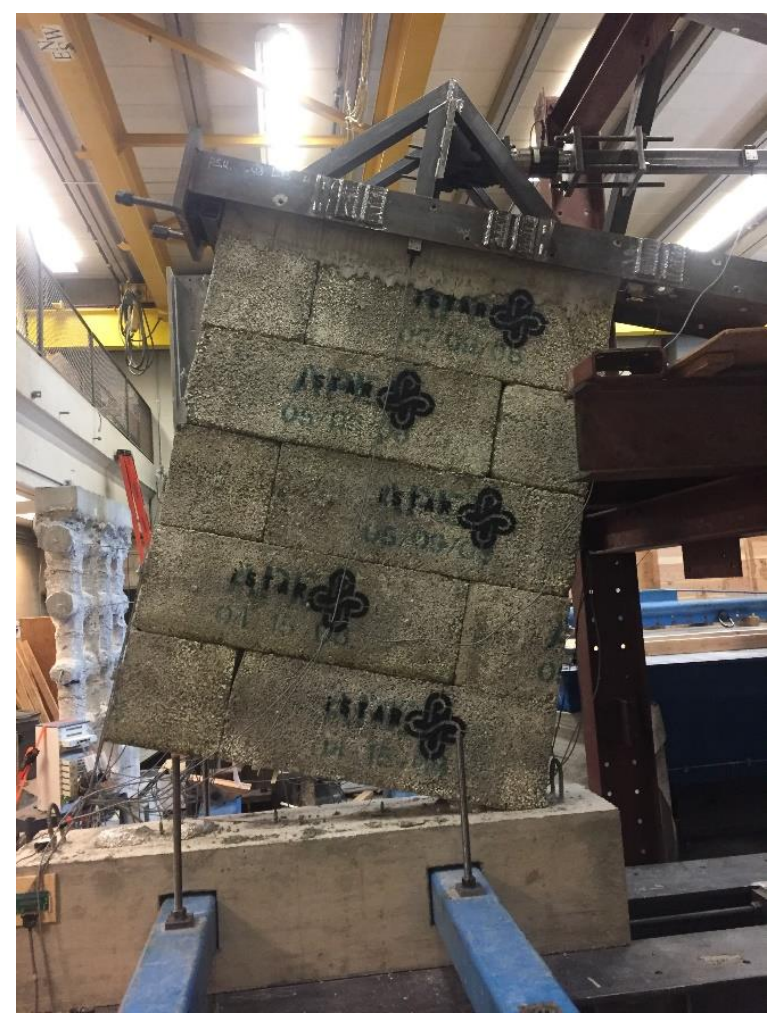

Figure 3.24 Collapse of W-RC at SFGM-1.25.

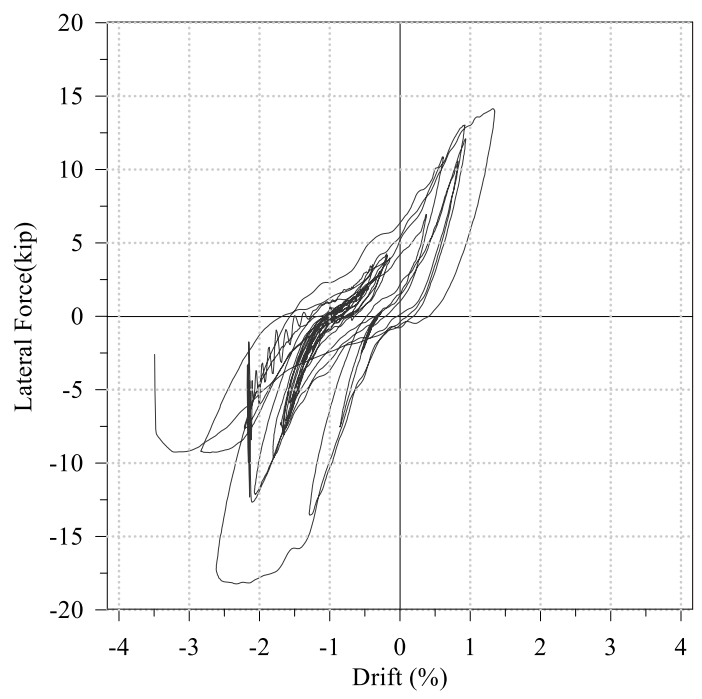

Figure 3.25 Hysteresis response of W-RC to 1.25 scaled ground motion. 


\subsubsection{W-FB}

Figure 3.26 shows the failure modes at the end of the scaled ground motion ranged from $0.3-1.0$. The initial natural period of vibration was 0.3 second and had a damping ratio of $1.0 \%$ as recorded from the free vibration. After the application of the ground motion scaled to 0.3 , a less stiff response was observed in the W-FB wall compared to the $\mathrm{W}-\mathrm{RC}$ wall, with a drift percent of $0.37 \%$ as shown in Figure $3.27 \mathrm{a}$. At the end of the ground motion scaled to 0.75 , the lateral strength was 12.9 kips, and the drift reached $1.57 \%$ as shown in Figure 3.27c. During this level of scaled ground motion, noticeable relative deformations were observed between the first and the third level of the horizontal cores as measured from the base of the wall. When the wall was subjected to full-scale ground motion, cracks in the EPS blocks themselves were observed as highlighted in Figure 3.26d and Figure 3.28.

The natural period of vibration degraded from 0.3 second to 0.57 second after completion of the ground motion scaled to 1.0, and the damping ratio was increased from $1.0 \%$ to $3.0 \%$. The maximum lateral strength was 14 kips and drift percent of $3.0 \%$ at the end of the test as shown in Figure 3.27d. Strength degradation of the specimen from 14 to 10.4 kips resulted in the termination of the experiment because the wall exceeded degradation to below $0.8 \mathrm{Vmax}$ (maximum lateral strength). Examination of the failure zone was conducted by stripping away the ICF blocks within the highlighted zone. Even though no clumping of steel fibers was observed, removing the ICF blocks revealed lack of consolidation of concrete within this zone as shown in Figure 3.28. 


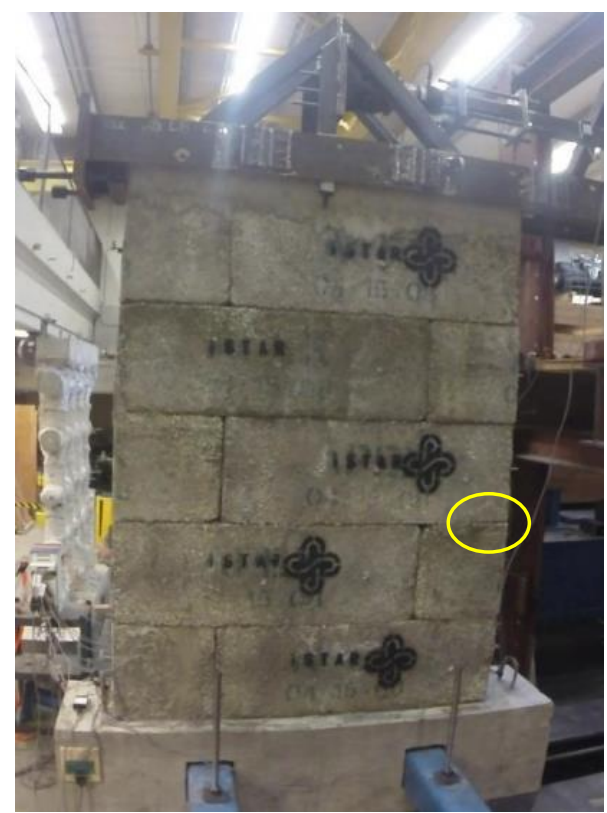

(a)

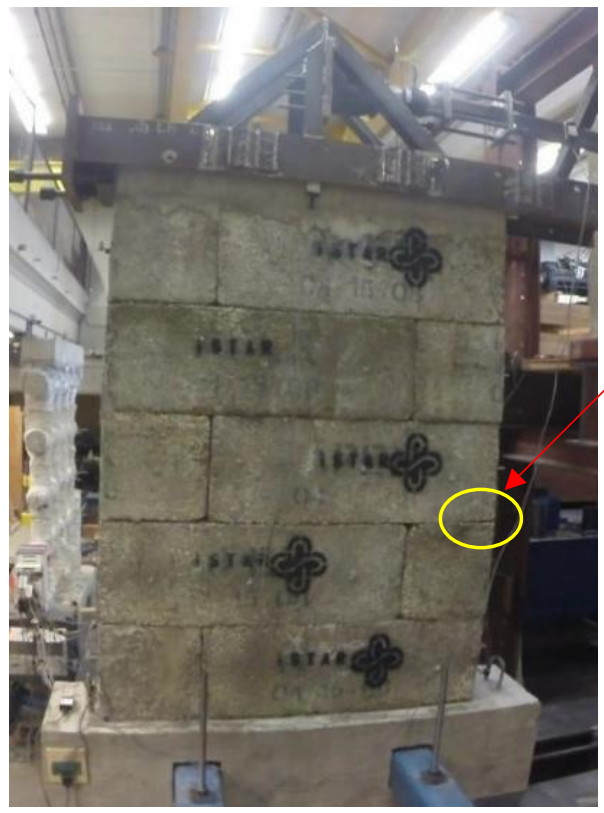

(c)

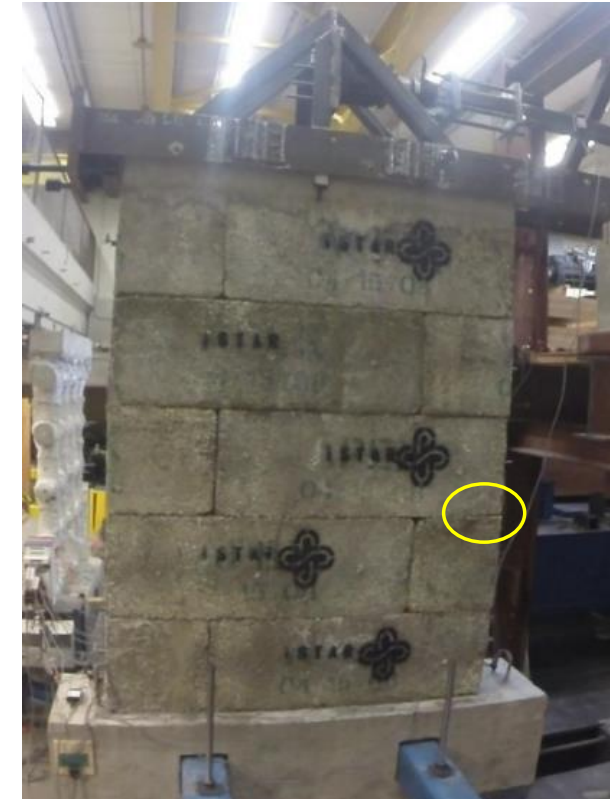

(b)

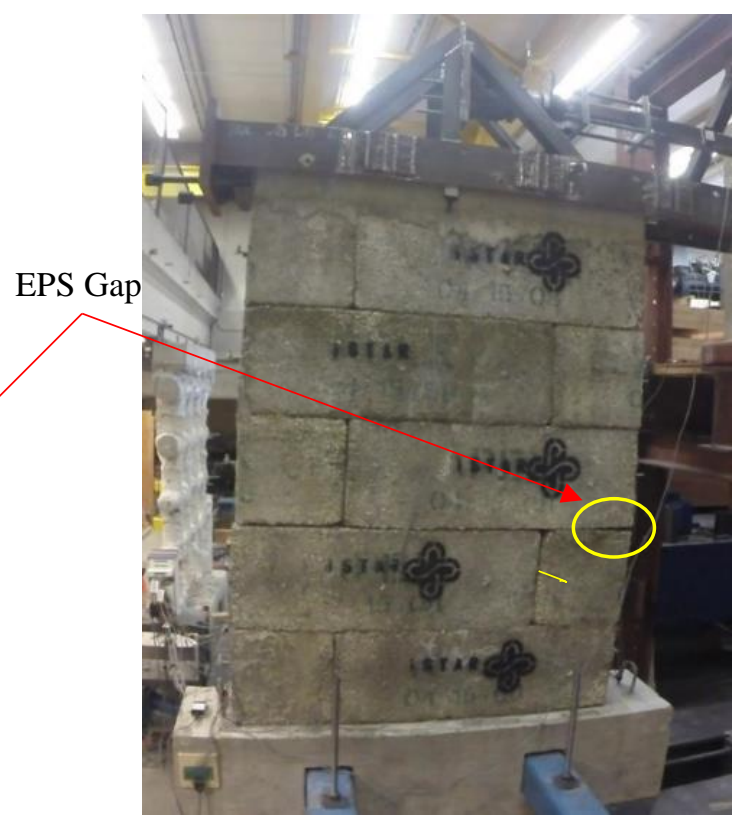

(d)

Figure 3.26 Failure mode of W-FB: (a) SFGM-0.3, (b) SFGM-0.5, (c) SFGM-0.75, (d) SFGM-1.0. 


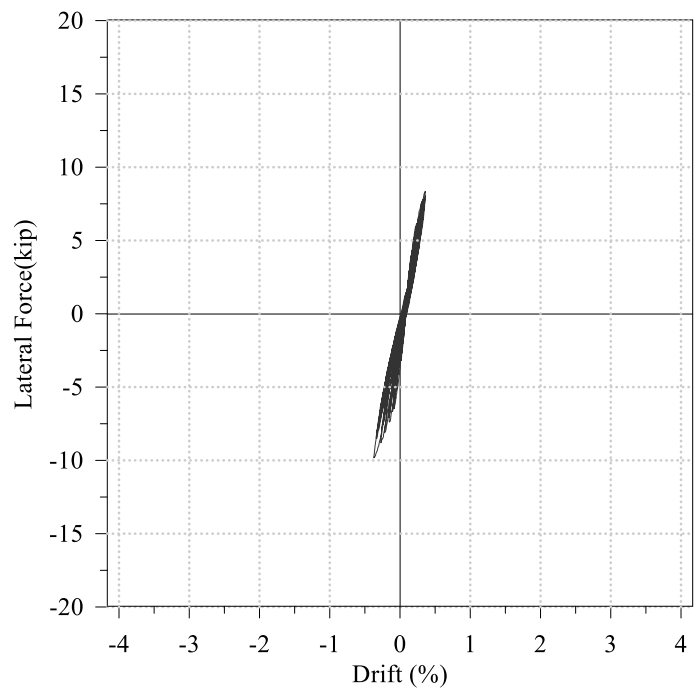

(a)

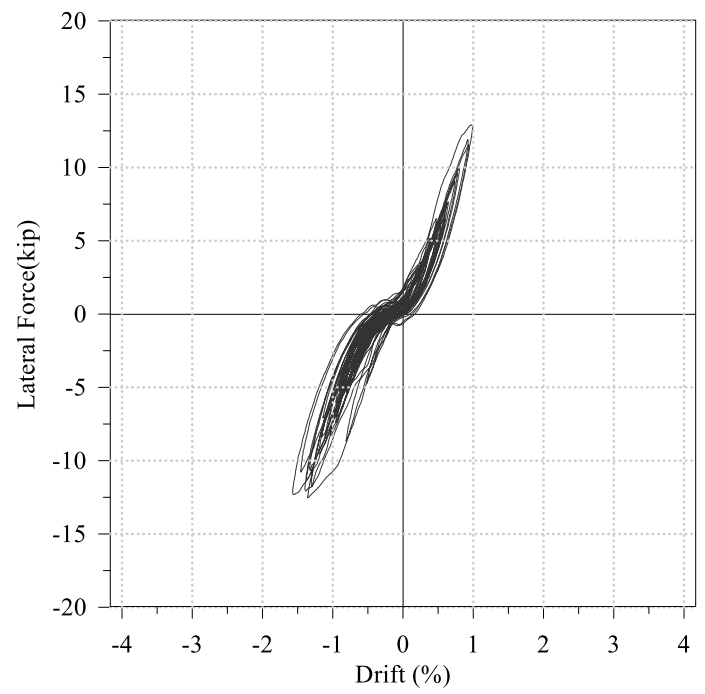

(c)

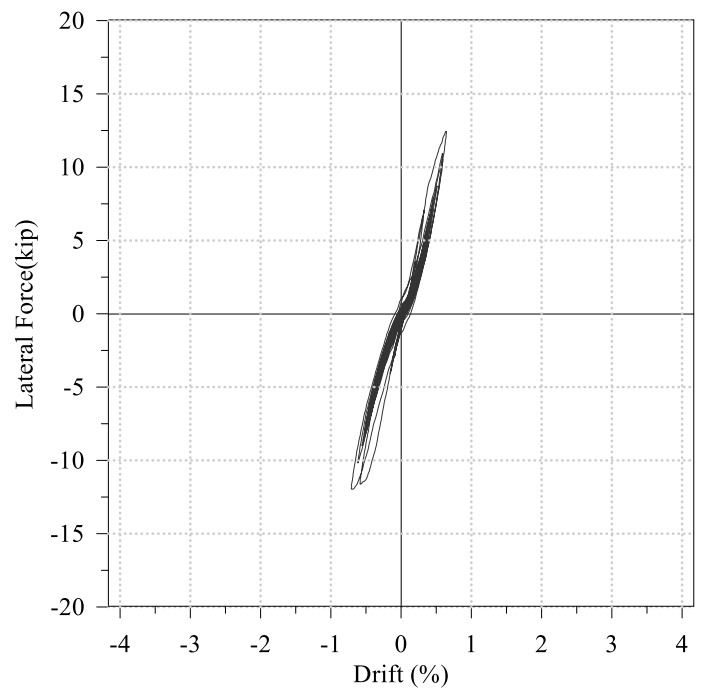

(b)

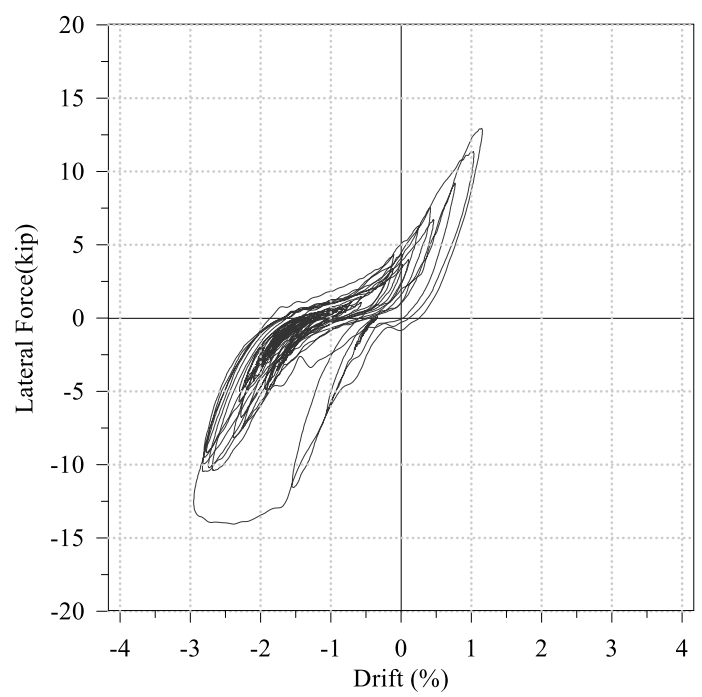

(d)

Figure 3.27 Earthquake response for W-FB: (a) SFGM-0.3, (b) SFGM-0.5, (c) SFGM-0.75, (d) SFGM-1.0. 

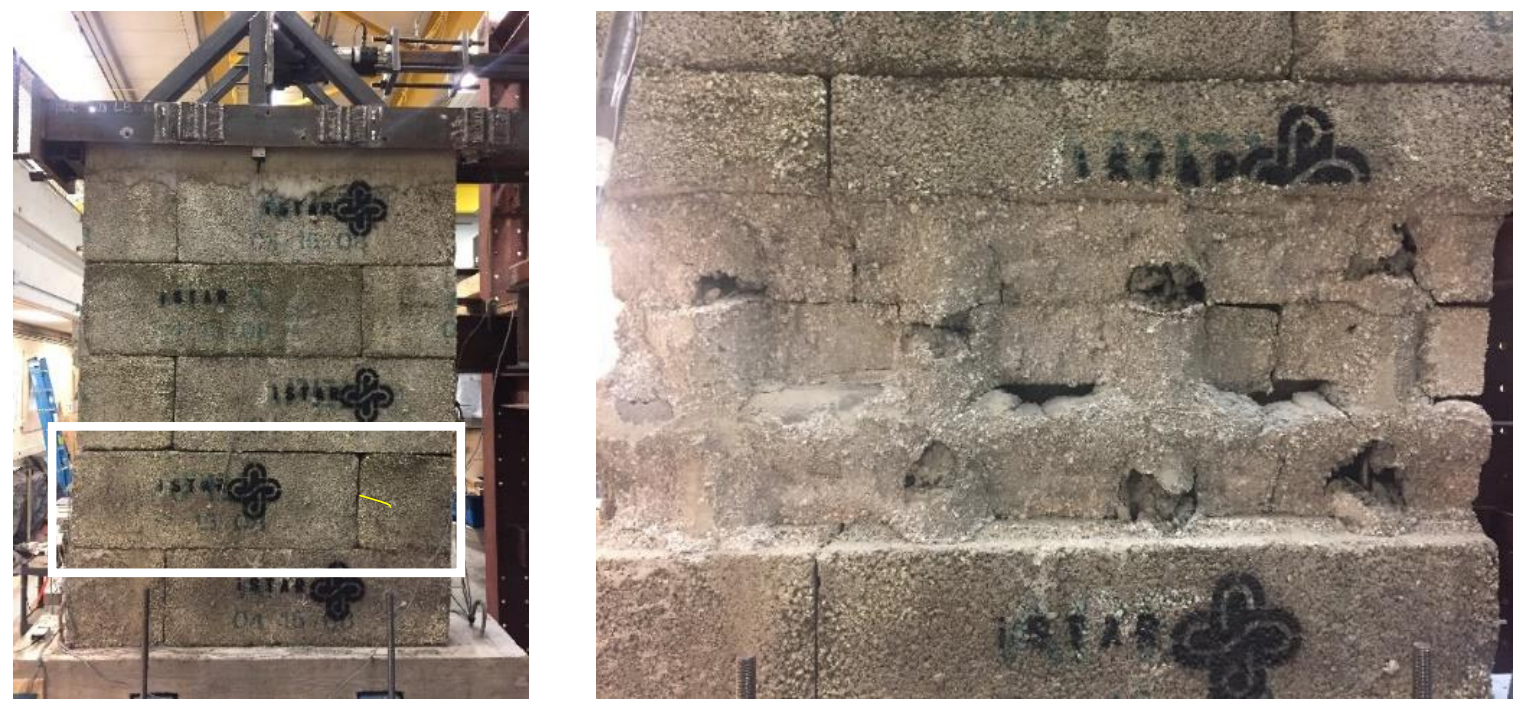

Figure 3.28 W-FB specimen with wall forms removed for inspection.

\subsubsection{Results Summary of SGICF Walls}

The backbone curves of W-RC and W-FB walls are shown in Figure 3.29. The experimental yield values were obtained from the reading of the strain gauges placed on the wall's vertical reinforcement of each side. Since the reinforcement of the walls was from the same steel batch, the reinforcement yield strain $\left(\varepsilon_{\text {sy }}\right)$ value was $2224 \mu \varepsilon$. The drift ratio of the $\mathrm{W}-\mathrm{RC}$ wall was $0.38 \%$ in the south direction at the first yield point, which was very close to the drift ratio $(0.39 \%)$ of $\mathrm{W}-\mathrm{FB}$ at the same direction. The drift ratio in the south direction exceeded $2.5 \%$ for both walls, which is a common drift limit in most structural design codes, but it was below $1.5 \%$ in the north direction as shown in Figure 3.30. The values of the ultimate points were assigned to the post maximum lateral strength of the walls when the reduction in strength reached $20 \%$, and the correspondent displacement was used to calculate walls displacement ductility. 

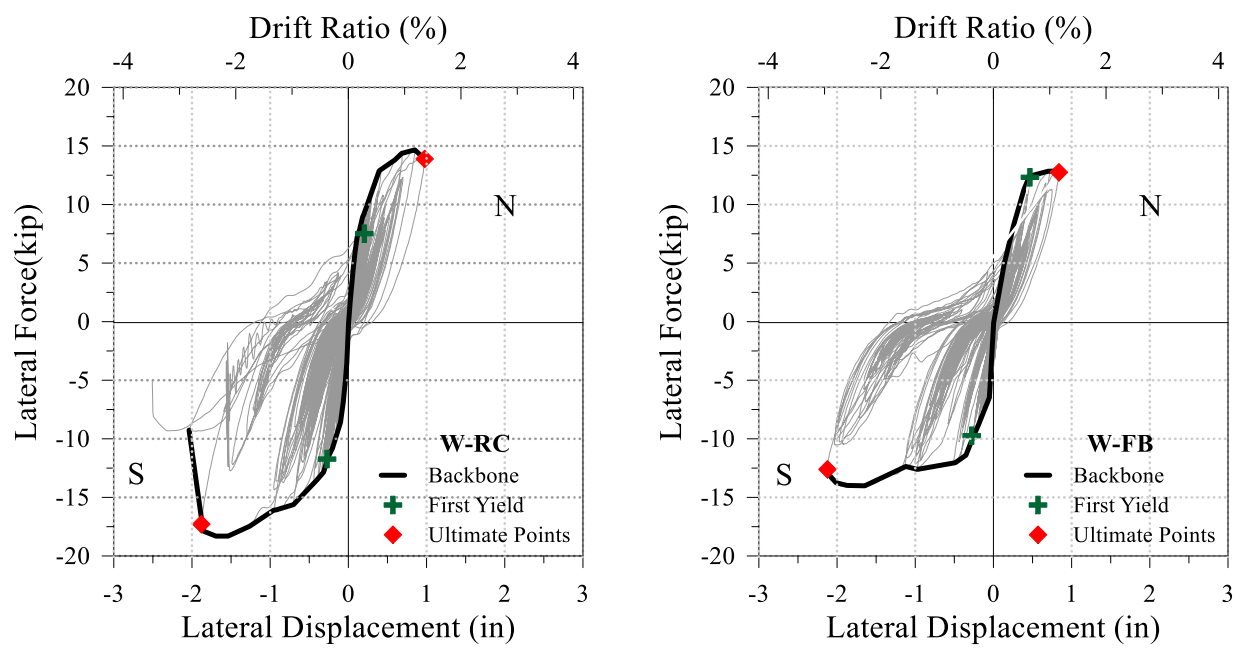

Figure 3.29 Experimental envelop curves for the SGICF walls.

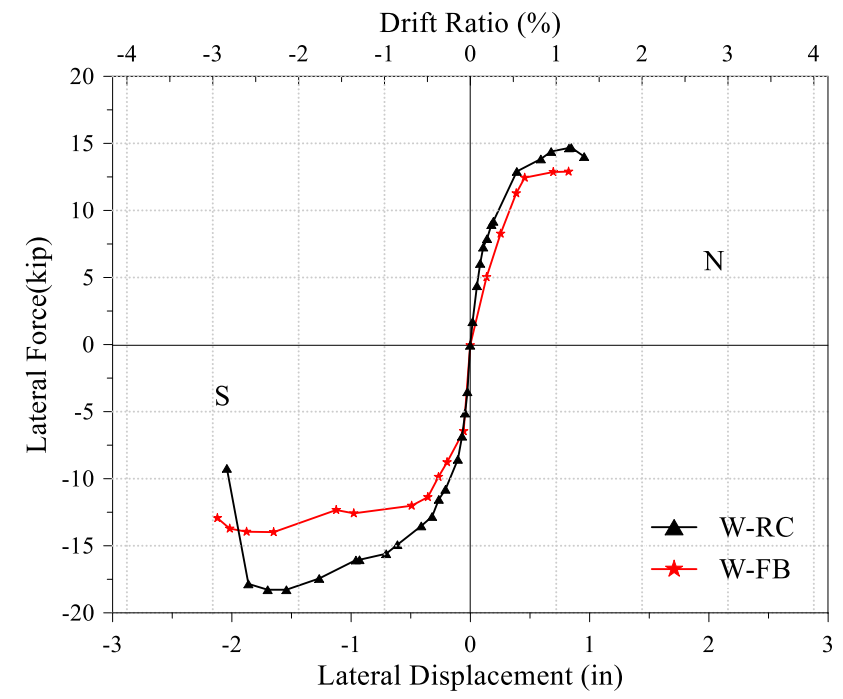

Figure 3.30 Backbone curves comparison of the SGICF walls.

The ductility of the SGICF walls was calculated using the following equation.

$$
\mu=\frac{\Delta_{u}}{\Delta y}
$$


Where, $\Delta_{\mathrm{u}}$ is the post peak displacement when the lateral strength of the wall has experienced a $20 \%$ reduction, and $\Delta_{\mathrm{y}}$ is the displacement when the first yield occur. The measured ductility of $\mathrm{W}-\mathrm{RC}$ was $90 \%$ of $\mathrm{W}-\mathrm{FB}$, which was calculated from the results obtained from the south direction. Table 3-3 shows the measured values for peak top wall lateral strength, displacement, drift, and ductility.

Table 3-3 Measured peak performance values for SGICF walls.

\begin{tabular}{|c|c|c|c|c|}
\hline Specimen & $\begin{array}{c}\text { Max. Lateral } \\
\text { Force (North) } \\
\text { (kip) }\end{array}$ & $\begin{array}{c}\text { Max. Lateral } \\
\text { Force (South) } \\
\text { (kip) }\end{array}$ & $\begin{array}{c}\text { Max. Drift } \\
\text { Ratio (\%) }\end{array}$ & $\begin{array}{c}\text { Max. Displacement } \\
\text { Ductility }(\mu)\end{array}$ \\
\hline W-RC & 14.7 & -18.2 & 2.8 & 6.8 \\
\hline W-FB & 12.9 & -14 & 2.9 & 7.5 \\
\hline
\end{tabular}

The wall with steel fiber-reinforced concrete resulted in lower strength than that with reinforced concrete despite a similarity in the basic material properties and layout of the walls as shown in Figure 3.30. This reduction can be attributed to the voids in the cores created by the poor consolidation of the steel fiber-reinforced concrete, which introduced weakness in the wall. The two walls were constructed at the same time, using similar techniques and using concrete of similar workability as measured by the slump test, and yet the concrete with fibers did not appear to sufficiently flow through the SGICF cores. 
The stiffness of each wall was measured before and after the application of each scaled ground motion according to the following equation.

$$
\mathrm{K}=\frac{4 \cdot \pi^{2}}{\mathrm{~T}_{\mathrm{n}}^{2}} \cdot \mathrm{m}
$$

Where, $\mathrm{m}$ is the inertia mass; and $\mathrm{T}_{\mathrm{n}}$ is the natural frequency period of the wall that was obtained from the free vibration tests. A summary of wall's stiffness degradation is shown in Table 3-4. Figure 3.31 shows the comparison of wall's stiffness degradation and the increase in the natural period. It was clear that the degradation of W-FB stiffness was rapid due to the voids found in the specimen due to the lack of the concrete consolidation.

Table 3-4 Measured stiffness of SGICF walls from the free vibration tests.

\begin{tabular}{|c|c|c|c|c|c|}
\hline Event & SF-GM & $\begin{array}{c}\text { Period } \mathrm{T}_{\mathrm{n}}(\mathrm{sec}) \\
\text { W-RC }\end{array}$ & $\begin{array}{c}\text { Stiffness } \\
\text { ( kip/in) }\end{array}$ & $\begin{array}{c}\text { Period } \mathrm{T}_{\mathrm{n}}(\mathrm{sec}) \\
\text { W-FB }\end{array}$ & $\begin{array}{c}\text { Stiffness } \\
\text { ( kip/in) }\end{array}$ \\
\hline FV1 & 0.3 & 0.28 & 54.4 & 0.3 & 47.4 \\
\hline FV2 & 0.5 & 0.32 & 41.6 & 0.39 & 28 \\
\hline FV3 & 0.75 & 0.38 & 29.5 & 0.47 & 19.3 \\
\hline FV4 & 1.0 & 0.48 & 18.5 & 0.55 & 14.1 \\
\hline FV5 & 1.25 & 0.51 & 16.4 & - & - \\
\hline
\end{tabular}



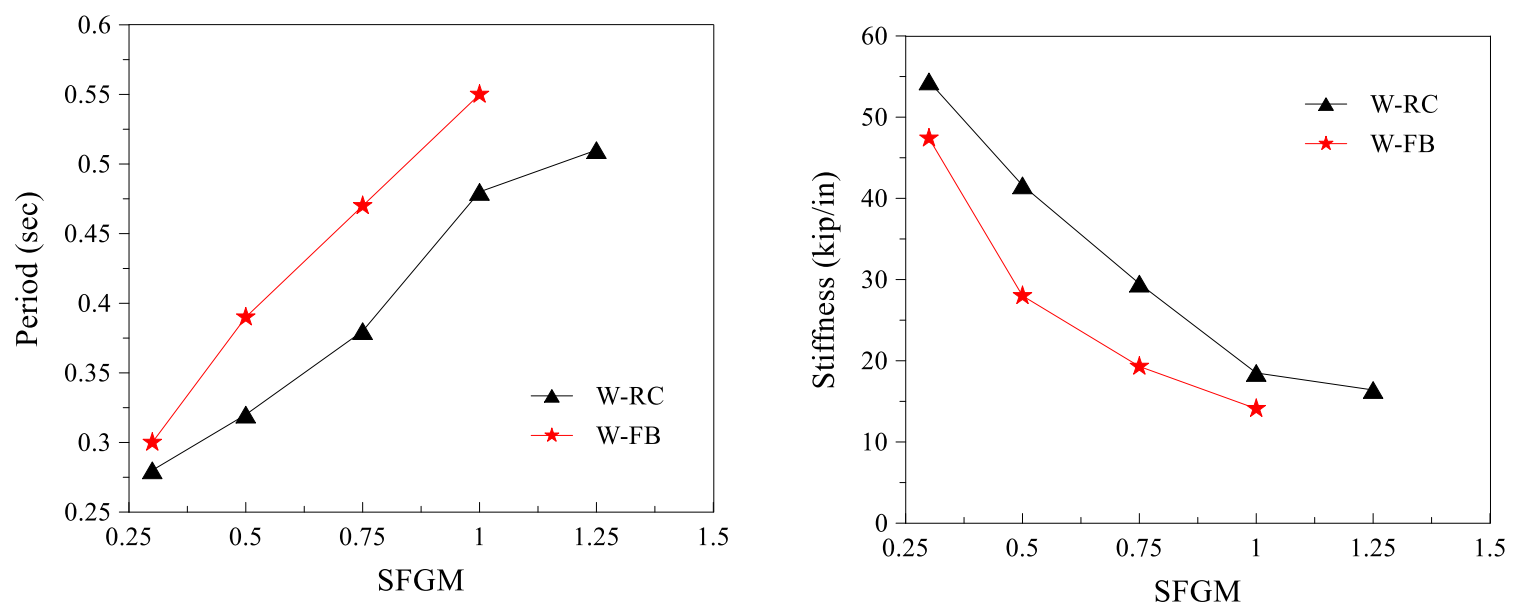

Figure 3.31 Comparison of the natural period and stiffness of SGICF walls.

The wall with steel fiber-reinforced cores also resulted in higher drift under the same ground motion intensity as shown in Figure 3.32. The higher drifts are a result of lower stiffness and lower strength resulting from the voids in the cores made from the fiber reinforced concrete.

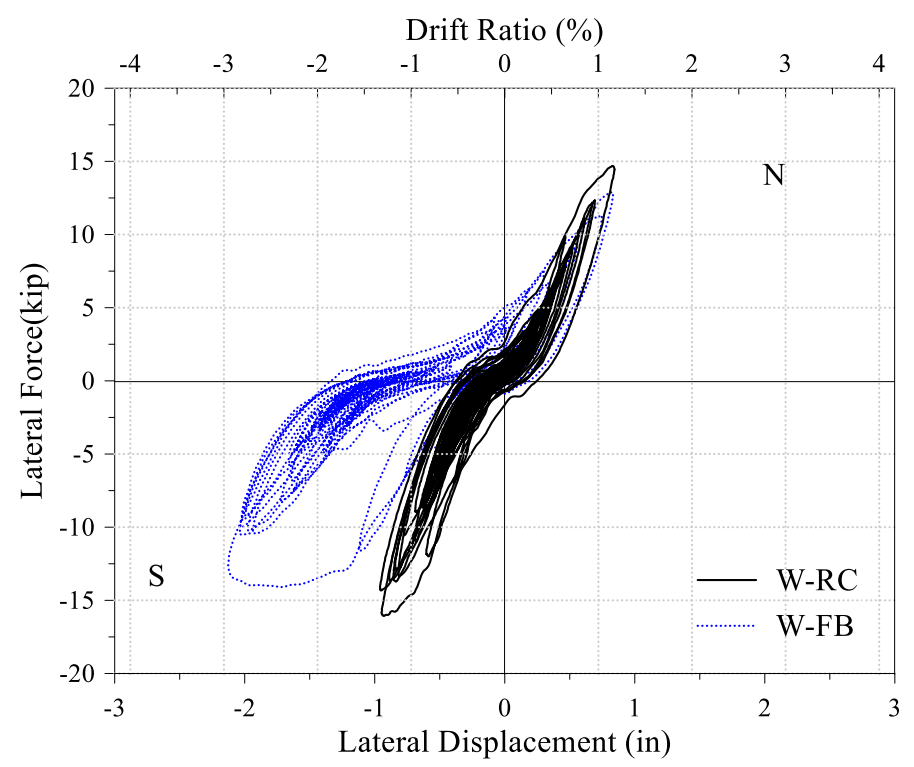

Figure 3.32 Hysteresis comparison to 1.0 scaled ground motion of SGICF walls. 


\subsubsection{The New Pattern of SGICF Walls}

The failure modes for G-RC and G-FB walls were the same during the low scale factors of the ground motion. Observation of cracks and damage was clear since no wall forms existed. Flexural cracks around the connections between the vertical and horizontal cores dominated the failure modes within the low scale motions. During the high scale factors of the ground motion, the width of the flexural cracks increased, and the distribution of the shear cracks was pronounced in both the horizontal and vertical cores.

\subsubsection{G-RC}

Figure 3.33 shows the G-RC specimen after undergoing four different scaled ground motions. The initial natural period of vibration was 0.35 second, and had a damping ratio of $1.46 \%$ as recorded from the free vibration. After the application of the ground motion scaled to 0.3 , flexural cracks started to generate at the joint faces of the vertical and horizontal cores where the flexural hinges expected to generate due to the high moment values. At this level of motion, specimen drift percent was $0.57 \%$ as shown in Figure 3.34a. At the end of the ground motion scaled to 0.75, shear cracks were observed at some of the horizontal cores. At this level of motion, the recorded lateral strength was 14 kips, and the drift reached $2.42 \%$ as shown in Figure 3.34c.

It was noticed that when the specimen was subjected to 1.0 scaled ground motion, the degradation of the lateral strength was at an average of 0.89 Vmax. Since the degradation in the lateral strength did not go below $0.8 \mathrm{Vmax}$, the following ground motion was applied and scaled to 1.25 of the earthquake record, and resulted in strength 
degradation of $0.67 \mathrm{Vmax}$. Although the measured lateral strength of the wall in the north direction was 13 kips, a decision was made to terminate the test because the lateral strength of the specimen in the south direction (inertia mass side) was 9.4 kips.

At the end of the ground motion scaled to 1.25 , the outer vertical cores near the load beam underwent severe shear cracks, and concrete spalled as shown in Figure 3.35. Flexural crack width near the joints increased with some concrete spalling around the fourth level of the horizontal cores. Also, the joints at the first level of the horizontal cores did not show high damage when compared to the subsequent levels.

The natural period of vibration of the specimen degraded from 0.35 second to 0.98 second after completion of the ground motion scaled to 1.25 , and the damping ratio increased from $1.46 \%$ to $4.2 \%$. The maximum lateral strength was 14 kips and drift percent was 3.88\% at the end of the test as shown in Figure 3.36. After securing the specimen and the inertia masses, a thorough investigation was made to conclude the damage mode at the end of the last applied scaled ground motion. One of the critical observations was that increasing the length of the horizontal cores delayed the onset of the shear cracks at these cores, and improved the lateral drift capacity of the wall. 


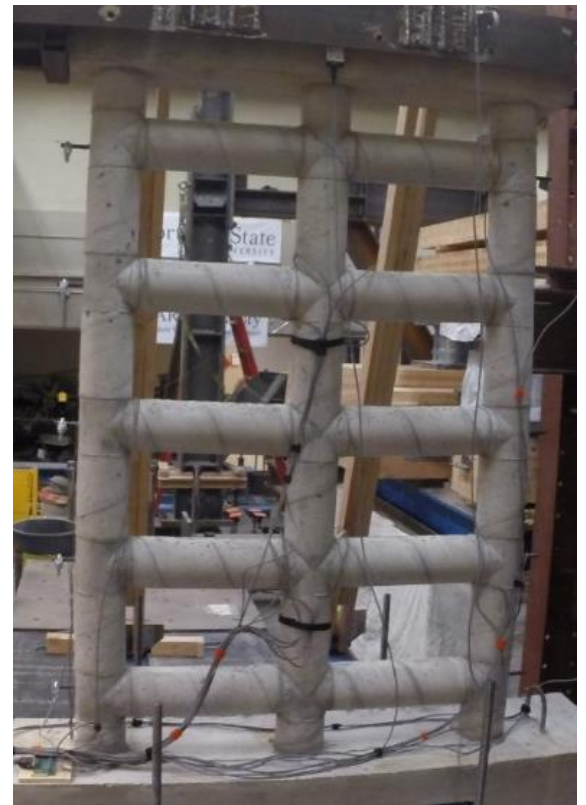

(a)

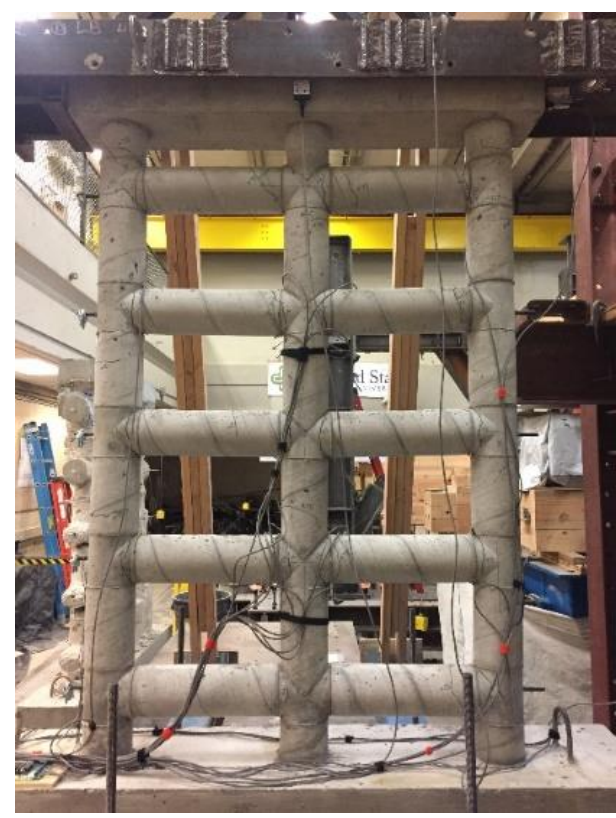

(c)

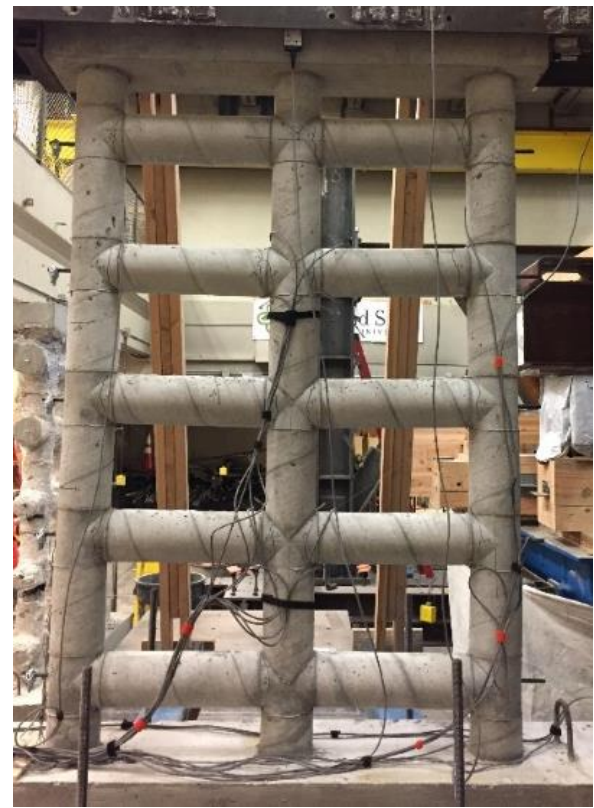

(b)

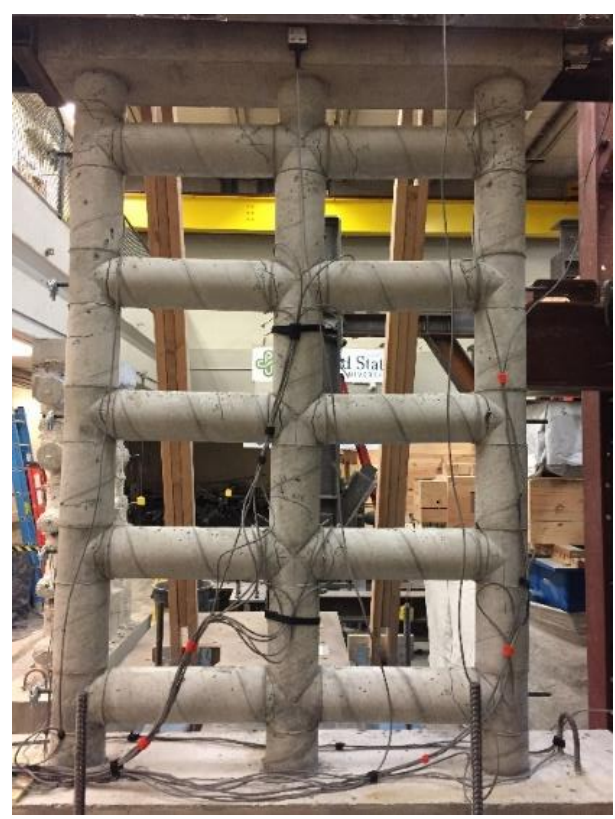

(d)

Figure 3.33 Failure mode of G-RC: (a) SFGM-0.3, (b) SFGM-0.5, (c) SFGM-0.75, (d) SFGM-1.0. 


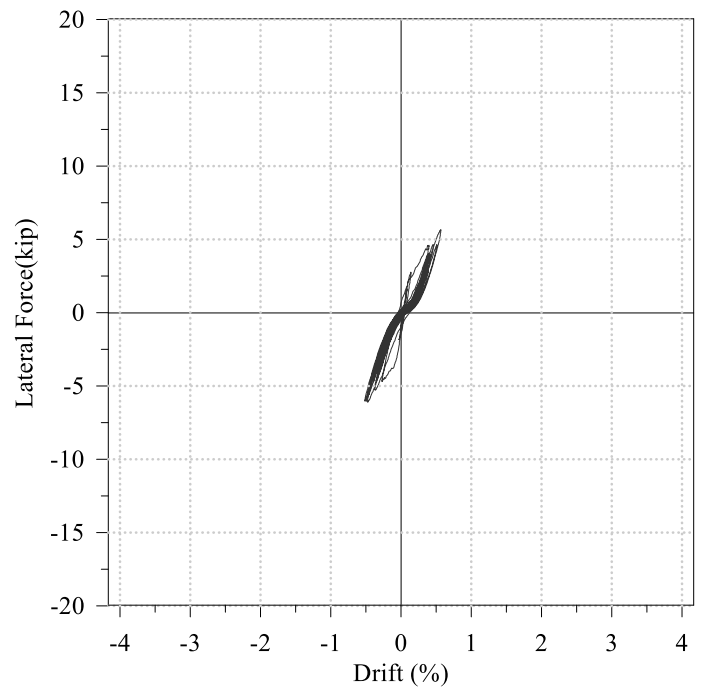

(a)

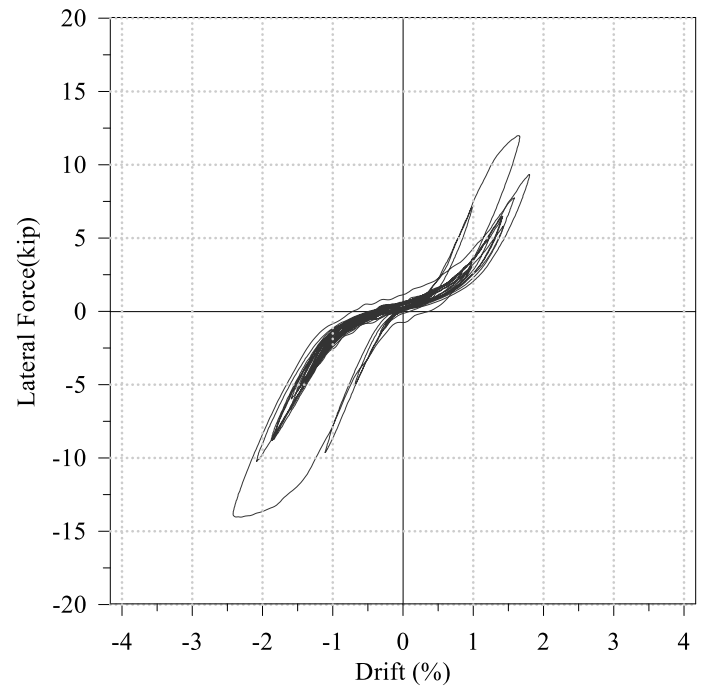

(c)

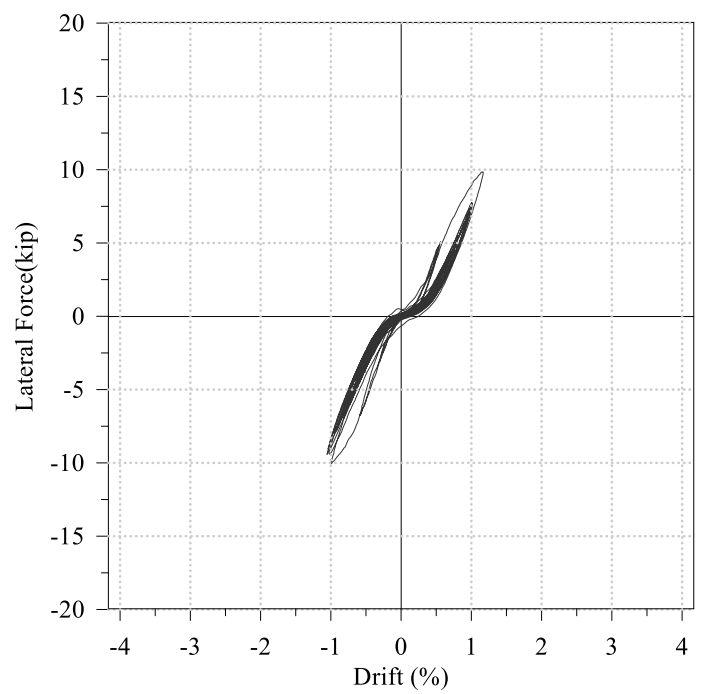

(b)

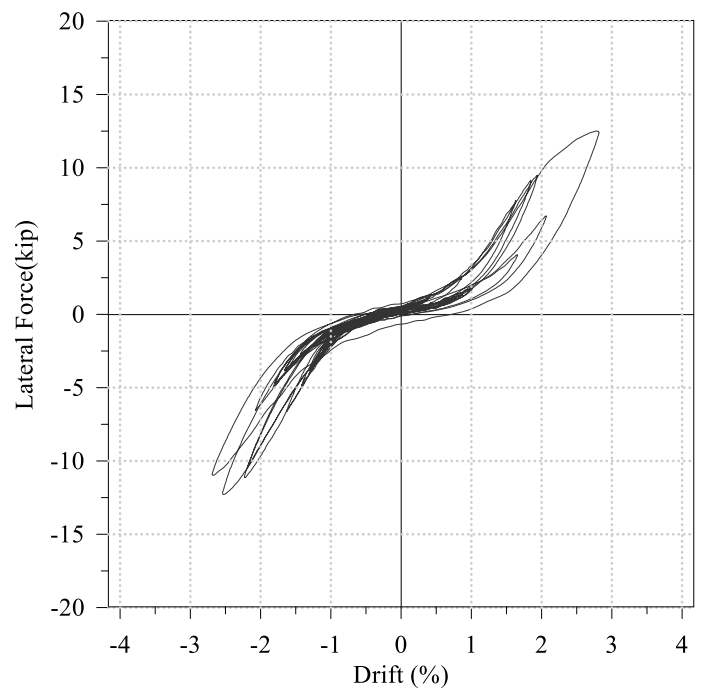

(d)

Figure 3.34 Earthquake response for G-RC: (a) SFGM-0.3, (b) SFGM-0.5, (c) SFGM-0.75, (d) SFGM-1.0. 


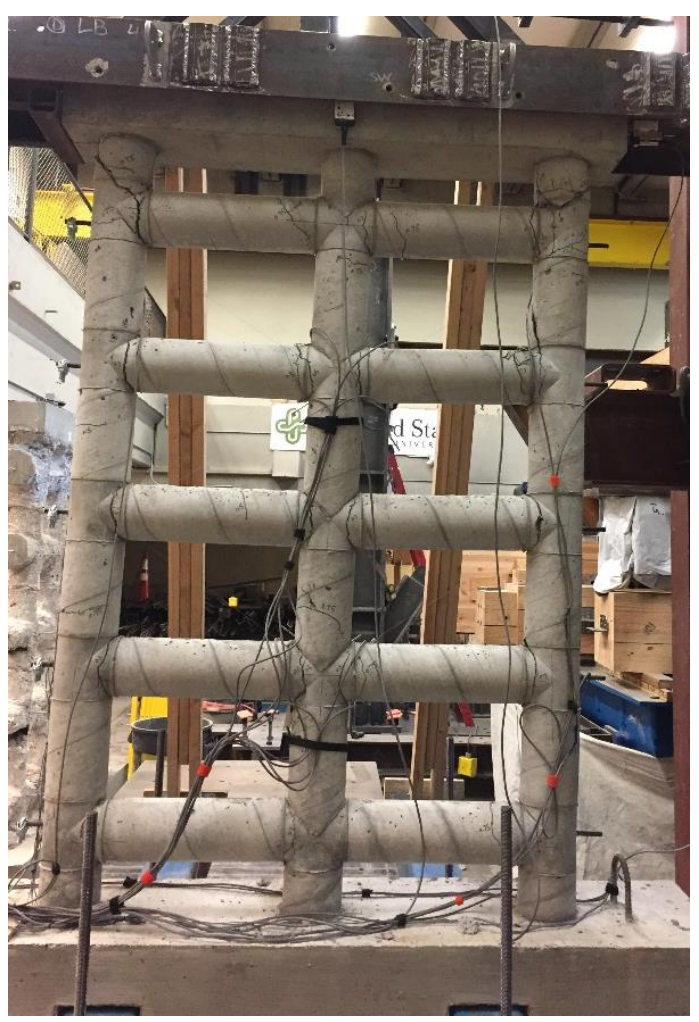

Figure 3.35 Failure mode of G-RC wall at SFGM-1.25.

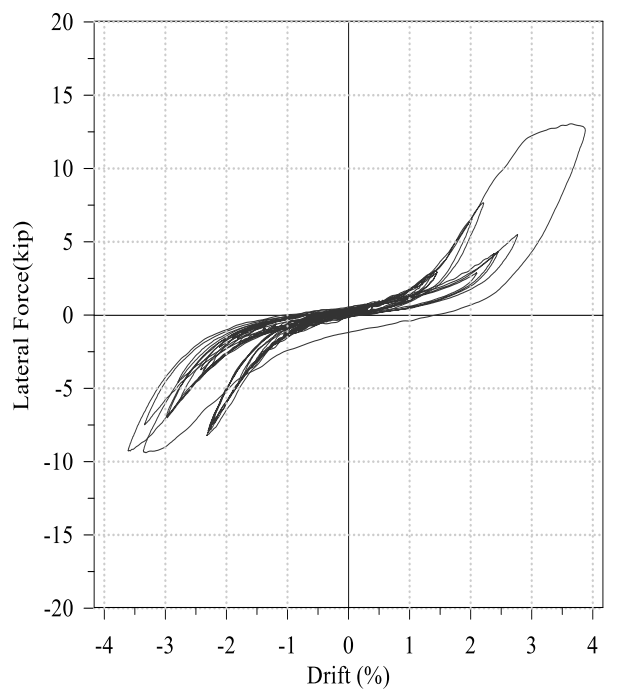

Figure 3.36 Hysteresis response of G-RC wall to 1.25 scaled ground motion. 


\subsubsection{G-FB}

After removing all of the form works from G-FB wall, a concrete honeycomb was observed in some horizontal cores as shown in Figure 3.37. This was kept without treatment to follow the general practice of expecting concrete consolidation problems in ICF walls. Unlike the other tested walls, the G-FB was subjected to seven scaled ground motions. Figure 3.38 shows the specimen after undergoing six different scaled ground motions that ranged from 0.3-1.5.

The initial natural period of vibration was 0.38 second and a damping ratio of $1.51 \%$ as recorded from the free vibration. After the application of the ground motion was scaled to 0.3 , flexural cracks started to generate, similarly to G-RC wall, at the joint faces of the vertical and horizontal cores where high flexural moments were expected. At this level of motion, the specimen drift percent was $0.76 \%$ as shown in Figure 3.40a. When the wall subjected to 0.5 scaled ground motion, and where the concrete honeycomb occurred, visibile shear cracks were generated at the third level of the horizontal cores.

During the subsequent scaled ground motion events, G-FB experienced an increase in flexural cracks width and spreading of the shear cracks in both the vertical and horizontal cores. At the scaled ground motion of 1.5, the concrete separated from the horizontal core where the shear cracks started but did not spall due to the influence of the steel fiber as shown in Figure 3.38f. At this level of motion, the recorded drift ratio was $4.85 \%$, and the maximum lateral strength was 12.5 kips. Lateral strength degradation was noticed at this level of ground motion with an average of $0.95 \mathrm{Vmax}$. 
Subsequent ground motion scaled to 1.75 of the earthquake record resulted in the complete collapse of the specimen. As shown in Figure 3.39, the concrete crushed around the top joints, and the top level of the specimen collapsed by separating off the rest of the wall.

The natural period of vibration of the specimen degraded from 0.38 second to 0.75 second after the completion of the ground motion was scaled to 1.5 , and the damping ratio was increased from $1.51 \%$ to $4.32 \%$. The maximum lateral strength was 12.5 kips and drift percent was $8.3 \%$ at the end of the test as shown in Figure 3.41.

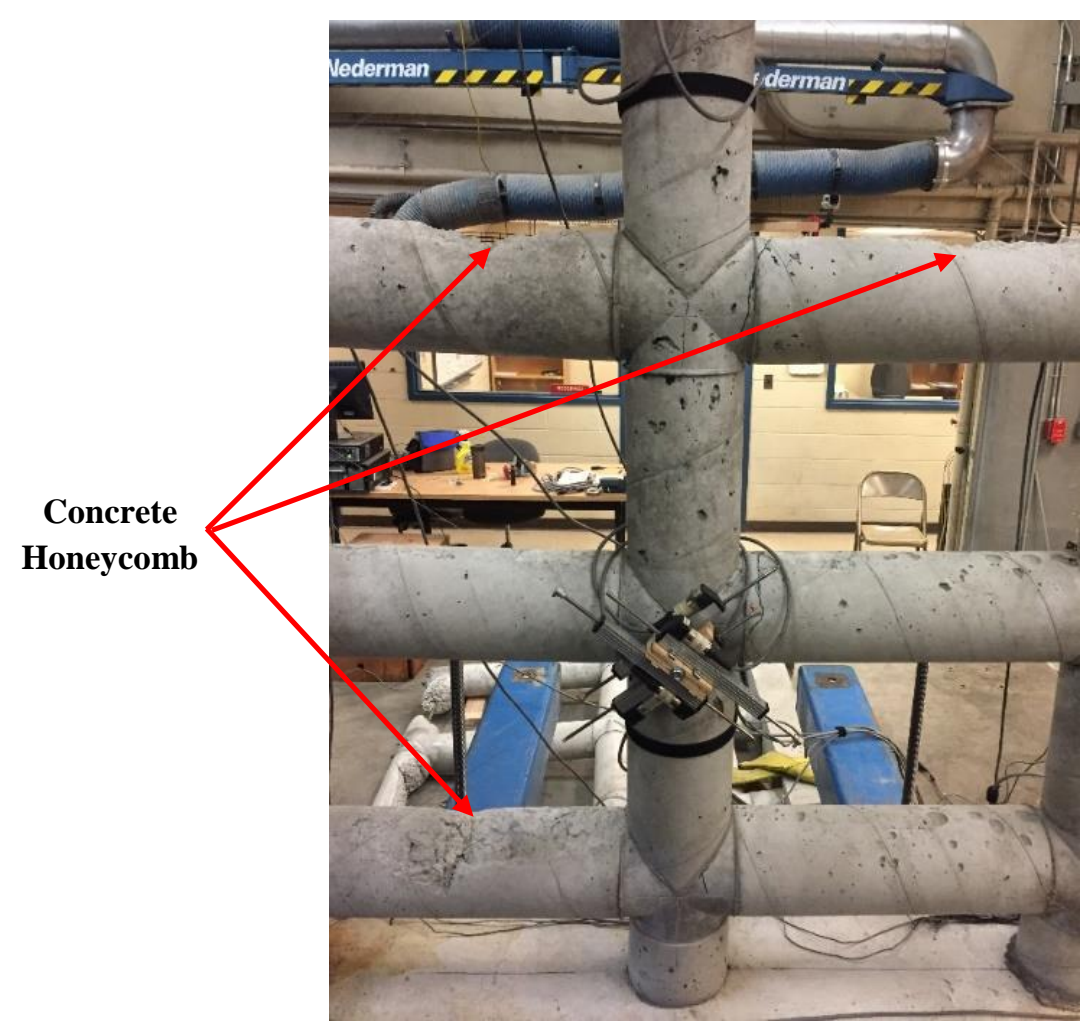

Figure 3.37 Lack of concrete consolidation in G-FB specimen. 


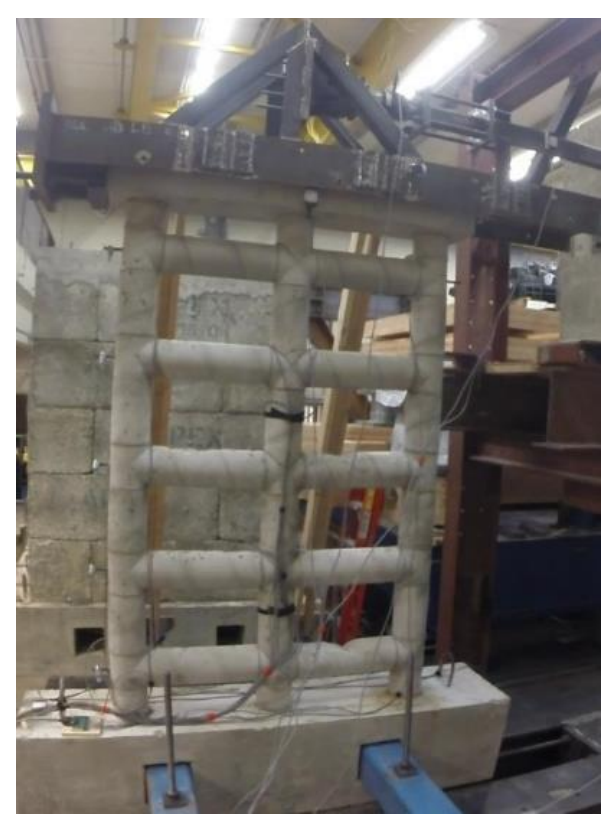

(a)

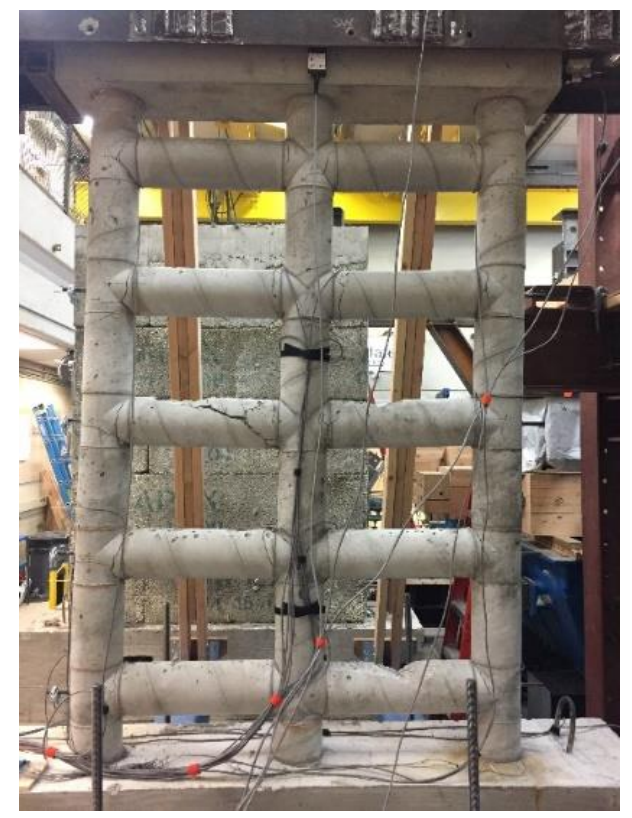

(c)

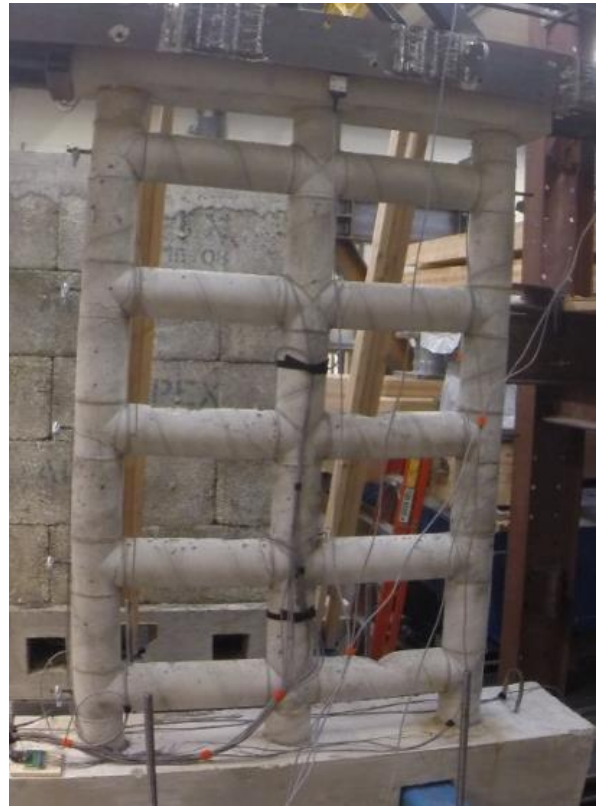

(b)

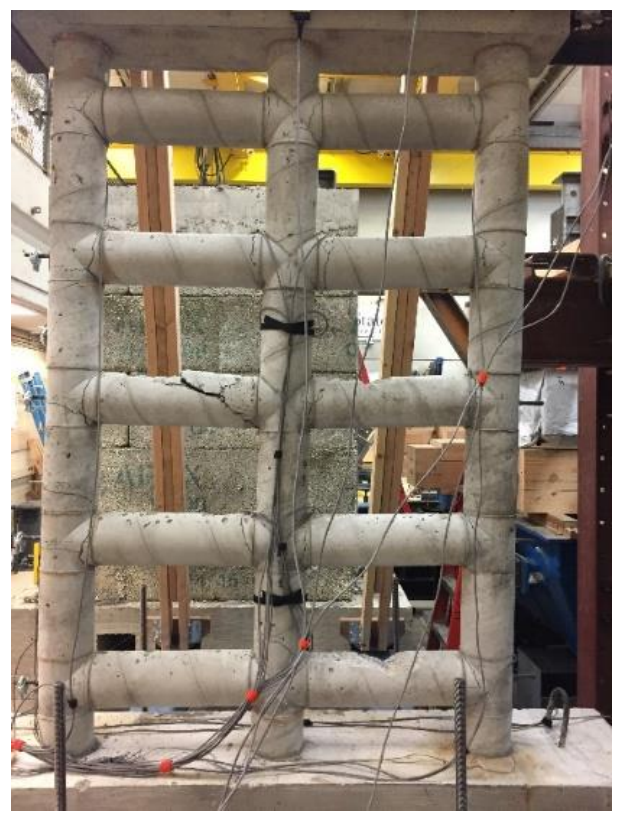

(d) 


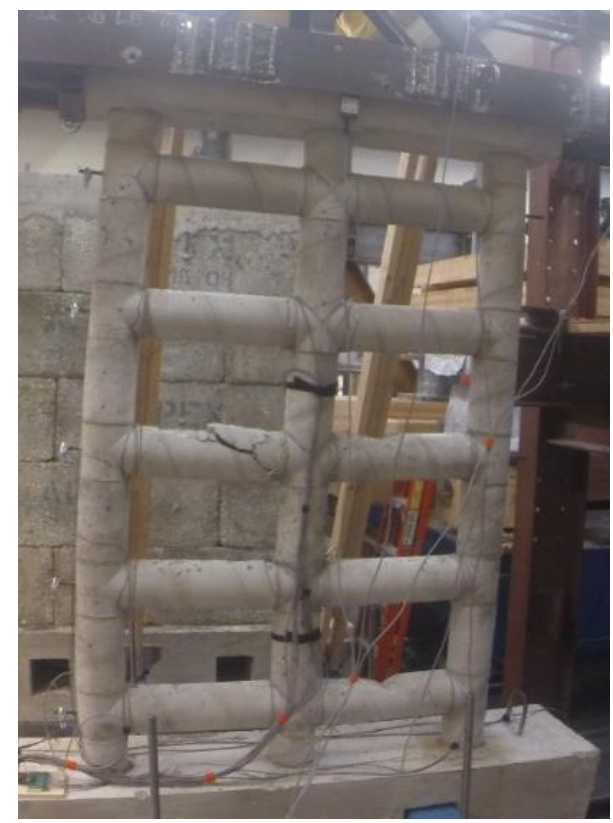

(e)

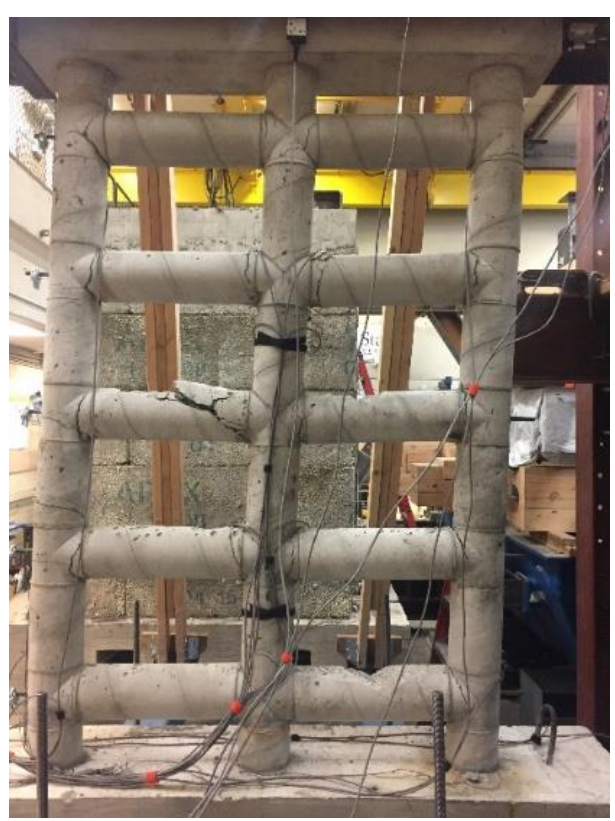

(f)

Figure 3.38 Failure mode of G-FB: (a) SFGM-0.3, (b) SFGM-0.5, (c) SFGM-0.75, (d) SFGM-1.0, (e) SFGM-1.25, (f) SFGM-1.5.

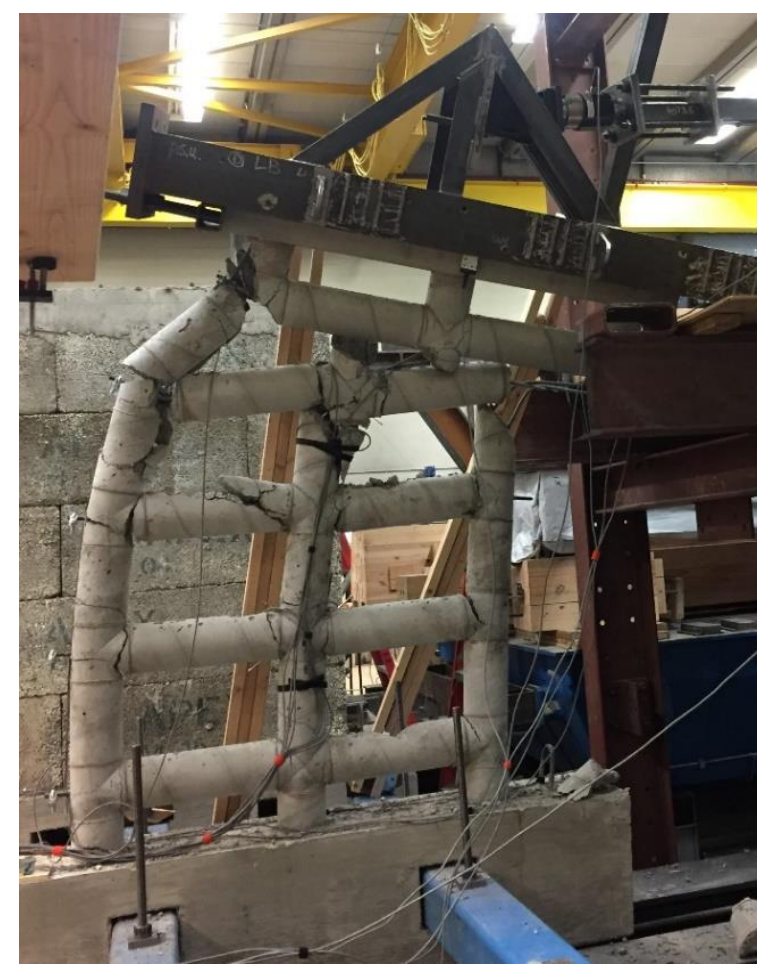

Figure 3.39 Collapse of G-FB wall at SFGM-1.75. 


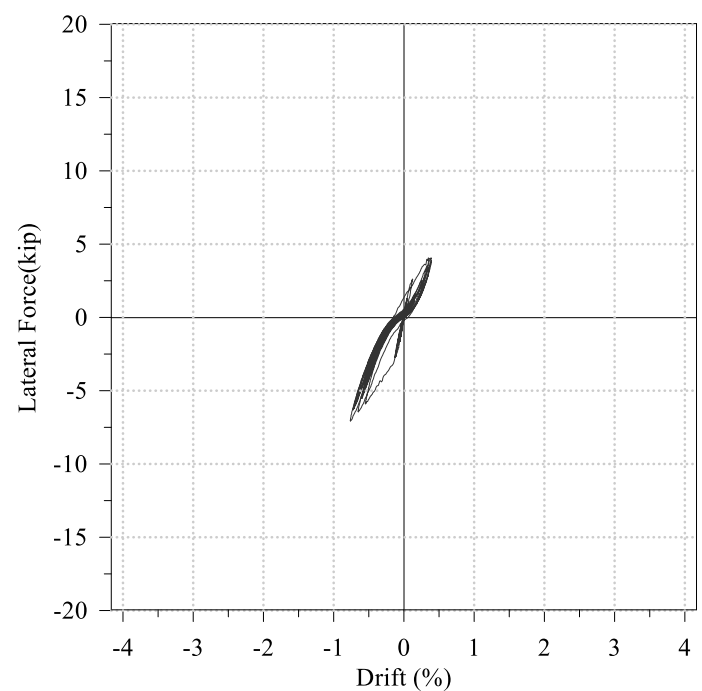

(a)

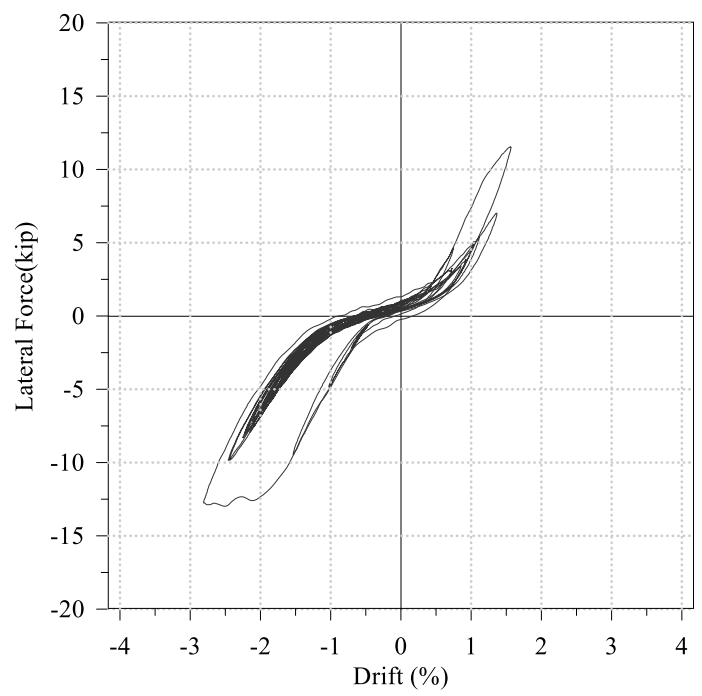

(c)

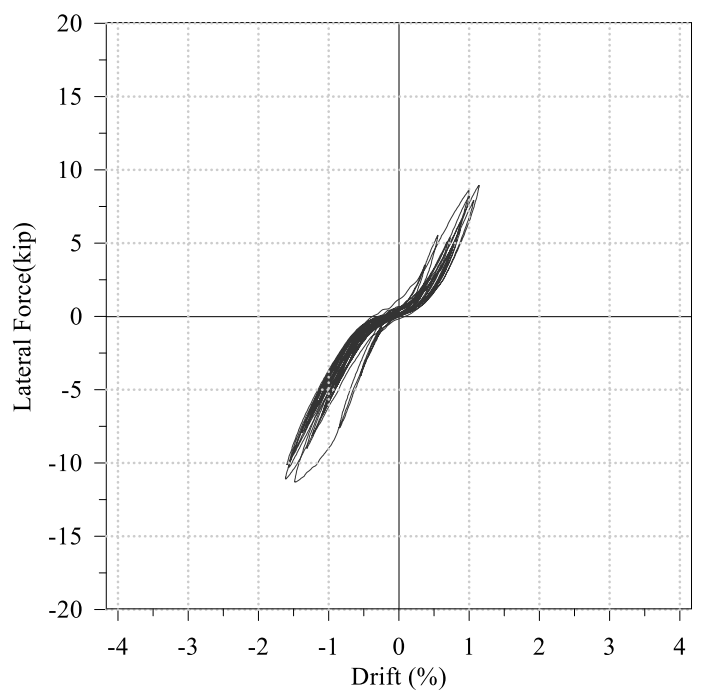

(b)

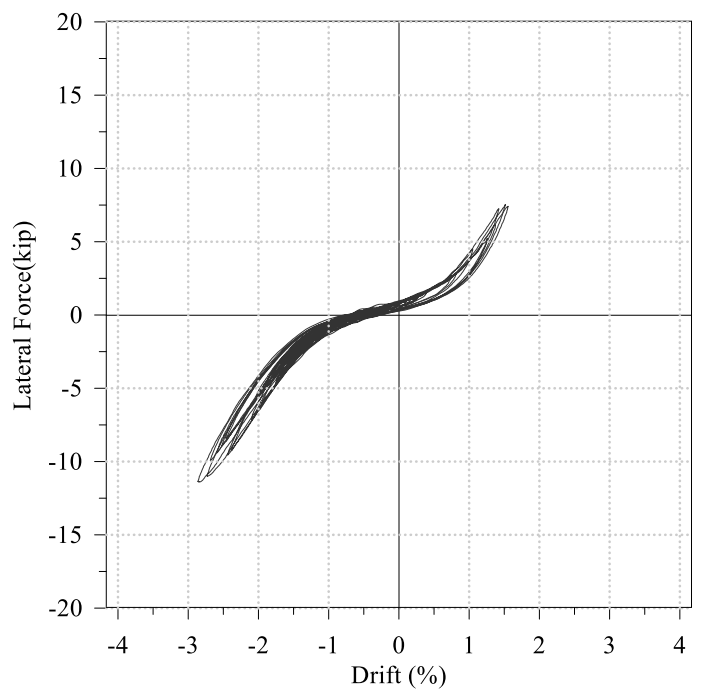

(d) 


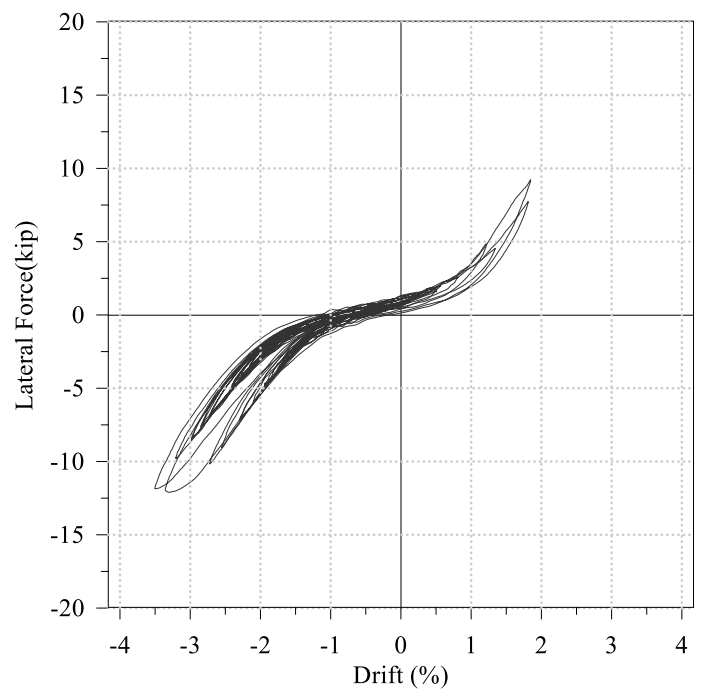

(e)

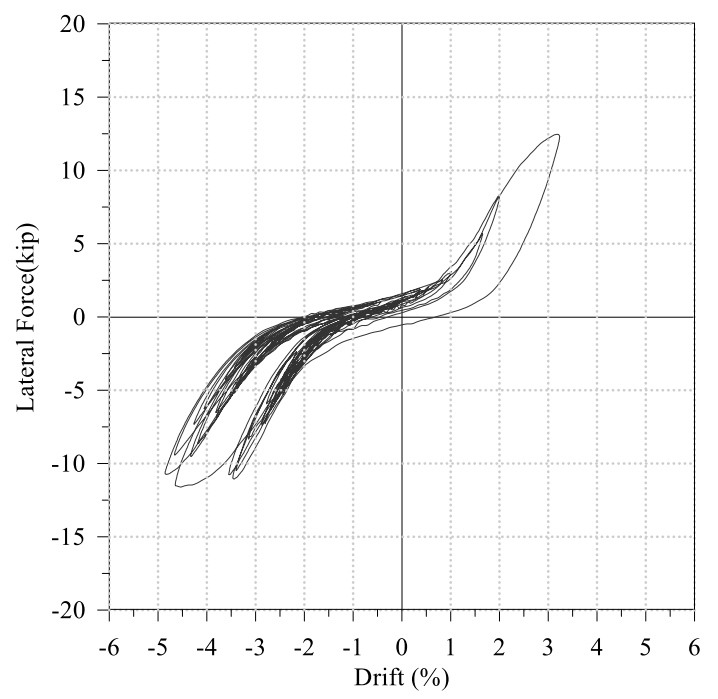

(f)

Figure 3.40 Earthquake response for G-FB: (a) SFGM-0.3, (b) SFGM-0.5, (c) SFGM-0.75, (d) SFGM-1.0, (e) SFGM-1.25, (f) SFGM-1.5.

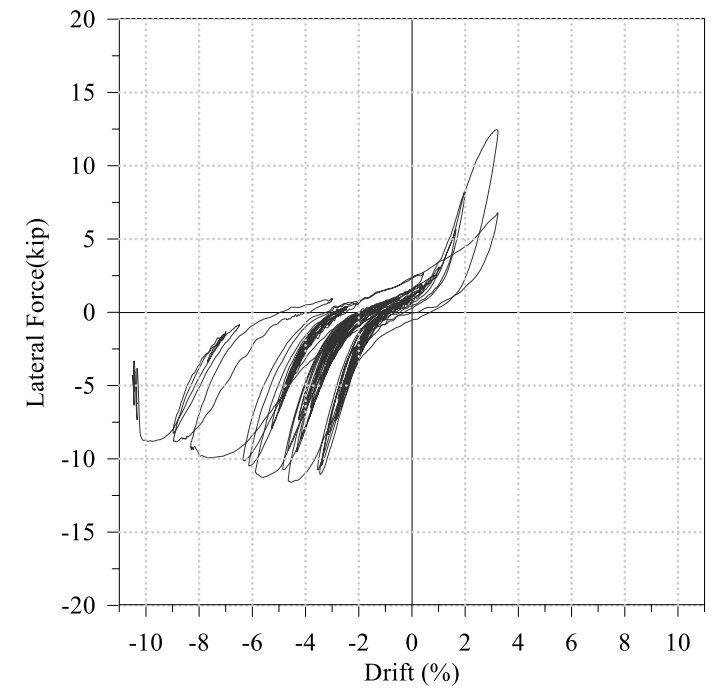

Figure 3.41 Hysteresis response of G-FB wall to 1.75 scaled ground motion. 


\subsubsection{Results Summary of The New SGICF Walls}

The backbone curves of G-RC and G-FB walls are shown in Figure 3.42. The experimental yield values were obtained from the reading of the strain gauges placed on the wall's vertical reinforcement of each side. Yield points were assigned when the strain of the reinforcement reached $2224 \mu$ s. The drift ratio of the G-RC wall was $0.94 \%$ in the south direction at the first yield point, and $1.16 \%$ for W-FB at the same direction. The drift ratio in the south direction exceeded 3.5\% for G-RC wall and $6 \%$ for G-FB. The values of the ultimate points were assigned to the post maximum lateral strength of the walls when the reduction in strength reached $20 \%$, and the correspondent displacement was used to calculate walls displacement ductility. Despite the fact that both G-RC and G-FB walls showed higher drift ratio than the W-RC and W-FB walls, displacement ductility was smaller. The displacement ductility was 2.64 and 5.5 for G-RC and G-FB, respectively.
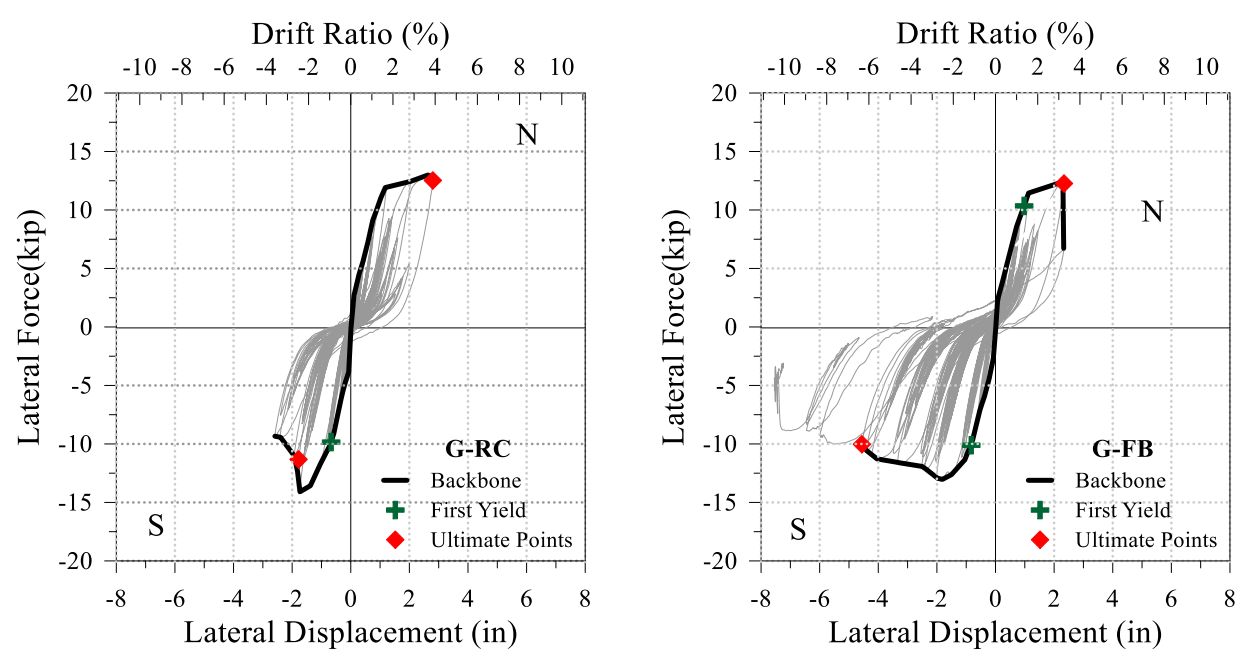

Figure 3.42 Experimental envelop curves for G-RC and G-FB walls. 
Figure 3.42 shows the hysteresis envelop for both G-RC and G-FB walls. The variation in the lateral strength of the walls in both directions was small. Table 3-5 shows the measured values for peak top wall lateral strength, displacement, drift ratio, and ductility.

The stiffness of each wall was measured before and after the application of each scaled ground motion by using Eq. 3.3. A comparison of wall's stiffness degradation and the increase in the natural period are shown in Figure 3.43. It is shown that G-RC stiffness degraded faster than G-FB wall. A summary of wall's stiffness degradation is shown in Table 3-6. The results showed in Table 3-6 support the conclusion that the lateral displacement that improved in the G-FB wall was due the use of the steel fiber when compared to the wall with reinforced concrete only.
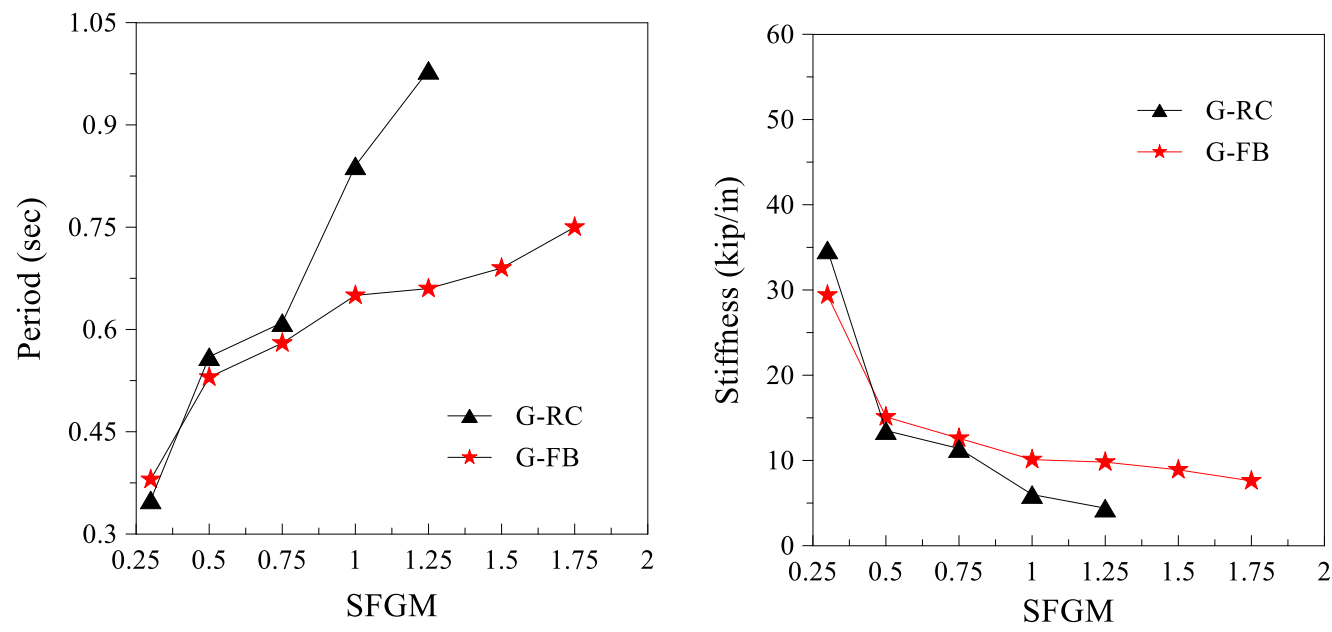

Figure 3.43 Comparison of the natural period and stiffness of the new pattern of SGICF walls. 
The obtained results showed that the steel fiber in the G-FB wall did not improve the lateral strength of the wall. Further, both walls showed a close strength and drift ratio in the south direction at a scaled ground motion of 1.25, as shown in Figure 3.44. Even though the steel fiber in the G-FB wall did not improve the lateral strength of the wall, a notable improvement in the lateral displacement was recorded as shown in Figure 3.45

Table 3-5 Measured peak performance values for the new pattern SGICF walls.

\begin{tabular}{|c|c|c|c|c|}
\hline Specimen & $\begin{array}{c}\text { Max. Lateral } \\
\text { Force (North) } \\
\text { (kip) }\end{array}$ & $\begin{array}{c}\text { Max. Lateral } \\
\text { Force (South) } \\
\text { (kip) }\end{array}$ & $\begin{array}{c}\text { Max. Drift } \\
\text { Ratio (\%) }\end{array}$ & $\begin{array}{c}\text { Max. Displacement } \\
\text { Ductility }(\mu)\end{array}$ \\
\hline G-RC & 13 & -14 & 3.9 & 2.64 \\
\hline G-FB & 12.5 & -13 & 6.35 & 5.5 \\
\hline
\end{tabular}

Table 3-6 Measured stiffness of the new pattern SGICF walls from the free vibration tests.

\begin{tabular}{|c|c|c|c|c|c|}
\hline Event & SF-GM & $\begin{array}{c}\text { Period } \mathrm{T}_{\mathrm{n}}(\mathrm{sec}) \\
\text { G-RC }\end{array}$ & $\begin{array}{c}\text { Stiffness } \\
\text { ( kip/in) }\end{array}$ & $\begin{array}{c}\text { Period } \mathrm{T}_{\mathrm{n}}(\mathrm{sec}) \\
\text { G-FB }\end{array}$ & $\begin{array}{c}\text { Stiffness } \\
\text { ( kip/in) }\end{array}$ \\
\hline FV1 & 0.3 & 0.35 & 34.7 & 0.38 & 29.4 \\
\hline FV2 & 0.5 & 0.56 & 13.5 & 0.53 & 15.1 \\
\hline FV3 & 0.75 & 0.61 & 11.4 & 0.58 & 12.6 \\
\hline FV4 & 1.0 & 0.84 & 6.0 & 0.65 & 10.1 \\
\hline FV5 & 1.25 & 0.98 & 4.4 & 0.66 & 9.8 \\
\hline FV6 & 1.5 & - & - & 0.69 & 8.9 \\
\hline FV7 & 1.75 & - & - & 0.75 & 7.6 \\
\hline
\end{tabular}




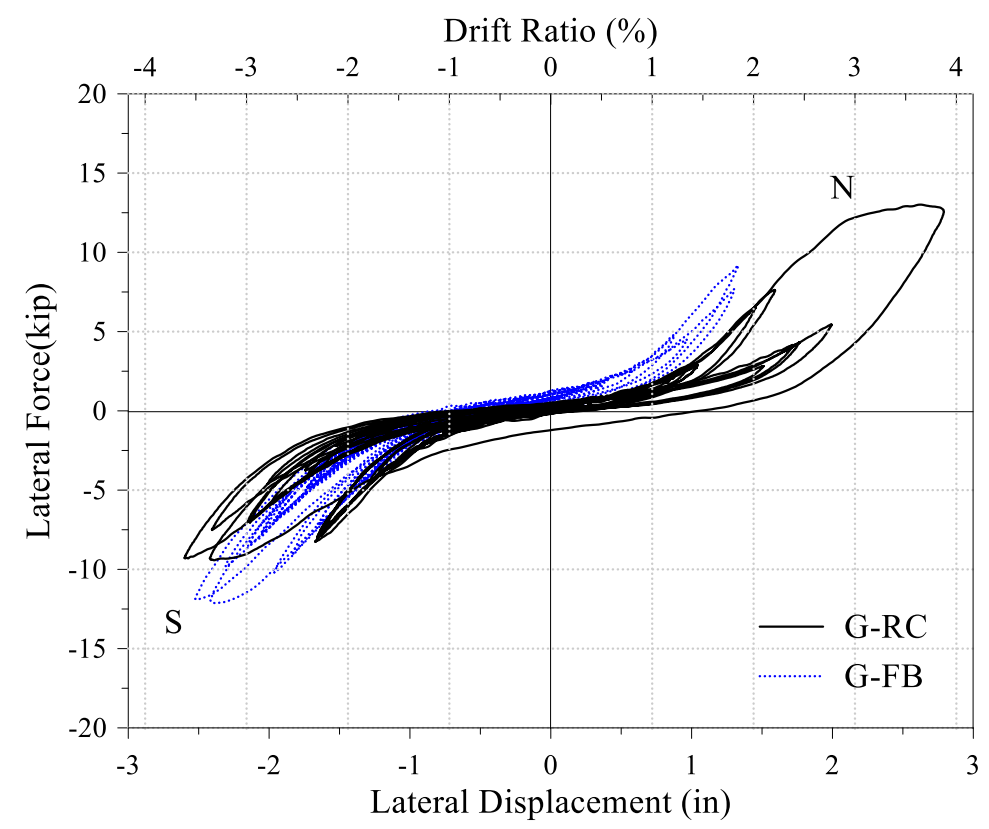

Figure 3.44 Hysteresis comparison to 1.25 scaled ground motion for the new pattern SGICF walls.

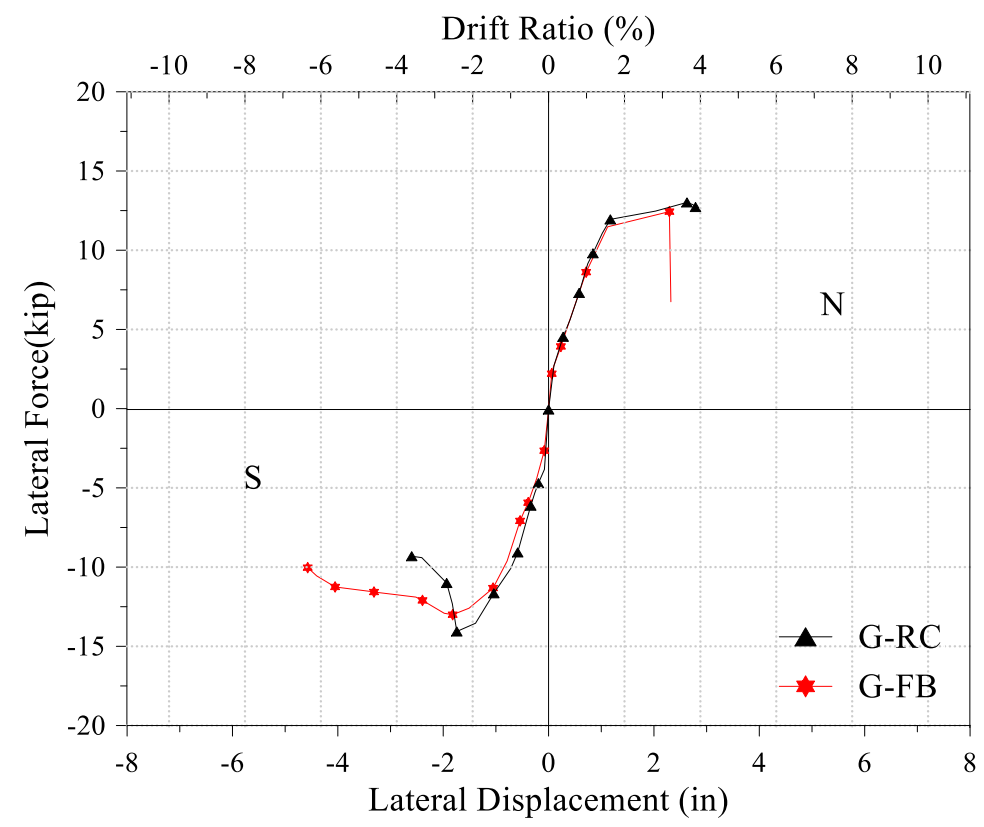

Figure 3.45 Backbone curves comparison of the new pattern SGICF walls. 


\subsection{Deformation Performance}

Three deformation parameters were considered to determine the behavior of the SGICF walls subjected to a time history ground motion excitations. The external and internal instrumentations were considered in the current evaluation. Five string pots were used to determine the deformation shapes of the walls at each horizontal core level, and up to eight LVDTs were employed to calculate the curvature of the walls. The strain gauges that were placed on the vertical cores reinforcement were used to determine the curvature values at the base of the walls and the deformation componenets.

\subsubsection{Wall Deformation Shapes}

The deformation shape for each SGICF wall is shown in Figure 3.46. The legend in the bottom of the figure shows the symbol and the magnitude of the scaled ground motion where the maximum displacement of the string pot was recorded. For instance, \pm 0.3 refers to the maximum recorded displacement in the north and the south directions of the specimen during the ground motion event that was scaled to 0.3 . The markers indicate the different heights at which displacement was measured, i.e., the location of each string pot. The vertical axis of the plot refers to the height from the bottom of the wall, which is representing the horizontal cores level where the string pots were attached as was shown in Figure 3.18 and Figure 3.21. 


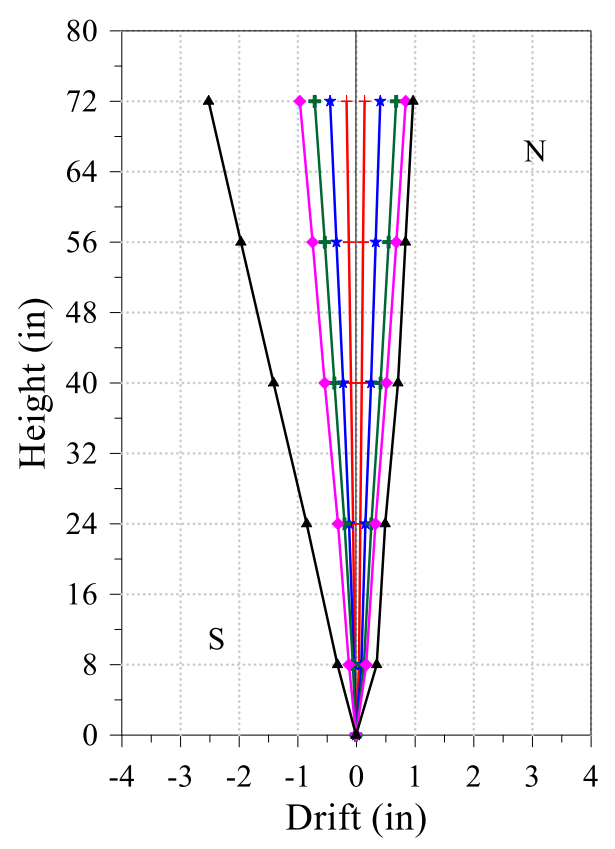

a) W-RC

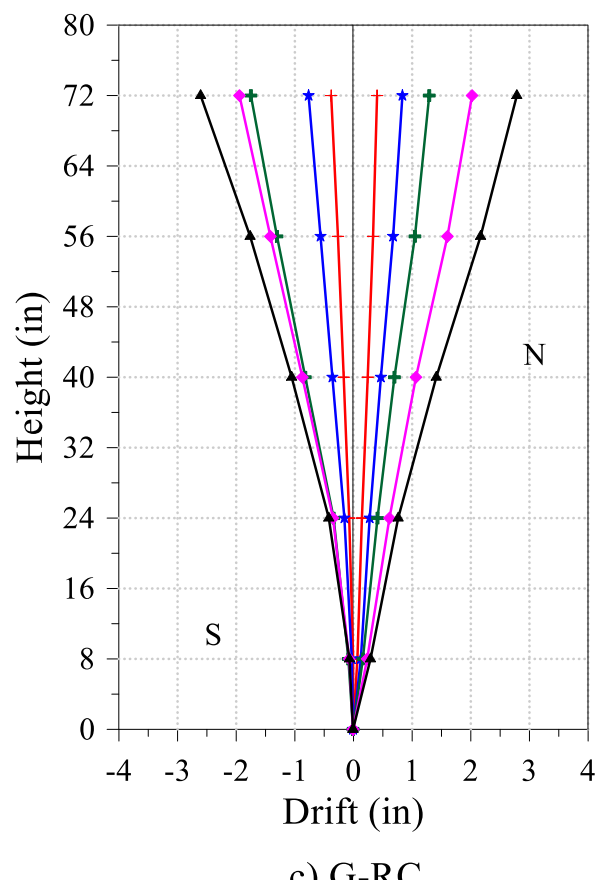

c) G-RC

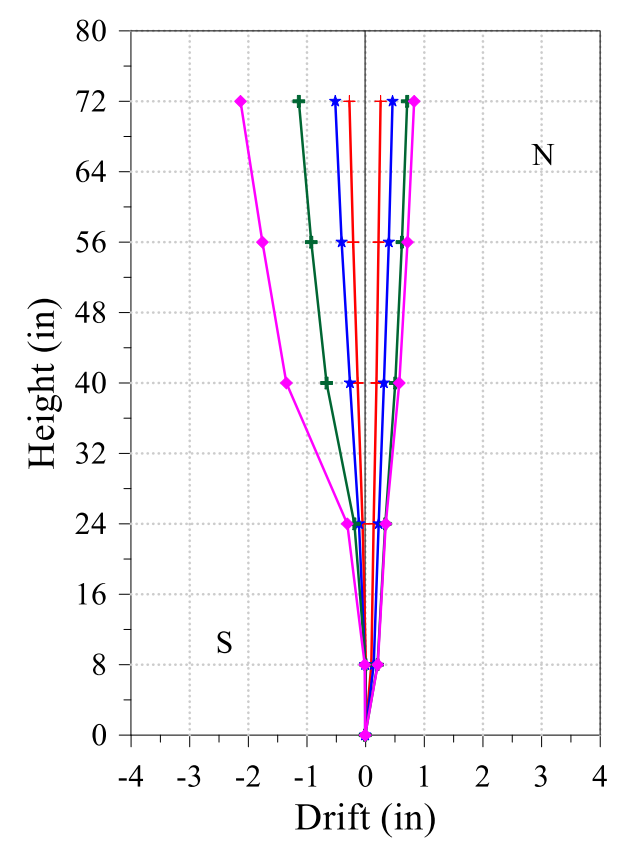

b) W-FB

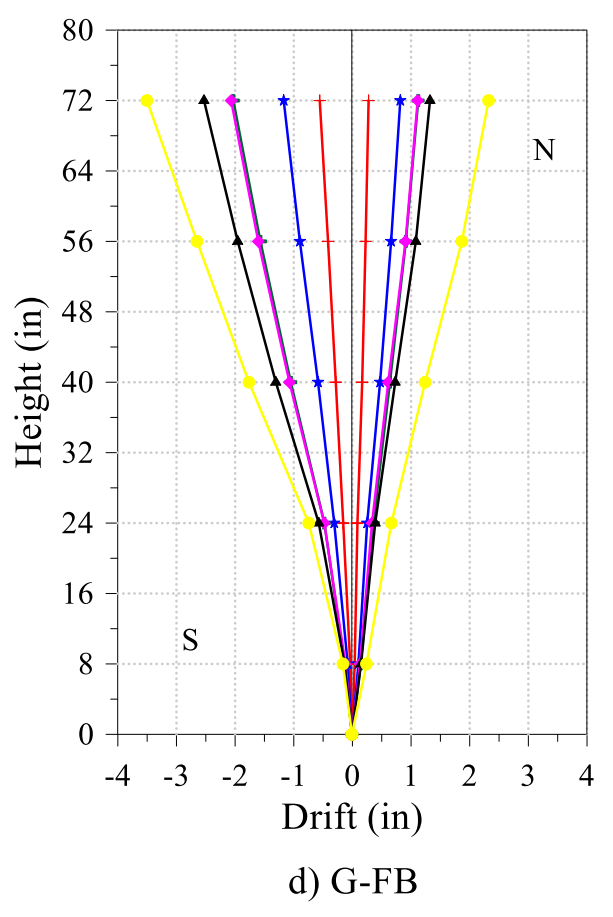

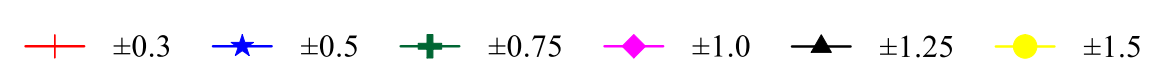

Figure 3.46 Deformation shapes for all tested SGICF walls. 
The deformation shapes for all SGICF walls were the same except W-FB wall, where voids were found between the second and the third level of the horizontal cores from the base of the wall. The deformation shape of $\mathrm{W}-\mathrm{RC}$ wall reveals a symmetric deformation behavior in both directions, especially during the low level of the applied ground motions. The high stiffness of the $\mathrm{W}-\mathrm{RC}$ wall, which was reported through the free vibration test was pronounced from the deformation shape as well. When the wall was subjected to SFGM of 1.25 , the values of the deformations increased and the collapse of the wall in the south direction was obvious in Figure 3.46a. Also, there was not a notable influence of the lap splice between the foundation dowels and the vertical cores reinforcement on the deformation shape of the $\mathrm{W}-\mathrm{RC}$ wall, which indicates that the bond between the concrete and the rebars was strong.

The influence of the voids found in the W-FB cores was not apparent after the application of the SFGM of 0.3, but its effect became obvious after the application of the SFGM of 0.75 especially in the south direction. The deformation shape of the W-FB wall was not symmetric and it showed large deformations above the second level of the horizontal cores.

The deformation shape of the G-RC wall showed a symmetric pattern in both directions, which indicates that the materials of the wall had similar quantities. As was shown previously, the G-RC wall experienced flexural cracks at the cores joints and the width of the cracks increased after the application of the high scaled ground motions. The increase in the flexural width cracks was caused by the hinging that occurred at the third 
level of the horizontal cores within the lap splice zone. The differential displacement measured in the deformation shapes confirmed the occurring of the hinging in this zone.

The deformation shape of the G-FB wall did not include the last 1.75 scaled ground motion because the wall was completely pulled out, and the reading of the string pots maxed out. The deformation shape of the G-FB wall showed that the wall experienced more deformation in the south direction than in the north. Also, the deformation shape indicated that the wall had a lower stiffness than the other walls, and that it tolerated more deformation capacity.

\subsubsection{Wall Curvature Profile}

The curvature of the SGICF walls was measured at different heights by using the vertical LVDTs that were mounted on both ends of the walls, and the strain gauges were placed on the outermost vertical steel bars near the base of the walls, as shown in Figure 3.17, Figure 3.18, Figure 3.20, and Figure 3.21. The curvatures in the SGICF walls were calculated based on the difference of the strain values between each pair of vertical LVDTs located respectively at the ends of the walls, divided by their horizontal distance. The strain value was calculated from the deformation reading of the LVDT divided by its strain gauge length. Curvature values for the base of the walls were measured from the reading of the strain gauges that were installed on the outermost vertical steel bars.

The curvature profile for each SGICF wall is shown in Figure 3.47. The legend in the bottom of the figure shows the symbol and the magnitude of the scaled ground 
motion where the maximum displacement of the string pot was recorded. To clarify, \pm 0.3 refers to the maximum recorded displacement in the north and the south directions of the specimen during the ground motion event that was scaled to 0.3 . Therefore, the curvatures of the walls were measured at the time when the maximum lateral displacement was recorded at each scaled ground motion. The markers indicated the different heights at which wall curvature was measured, i.e., the mid-distance between each consecutive LVDT. The vertical axis of the plot refers to the height of the wall from the base.

The curvature profile of $\mathrm{W}-\mathrm{RC}$ wall showed symmetric behavior with large curvature values at the base, where the moments were large and causing nonlinear deformation. The shape of the curvature profile indicates that the wall went through both shear and flexural deformations within the lap splice zone. On the other hand, the curvature profile of $\mathrm{W}-\mathrm{FB}$ walls was not symmetric, and the effect of cores voids was pronounced as shown in Figure 3.47b. Also, the irregularity of the curvature profile was within the voids reign between the second and fourth layer of the horizontal cores.

The curvature profiles of the new SGICF walls were symmetric, and the maximum curvature values were at the base. G-FB wall showed low curvature values above the lap splice zone, when compared to the G-RC wall results. This finding could be contributed to the influence of the steel fiber concrete that was used in the G-FB wall by reducing shear failure. 


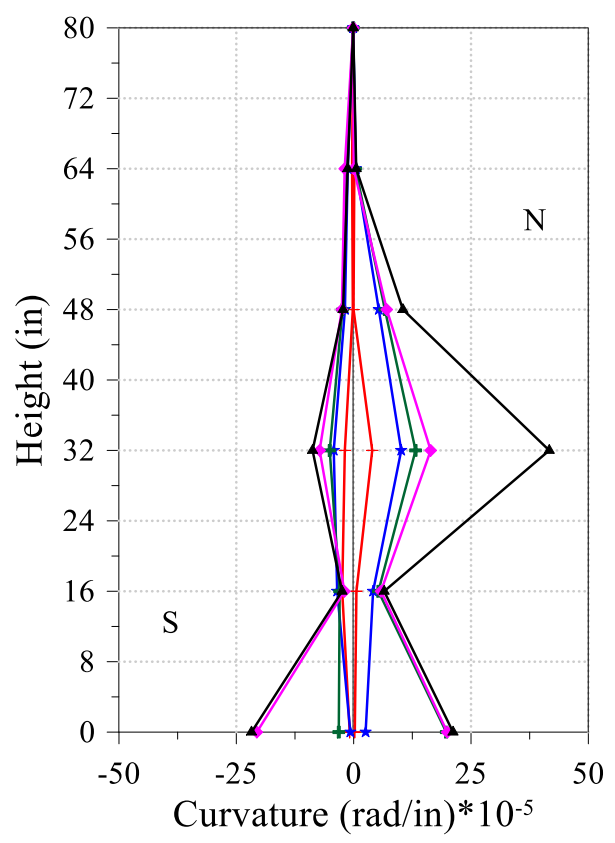

a) W-RC

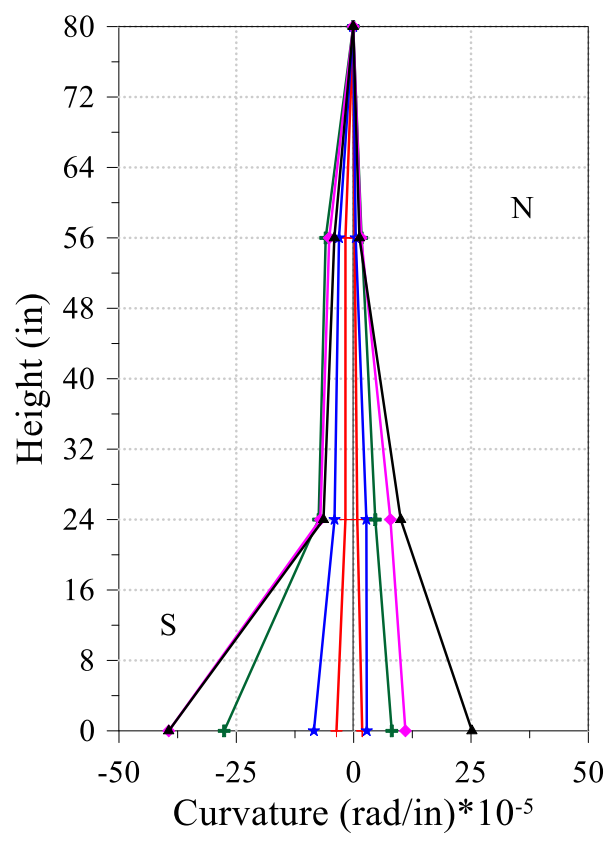

c) G-RC

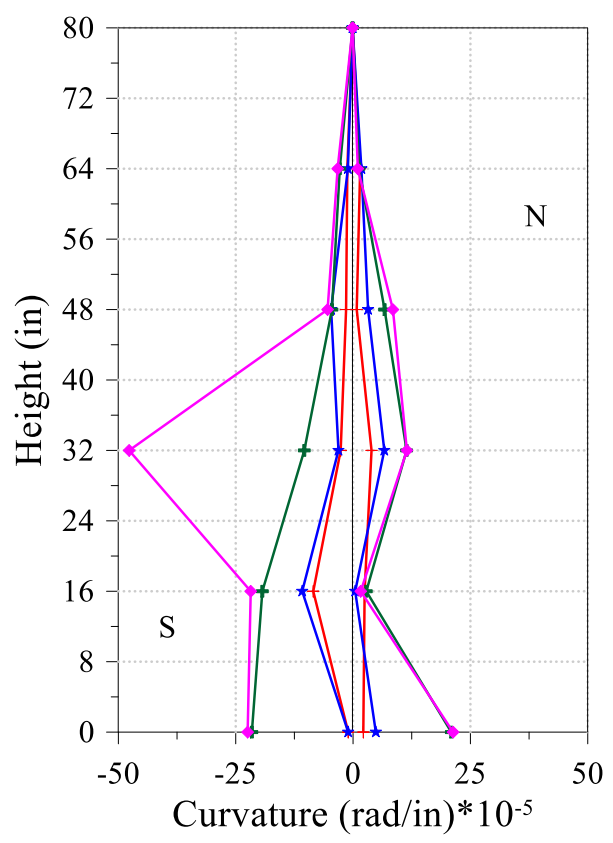

b) W-FB

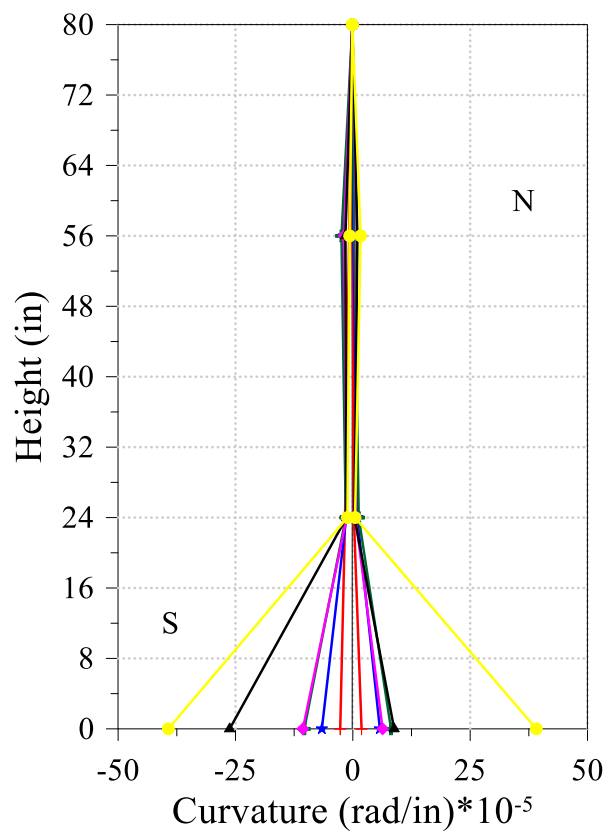

d) G-FB

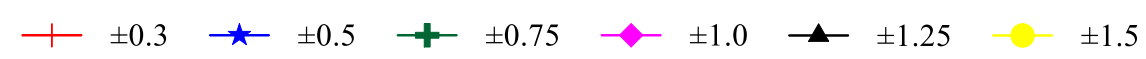

Figure 3.47 Curvature profile along the wall height for all tested SGICF walls. 


\subsubsection{Deformation components}

An attempt has been made to measure the influence of each deformation mode on the total displacement of the SGICF wall specimens by separately calculating the flexural deformation, shear deformation, horizontal sliding at the base, and longitudinal-bar slip at the base. The total lateral displacement $\Delta_{\mathrm{T}}$ can be divided into four components:

$$
\Delta_{\mathrm{T}}=\Delta_{f}+\Delta_{s}+\Delta_{h s}+\Delta_{b s}
$$

Where, $\Delta_{f}, \Delta_{s}, \Delta_{h s}$, and $\Delta_{b s}$ are the lateral displacements related to flexural behavior, shear, horizontal sliding at the base, and to the longitudinal-bar slip at the base of the wall, respectively.

Since the suggested instrumentation did not count for the global shear of the SGICF walls, horizontal sliding at the base, and longitudinal-bar slip at the base, the flexural deformation was decoupled from the other deformation components and the total lateral deformation equation became:

$$
\Delta_{\mathrm{T}}=\Delta_{f}+\Delta_{O D}
$$

Where $\triangle_{O D}$ is the representation of the lateral displacement due to shear, horizontal sliding, and longitudinal-bar slip at the base of the SGICF wall. The flexural deformation of the SGICF walls can be calculated as

$$
\Delta_{f}=\theta \mathrm{h}
$$

Where $\theta$ is the rotation over the height $\mathrm{h}$ where the lateral displacement was measured, which equals 
$\theta=\frac{V_{L}-V_{R}}{L}$

Where. $V_{L}$ and $V_{R}$ are the vertical displacements along the SGICF wall edges, and $\mathrm{L}$ is the horizontal distance between the vertical LVDTs. Since the vertical LVDTs that were placed on each SGICF wall measure the vertical displacement from the center of the horizontal core close to the wall's base, the contribution of the vertical displacement from the base of the specimens was measured from the strain gauges that were placed on the dowels of the vertical reinforcements.

The deformation component ratio for each SGICF wall is shown in Figure 3.48. The legend in the bottom of the figure shows the type of the deformation component and the direction where the deformation was measured. The deformation contribution was measured at the time when the maximum lateral displacement was recorded at each scaled ground motion and for both directions (North and South).

The contribution of each mode varied with the degree of the applied scaled ground motion as shown in Figure 3.48. For instance, in wall specimen W-RC, the flexural deformations account for about an average of $25 \%$ of the total displacement during the 0.3 SFGM. The flexural deformations contribution started to increase and reached an average ratio of $35 \%$ at the end of the 0.75 SFGM. Later, the contribution of the flexural deformations started to decrease rapidly, especially on the south side of the specimen where the inertia mass was located. Yielding of the vertical rebar contributed to lower the flexural deformations resisted by the wall. On the other hand, the contribution of the flexural deformations of specimen W-FB was smaller and did not exceed $25 \%$ 
when compared to W-RC. The voids found in W-FB specimen could contribute to the low contribution of the flexural deformations.

The flexural deformations of G-RC specimen were low when compared to the EPS SGICF walls, and did not exceed $19 \%$ of the total lateral displacements as it is shown in Figure 3.48c. This ratio was even lower for G-FB specimen with the maximum contribution of the flexural deformations of $9.25 \%$. The low contribution of the flexural deformations was also obvious on the curvature profile of G-FB specimen as was shown in Figure 3.47d. One of the important observations of the failure modes of G-FB specimen that the concrete honeycomb found in some horizontal cores influenced the contribution of the flexural deformations and gave advantages to other deformations, especially shear to dominate the overall deformation.

\subsection{Summary of the Shake Table Tests}

In tests of full-scale SGICF walls under in-plane real-time history dynamic loading, all SGICF walls behaved in a ductile manner over a range of scaled ground motions with lateral drift ratio that exceeded $2 \%$. Steel fiber-reinforced concrete did not improve the lateral strength of the SGICF walls but improved their lateral drift capacity. The EPS SGICF walls showed higher initial stiffness and lateral strength when compared with the new pattern of the SGICF walls. Different failure modes were observed and the flexural deformations percent were higher in walls with EPS forms. The newly suggested 
pattern of the SGICF walls showed higher drift ratios than the EPS walls and the additional rebar that was placed in each vertical core increased walls stability.

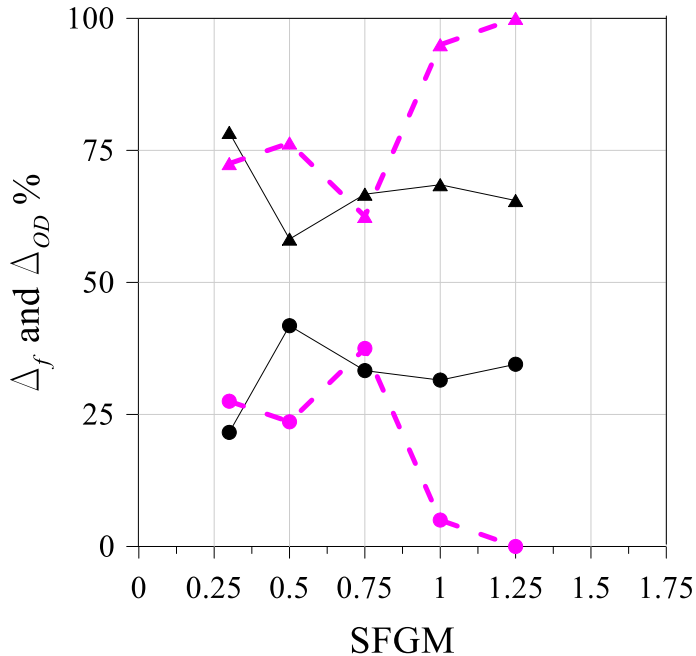

a) W-RC

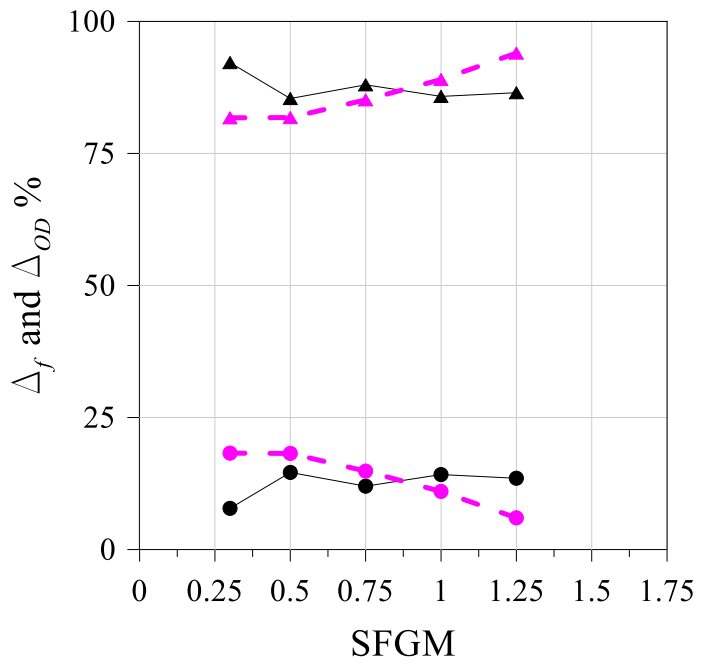

c) G-RC

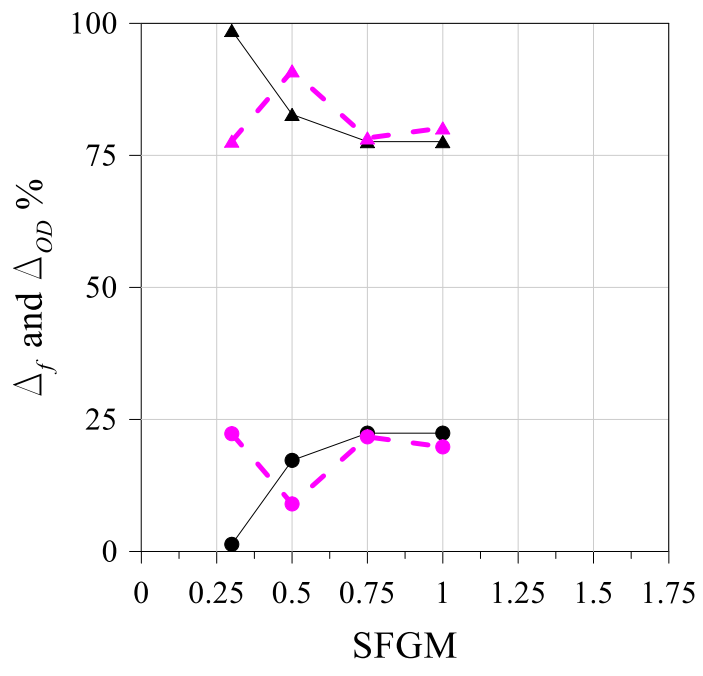

b) W-FB

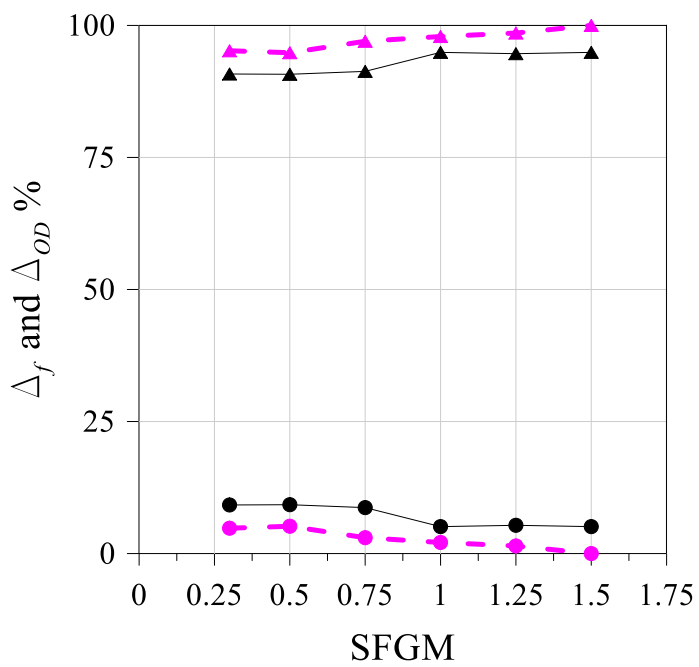

d) G-FB

- $\Delta_{f} \%$-North — $\Delta_{O D} \%$-North - - $\Delta_{f} \%$-South - $-\Delta_{O D} \%$-South

Figure 3.48 Deformation componenets contribution ratio. 


\section{CHAPTER 4: Component Level Cyclic Experimental Program}

\subsection{General}

Increasing concrete members' cross-sectional dimensions and using steel reinforcement stirrups were the typical ways to improve the shear resistance of any reinforced concrete structural member. Since the general practice of constructing ICF walls does not include using steel stirrups, and cores' cross-sectional dimensions are limited to the ICF blocks cores, using fiber reinforcement in the ICF walls became an alternative way to improve their shear capacity. Six small-scale circular reinforced concrete beams were prepared and tested to investigate the influence of Helix twisted micro-steel reinforcement on the shear capacity of the individual core of the two types of SGICF walls under cyclic loading. The moment-curvature relation of each beam was investigated. Beam specimens were divided into two sets; each set consisted of three specimens with different concrete types. For each set, reinforced concrete was assigned to one of the beams, and steel fiber-reinforced concrete allocated to the second and third specimens. This section first describes the specimen details, the experimental setup, the instrumentations used to monitor the state of circular beams during the cyclic loading, the loading protocol, and finally presents and discusses the results obtained.

\subsection{Specimen Details}

All of the circular beams in this investigation were 36 in. length. During the course of casting the SGICF walls, forms were made to cast six specimens to match the 
same concrete properties used in each wall. Also, each beam had a \#4 reinforcing bar, chosen from the same steel mill that was used for the vertical and horizontal core. Figure 4.1 shows the construction stages of the beam specimens. The rebar was placed on the center of each beam to simulate the same position of the reinforcement in the horizontal core of each SGICF wall.

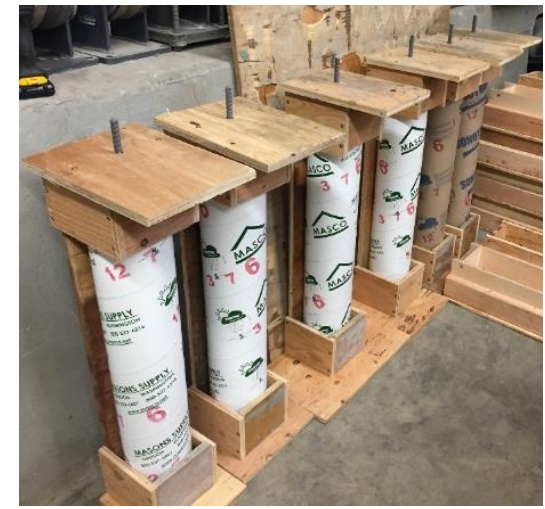

Beams forms

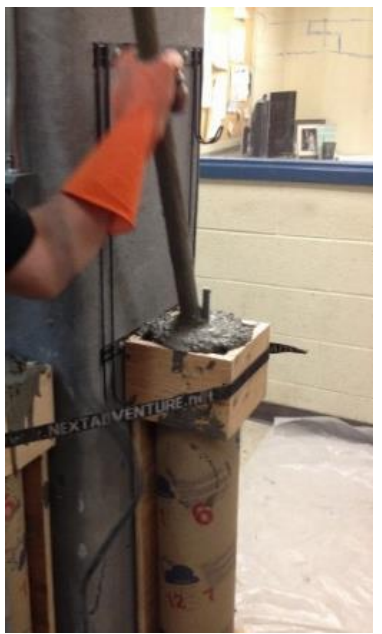

Concrete casting

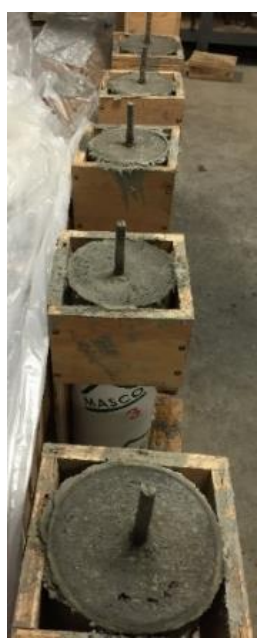

Specimens after curing

Figure 4.1 Construction of the beam specimens. 


\subsection{Test Setup and Procedure}

The six reinforced, simply supported concrete beams were tested by applying uniform line load of 6 in. on center. Each beam span length was selected based on the consideration of the half-length of the horizontal core on each side of the beam plus the diameter of the vertical core in the middle. For illustration, the span length of the first set of the beams was 22 in., which was based on half-length of the horizontal cores 8 in. on each side plus the 6 in. diameter of the vertical core obtained from the geometry details of the W-RC \& W-FB walls. The same approach was adopted for the second set of the beams with a total span length of $30 \mathrm{in}$. based on $12 \mathrm{in}$. half length of the horizontal cores on each side and 6 in. diameter of the vertical core that obtained from the geometry details of the G-RC \& G-FB walls. The beams were named as follow: specimens with span length of 22 in. were labeled as "S"(short) whereas specimens with a span length of 30 in. were labeled "L" (long), control specimens that have reinforced concrete were labeled "P0" whereas specimens that have steel fiber-reinforced concrete were labeled "F1" and "F2", respectively. Table 4-1 shows the test matrix and the designation for each beam test.

Table 4-1 Test matrix with beams designation.

\begin{tabular}{|c|c|c|c|}
\hline Designation & $\begin{array}{c}\text { Span Length } \\
\text { (in) }\end{array}$ & $\begin{array}{c}\text { Reinforcement } \\
\text { size }\end{array}$ & Concrete Type \\
\hline SP0 & 22 & $1-\# 4$ & Reinforced Concrete \\
\hline SF1 & 22 & $1-\# 4$ & Steel Fiber-Reinforced Concrete \\
\hline SF2 & 22 & $1-\# 4$ & Steel Fiber-Reinforced Concrete \\
\hline LP0 & 30 & $1-\# 4$ & Reinforced Concrete \\
\hline LF1 & 30 & $1-\# 4$ & Steel Fiber-Reinforced Concrete \\
\hline LF2 & 30 & $1-\# 4$ & Steel Fiber-Reinforced Concrete \\
\hline
\end{tabular}


The test setup consisted of two W12x72 beams that were vertically separated by four hollow structural section of different sizes to provide a clear spacing of $51 \mathrm{in}$. A double acting hydraulic ram (Magister Hydraulics model WCL 2x10) was used to apply the cyclic loading protocol. The specimens rested on movable supports that could accommodate the span length of the two beams sets. Both supports were designed in a way to make the rotation resistance of the supports equal to zero. The loading line consisted of two half-cylinder collars with a total length of six in.. Figure 4.2 shows the test setup with the specimen.

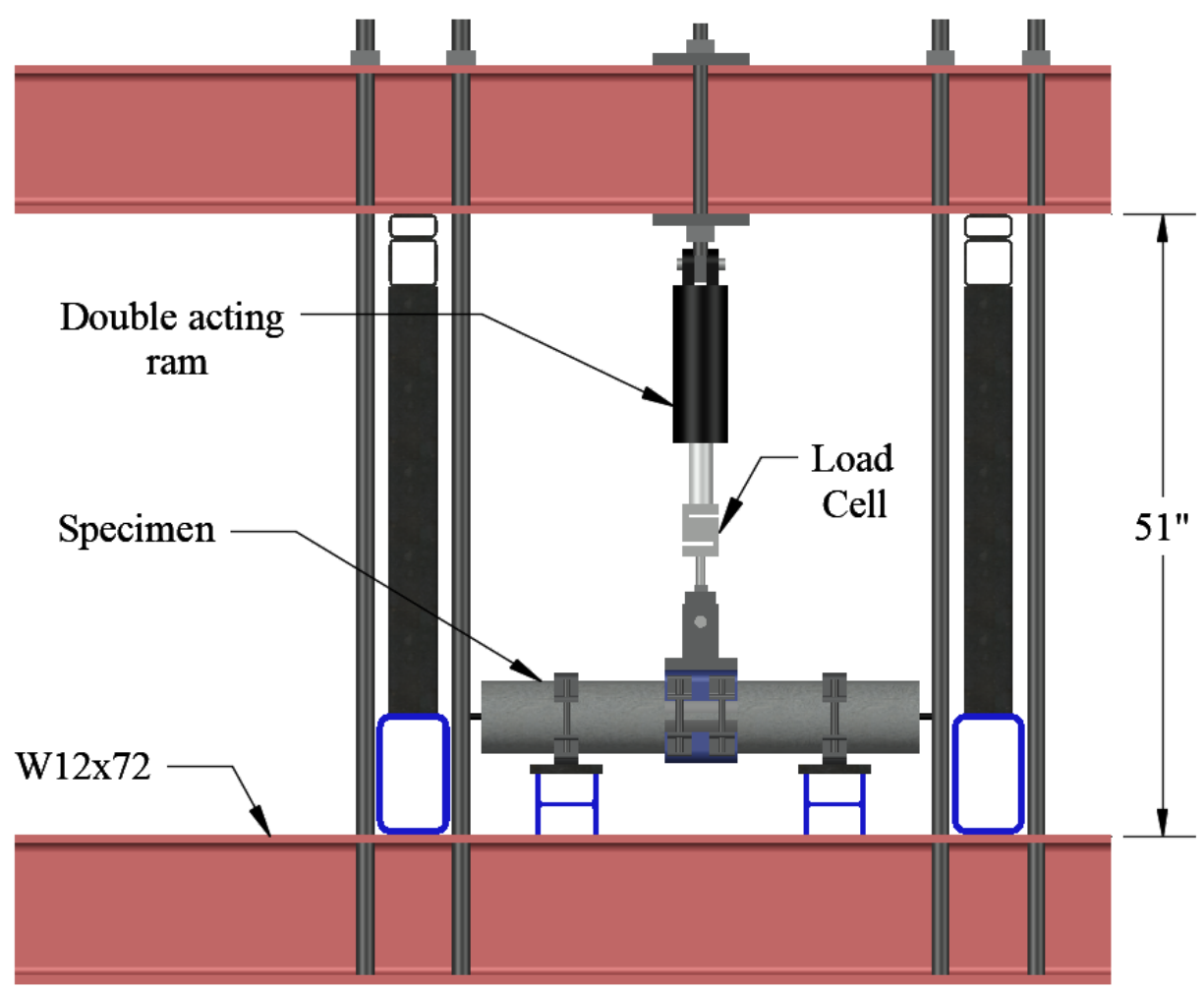

Figure 4.2 Beams test setup. 


\subsection{Instrumentations}

In order to analyze the behavior of the beams that were subjected to the cyclic loadings, the specimens were instrumented with different types of external instrumentations. The mid-span cyclic loading was measured by using OMEGA S-Beam load cell with a maximum force capacity of 20,000 pounds which was attached to the double acting ram as shown in Figure 4.2. Four LVDTs were used to measure the midspan deflection of each beam. LVDT1 and LVDT2 were attached to the supports for measuring the uplift displacement of the specimen during the uplift cycles as shown in Figure 4.3. LVDT3 and LVDT4 were used to measure the mid-span displacement. Therefore, the deflection of the beams was calculated by averaging the values of LVDT3\&4, and subtracted from the average values of LVDT1\&2.

Twelve position sensors were distributed at different sections of the beams to conclude the moment-curvature relationships. The distance between each position sensor was varied based on the span length of the beam as shown in Figure 4.3 and Figure 4.4. All six beams had the same instrumentations layout, except the first tested beam (LF1). The east side of the LF1 beam did not have the position sensor instrumentations. For beams with a span of 22 in., the total covered distance with the position sensors from the loading line was 4.5 in. on each side, whereas it was 8.5 in. for the beams with a span length of $30 \mathrm{in..}$ 


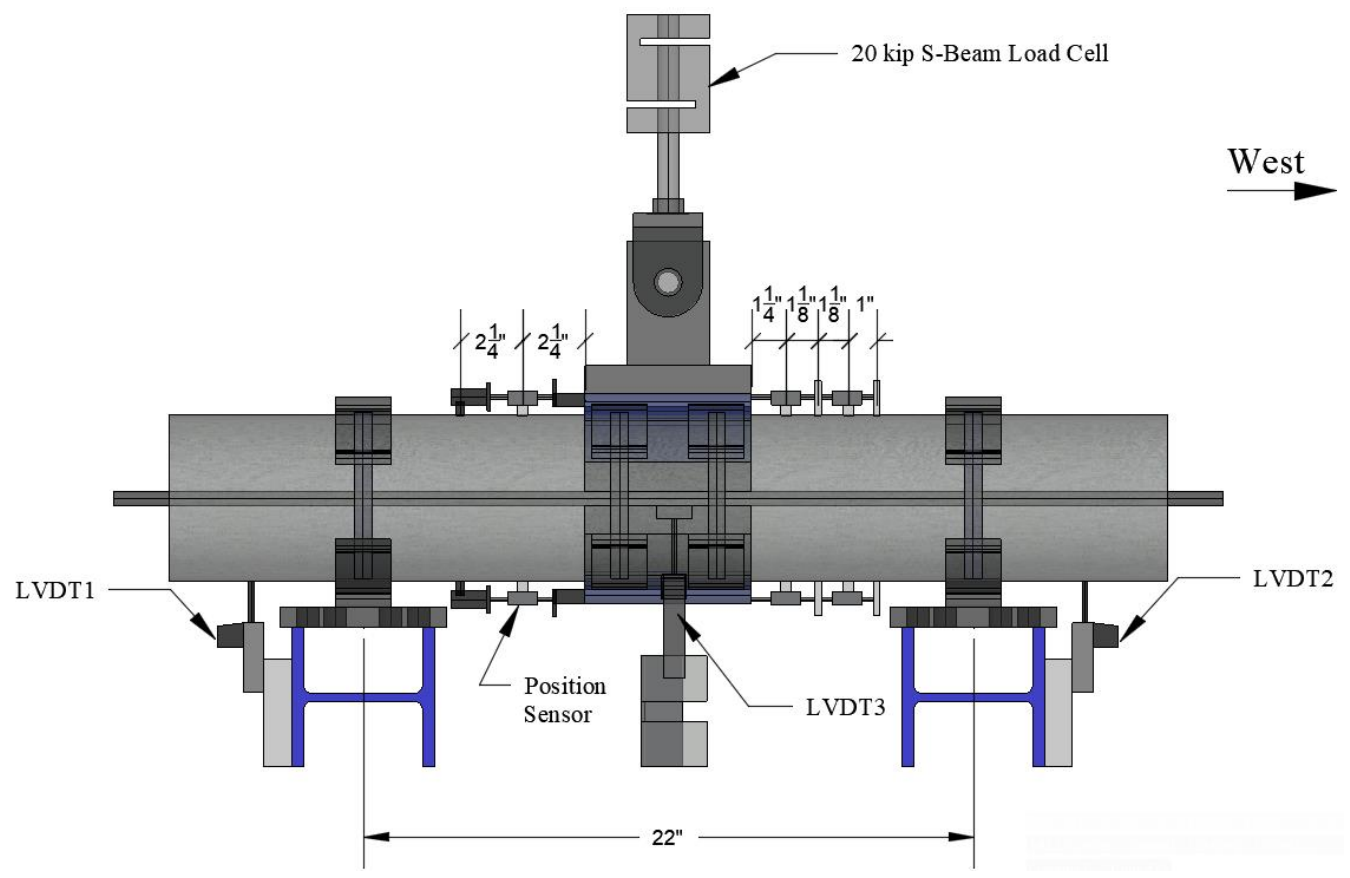

Figure 4.3 Instrumentation layout for set no. 1 beams.

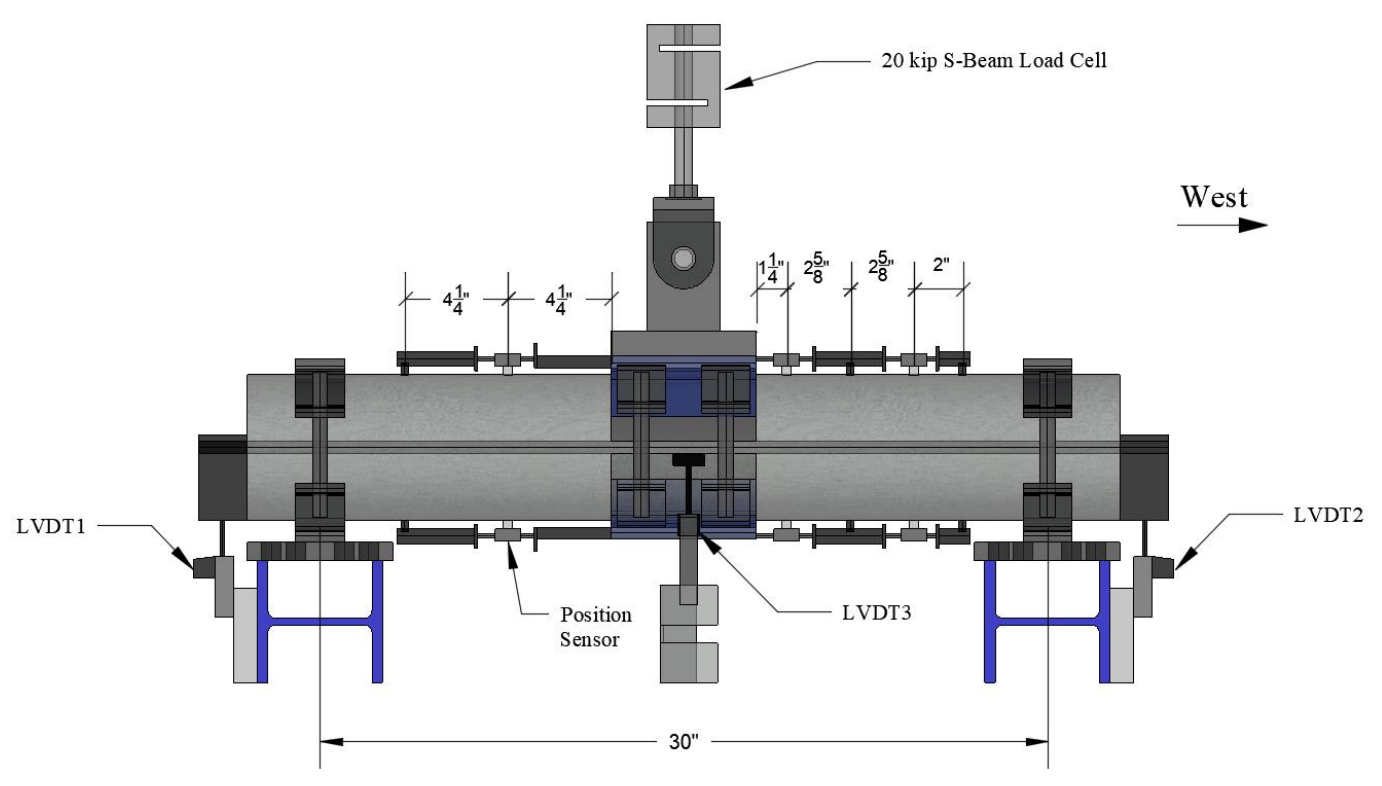

Figure 4.4 Instrumentation layout for set no. 2 beams. 


\subsection{Loading Protocol}

Force-control and displacement-control loading protocols were used in this experimental investigation. The force-control loading protocol was designed to capture the linear elastic behavior of the beams. One-quarter of the theoretical cracking moment load was selected as the start point of the force-control protocol, and the subsequent force values increased by a factor of 1.5 until the load of the full cracking moment was reached. The displacement-control loading protocol adopted in this investigation was similar to the loading protocol that was used for testing ICF walls under cyclic loading (Garth, 2014), and (Dusicka \& Kay, 2011). The displacement-control protocol was based on the recommendations by the ACI for testing unbonded post-tensioned precast shear walls (American Concrete Institute (ACI), 2008b) and moment frames (American Concrete Institute, 2001). The initial mid-span displacement was taken from the last cycle of the force-control loading protocol and then increased by a factor of 1.4. For each value of the loading protocols, three fully reversed cycles were applied.

Figure 4.5 shows the loading protocol used for the first set of the beams. The force-control loading protocol ended at cycle no. 12. The subsequent cycles were controlled by the displacement-loading protocol that started at a value of $0.005 \mathrm{in}$., and was increased by a factor of 1.4 after applying three fully reversed cycles. Figure 4.6 shows the loading protocol used for the second set of the beams. The same force-control loading protocol that used for the first set of the beams was applied to the second set of 
the beams. The displacement-control loading protocol of the second set of the beams was started with a value of 0.042 in..

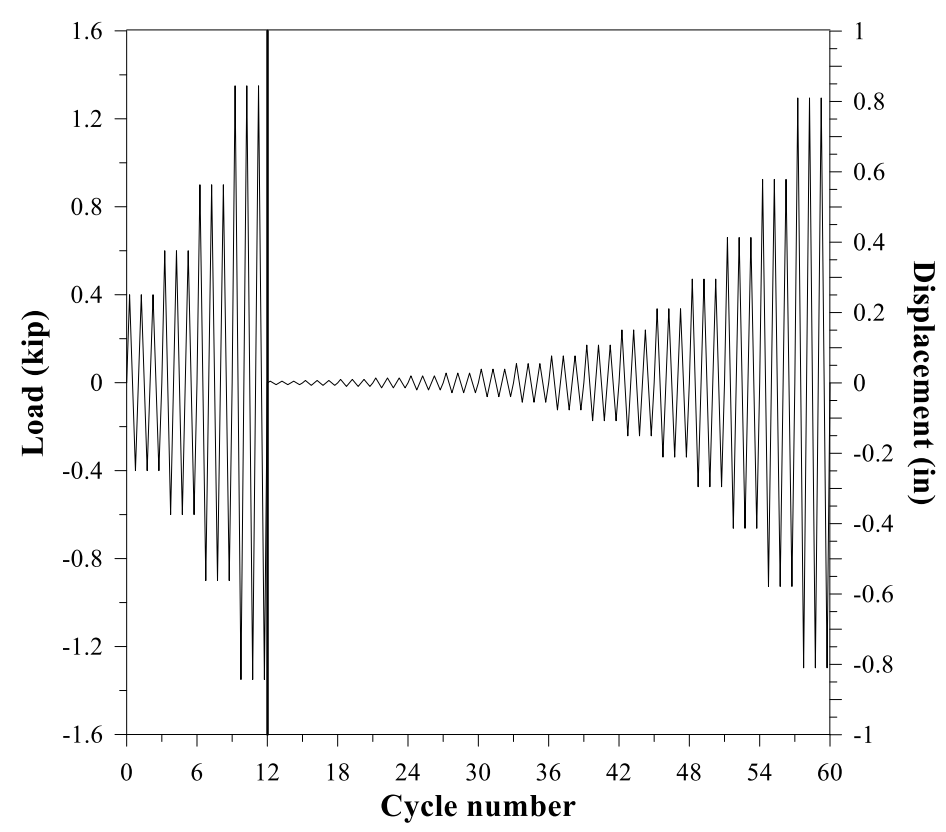

Figure 4.5 Loading protocol for the first set of the beams.

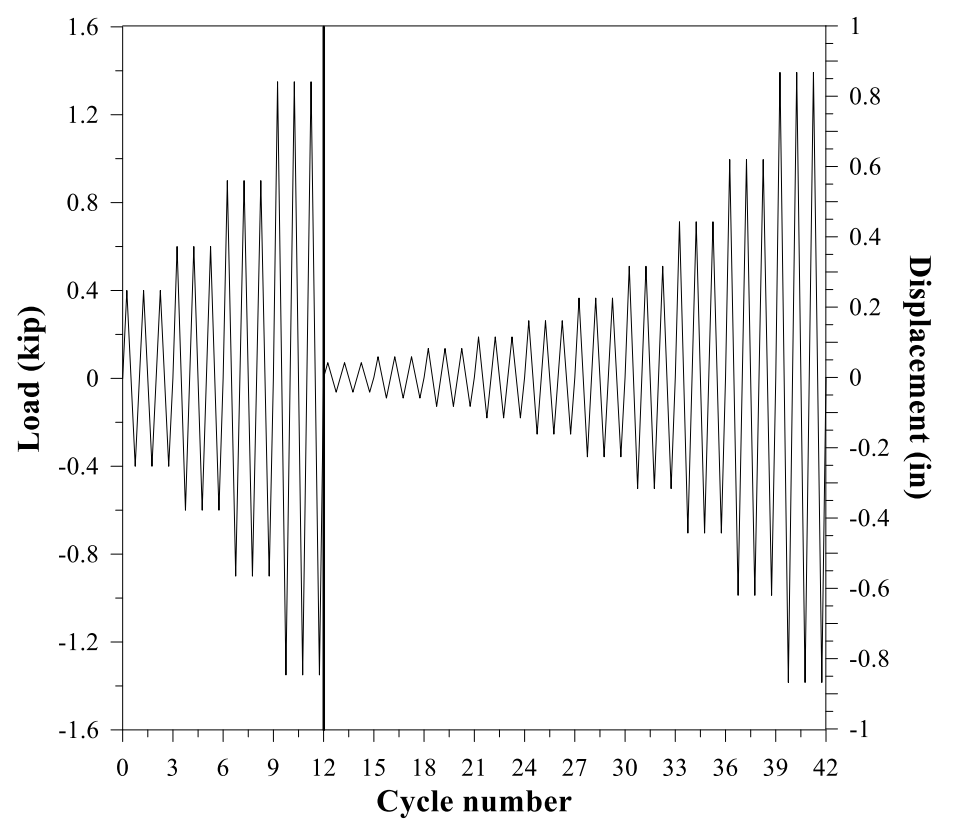

Figure 4.6 Loading protocol for the second set of the beams. 


\subsection{Failure Modes}

Each beam was subjected to the designed cyclic loading protocols and brought to failure. Observations of the generation of the flexural and shear cracks were made on each tested beam. The reduction in strength below $20 \%$ of the maximum strength of each beam was the guide to end the test.

\subsubsection{SP0 Specimen}

The application of the force-control loading protocol did not reveal any noticeable cracks on the SP0 specimen. The first flexural crack was observed when the deflection of the mid-span reached $0.18 \%$ drift (mid-span deflection/span length) close to the loading line as shown in Figure 4.7a. Later, several flexural cracks developed and extended around the loading line until the first shear crack generated on the right side of the specimen when the mid-span drift reached $0.96 \%$. The next major event occurred when the drift reached $2.64 \%$, where the shear cracks was extended from the loading line to the left side support as shown in Figure 4.7c. As the test continued, the width of both the flexural and shear cracks increased, and the specimen strength degraded until the specimen lost its integrity and collapsed at a drift of $3.72 \%$.

\subsubsection{SF1 Specimen}

Similarly to the SP0 specimen, the application of the force-control loading protocol did not disclose any clear cracks on the SF1 specimen. The first flexural crack was observed at the early stages of the displacement-control loading protocol when the 
deflection of the mid-span reached $0.02 \%$ drift, close to the right side of the loading line as shown in Figure 4.8a. When the drift of the mid-span reached $0.96 \%$, the shear crack started to generate on the back right side of the specimen as shown in Figure 4.8b. As the drift reached $1.85 \%$, shear cracks started to extend on both sides of the beam as shown in Figure $4.8 \mathrm{c}$. At a drift of $4.15 \%$, the concrete crushed around the loading line, the width of both the flexural and shear cracks increased, and the specimen strength degraded until the specimen lost its integrity and collapsed as shown in Figure 4.8d.

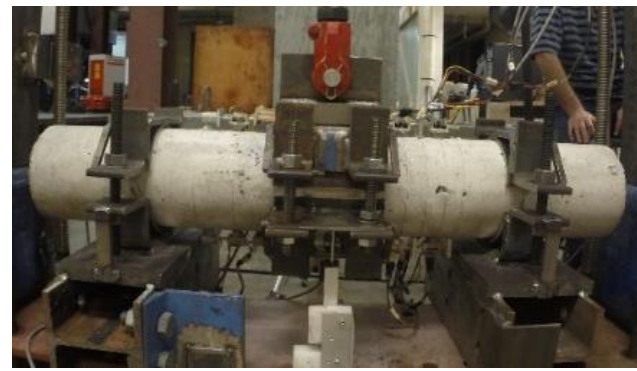

(a) $0.18 \%$ drift

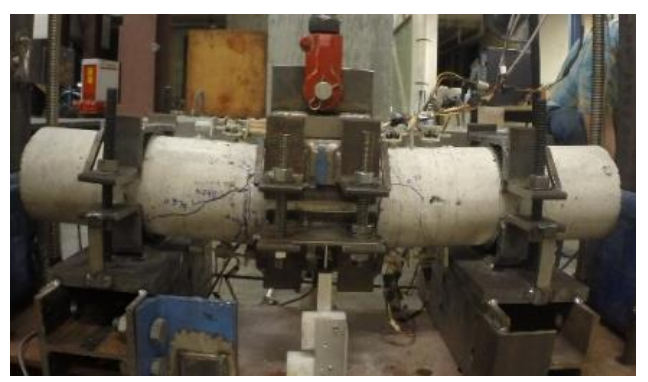

(c) $2.64 \%$ drift

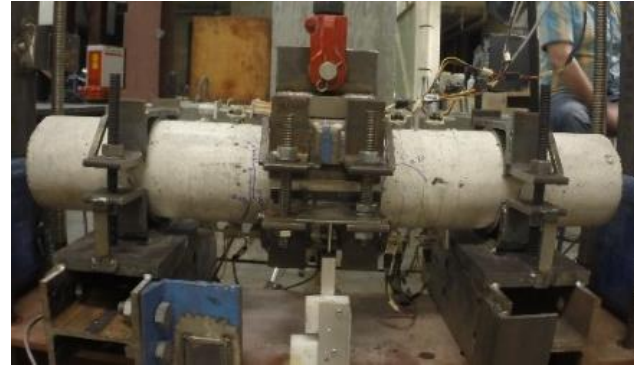

(b) $0.96 \%$ drift

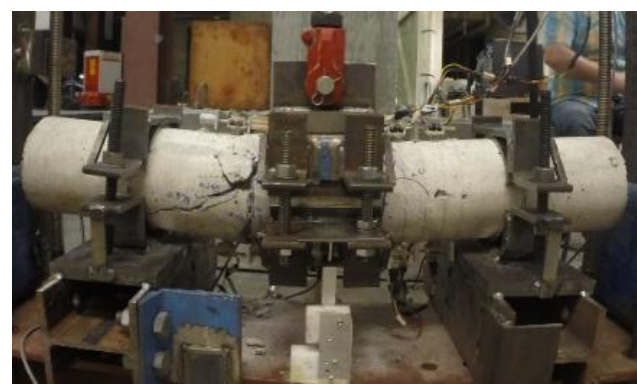

(d) $3.72 \%$ drift

Figure 4.7 Failure modes of SP0 specimen. 


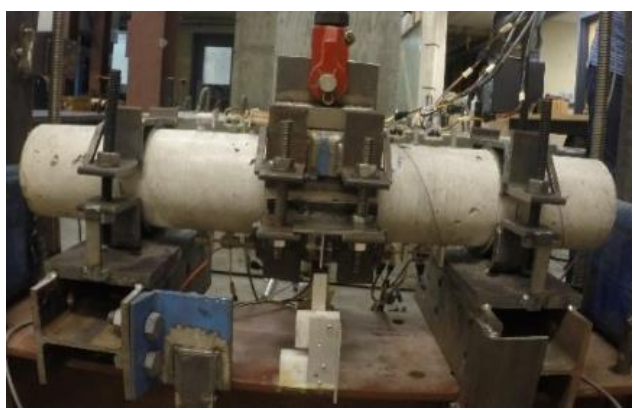

(a) $0.02 \%$ drift

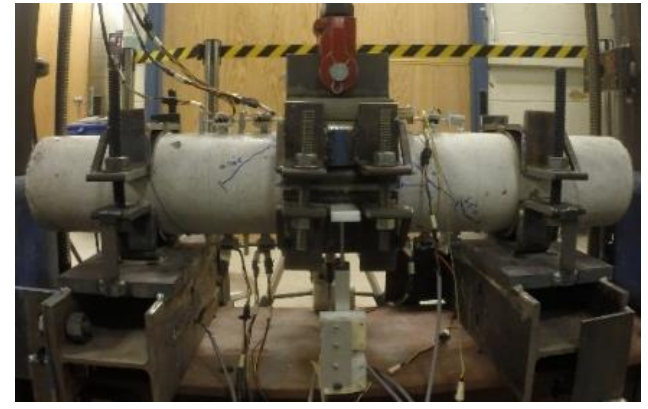

(c) $1.85 \%$ drift

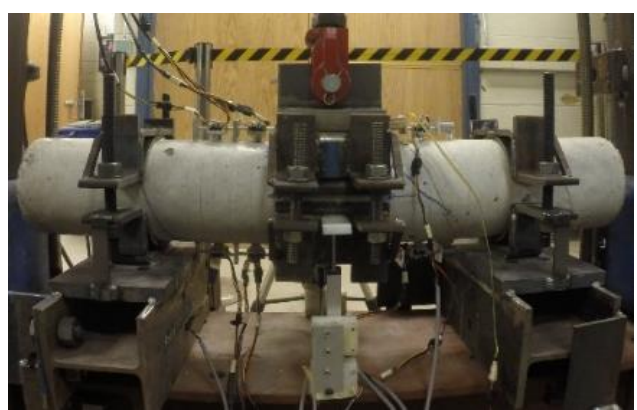

(b) $0.96 \%$ drift

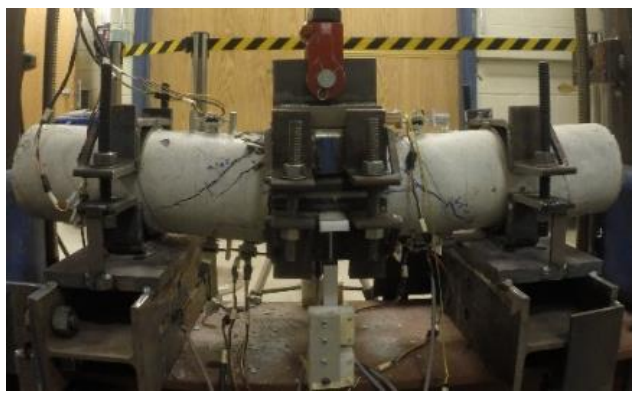

(d) $4.15 \%$

Figure 4.8 Failure modes of SF1 specimen.

\subsubsection{SF2 Specimen}

The SF2 specimen did not show any cracks during the force-control loading protocol. During the application and early stages of the displacement-control loading protocol, flexural cracks started to spread around the loading line when the drift ratio reached $0.023 \%$. After proceeding with the loading protocol, and when the drift ratio reached $0.69 \%$, a shear crack became apparent on the right side of the specimen starting from the loading line and extending towards the support as shown in Figure 4.9b. Later, shear failure became the dominant failure mode and shear cracks were spread on both sides of the loading line as shown in Figure 4.9c. As the test continued, the concrete 
spalled and crashed near the loading line, the width of both the flexural and shear cracks increased, and the specimen strength degraded until the specimen lost its integrity and collapsed at a drift of $3.73 \%$.

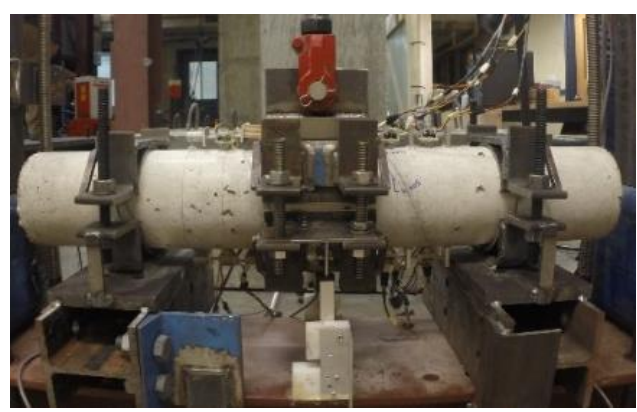

(a) $0.023 \%$ drift

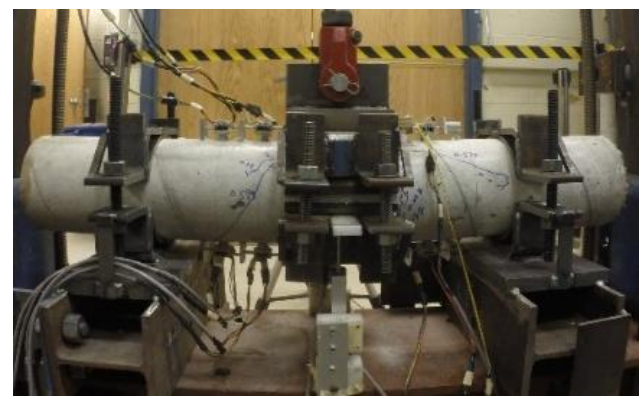

(c) $2.63 \%$ drift

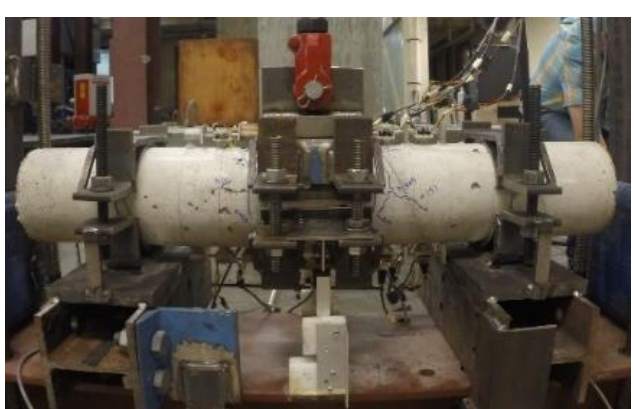

(b) $0.69 \%$ drift

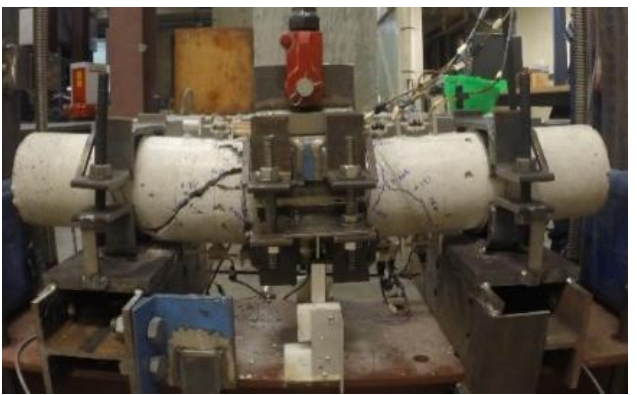

(d) $3.73 \%$

Figure 4.9 Failure modes of SF2 specimen.

\subsubsection{LP0 Specimen}

Figure 4.10 shows the failure modes of LP0 specimen. The first flexural crack happened when the mid-span drift reached $0.14 \%$ during the displacement-control loading protocol. After applying the subsequent loading cycles, flexural cracks around the loading line started to develop and the crack width of the flexural cracks increased. The shear cracks did not develop until the deflection of the mid-span reached 0.44 in 
when the first shear crack became obvious on the left side of the specimen as shown in Figure $4.10 \mathrm{~b}$. At a drift ratio of $2.9 \%$, shear cracks spread on both sides of the specimen but did not extend to the supports as shown in Figure 4.10c. The test was brought to an end when the flexural failure was dominant, and the degradation in the specimen strength was below $80 \%$.

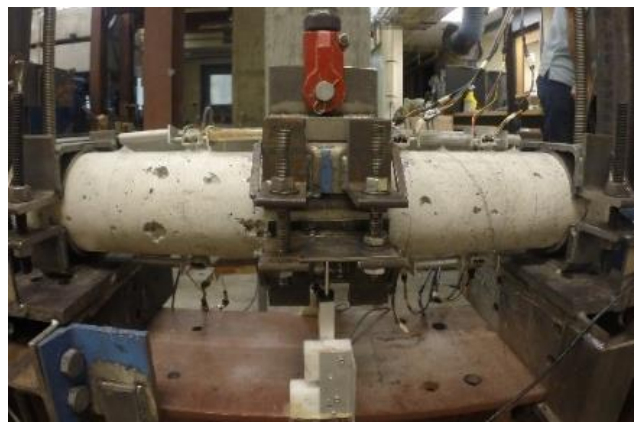

(a) $0.14 \%$ drift

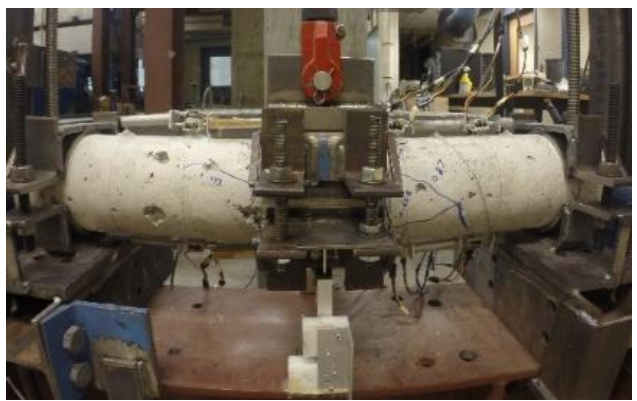

(c) $2.9 \%$ drift

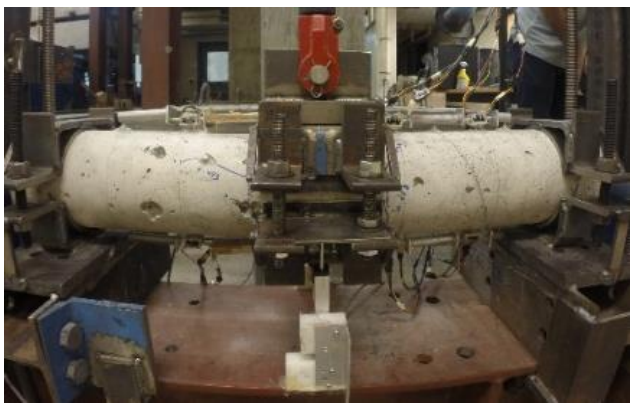

(b) $1.47 \%$ drift

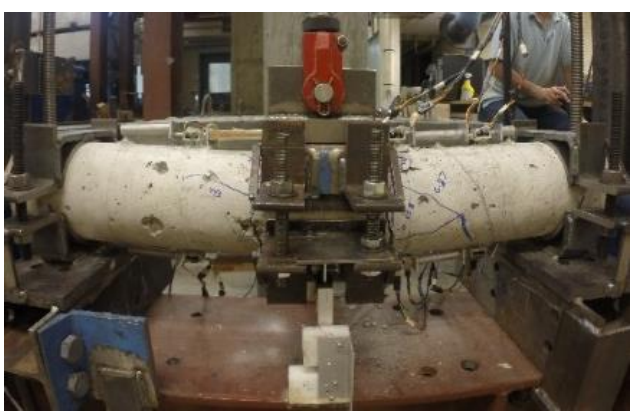

(d) $3.0 \%$ drift

Figure 4.10 Failure modes of LP0 specimen.

\subsubsection{LF1 Specimen}

Specimen LF1 was the first beam subjected to the cyclic loading, and the verification of the test setup was evaluated based on the specimen behavior. After assessing the failure modes, some changes had been recommended for the next tests by 
placing a $1 / 4$ inch diameter of steel rods under the specimen's ends before clamping the supports to ensure a simply supported beam action. As a result, failure modes of this specimen were not close to both LP0 and LF2 specimens.

Figure 4.11 shows the failure modes of the LF1 specimen. The application of the force-control loading protocol did not reveal any cracks on the LF1 specimen. The flexural cracks started to generate 2 in. away from the loading line, which was different from the behavior of LP0 specimen as shown in Figure 4.11a. At a drift ratio of 1.0\%, shear cracks became apparent on the right side of the specimen. The next major event occurred when the drift reached $2.25 \%$, where the shear cracks extended from the loading line to the right side support and caused a sudden drop in the specimen strength as shown in Figure 4.11d.

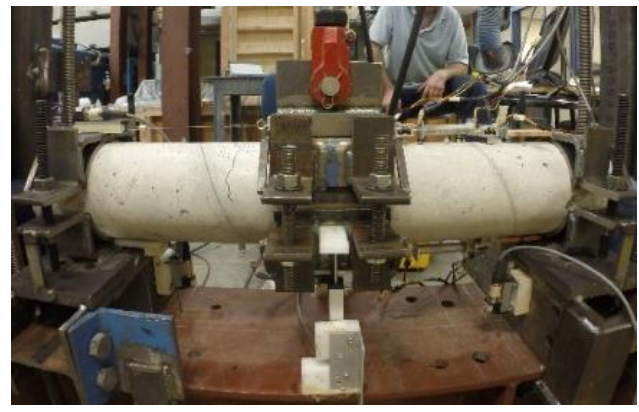

(a) $0.13 \%$ drift

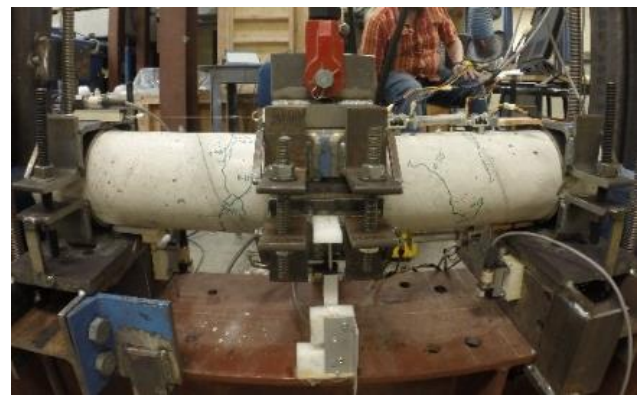

(c) $1.0 \% \mathrm{drift}$

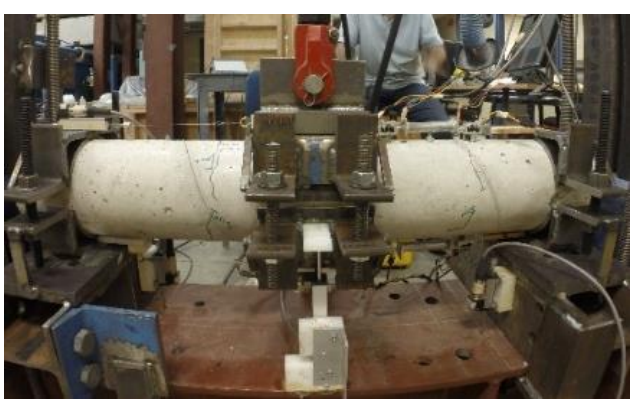

(b) $0.38 \%$ drift

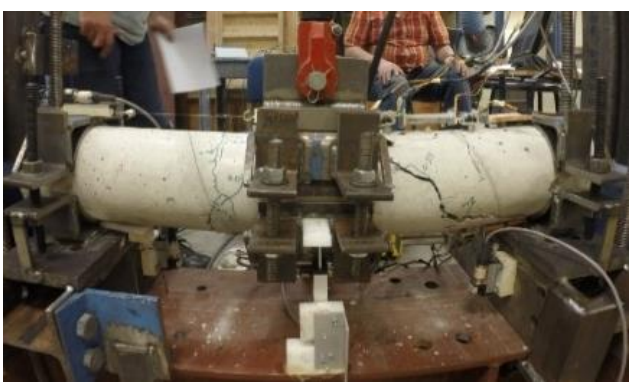

(d) $2.25 \%$ drift

Figure 4.11 Failure modes of LF1 specimen. 


\subsubsection{LF2 Specimen}

Figure 4.12 shows the failure modes of the LF2 specimen. Similarly to specimen LP0, the first flexural crack occurred when the mid-span drift reached $0.14 \%$ during the displacement-control loading protocol. At a drift ratio of $1.05 \%$, the concrete started to spall from the right bottom side of the beam as shown in Figure 4.12b. Also, the shear cracks generated at the same drift ratio (1.47\%) of LP0 specimen as shown in Figure 4.12c. The test was brought to an end when the flexural failure was dominant, and the degradation in the specimen strength was below $80 \%$.

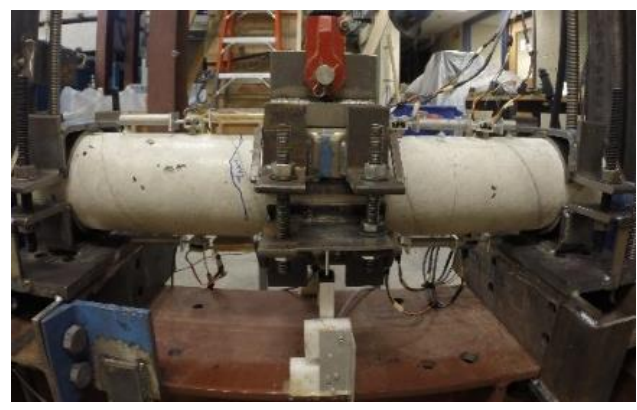

(a) $0.14 \%$ drift

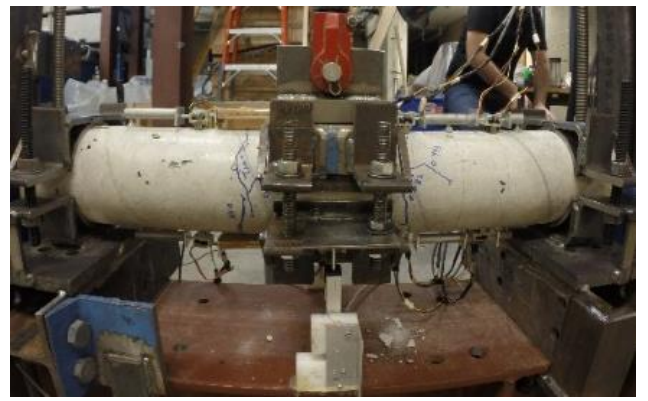

(c) $1.47 \%$ drift

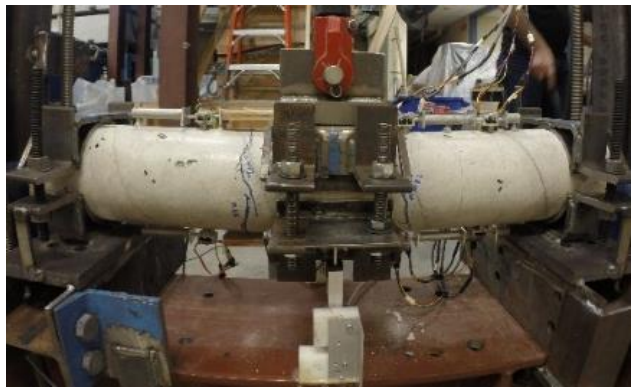

(b) $1.05 \%$ drift

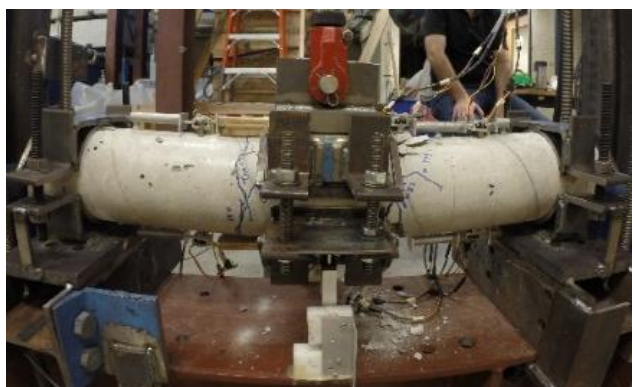

(d) $2.91 \% \mathrm{drift}$

Figure 4.12 Failure modes of LF2 specimen. 


\subsection{Test Results and Discussions}

Figure 4.13 shows the hysteresis curves of the first set of the beams. The maximum strength of all of the specimens revealed close values in both compression and tension directions. The hysteresis curve of the SF1 specimen flattened at a value of 11.7 kips because the lab view program limit was assigned to a lower value. After adjusting the lab view program limit, both SF2 and SP0 specimens showed close strength. Shear failure was dominant for all specimens, and there was not an apparent influence of the steel fibers on the failure modes of both SF1 and SF2 specimens. Also, the backbone curves agreement of the first set of the beams supports the fact that the steel fiber influence on the overall strength of the beams was minimal as shown in Figure 4.14.

The Helix steel fiber dose played a primary role in minimizing the expected improvement in the specimen's strength and failure modes because the same dose that was used for the SGICF walls specimens was used in these beams. Also, fiber length and the bond characteristics between the fibers and the concrete could be a reason for the lack of improvements in these specimens. 


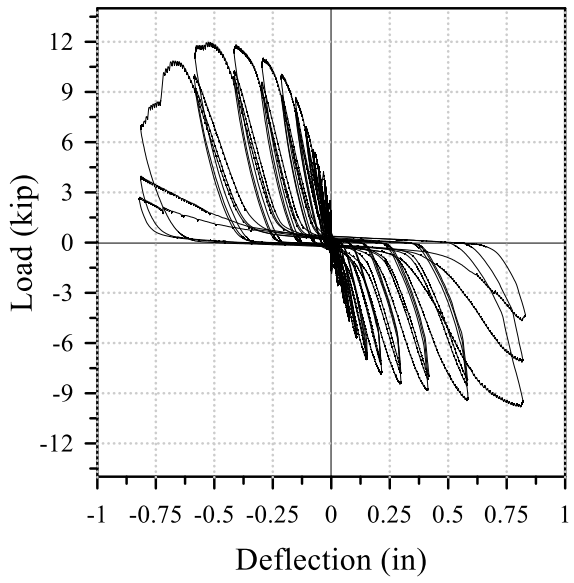

(a) SP0

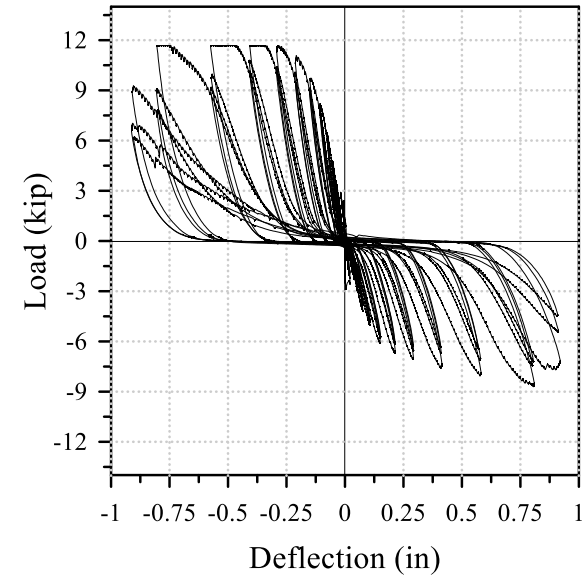

(b) SF1

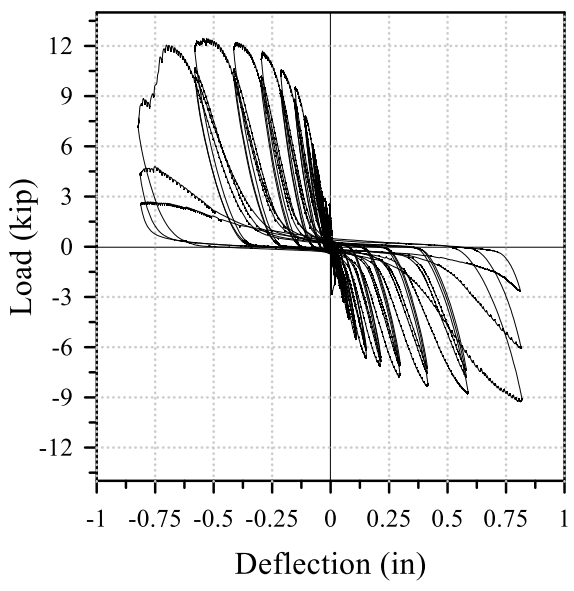

(c) SF2

Figure 4.13 Hysteresis curves for the first set of the beams. 


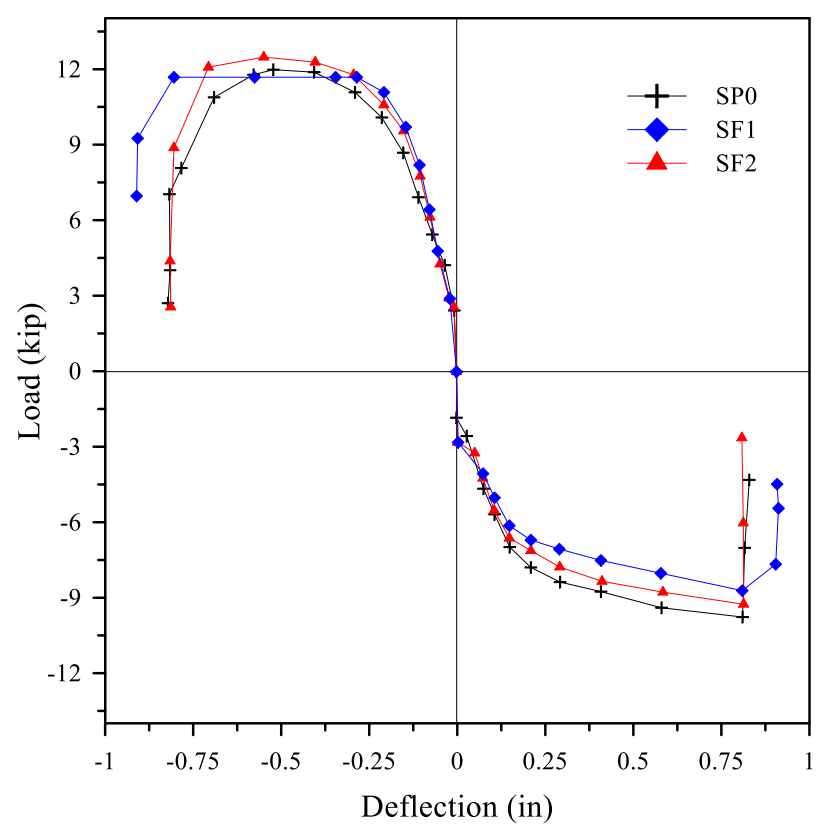

Figure 4.14 Load-displacement backbone curves for the first set of the beams.

Figure 4.15 and Figure 4.16 show the comparison between the moment-curvature backbones curves obtained from the position sensors. It was assumed that the plane section of the circular beams will remain plane after loading when measuring curvature at different loacations. It is worth mentioning that not all of the results obtained from the position sensors revealed a meaningful data, and only the results obtained from some of the position sensors that were placed close to the loading line provided acceptable data. The moment-curvature curves of SP0 and SF2 specimens extracted from the position sensors placed on the east side of the specimen did support the conclusions made previously about the steel fiber influence on the overall strength as shown in Figure 4.16. 


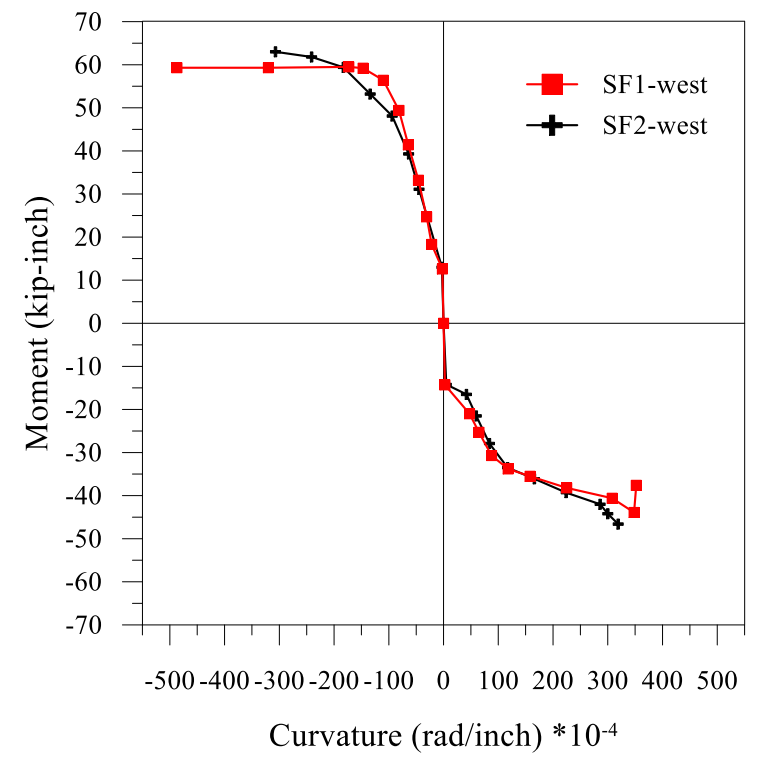

Figure 4.15 Moment curvature backbone curves for SF1 and SF2 specimens (west side).

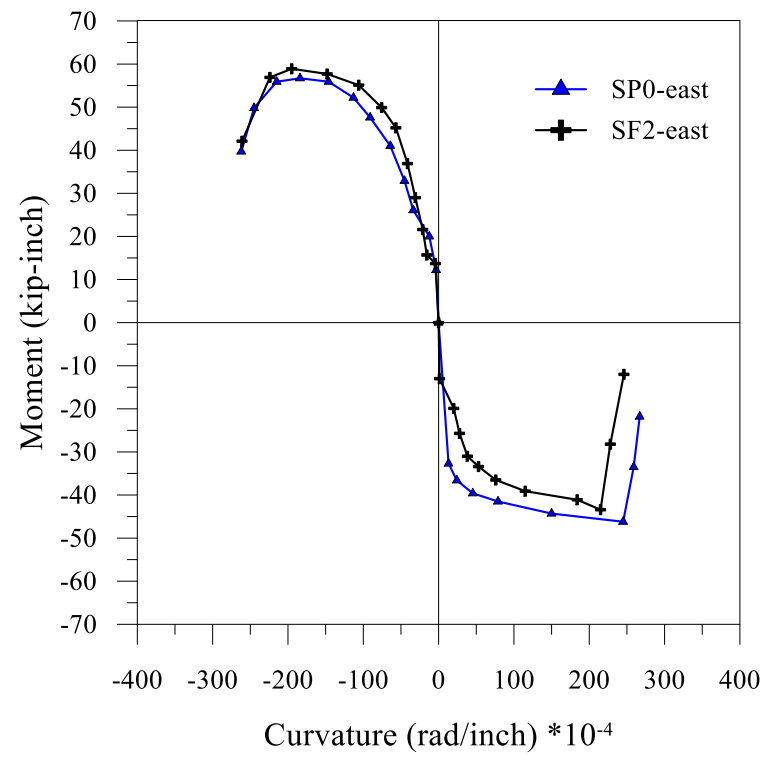

Figure 4.16 Moment curvature backbone curves for SP0 and SF2 specimens (east side). 
Figure 4.17 shows the hysteresis curves of the second set of the beams. The hysteresis curve of the LF1 specimen was different from the other specimens because the supports' modifications took place after the evaluation of the test setup was applied on this specimen. Flexural failure was dominant for both the LP0 and LF2 specimens, which supported the adopted design modification for G-RC and G-FB walls to reduce the shear demand on the horizontal cores. Also, the maximum strength of the LP0 and LF2 specimens revealed nearby values in both compression and tension directions.

The same conclusion that was made for the first set of the beams regarding the steel fiber influence on the beams' strength was applicable for the second set of the beams as well. Figure 4.18 shows similar behavior for both LP0 and LF2 specimens which led to the conclusion that the steel fibers did not improve the specimen's strength. Since the LF1 specimen was instrumented with the position sensors on the west side only, all of the position sensors did not provide a meaningful data. On the other hand, the position sensors that were mounted on both LP0 and LF2 specimens provided acceptable data. Figure 4.19 shows the moment-curvature backbone curves obtained for the position sensors, which were placed close to the loading line for both LP0 and LF2 specimens. The comparison between the moment-curvature curves supported the finding that the steel fibers did not show a remarkable effect on the specimen's strength. 


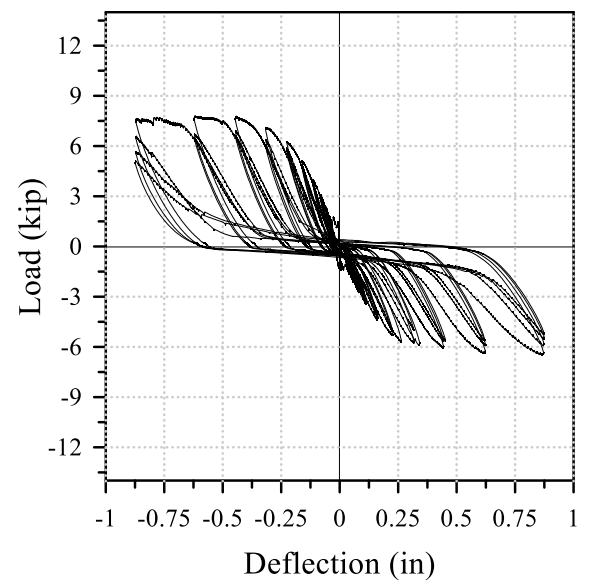

(a) LP0

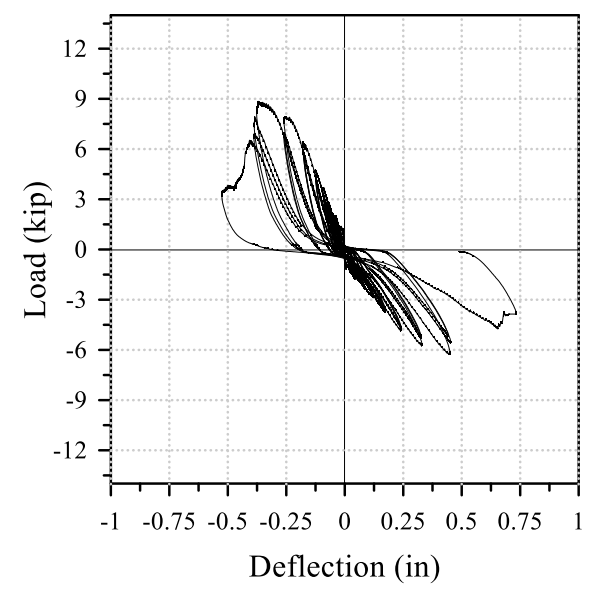

(b) LF1

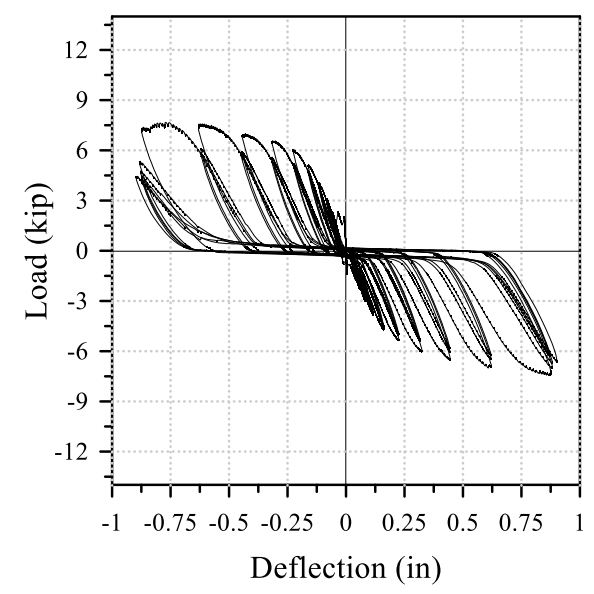

(c) LF2

Figure 4.17 Hysteresis curves for the second set of the beams. 


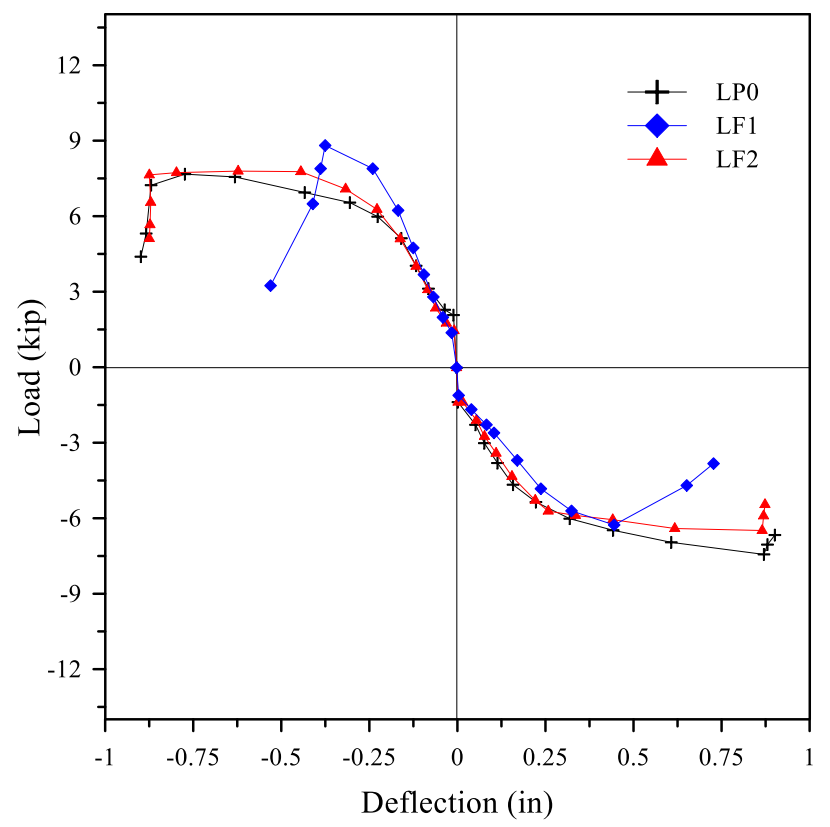

Figure 4.18 Load-displacement backbone curves for the second set of the beams.
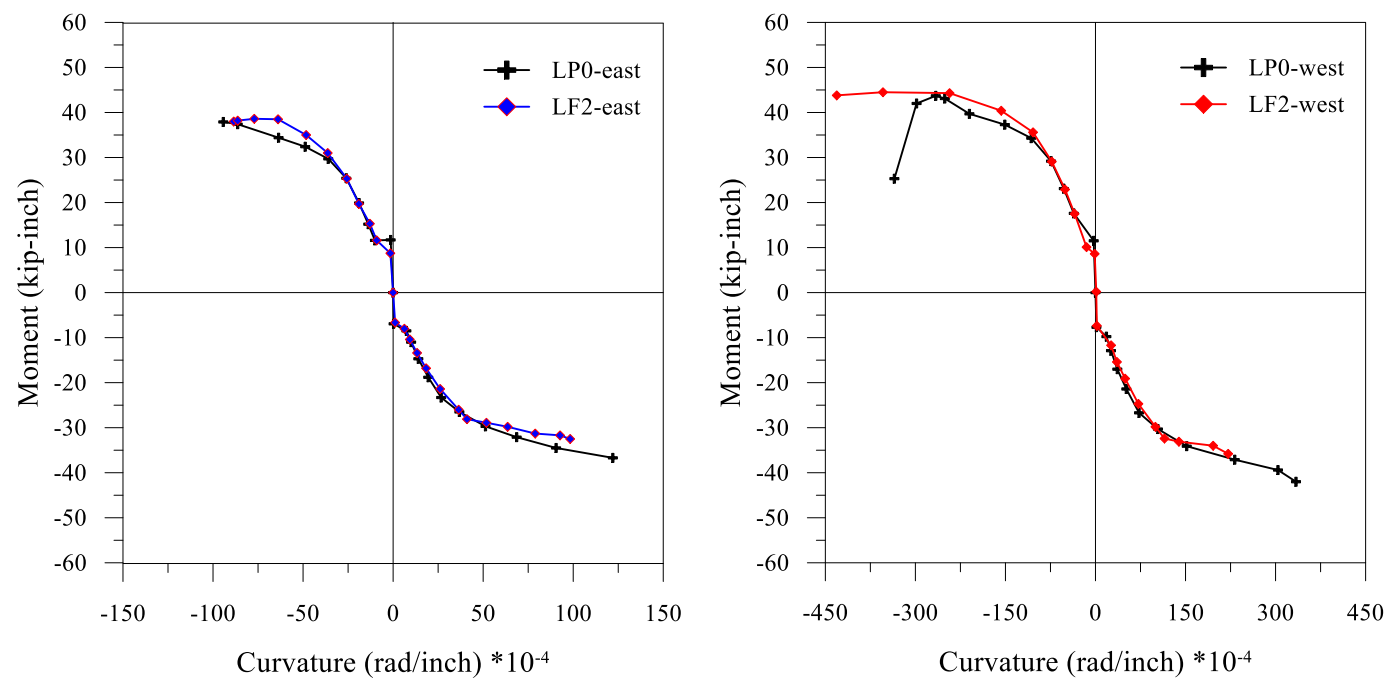

Figure 4.19 Moment curvature backbone curves for LP0 and LF2 specimens (east and west sides). 


\subsection{Summary of the Cyclic Tests}

After evaluating the influence of the steel fiber-reinforced concrete on the lateral strength and drift capacity of the SGICF walls by conducting shake table tests. A second experimental investigation was conducted at the component level of the SGICF walls for the same purpose. The influence of the steel fiber-reinforced concrete on the shear capacity of the individual core of the SGICF walls was experimentally investigated by testing six component level circular beams under cyclic loading. The specimens were selected to represent the horizontal cores of each type of the tested SGICF walls. The short span beams were representing the EPS SGICF walls, and the long span beams were representing the newly suggested pattern of the SGICF walls. The moment-curvature relation of each beam was investigated at different sections to study the influence of the twisted micro-steel reinforcement on the failure modes.

The cyclic tests results for both the short and long span beams provided a similar strength. The use of the steel fiber-reinforced concrete did not improve the strength and the deflection capacities of these specimens. This could be related to the fact that the Helix steel fiber dose played a primary role in affecting the expected improvement in the specimen's strength and failure modes. The shear failure mode was dominant for the

short span beams while flexural failure mode was dominant for the long span beam that has steel fiber-reinforced concrete. 


\section{CHAPTER 5: Evaluating Existing Analytical Estimations}

\subsection{General}

Different approaches researchers proposed to evaluate the initial stiffness and lateral strength of shear walls that have openings. An evaluation for the available analytical approaches has been investigated to estimate the initial stiffness and lateral strength of the SGICF walls, and to compare these with the obtained results from the experimental investigation. In order to simplify the analytical approaches, many assumptions were made to achieve this purpose and predict the most efficient approach.

\subsection{Estimation of the Initial Stiffness}

Due to the lack of experience of in running complicated and sophisticated finit element programs, one of the most efficient and rapid tools that many practicing engineers prefer to use in calculating the lateral stiffness of shear walls with openings is the simplified hand-calculated approach (Tena Colunga \& Rivera Hernández, 2015). Therefore, to estimate the SGICF wall's lateral intial stiffness, various methods were investigated to conclude the most efficient method that leads to a reasonable estimation. Since calculating the lateral intial stiffness of SGICF was the concern of this section, assumption to neglect the contribution of ICF forms was considered, linear-elastic behavior of the walls, no cracking, and the mesh of vertical and horizontal cores become the typical model for all methods. 


\subsubsection{Hand Calculation Approaches for Solid Shear Walls with Openings}

Several approaches were considered to estimate the lateral initial stiffness of shear walls with openings based on the methodology of calculating a solid shear wall's lateral displacement due to applying unit force at the top of the wall (Hsiao, 2014). For this purpose, two methods were presented with some assumptions about what is required to implement this methodology on the SGICF walls.

\subsubsection{Solid Shear Wall without Piers Rotation Effect (SOPR)}

The lateral displacement of the shear walls with openings was calculated by categorizing shear walls into three types of elements: solid walls, piers, and solid strips as shown in Figure 5.1. Consideration of cantilevered flexural deflection for the entire wall was made, while a fixed ended for both piers and solid strips with neglecting rotation effect of tops of the piers was considered. Moreover, this approach was meant to be used for one story walls. Therefore, an assumption was made to calculate the lateral displacement of the upcoming stories by considering cantilever flexural deflection for all shear wall elements. The below equations show the displacement calculation for a multistory shear wall with openings.

$$
\begin{array}{ll}
\Delta_{\text {moment }}=\frac{V h^{3}}{12 E I} & \text { For fixed-ended } \\
\Delta_{\text {moment }}=\frac{V h^{3}}{3 E I} & \text { For fixed-free-ended } \\
\Delta_{\text {shear }}=\frac{1.2 V h}{A G} &
\end{array}
$$




$$
\begin{aligned}
& \Delta_{\text {each element }}=\Delta_{\text {flextural }}+\Delta_{\text {shear }} \\
& \Delta_{\text {each story w/opening }}=\Delta_{\text {solid wall }}-\Delta_{\text {solid strip }}+\Delta_{\text {open strip }}
\end{aligned}
$$

Where:

$V=$ lateral load

$h=$ story height

$E=$ modulus of elasticity of the wall

$G=$ shear modulus of the wall

$A=$ cross-sectional area of each element

$I=$ moment of inertia of each element.

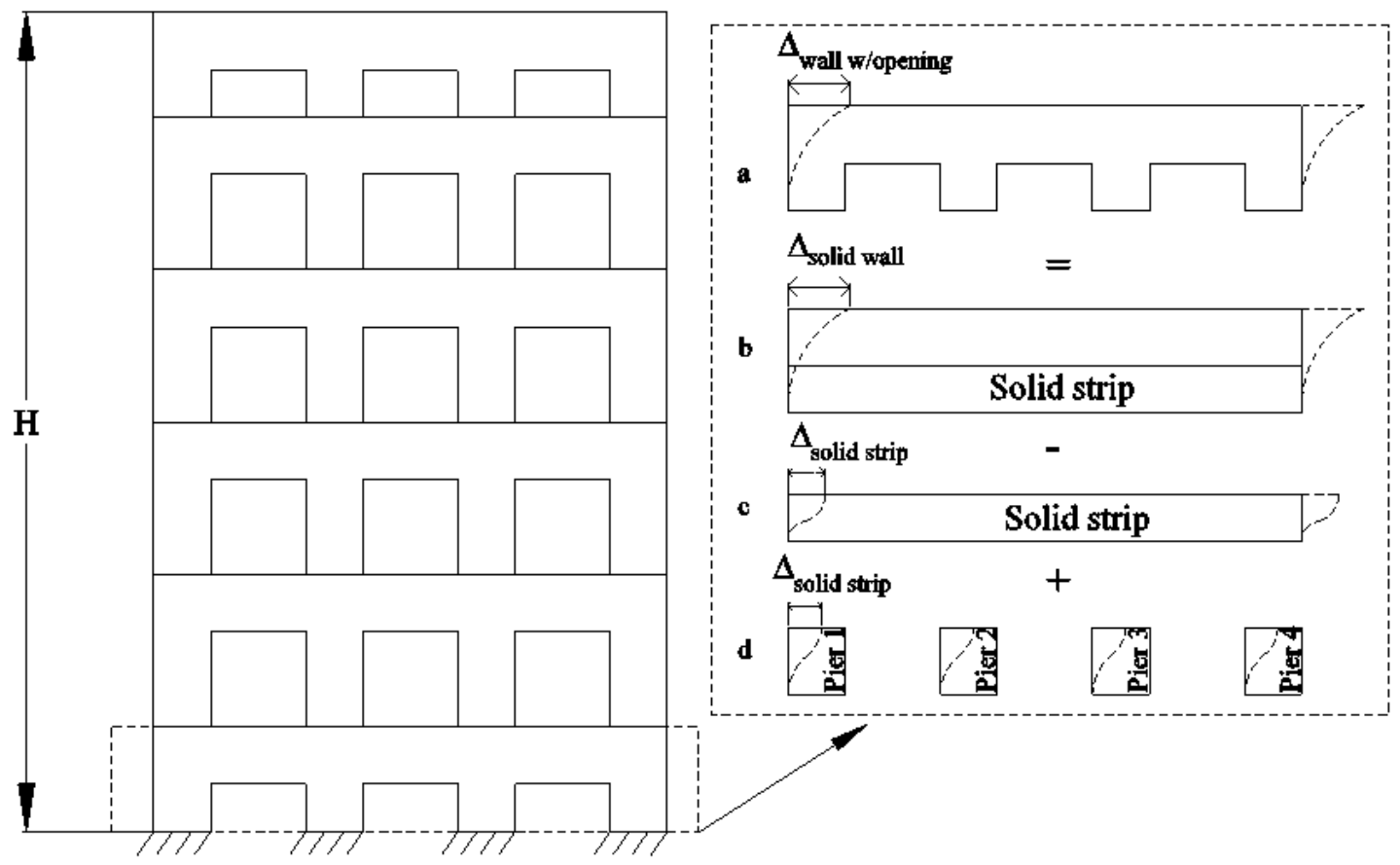

Figure 5.1 Solid shear wall without pier rotation effect. 


\subsubsection{Solid Shear Wall with Piers Rotation Effect (SWPR)}

The core difference between the SOPR and the SWPR methods is the rotation of the tops of the piers. Therefore, SGICF shear walls were modeled as a combination of beams and columns as shown in Figure 5.2. For this approach, the same calculation steps that were used for the SOPR were adopted except the rotation of piers' ends, which was calculated by using Eq. (5.6) for the first story, and Eq. (5.7) for the upcoming stories by considering a cantilever action for all of the SGICF wall's elements. Also, flexural displacements were computed for each element individually and lumped as a group, while shear displacement was calculated for the entire story and added to the total flexural displacement as shown in Eq. (5.8).

$$
\begin{gathered}
\Delta_{\text {pier of the first story }}=\frac{V\left(L_{c}\right)^{3}}{4 E I_{c}}\left(\frac{1}{3}+\frac{1}{6 K+1}\right) \\
\Delta_{\text {pier of upcoming stories }}=\frac{V\left(L_{c}\right)^{3}}{4 E I_{c}}\left(\frac{4}{3}+\frac{1}{6 K+1}\right) \\
\Delta_{\text {each story }}=\left(\Delta_{\text {solid wall }}-\Delta_{\text {solid strip }}+\Delta_{\text {open strip }}\right)_{\text {flextural }}+\Delta_{\text {shear }} \\
\text { Where: } \quad K=\frac{E I_{b} / L_{b}}{E I_{c} / L_{c}}
\end{gathered}
$$




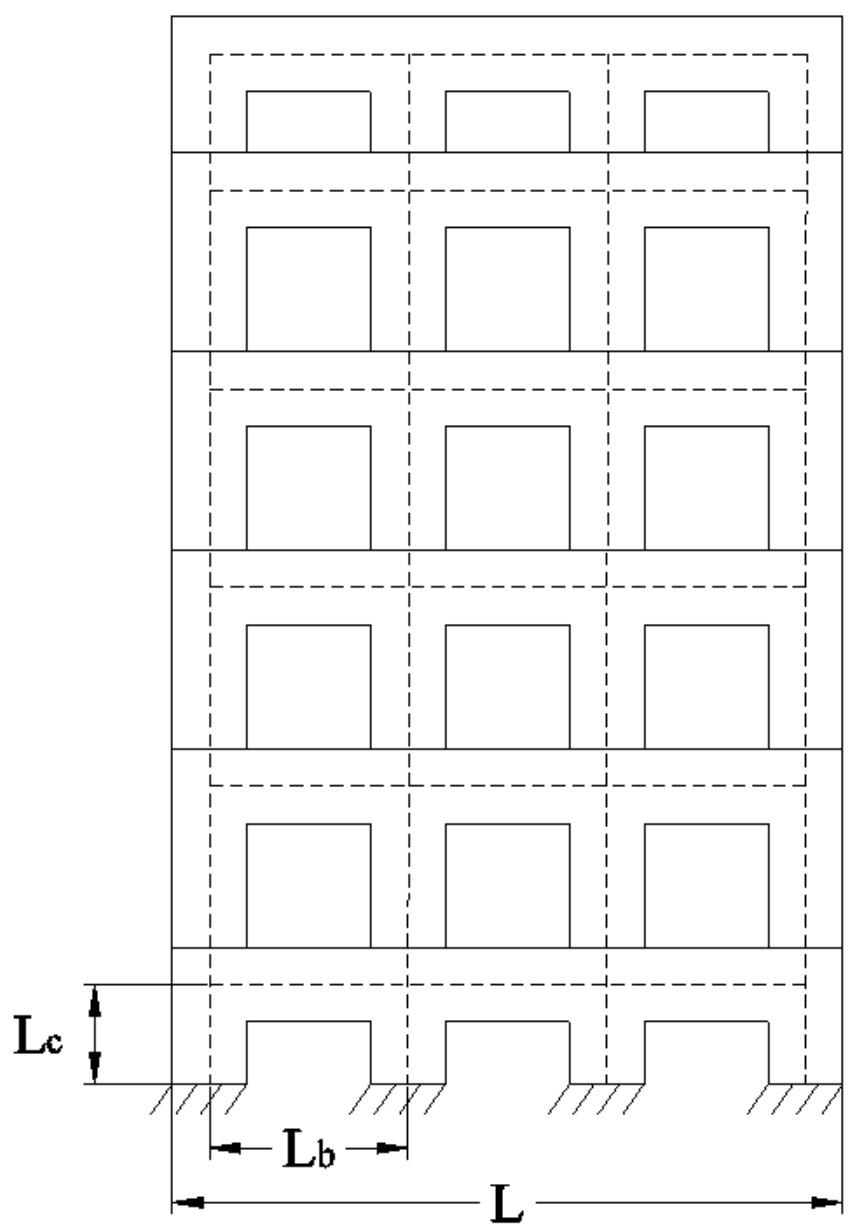

Figure 5.2 Solid shear wall with pier rotation effect.

\subsubsection{Equivalent Shear Media Method (ESM)}

The equivalent shear media method presented by Tena-Colunga and RiveraHerna'ndez (Tena Colunga \& Rivera Hernández, 2015) was used to calculate the lateral intial stiffness of the shear walls with openings. A simplified model was adopted to represent multi-story shear walls with openings by generating a central opening that serves as an equivalent shear media as shown in Figure 5.3. Since this method was 
proposed to calculate the peak lateral displacement of multi-story shear walls, Eq. (5.15) was used to calculate the lateral displacement at each story.

$$
\begin{aligned}
& \alpha=\sqrt{\beta \mu l} \\
& \beta=\frac{12 I_{p} l}{h b^{3}\left(I_{1}+I_{2}\right)} \\
& \mu=1+\frac{\left(A_{1}+A_{1}\right)\left(I_{1}+I_{2}\right)}{A_{1} A_{2} l^{2}} \\
& K_{4}=1-\mu\left(\frac{1}{3}+\frac{\sinh (\alpha H)}{(\alpha H)^{3} \cosh (\alpha H)}-\frac{1}{(\alpha H)^{2}}\right) \\
& \Delta_{\text {shear w/ openings }}=\frac{V(H)^{3} K_{4}}{3 E\left(I_{1}+I_{2}\right)} \\
& \Delta(x)=\frac{V(x)^{3} K_{4}}{3 E\left(I_{1}+I_{2}\right)} \quad 0 \leq x \leq H
\end{aligned}
$$

Where:

$l=$ Length between the centerline of each column surrounding the equivalent shear media

$I_{1}$ and $I_{2}=$ Moment of inertia for the surrounding columns,

$A_{1}$ and $A_{2}=$ Cross-sectional area of the surrounding columns

$I_{p}=$ Moment of inertia for the typical beams connecting the columns

$x=$ Story height from the base of the wall

$H=$ Total height of the wall,

$h=$ Distance between the centerline of beams

$b=$ Equivalent opening width. 

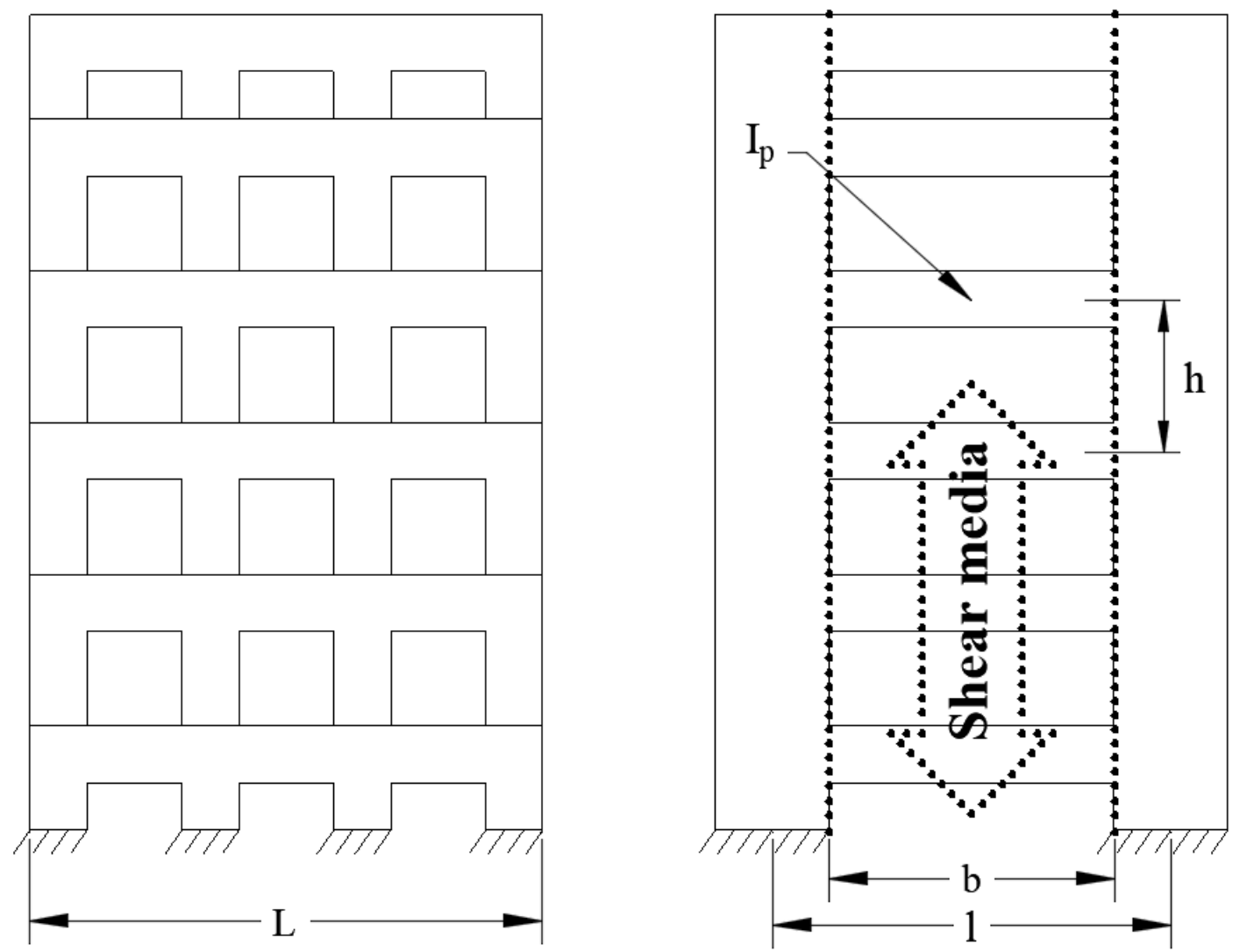

Figure 5.3 Equivalent shear media.

\subsubsection{Simplified Beam-Column Approach (SBCA)}

The beam-column analogy was one of the assumptions that was made to calculate the initial lateral stiffness of the SGICF walls. A simplified approach presented by Yang, $\mathrm{Li}$, and Zhu (Yang, Li, \& Zhu, 2012) to calculate the lateral stiffness of the multi-story frame structures was used. Several assumptions were considered: the elastic behavior for the entire structure, that the joint zones stiffness contribution would be neglected, the negligible axial deformation of the beams, and the assumption that the points of 
inflections would occur at the mid-span for all beams and columns for all stories except the bottom. Thus, the initial lateral displacement of the SGICF walls was calculated by dividing the wall into three regions: top, bottom, and intermediate. As mentioned previously, only the intermediate stories were considered to have points of inflections at the mid-span of the SGICF wall elements, while the offset of points of inflections was considered for the bottom story based on base fixity. As a result, relative displacements were calculated for each story by considering flexural and rotation deformation for both ends as shown in Figure 5.4. The below sections show the required equations to calculate the lateral relative displacements for each story.

\subsubsection{Lateral Relative Displacement of the Intermediate Stories}

$$
\begin{aligned}
& \Delta_{c}=\Delta_{X 1}+\Delta_{X 2}+\Delta_{s 1}+\Delta_{s 2} \\
& \Delta_{c}=2 \frac{V(h / 2)^{3}}{3 E_{c} I_{c}}+\frac{\theta_{s} h}{2}+\frac{\theta_{x} h}{2} \\
& \theta_{s}=\theta_{j}=\frac{M}{K_{j \theta}}=\frac{V\left(h_{j+1}+h_{j}\right)}{2 K_{j \theta}} \\
& \theta_{x}=\theta_{j-1}=\frac{M}{K_{j-1 \theta}}=\frac{V\left(h_{j-1}+h_{j}\right)}{2 K_{j-1 \theta}} ; \quad K_{j-1 \theta}=\sum_{i}^{n_{b}} \frac{6 E_{b} I_{j-1 b}}{L} \\
& K_{j \theta}=\sum_{i}^{n_{b}} \frac{6 E_{b} I_{j b}}{L}
\end{aligned}
$$

Where:

$\Delta_{c}=$ Lateral relative displacement of the ends of the column 
$V=$ Shear force applied on the column

$\Delta_{s 1}$ and $\Delta_{X 1}=$ Lateral displacement of the upper and lower parts of the column, respectively due to the rotation of column ends

$\Delta_{s 2}$ and $\Delta_{X 2}=$ Lateral flexural displacement of the upper and lower ends of the column, respectively

$$
\begin{aligned}
& \theta_{s} \text { and } \theta_{x}=\text { Rotation angles of the top and bottom ends respectively } \\
& h=\text { Column height } \\
& L=\text { Beam length } \\
& n_{b}=\text { Number of beams meeting the column at the end } \\
& j=\text { Story number } \\
& E_{c}=\text { Column modulus of elasticity } \\
& E_{b}=\text { Beam modulus of elasticity } \\
& I_{c}=\text { Column moment of inertia }
\end{aligned}
$$

\subsubsection{Lateral Relative Displacement of the Top Story}

The same equations that were used to calculate the relative lateral displacement of the intermediate story was used for the top story except for the term $h_{j+1}$ that was eliminated.

\subsubsection{Lateral Relative Displacement of the Bottom Story}

The lateral displacement of the bottom story was calculated differently than that of the intermediate stories. The inflection points were shifted toward the end because the 
columns were fixed at the base where moment resisting stiffness was smaller. Therefore, and for the standard frame, $y_{1}$ and $y_{2}$ calculated as shown below:

$$
\begin{gathered}
y_{1}=\frac{\frac{3 E_{b} I_{b}}{L}+\frac{E_{c} I_{c}}{h_{1}} \frac{\left(h_{1}+h_{2}\right)}{2 h_{1}}}{\frac{6 E_{b} I_{b}}{L}} h_{1} \\
y_{2}=\frac{\frac{3 E_{b} I_{b}}{L}-\frac{E_{c} I_{c}}{h_{1}} \frac{\left(h_{1}+h_{2}\right)}{2 h_{1}}}{\frac{6 E_{b} I_{b}}{L}} h_{1}
\end{gathered}
$$

Lateral relative displacement of the bottom story was calculated as:

$$
\Delta_{\mathrm{c}}=\Delta_{X 2}+\Delta_{s 1}+\Delta_{s 2}
$$

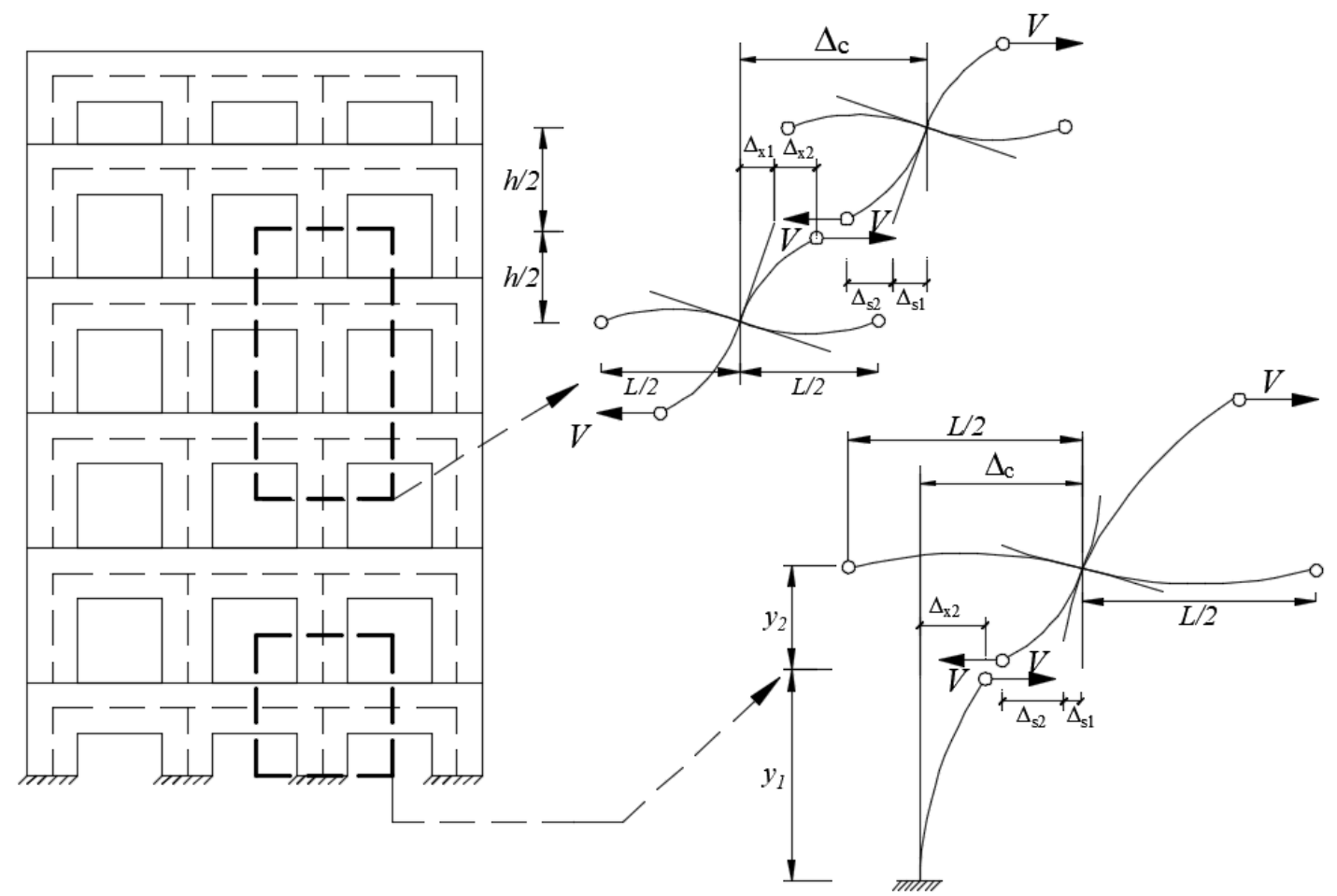

Figure 5.4 Simplified beam column approach. 


$$
\Delta_{\mathrm{c}}=\frac{V\left(y_{1}\right)^{3}}{3 E_{c} I_{c}}+\theta_{s} y_{2}+\frac{V\left(y_{2}\right)^{3}}{3 E_{c} I_{c}}
$$

\subsubsection{Initial Stiffness Results}

Table 5-1 shows the analytical initial stiffness of the SGICF walls obtained from each analytical approach with a comparison to the experimental intial stiffness obtained from the free vibration tests. For instance, the SOPR method was found to be overestimating the initial stiffness of all SGICF walls. This overestimation was reduced by more than half by considering the rotation of the piers as was described in the SWPR approach. Although, considering the rotation of the piers in the SWPR method reduced the initial stiffness estimation by a half, the SWPR method still overestimated the initial stiffness. On average, the initial stiffness values obtained from the SWPR were 4.1 times greater than the values obtained from the experimental tests.

The initial analytical stiffness calculated by using the SBCA method showed close values to that obtained from the SWPR approach for W-RC and W-FB walls. Meanwhile, the initial stiffness calculated for G-RC and G-FB by the SWPR method was higher than SBCA method by $160 \%$. Again, the SBCA method did not provide a suitable estimation for the initial stiffness of all SGICF walls. On average, the initial stiffness values obtained from the SBCA method were 3.2 times greater than the values obtained from the experimental tests.

The ESM method revealed an estimation for the initial stiffness that was $80 \%$ higher than the experimental values for W-RC and G-FB walls. Whereas it was 100\% 
higher for W-FB wall and 70\% for G-RC. Therefore, and among all the presented analytical methods, the ESM method provided the closest estimations for the initial stiffness of the SGICF walls with an average of 1.83 times the values of the experimental tests.

Table 5-1 Comparison of analytical initial stiffness with experimental results.

\begin{tabular}{|c|c|c|c|c|c|c|c|c|c|}
\hline \multirow{2}{*}{$\begin{array}{c}\text { Wall } \\
\text { Designation }\end{array}$} & \multicolumn{4}{|c|}{ Analytical Initial Stiffness } & Exp.intial & & & & \\
\cline { 2 - 9 } Stiffness & $\frac{50 P R}{\text { Exp. }}$ & $\frac{\text { SWPR }}{\text { Exp. }}$ & $\frac{\text { ESM }}{\text { Exp. }}$ & $\frac{\text { SBCA }}{\text { Exp. }}$ \\
\hline W-RC & 468 & 206 & 101 & 203 & 55.8 & 8.4 & 3.7 & 1.8 & 3.6 \\
\hline W-FB & 441 & 194 & 95 & 191 & 47.4 & 9.3 & 4.1 & 2.0 & 4.0 \\
\hline G-RC & 347 & 143 & 58 & 88 & 34.7 & 10.0 & 4.1 & 1.7 & 2.5 \\
\hline G-FB & 324 & 133 & 54 & 82 & 29.4 & 11.0 & 4.5 & 1.8 & 2.8 \\
\hline
\end{tabular}

\subsection{Strength Capacity of SGICF Walls}

To date, several methods were developed and introduced to predict the shear capacity of the shear walls. Many assumptions and limitations were considered to calculate a SGICF wall's theoretical shear capacity. In this section, four methods were used to calculate the shear capacity of SGICF walls. 


\subsubsection{Summation of Individual Vertical Core Plastic Moment Capacity $\left(\sum \mathrm{V}_{\mathrm{cm}}\right)$}

The plastic moment capacity of each vertical core was calculated to estimate the shear capacity of the SGICF by using Eq. (5.21). Therefore, many assumptions were made such as plane sections remain plane after bending; equivalent rectangular stress block for compression zone expressed for stress distribution; maximum strain in the extreme fiber of concrete in compression was 0.003 ; concrete tensile strength was negligible; and the steel reinforcement material was an elasto-plastic (Aydemir, Zorbozan, \& Alacali, 2009). The shear capacity of the SGICF wall was the result of the summation of its cores strength. Finally, the effect of ICF forms was neglected.

$$
\sum \mathrm{V}_{\mathrm{CM}}=\frac{2 * \mathrm{M}_{\mathrm{pc}}}{\mathrm{h}} * \mathrm{n}
$$

Where:

$$
\begin{aligned}
& \mathrm{M}_{\mathrm{pc}}=\text { Cross-sectional plastic moment } \\
& \mathrm{h}=\text { Distance between the horizontal cores } \\
& \mathrm{n}=\text { Vertical cores number }
\end{aligned}
$$

\subsubsection{Consideration of Reinforced Vertical Concrete Cores Only $\left(\mathrm{V}_{\mathrm{C}}\right)$}

The in-plane shear resistance of the SGICF walls was estimated by using the ACI equation (ACI 318-14, 2014) for the minimum shear strength of the concrete. Many assumptions were made to utilize this equation. The first assumption was to ignore the axial loads, both self-weight and any additional gravity loading, to which the wall may be 
subjected. The second assumption was to consider the action of the vertical cores of the SGICF walls as a single wall. For clarification, an $8 \mathrm{ft}$. wall had six cores, and each core was 6 in. long, thus the equivalent wall length would be 36 in. (six times 6 in.). This assumption ignored the contribution of the horizontal cores, as well as any contributions from the steel and wall forms. The total shear capacity of each SGICF wall was calculated using Eq. (5.22).

$$
\mathrm{V}_{\mathrm{C}}=2 \lambda \sqrt{\mathrm{f}_{\mathrm{c}}^{\prime}} b_{w} \mathrm{~d}
$$

Where:

$\lambda=$ Shear strength reduction factor

$f^{\prime} c=$ Concrete compressive strength (psi)

$b_{w}=$ Thickness of SGICF vertical core (in)

$d=0.8$ the equivalent wall length

\subsubsection{Shear Capacity Due to the Plastic Moment of the Whole Wall ( $\left.\mathrm{V}_{\mathrm{MW}}\right)$}

Differently from considering the plastic moment of each vertical core, this method was used to estimate the SGICF wall's shear capacity by considering the plastic moment of the whole wall. In this method, the assumption was made to neglect the effect of compression force due to steel bars at the compression zone, if any, to the concrete compression capacity. The calculation of plastic moment was conducted for each wall and the total base shear capacity became the product of dividing wall's plastic moment by the wall's height. 


\subsubsection{Shear Capacity by using ICC ESR 1770 Equations $\left(\mathrm{V}_{\mathrm{C}}(\mathrm{ICC})\right)$}

The International Code Council Evaluation Service (ICC-ES) released a report (ICC Evaluation Service, 2012) describing the required criteria when using EPS ICF systems to conform to the International Building Code (IBC) and the Uniform Building Code (UBC). The report provided Eq. (5.23) to calculate the nominal shear strength of members that are subjected to shear and flexure.

$$
\begin{aligned}
& \mathrm{V}_{\mathrm{C}}(\mathrm{ICC})=2 \varphi \psi b_{e q} \mathrm{~d} \sqrt{\mathrm{f}_{\mathrm{c}}^{\prime}} \\
& \text { Where: } \\
& b_{e q}=\text { Width of an equivalent rectangular section (4.5 in) } \\
& f^{\prime} c=\text { Concrete compressive strength (psi) } \\
& d=0.8 l \\
& l=\text { Length of the wall (in) } \\
& \psi=\text { Grid factor of } 0.85 \\
& \varphi=\text { Strength reduction factor based on applicable codes }
\end{aligned}
$$

\subsubsection{Shear Strength Results}

Table 5-2 shows the analytical lateral strength results obtained from each method for all of the SGICF walls with a comparison to the experimental peak lateral loads. The results obtained from $\sum \mathrm{V}_{\mathrm{cm}}$ method showed very good agreement with the experimental results. On average, the theoretical values obtained by $\sum \mathrm{V}_{\mathrm{cm}}$ method were 1.03 times the 
experimental results. This precise estimation of the peak lateral strength makes $\sum \mathrm{V}_{\mathrm{cm}}$ method as one of the successful approaches for estimating the peak lateral strength of the SGICF walls.

The results obtained from the ACI equation for the minimum shear strength of the concrete also provided a very good estimation for the peak lateral strength of the SGICF walls as well. As shown in Table 5-2, this method managed to estimate the peak lateral strength with an average of 1.03 times the experimental results. Also, it was shown that the experimental values were 1.05 times the values obtained from the $V_{c}$ method for both G-RC and G-FB walls. Further, the peak lateral strength obtained for W-FB wall is in question due to the voids found in its cores, and makes the comparison with the analytical approaches has some uncertainties.

The analytical results of the peak lateral strength calculated by $\mathrm{V}_{\mathrm{mw}}$ method for W-RC and W-FB walls showed promising estimation, and on average the experimental values were 1.05 times the values obtained from the $\mathrm{V}_{\mathrm{mw}}$ method. This estimation was not true for the analytical values of the new SGICF walls, since $V_{m w}$ overestimated the peak lateral strength of these walls by an average of 1.7 times the experimental values.

The equation that was proposed by the international code council report to estimate the in-plane lateral strength of the EPS ICF walls proved to overestimating the peak lateral strength. On average, the theoretical values obtained by $V_{c}$ (ICC) method were 1.46 times the experimental results. In conclusion, the summation of individual vertical core plastic moment and the ACI equation methods succeeded in providing a very close estimation of the in-plane lateral strength of the SGICF walls. 
Table 5-2 Comparison of theoretical peak lateral strength and peak experimental loads.

\begin{tabular}{|c|c|c|c|c|c|c|c|c|c|}
\hline \multirow{2}{*}{$\begin{array}{c}\text { Wall } \\
\text { Designation }\end{array}$} & \multicolumn{4}{|c|}{$\begin{array}{l}\text { Analytical Lateral Strength } \\
\text { (kip) }\end{array}$} & \multirow{2}{*}{$\begin{array}{l}\text { Exp. } \\
\text { (kip) }\end{array}$} & \multirow{2}{*}{$\frac{\Sigma \mathrm{Vcm}}{\text { Exp. }}$} & \multirow{2}{*}{$\frac{\mathrm{V}_{\mathrm{c}}}{\operatorname{Exp}}$} & \multirow{2}{*}{$\frac{V_{\text {mw }}}{\text { Exp. }}$} & \multirow{2}{*}{$\frac{\mathrm{V}_{\mathrm{c}}(\mathrm{ICC})}{\operatorname{Exp}}$} \\
\hline & $\Sigma \mathrm{Vcm}$ & $V_{c}$ & Vmw & $\begin{array}{c}\mathrm{V}_{\mathrm{c}} \\
(\mathrm{ICC})\end{array}$ & & & & & \\
\hline W-RC & 16.0 & 18.2 & 15.4 & 23.1 & 18.2 & 0.88 & 1.0 & 0.85 & 1.27 \\
\hline W-FB & 15.8 & 17.1 & 15.34 & 21.8 & 14.0 & 1.13 & 1.22 & 1.1 & 1.55 \\
\hline G-RC & 14.44 & 13.3 & 22.94 & 21.2 & 14.0 & 1.0 & 0.95 & 1.64 & 1.51 \\
\hline G-FB & 14.25 & 12.4 & 22.85 & 19.8 & 13.0 & 1.1 & 0.95 & 1.76 & 1.52 \\
\hline
\end{tabular}

\subsection{Summary}

Variety of analytical approaches that were presented by previous researchers to estimate the initial stiffness and the maximum lateral strength of the shear walls with openings were adopted in this chapter. Many assumptions were made to assure the applicability of each method on the SGICF walls. A comparison was made between the analytical and experimental results to validate the suitability of these approaches. The equivalent shear media method was the only approach that provided a close estimation of the initial stiffness for all SGICF walls. The results obtained from using the ACI equation for the minimum shear strength of the concrete were accurate in estimating the lateral strength of the three walls. The W-FB wall was the only wall that this method overestimated its lateral strength by $22 \%$, and this can be justified by the voids found in the W-FB wall, which influenced its lateral strength. The $\Sigma \mathrm{Vcm}$ method was the second method that managed to provide a good estimation for the lateral strength of the SGICF walls with an average overestimation ratio of $103 \%$ for all walls. 


\section{CHAPTER 6: Numerical Analysis}

\subsection{General}

A nonlinear analysis was performed on each SGICF wall using the finite element program SAP2000 v19.2.1 Ultimate that was developed by Computer and Structures Inc. (Computers and Structures Inc., (CSI), 2017). The goal was to obtain the lateral capacity curves, failure loads, and displacements of the SGICF walls. This section includes the description of the numerical model, material, loading, hinges types, analysis results, comparison with the experimental results, and the influence of EPS blocks on the lateral strength. Since the experimental tests were performed to study the in-plane behavior of the SGICF walls, a 2D model was created and analyzed.

\subsection{Numerical Model}

\subsubsection{Finite Element Model Description and Materials}

The SGICF walls were modeled as a grid of columns and beams with fixed supports at the bottom. Each member was assigned to a frame element, which was modeled as a straight line joining two points, and used a three-dimensional beam-column formulation including biaxial bending, torsion, axial deformation, and biaxial shear deformations (Cook, Malkus, Plesha, \& Witt, 2001). A section designer feature was used to define the SGICF wall elements' cross-sectional dimensions and reinforcement details. The EPS blocks were modeled as masonry infills in-plane as was described in FEMA 356 report (American Society of Civil Engineering (ASCE), 2000). Figure 6.1 shows the 
concentric compression strut analogy that was used to model the EPS blocks. The thickness of the compression struts was assigned to the thickness of the wall, which was assigned to the diameter of the vertical or the horizontal cores. The dimensions of the compression struts were found according to the equation that was provided by the FEMA 356 report as follows:

$$
\mathrm{a}=0.175\left(\lambda_{1} \mathrm{~h}_{\mathrm{col}}\right)^{-0.4} \mathrm{r}_{\mathrm{inf}}
$$

Where:

$\lambda_{1}=\left[\frac{E_{\mathrm{me}} \mathrm{t}_{\text {inf }} \sin 2 \theta}{4 \mathrm{E}_{\mathrm{fe}} \mathrm{I}_{\mathrm{col}} \mathrm{h}_{\mathrm{inf}}}\right]^{1 / 4}$

and,

$\mathrm{h}_{\mathrm{col}}=$ Column height between centerlines of beams, in.

$\mathrm{h}_{\text {inf }}=$ Height of infill panel, in.

$\mathrm{E}_{\mathrm{fe}}=$ Expected modulus of elasticity of frame material, ksi.

$\mathrm{E}_{\mathrm{me}}=$ Expected modulus of elasticity of infill material, ksi.

$\mathrm{I}_{\mathrm{col}}=$ Moment of inertia of column, $\mathrm{in}^{4}$.

$\mathrm{L}_{\mathrm{inf}}=$ Length of infill panel, in.

$r_{\text {inf }}=$ Diagonal length of infill panel, in.

$\mathrm{t}_{\mathrm{inf}}=$ Thickness of infill panel and equivalent strut, in.

$\theta=$ Angle whose tangent is the infill height-to-length aspect ratio, radians .

$\lambda_{1}=$ Coefficient used to determine the equivalent width of infill strut. 


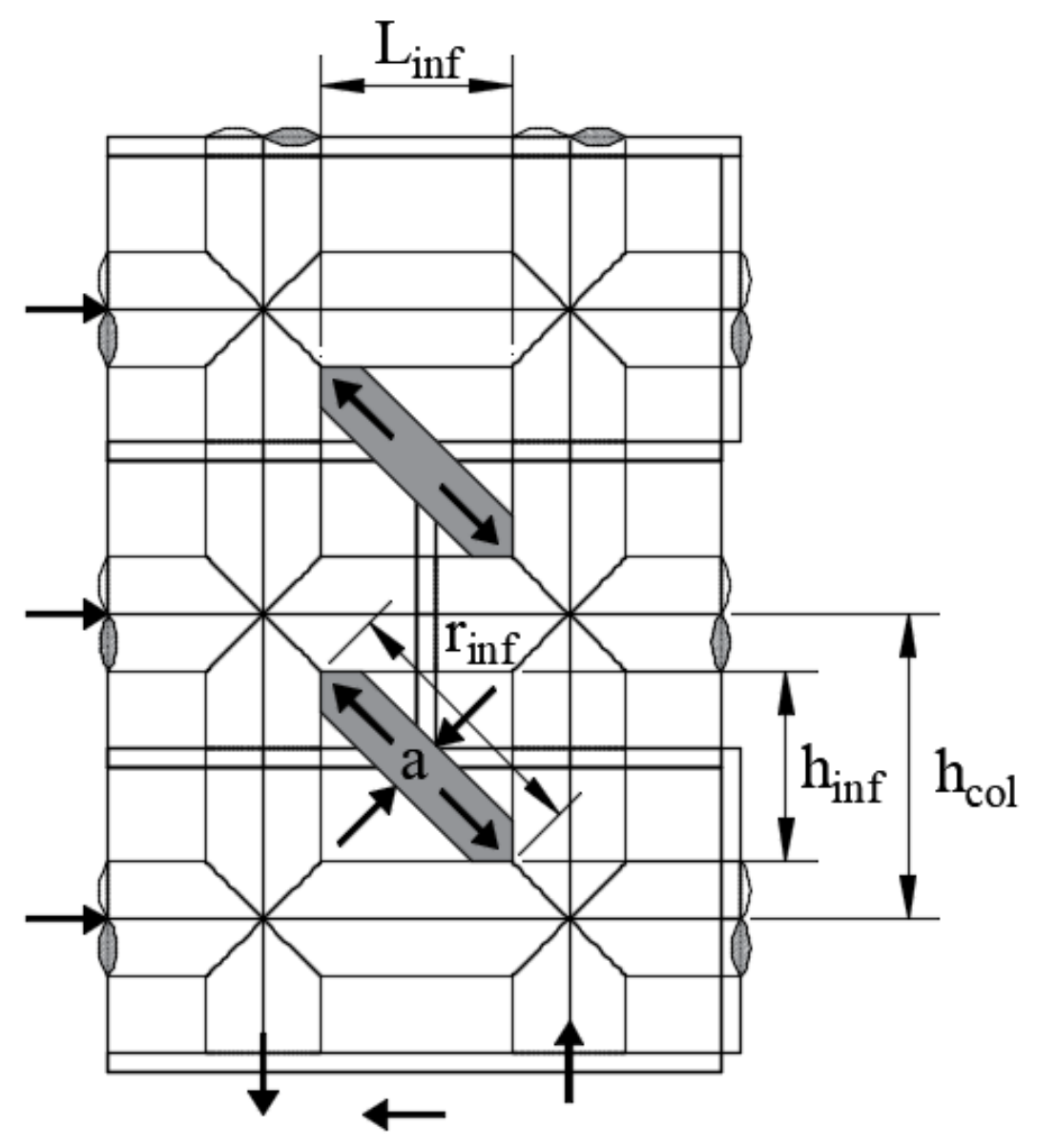

Figure 6.1 Compression concentric struts analogy.

Werner (Werner, 2010) conducted compressive strength tests on different types of EPS blocks, and provided the stress-strain relationships for each sample as shown in Figure 6.2. The modulus of elasticity of the EPS blocks was found from the best fit of the linear elastic part of the stress-strain curve and was set to 4800 psi. The same value of the Poisson's ratio of the EPS material that was found experimentally as 0.43 by Yland (Yland, 2000), and was used in the numerical model. Figure 6.3 shows the stress-strain 
curve of the EPS material that was the best fit representation of the stress-strain curve of the EPS material that was provided by Werner.

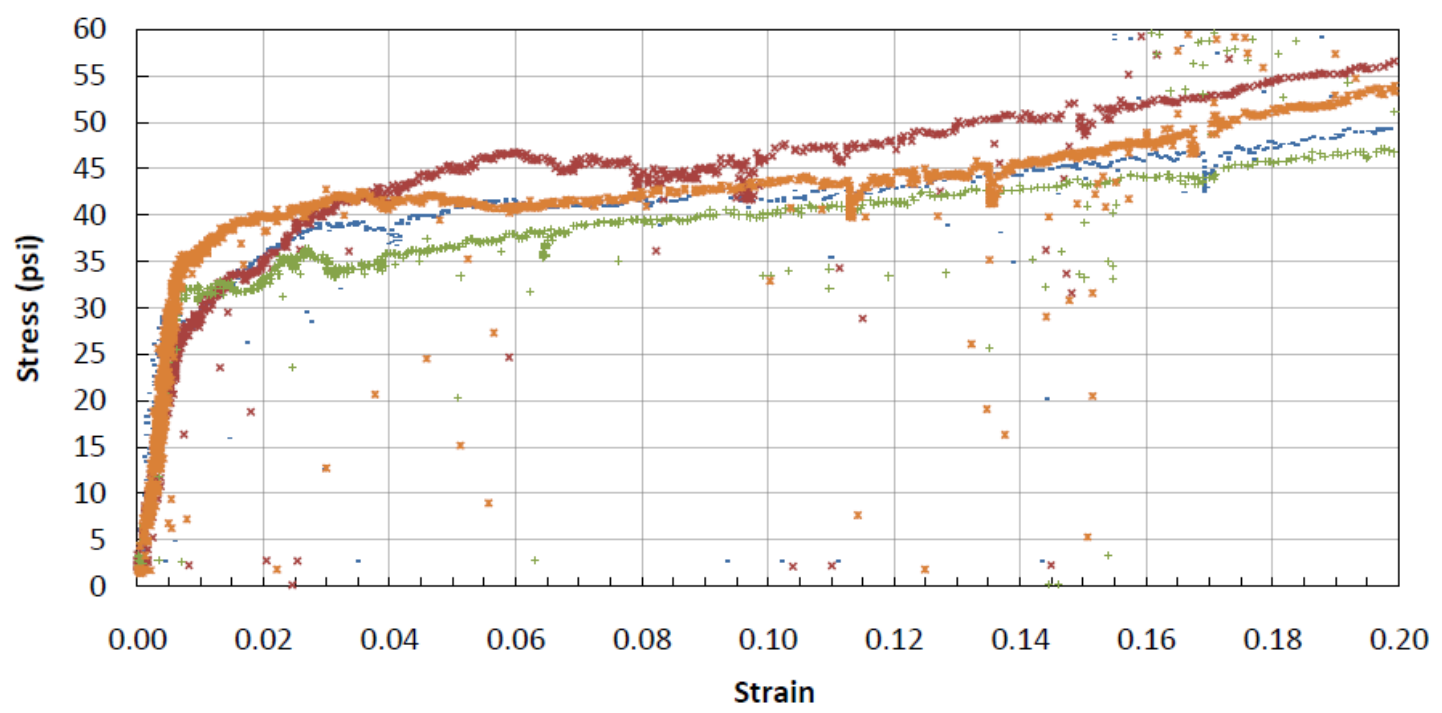

Figure 6.2 Compression tests of EPS material (Werner, 2010).

Figure 6.4 and Figure 6.5 show the nonlinear stress-strain curves for the concrete and rebar steel used in the numerical model. For each tested wall, the compressive strength that resulted from the compression tests of the concrete cylinders on the day of the test was used in defining the reinforced concrete properties. The stress-strain curve shown in Figure 6.4 is an example of the concrete stress-strain curve, which was defined based on the compressive strength test results of the concrete cylinders of the W-RC wall. The yield and ultimate stresses of the rebar were obtained from the material test as shown in Figure 3.12. Since the rebar steel that was used in all of the SGICF walls came from 
the same steel batch, the stress-strain curve shown in Figure 6.5 was used in modeling the rebar for all the SGICF walls.

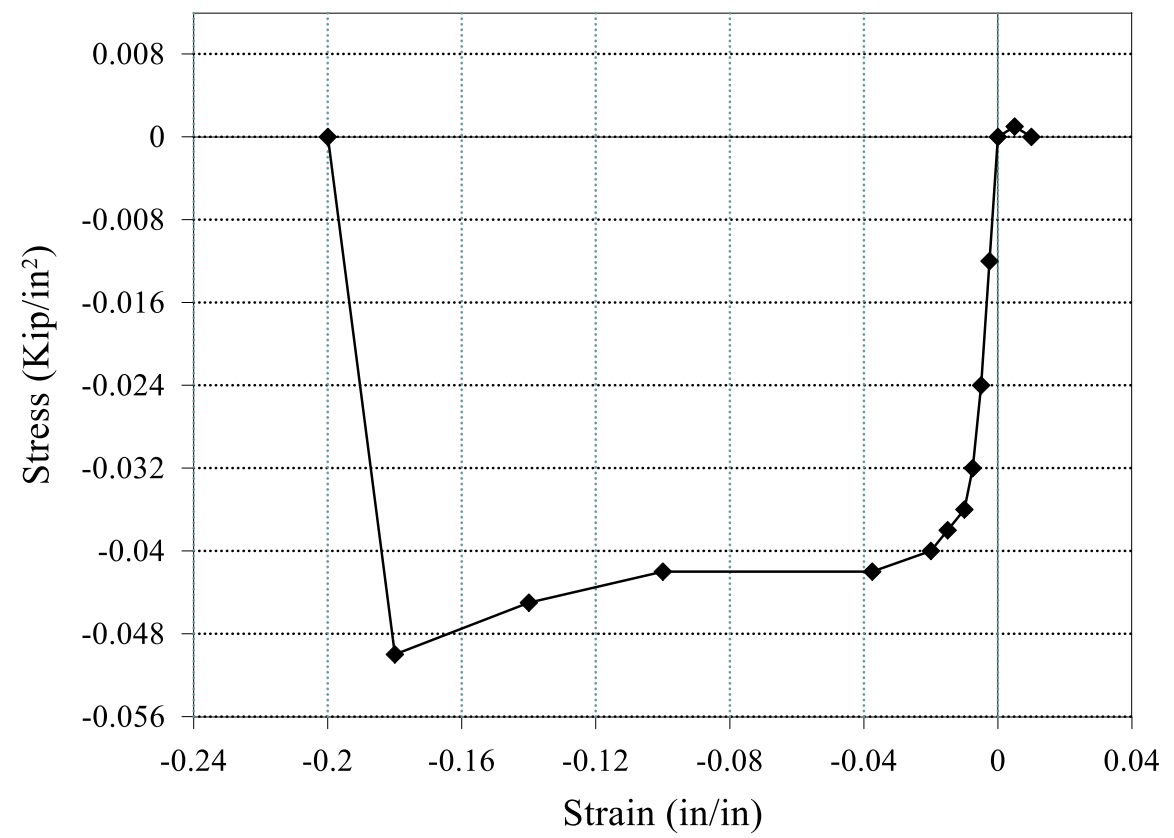

Figure 6.3 Nonlinear stress-strain curve for EPS block used in the numerical model. 


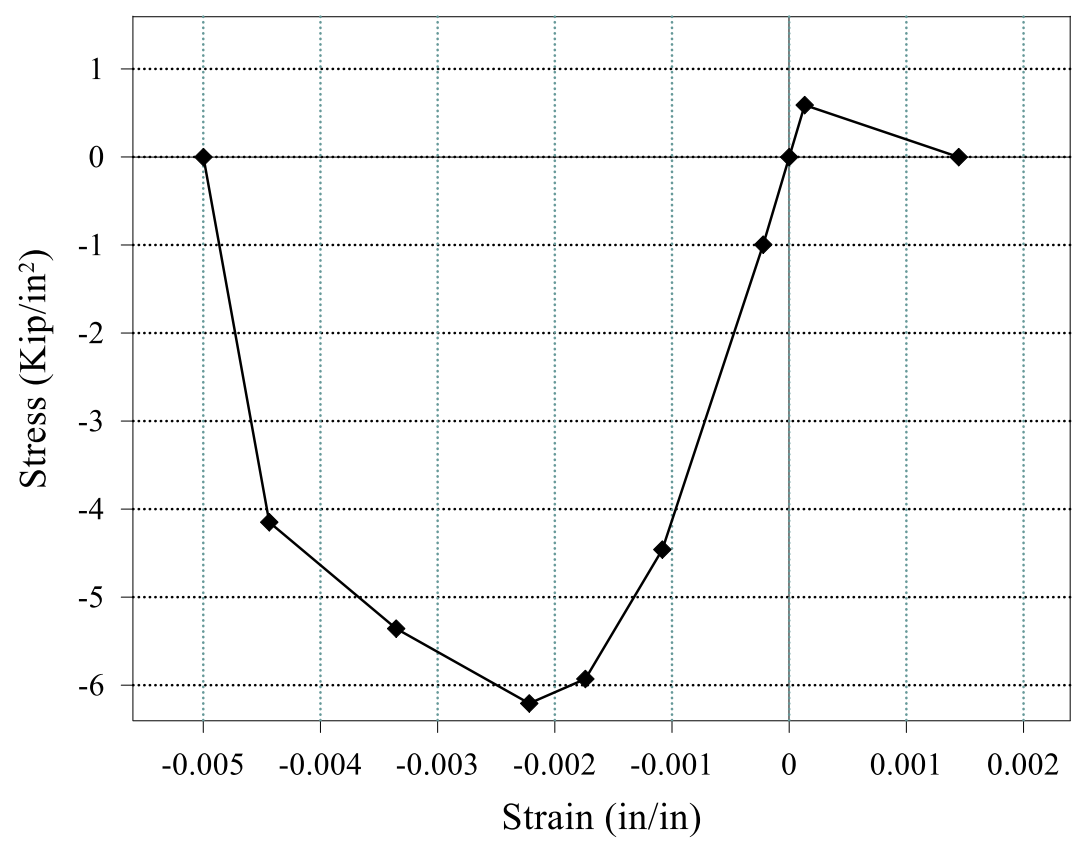

Figure 6.4 Nonlinear stress-strain curve for concrete (W-RC wall) used in the numerical model.

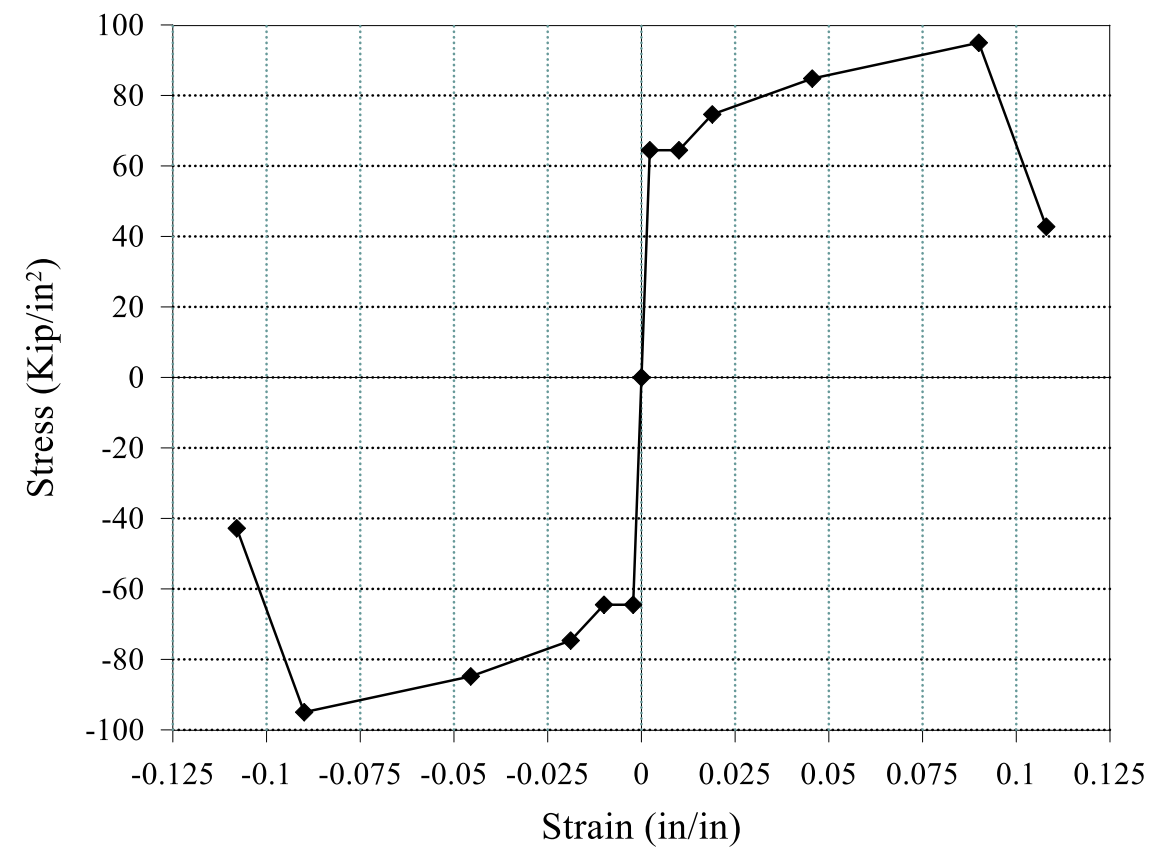

Figure 6.5 Nonlinear stress-strain curve for steel rebar used in the numerical model 


\subsubsection{Pushover Analysis}

Pushover analysis is a quasi-static nonlinear analysis where the structural buildings or elements will be subjected to an incrementally increased lateral displacement in order to find the failure modes or the weakest links (Habibullah \& Pyle, 1998). In SAP2000, the material nonlinearity in frames is handled by the creation of concentrated plastic hinges (Computer \& Structures INC., 2011) . The location of these hinges has to be assigned manually by the user. For the frame elements, SAP2000 permits the user to choose between two different types of plastic hinges, automatic hinges, and user-defined hinges. In this study, Fiber P-M2-M3 hinges were assigned to all the structural members. It was proven that models with fiber-based hinges (distributed plasticity models) provided more accurate results when compared to models that use plasticity-based hinges, especially when high axial deformation is influential to the structural behavior (Computer \& Structures INC, 2016). The inelastic response was obtained by analyzing the multiple discretized cross-sections as shown in Figure 6.6.

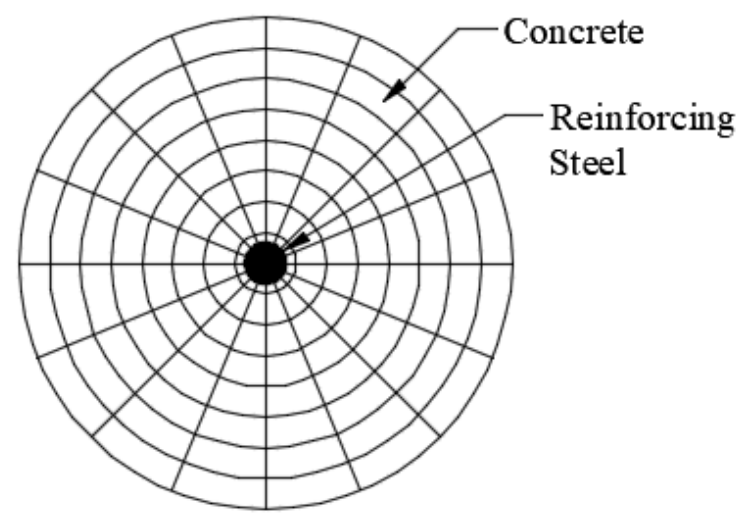

Figure 6.6 Discretized fiber section. 
The cross-sections of the vertical and horizontal cores were divided into two types of fibers for different kinds of materials. To illustrate, one rebar fiber and 240 unconfined concrete fibers were assigned to the circular cross-section shown in Figure 6.6. Selecting a higher number of fibers requires high computer storage and execution time; therefore a judgment in selecting fibers is necessary to get a balance between accuracy and computational efficiency. The fiber hinges were assigned at the ends of each member except for the EPS struts where the plastic hinges were assigned at the mid-span.

\subsubsection{Numerical Analysis Results}

The pushover analysis was performed for all the SGICF walls, and compared with the data from the shake table experiments. The results obtained through the pushover analysis using the section designer fiber hinges models in SAP2000 compared to the experimental tests are shown in Figure 6.7.

From the results obtained for W-RC and W-FB walls, it was possible to observe that, in general, the pushover curves were pretty close to those obtained from the experimental program. The peak lateral loads were close to the experimental results with a $17 \%$ difference in underestimation for $\mathrm{W}-\mathrm{RC}$ and $8 \%$ difference in overestimation for W-FB. Also, the initial stiffness of W-RC was $9 \%$ difference less and $68 \%$ more for WFB. Table 6-1 shows the differences in the initial stiffness and peak lateral loads obtained from both the numerical models and the experimental tests. Pushover analysis results showed an underestimation of the peak lateral loads for all walls, except for the W-FB 
wall. Voids found in some of the W-FB cores contributed to the lower lateral strength measured from the experimental tests. The pushover curves for the G-RC and G-FB walls succeeded in estimating the peak lateral loads as shown in Figure 6.7. The initial stiffness of $\mathrm{G}-\mathrm{RC}$ was higher than the experimental results by $7.5 \%$ and lower by $34 \%$ for G-FB as it shown in Table 6-1.

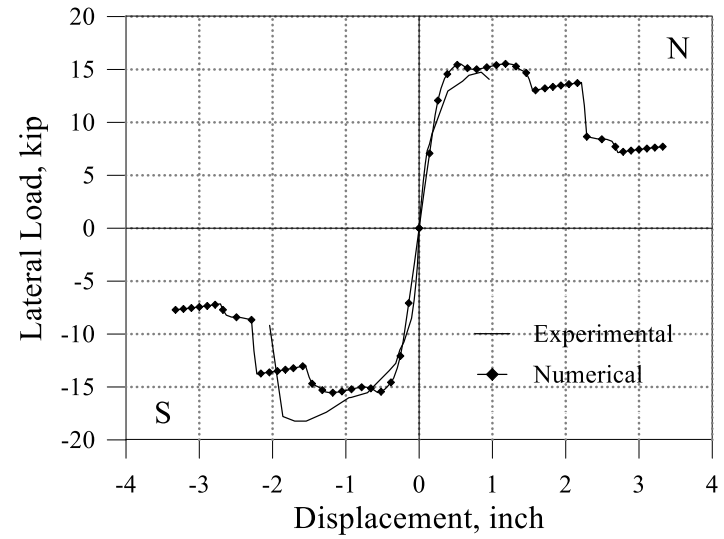

(a) W-RC

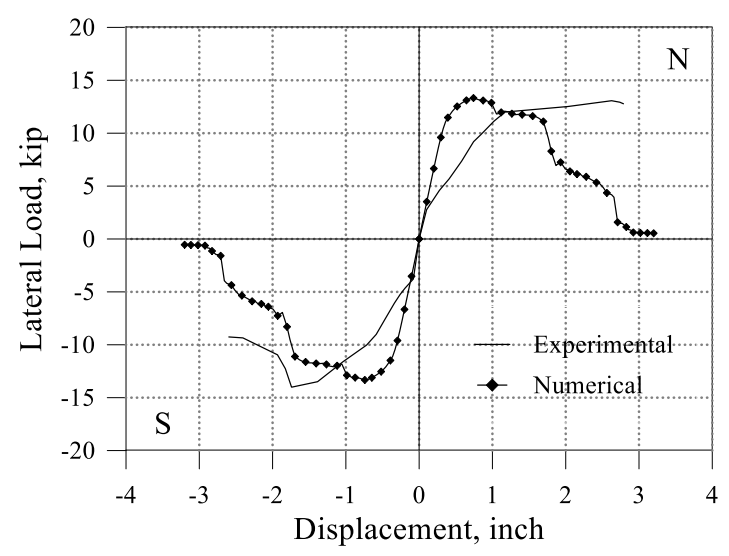

(c) G-RC

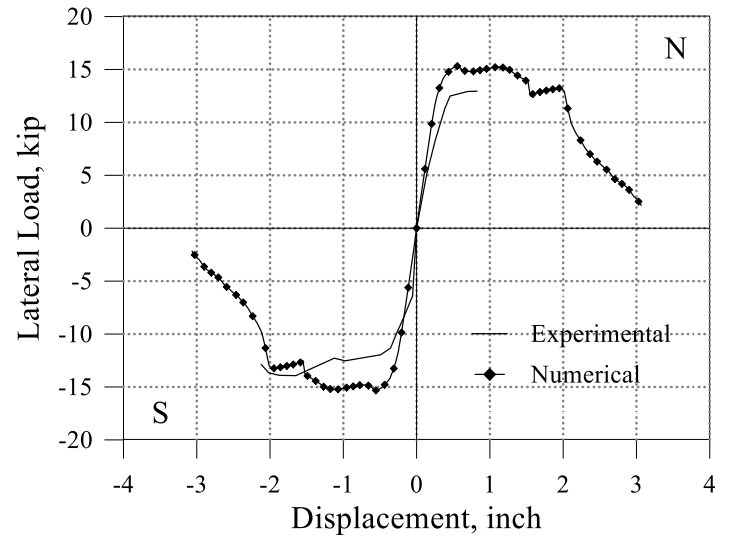

(b) W-FB

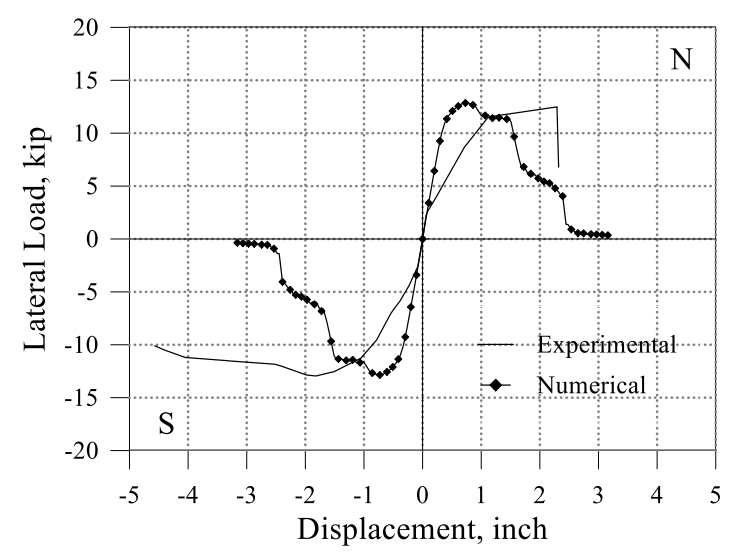

(d) G-FB

Figure 6.7 Comparison of experimental and numerical load-displacement curves. 
SAP2000 provided information about the behavior of the discretized layers of each section within the fiber plastic hinges of each wall during the pushover analysis steps. Since each steel rebar was assigned to one layer, an investigation was made to find when each section reached the yielding stage and how that influenced the overall behavior of each SGICF wall. The distribution of the reinforcement yielding in the fiber hinges vs. the pushover steps of the W-RC wall is shown in Figure 6.8. It was shown that the yielding of the vertical rebar played a significant role in the nonlinear behavior of the W-RC wall. Figure 6.9 shows the distribution of the yielding stage of each of the fiber plastic hinges during the pushover steps for the W-FB wall, which showed similar behavior to the W-RC wall.

The yielding of the horizontal cores reinforcement dominated the collapse mechanism of both G-RC and G-FB walls as it is shown in Figure 6.10 and Figure 6.11. The influence of using two rebars in each vertical core was noticeable during the early steps. The effect of vertical cores reinforcement yield caused the deterioration of the overall strength that led to strength reduction and the collapse of the walls.

Table 6-1 Initial stiffness and peak load comparisons between numerical models and experimental results.

\begin{tabular}{|c|c|c|c|c|c|c|}
\hline \multirow{2}{*}{$\begin{array}{c}\text { Wall } \\
\text { Designation }\end{array}$} & \multicolumn{2}{|c|}{$\begin{array}{c}\text { Peak Lateral Load } \\
\text { (kip) }\end{array}$} & \multirow{2}{*}{$\begin{array}{c}\text { Difference } \\
(\%)\end{array}$} & \multicolumn{2}{|c|}{$\begin{array}{c}\text { Initial stiffness } \\
\text { (kip/in) }\end{array}$} & \multirow{2}{*}{$\begin{array}{c}\text { Difference } \\
\text { nnnnnnn}\end{array}$} \\
\cline { 2 - 3 } & Exp. & Numerical & & Exp. & Numerical & \\
\hline W-RC & 18.2 & 15.54 & -17 & 55.8 & 51.2 & -9 \\
\hline W-FB & 14.0 & 15.3 & +9 & 29.4 & 49.5 & +68 \\
\hline G-RC & 14.0 & 13.3 & -5 & 34.7 & 37.3 & +7.5 \\
\hline G-FB & 13.0 & 12.9 & -1 & 47.4 & 35.3 & -34 \\
\hline
\end{tabular}



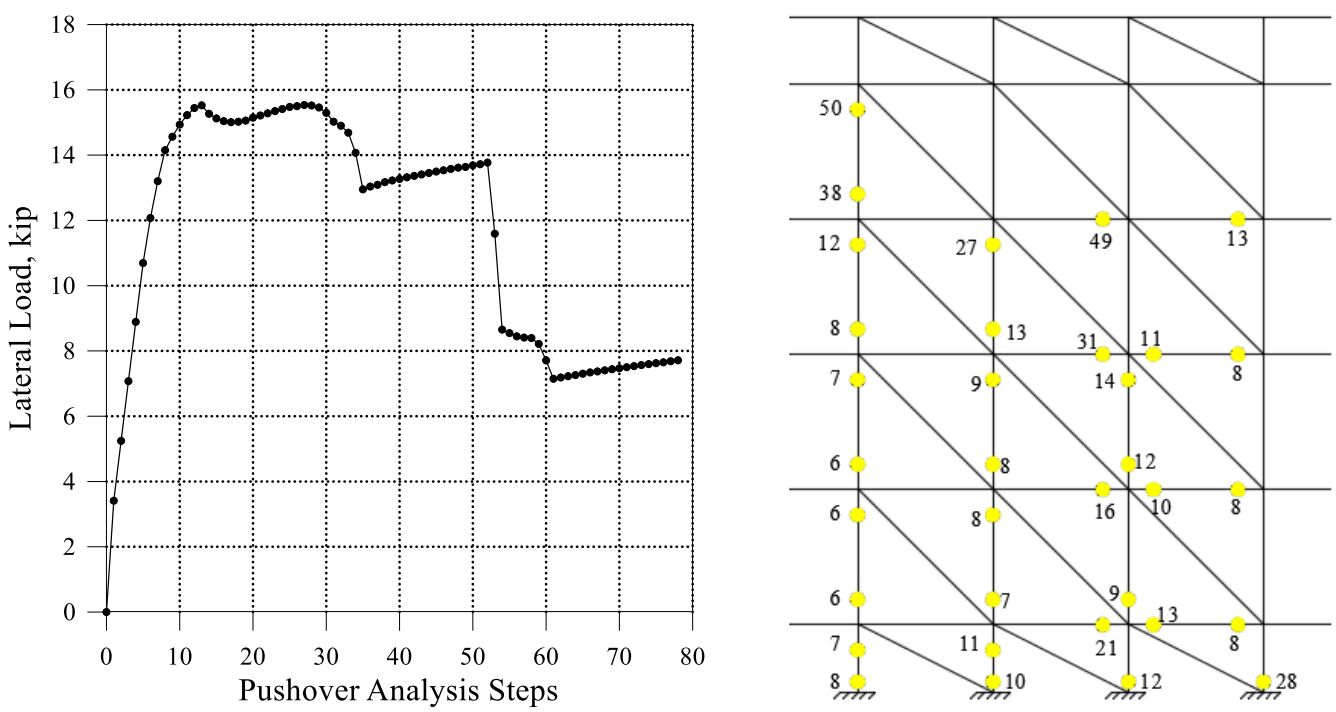

Figure 6.8 Fiber plastic hinges reinforcement yield progress mechanism for W-RC wall.
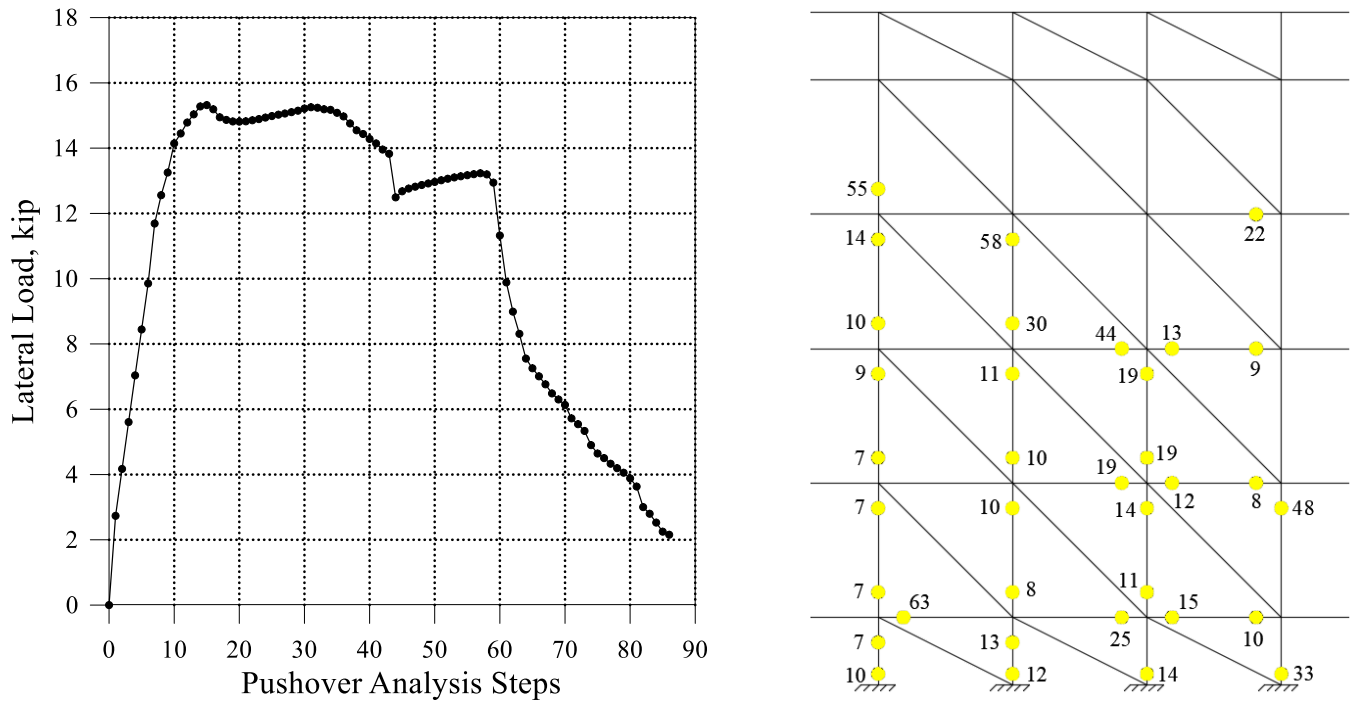

Figure 6.9 Fiber plastic hinges reinforcement yield progress mechanism for W-FB wall. 

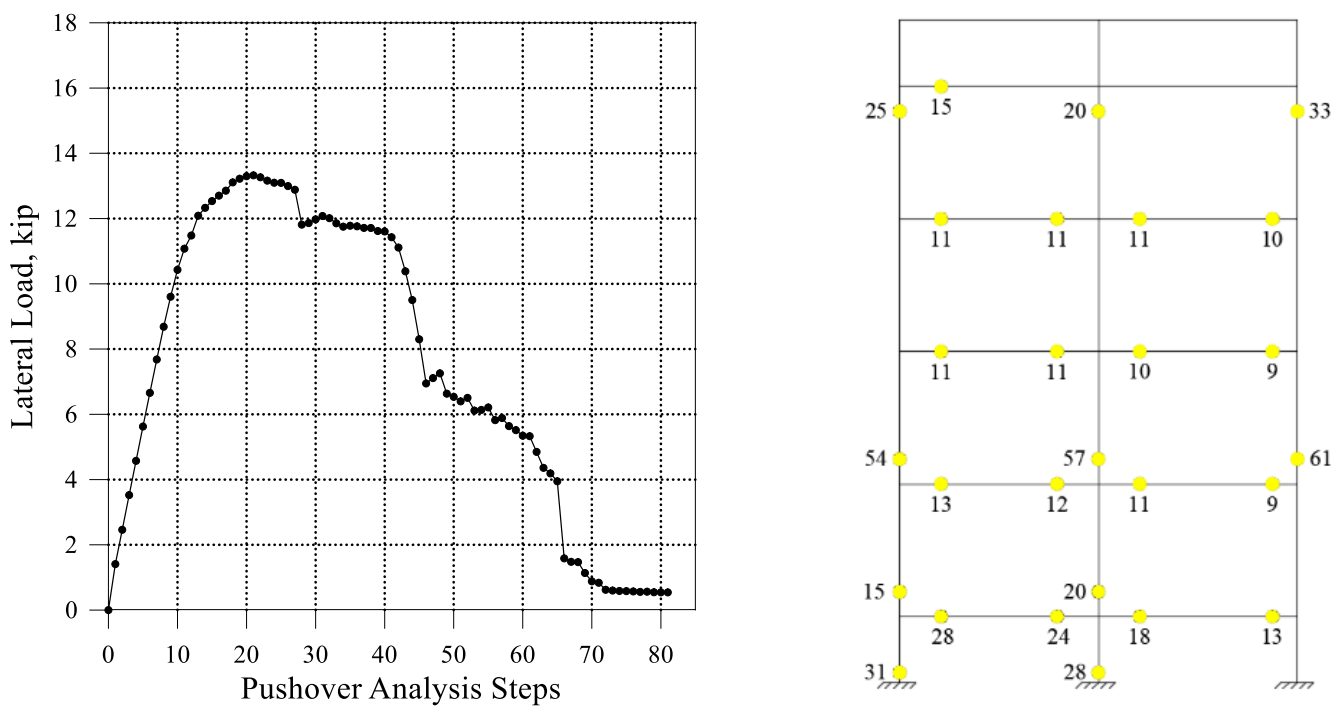

Figure 6.10 Fiber plastic hinges reinforcement yield progress mechanism for G-RC wall.
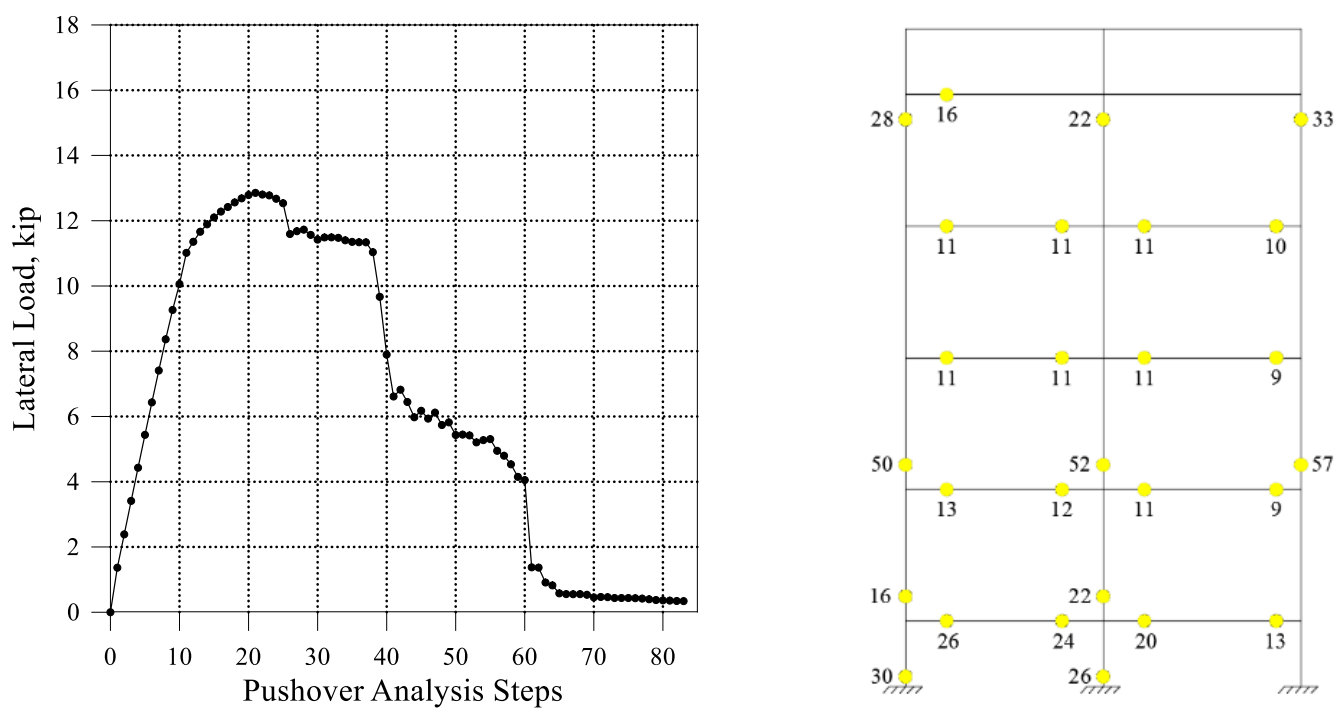

Figure 6.11 Fiber plastic hinges reinforcement yield progress mechanism for G-FB wall. 


\subsubsection{Influence of EPS blocks}

To measure the influence of EPS blocks on the overall performance of the SGICF walls, two numerical models have been developed to perform pushover analysis. The first numerical model was of W-RC wall after eliminating the EPS infill struts. The second numerical model was of G-RC wall after adding the EPS infill struts. For this purpose, an assumption was made to use the same properties of EPS blocks that were used for W-RC wall with consideration of G-RC wall dimensions to calculate the infill struts as shown in Figure 6.12.

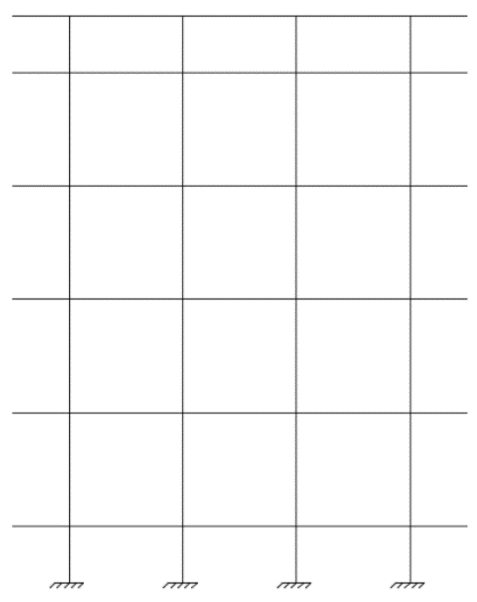

(a)

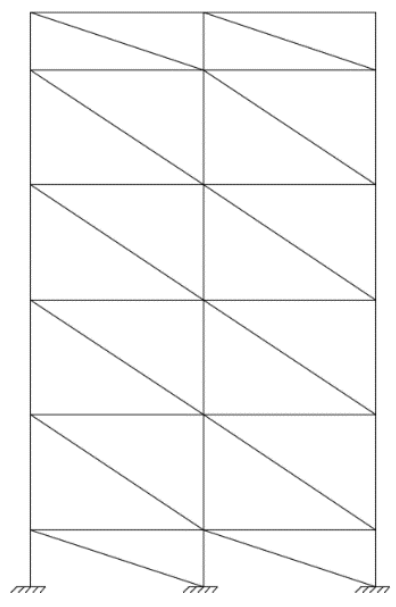

(b)

Figure 6.12 Numerical models: (a) W-RC without EPS struts, (b) G-RC with EPS struts.

Figure 6.13 shows the pushover analysis results for both W-RC and G-RC specimens. The EPS struts influence on the maximum lateral strength of the W-RC wall was very small and the maximum lateral strength was $99 \%$ of the model that has the EPS 
struts. At lateral displacement of 2in., the influence of the EPS struts was notiable and the lateral strength of the model that has no EPS struts was $59 \%$ of the model that has the EPS struts. For G-RC model, the maximum lateral strength was $96 \%$ of the model that has the EPS struts. At lateral displacement of 2in., the influence of the EPS struts was also notiable and the lateral strength of the model that has no EPS struts was $75 \%$ of the model that has the EPS struts. It was shown that the engagement of the EPS struts did not start until the lateral displacement increased, which was an expected behavior due to the fact that the EPS material required high strain to reach the maximum resistance stress as was shown in Figure 6.3.

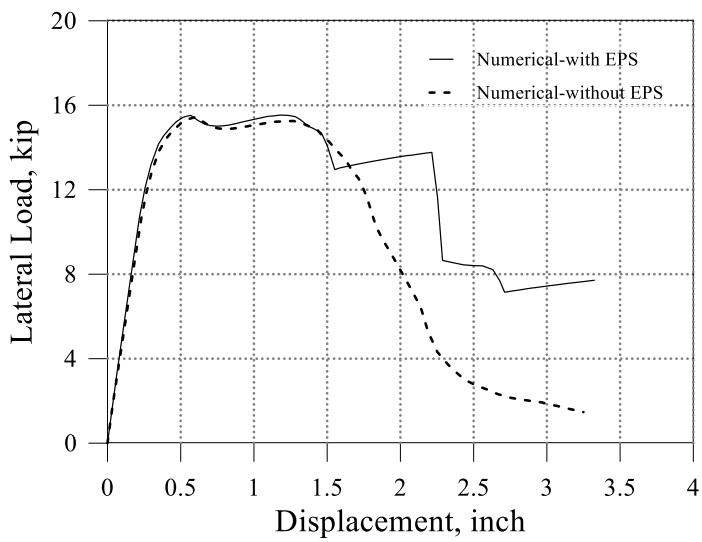

(a) W-RC

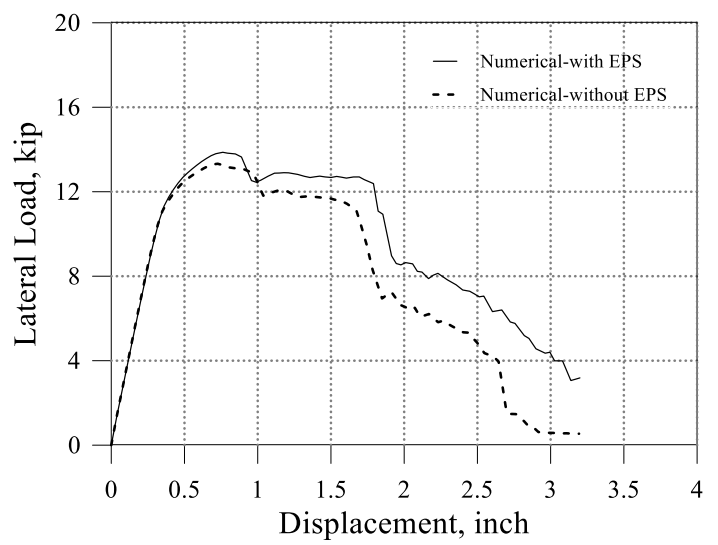

(b) G-RC

Figure 6.13 Comparison of numerical models results for W-RC and G-RC specimens. 


\subsection{Summary of the Numerical Analysis}

Numerical analysis was considered to evaluate the initial stiffness and the lateral strength of the SGICF walls with a comparison to the experimental results. Evaluation of the EPS blocks influence on the lateral strength was also considered for both types of the SGICF walls. A quasi-static nonlinear analysis with fiber-based hinges was adopted in the numerical modeling. The numerical results showed that the models could provide a reasonable approximation of the nonlinear behavior of the SGICF walls, including the stiffness and the maximum lateral strength. The peak loads provided by the numerical models were close to the experimental results. The overall nonlinear behavior and the shape of the pushover curves were in good correlation with the experimental data. Also, the numerical models showed that the influence of the EPS blocks on the maximum lateral strength was very small, while the effect of the EPS blocks started to increase when the lateral drift increased. 


\section{CHAPTER 7: Conclusions and Recommendations}

The primary purpose of the experimental work in this study was to investigate the dynamic properties and the in-plane lateral strength of the full-scale SGICF walls. Shaking table tests were conducted on two sets of SGICF walls. The first set was made from dry stacking blocks of recycled expanded polystyrene forms and reinforced concrete

cores, while the second set was designed and built using a different technique. Each set of the SGICF walls was constructed with nominally identical geometry including the overall aspect ratio as well as the same size, spacing, and rebar detailing for the cores. Steel fiber-reinforced concrete was used in one of the walls of each set in an effort to increase the shear strength of the individual cores and enhance the overall wall drift capacity.

Further, this study included another experimental investigation at the component level that was conducted on six small-scale circular reinforced concrete beams to investigate the influence of steel fiber-reinforced concrete on the shear capacity of the individual core of the two types of SGICF walls under cyclic loading. In addition to the experimental programs, analytical and numerical methods were discussed and employed to evaluate the lateral strength and the initial stiffness of these walls. The first section of this chapter presents the main conclusions from this study. The second section provides recommendations and suggestions for future studies to further improve the knowledge on the dynamic behavior of the SGICF walls. 


\subsection{Conclusions}

Several conclusions were drawn based on the experimental results obtained from the shake table experiments of the full-scale SGICF walls, quasi-static experiments at the component level, analytical methods, and numerical analysis:

1. SGICF walls that were built from EPS blocks showed stiffer behavior when compared to the newly suggested pattern of SGICF walls. Both W-RC and W-FB walls had a natural frequency period that was less than that obtained for G-RC and GFB walls. The extra vertical core in the W-RC and W-FB walls contributed to the higher initial stiffness.

2. The failure modes of the $\mathrm{W}-\mathrm{RC}$ wall were caused by rebar fracture at the base, while the wall with the steel fiber-reinforced cores (W-FB) gradually degraded. Both walls reached similar levels of drift capacity, and the wall with the steel fiber-reinforced cores exhibited $77 \%$ of the strength of the wall without fibers. These differences can be mainly attributed to the voids in the cores of the fiber reinforced concrete cores that were observed after stripping away the polystyrene stay-in-place forms. This result highlights the importance of workability and potential unsuitability of steel fiber-reinforced concrete used within the small core sizes that exist in SGICF walls given that the walls were cast at the same time, used the same placement techniques and utilized concrete mixes of a similar slump.

3. The drift ratio that was measured in the south direction exceeded $2.5 \%$ for both $\mathrm{W}$ RC and W-FB walls, which is a common drift limit in most structural design codes. 
This was not the case for the drift ratio that was obtained from the north direction, which was less than $1.5 \%$ for both walls. The variation in the drift ratios could be attributed to the fact that the inertia masses were pulling the walls towards their sides after each applied scaled ground motion, especially when the walls started to degrade and lose their integrity. In addition, the concentration of the voids found in the W-FB wall was in the south direction that contributed to the asymmetric behavior.

4. Placing two rebars in the vertical cores of the newly suggested pattern of SGICF walls and increasing the spacing between the vertical cores influenced the failure modes and upgraded the lateral drift ratios of both walls. Stiffness degradation was dominant for the G-RC wall while crushing of the concrete caused the collapse of the G-FB wall. The symmetric deformation was observed for G-RC wall at the end of the ground motion scaled to 1.25 , with a maximum drift ratio of $3.8 \%$. Unlike the drift ratios of the G-FB wall for the same level of ground motion, which were $3.47 \%$ in the south direction and $1.85 \%$ in the north. The concrete honeycomb found on some of the horizontal cores of the G-FB wall could contribute to the asymmetric drift deformations.

5. The use of the steel fiber-reinforced concrete in the G-FB walls did not improve the lateral resistance when compared to the G-RC wall, but the influence of the steel fiber-reinforced concrete was apparent on the lateral drift capacity. The maximum drift ratio was $4.9 \%$ for the G-FB wall at the end of the ground motion scaled to 1.5 , which was higher than the drift limit reached by other tested walls. 
6. The quasi-static tests conducted on the component level beams provided a similar strength for both test setups. The influence of the steel fiber-reinforced concrete did not improve the strength capacities of these specimens. Furthermore, there was not an apparent difference in the deflection capacities between specimens that had reinforced concrete and the one that had steel fiber-reinforced concrete. This could be related to the fact that the Helix steel fiber dose played a primary role in affecting the expected improvement in the specimen's strength and failure modes. Since the method that was used to calculate the required Helix steel fiber specified a different dose based on the structure type and usage, using the same dose that was used for the SGICF walls did not provide the anticipated improvements in the tested beams. Moreover, fiber length and the bond characteristics between the fibers and the concrete could be a reason for the lack of improvements.

7. The presented analytical methods did not provide a close estimation of the initial stiffness of the SGICF walls. The ESM method was the only approach that provided a close estimation of the initial stiffness for all SGICF walls when compared to the other methods with an average overestimation ratio of $180 \%$.

8. Two methods were able to estimate the lateral strength of the SGICF walls. The results obtained from using the ACI equation for the minimum shear strength of the concrete $\left(\mathrm{V}_{\mathrm{c}}\right)$ were accurate in estimating the lateral strength of the three walls. The W-FB wall was the only wall that this method overestimated its lateral strength by $22 \%$, and this can be justified by the voids found in the W-FB wall, which influenced its lateral strength. The $\sum \mathrm{V}_{\mathrm{cm}}$ method was the second method that managed to provide 
a good estimation for the lateral strength of the SGICF walls with an average overestimation ratio of $103 \%$ for all walls.

9. Numerical models were developed in SAP2000 in order to characterize the nonlinear response of the SGICF walls. The numerical results showed that the models could provide a reasonable approximation of the nonlinear behavior of the SGICF walls, including the stiffness and the maximum lateral strength. The peak loads provided by the numerical models were close to the experimental results. The overall nonlinear behavior and the shape of the pushover curves were in good correlation with the experimental data.

\subsection{Recommendations}

The evaluation of the dynamic response of the SGICF walls with circular crosssections under self-weight loads by using shake table experimental tests was the primary outcome of this study. Future research is required to test different SGICF walls types with various aspect ratios and loading conditions, and it would be beneficial to widen the use of these walls in areas that are prone to seismic activities. Recommendations for future works are summarized below:

1. The SGICF walls that were tested in this investigation have one aspect ratio. Conducting shake table tests on SGICF walls with different aspect ratios is needed to provide a good understanding of the dynamic behavior of these walls during seismic events. 
2. In this study, one steel rebar was used in each vertical core of the SGICF walls that were built out of EPS blocks. The use of two steel rebars in each vertical core is recommended to measure the improvements in the drift capacity.

3. Using different types of fibers that provide acceptable workability within the small core sizes that exist in SGICF walls is needed to overcome the lack of concrete consolidation.

4. Many buildings were built with different types of ICF blocks that have different cores shapes. Shake table experiments are recommended on ICF walls with rectangular cores cross-sections.

5. Conducting shake table tests on SGICF walls with doors and windows opening is recommended to measure the influence of these opening on the overall dynamic behavior.

6. Adding a constant axial load while conducting shake table tests on the same SGICF walls to widen the knowledge of the possibility of using SGICF walls in multi-story buildings.

7. Construct an equivalent flat ICF wall that has the same concrete volume and reinforcement of the SGICF wall to evaluate the economic influence and measure the lateral strength. 


\section{References}

Abdel-Mooty, M., Haroun, M., El-Maghraby, Y., Fahmy, E., \& Abou-Zeid, M. (2010). Performance of screen grid insulating concrete form walls under combined inplane vertical and lateral loads. Advanced Materials Research, 163-167, 18031810.

ACI 318-14. (2014). Building code requirements for structural concrete (ACI 318-14) and commentary (ACI 318R-14). Farmington Hills, MI: American Concrete Institute.

American Concrete Institute (ACI). (2008b). Acceptance criteria for special unbonded post-tensioned precast structural walls based on validation testing and commentary. ACI ITG-5.1-07, Skokie,IL.

American Concrete Institute. (2001). Acceptance criteria for moment frames based on structural testing. ACI T1.101, Farmington Hills, MI.

American Society of Civil Engineering (ASCE). (2000). Prestandard and commentary for the seismic rehabilitation of buildings. Washington,DC: (FEMA-356). Federal Emergency Management Agency.

Aydemir, C., Zorbozan, M., \& Alacali, N. S. (2009). Determination of moment capacity MP for rectangular R/C columns. Tech. J. Turk. Chamber Civ. Eng., 20(1), 45454565 .

Carrillo, J., Gonzalez, G., \& Llano, L. (2012). Evaluation of mass-rig systems for shaking table experiments. Dyna, 79(176), 159-167. 
Chang, Z.-T. (2014). Report on investigation of bending and shear behaviors of helix circular piles. Sydney: The University of New South Wales.

Chopra, A. (2011). Dynamic of Structures. Theory and Applications to Earthquake Engineering.Fourth edition. Prentice Hall.

Computer \& Structures INC. (2016). Parametric P-M2-M3 hinge model. Technical note. Computer \& Structures INC. (2011). CSI Analysis Reference Manual For SAP2000®, ETABS ${ }^{\circledR}, S A F E ®$ and $C$ SiBridge ${ }^{\mathrm{TM}}$.

Computers and Structures Inc., (CSI). (2017). SAP2000, structural analysis program. Version 19.2.1. Berkeley, California, USA.

Cook, R. D., Malkus, D. S., Plesha, M. E., \& Witt, R. J. (2001). Concepts and Applications of Finite Element Analysis (4th ed.).

Dusicka, P., \& Kay, T. (2011). In-plane lateral cyclic behavior of insulated concrete form grid walls. Journal of Structural Engineering, 1075-1084.

Dusicka, P., \& Werner, C. (2012). Potential strategies for improving cyclic performance of screen grid insulated concrete form walls. 15th World Conference for Earthquake Engineering. Lisbon, Portugal.

Garth, J. (2014). Experimental investigation of lateral cyclic behavior of wood-based screen-grid insulated concrete form walls. Portland State University. doi:10.15760/etd.1856

Habibullah, A., \& Pyle, S. (1998). Practical three dimensional nonlinear static pushover analysis. Structure Magazine. 
Hsiao, J. K. (2014, November). Hand-calculated procedure for rigidity computation of shear walls with openings. Practice Periodical on Structural Design and Construction, 19(4), 04014017 (12 pp.).

ICC Evaluation Service. (2012). APEX Block Interlocking System. ICC-ES Evaluation Report ESR-1770.

Mehrabi, A. (2000). In-plane Lateral Load Resistance of Wall Panels in Residential Buildings. Skokie,IL: Portland Cement Association.

NAHB Research Center,Inc. (1998). Structural Design of Insulating Concrete Form Walls in Residential Construction. Upper Marlboro, MD.-PCA R\&D Serial No. 2164.

NAHB Research Center,Inc. (2001). In-Plane Shear Resistance of Insulating Concrete Form Walls. Upper Marlboro,MD.

Ochieng, E., Wynn, T., Zuofa, T., Ruan, X., Price, A., \& Okafor, C. (2014). Integration of Sustainability Principles into Construction Project Delivery. Architectural Engineering Technology, 3(1).

Polytrox,LLC d.b.a Helix Steel. (2016). Helix 5-25 micro-rebar concrete reinforcerment system. Ann Arbor: Helix steel. Retrieved from http://www.helixsteel.com/technical

Sultan, A. (2016). Navigation the untapped potential of insulated concrete forms as longterm urban refugee housing in the middle east. International journal of science and technology, 5(10), 523-536. 
Tena Colunga, A., \& Rivera Hernández, G. (2015). Assessment of the lateral stiffness of walls with openings. COMPDYN 2015. Crete Island, Greece: 5th International Conference on Computational Methods in Structural Dynamics and Earthquake Engineering.

Werner, C. (2010). Cyclic behavior of screen grid insulated concrete form components. Portland State University. doi:10.15760/etd.1856

Yang, L., Li, L., \& Zhu, H. (2012). The simplification calculation method of the beamcolumn joint rotation of frame structures under static load. ISSE 2012 : Proceedings of the 12th International Symposium on Structural Engineering. Wuhan, China: Science Press.

Yland, C. (2000). Experimental and Analytical Studies of the Structural Performance of Innovative Structural Grid Walls. Irvine, California: University of California Irvine. 